\title{
MEASURING COMMUNITY RESILIENCE TO DISASTER
}

\author{
A Thesis \\ presented to
}

the Faculty of the Graduate School

at the University of Missouri-Columbia

In Partial Fulfillment

of the Requirements for the Degree

Master of Arts

by

AARON MATTHEW WESLEY

Dr. Timothy Matisziw, Thesis Supervisor

May 2014 
The undersigned, appointed by the dean of the Graduate School,

\author{
have examined the Thesis entitled \\ MEASURING COMMUNITY RESILIENCE TO DISASTER \\ Presented by Aaron Wesley \\ A candidate for the degree of \\ Master of Arts
}

And hereby certify that, in their opinion, it is worthy of acceptance.

[DR. TIMOTHY MATISZIW]

[DR. MARK PALMER]

[DR. PRAVEEN EDARA] 


\section{ACKNOWLEDGEMENTS}

I would like to thank the following employees of the City of St. Louis for their high interest in and assistance collecting data for this project: David Sychoras, Anneliese Stover and Sarah Figueroa of St. Louis Area Agency on Aging; Matthew Mourning of St. Louis Urban Planning and Design Agency; Dottie Pennington of the St. Louis Citizen's Service Bureau; and Leah Freeman of the St. Louis Metropolitan Police Department. Additionally, I am very grateful to Jennifer Reiman of East-West Gateway Council of Governments and Nancy Thompson of St. Louis Association of Community Organizations for arranging access to datasets on St. Louis neighborhood characteristics.

I owe much thanks to the faculty of the Department of Geography for their exceptional academic support these last two years. Special thanks goes to Dr. Timothy Matisziw, who provided the intellectual challenges behind many of the new methodologies developed in this thesis (and put up with my lack of proofreading skills). For their prompt attention and helpful comments, I thank Dr. Mark Palmer and Dr. Praveen Edara.

A huge thank you goes to all my friends and family whose support made it possible for me to complete my degree, especially to my dad Dana Wesley who helped me out innumerable times.

Last and most importantly, thank you Lindsay for your love and patience - you were always my motivation, from start to finish. 


\section{TABLE OF CONTENTS}

ACKNOWLEDGEMENTS ii

LIST OF FIGURES Vi

LIST OF TABLES

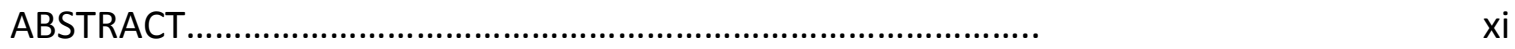

$\begin{array}{lll}\text { Chapter Page } & \text { Pag } \\ & & \end{array}$

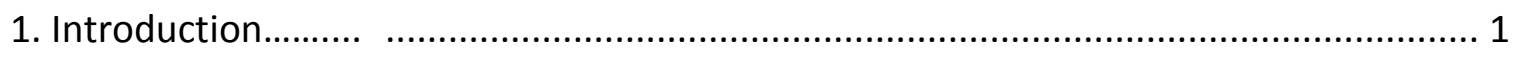

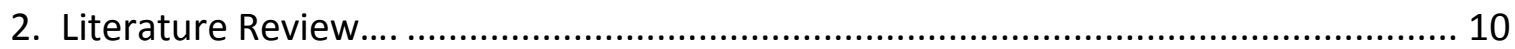

2.1 Hazards, Disasters and Vulnerability in

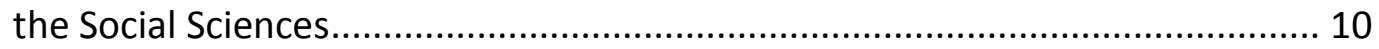

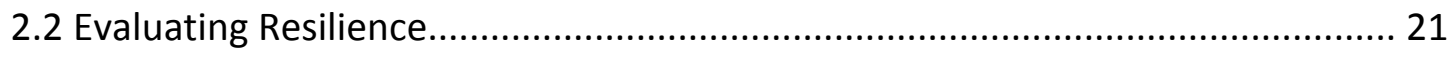

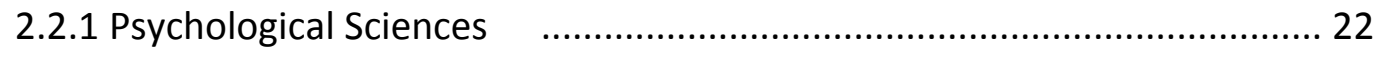

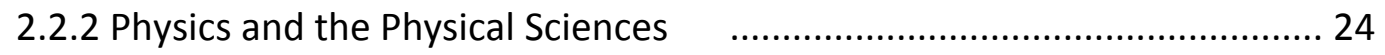

2.2.3 Ecology and Social-Ecological

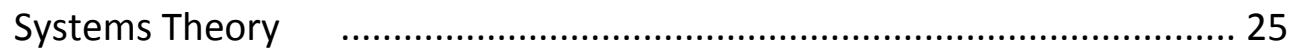

2.2.4 The Shift of Focus From Community

Vulnerability to Community Resilience $\quad$............................................. 33

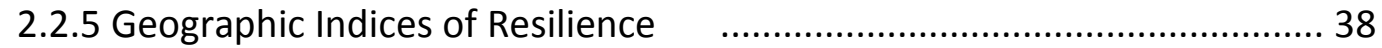

2.2.6 Accounting For Uncertainty in Measurement of Community Resilience .......................................................................... 43

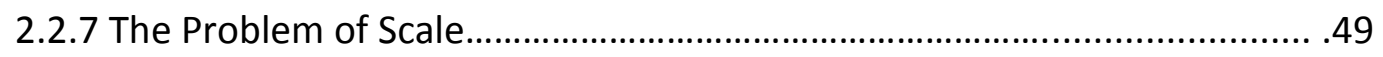

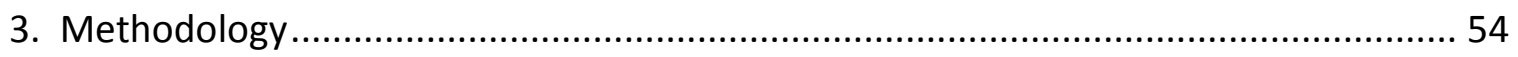

3.1 Environmental Hazard Profile................................................................... .55 
3.2 Combined Environmental and

Socioeconomic Vulnerability Index

3.3 Resilience Index

3.4 Determining Indicator Weights .

3.5 Combining Vulnerability and Resilience Indices.

3.6 Validation Methodology. 64

3.7 Evaluating Indicator Inter-Influence, Identifying Cause-Effect Relationships and Recommending Indicators For Targeted Mitigation Strategies

4. Application to St. Louis, Missouri

4.1 Study Area and Units of Analysis

.72

4.2 Data Sources .74

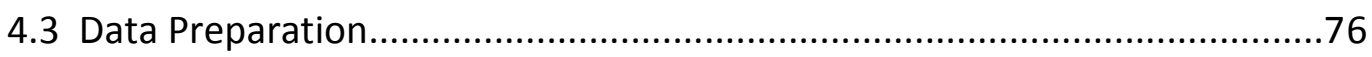

4.4 Vulnerability Indicators and Index................................................. . 77

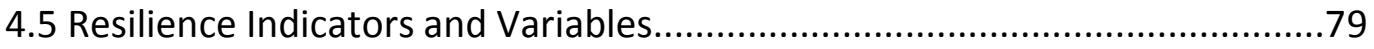

4.6 Validation Datasets 83

5. Results

5.1 Hazard Profile, Vulnerability Index and Sub-Index Weights

5.2 Absolute Resilience Index and Sub-Index Weights

5.3 Confusion Matrix and Relative Resilience Classifications

5.4 DEMATEL Results and Indicator Influence Diagram

6. Validation And Discussion 
6.1 Internal Validation: Model Sensitivity to

Sub-Index Weighting Scheme

6.2 External Validation: Model Correlation to

Observed Disaster Resilience

99

6.2.1 Case \#1: Extreme Heat-Related Fatalities

6.2.2 Case \#2: Population Loss Due to Economic Shocks

102

6.2.3 Case \#3. Resident Requests For Government Assistance After Windstorms

6.3 Model Correction For Validation Results

6.4 Spatial Patterns of High and Low Resilience in St. Louis

6.5 Autocorrelation and Spatial Influence of

Neighborhood Resilience

6.6 The Case For Resilience Model Validation and Correction

6.7 Which Neighborhoods Should Emergency Managers

Focus On?

6.8 What Community Characteristics Should Emergency

Managers Target?

6.9 Model Modifications, Alternative Methodologies and

Future Directions For Community Resilience Research

7. Conclusion

APPENDIX

A. ENVIRONMENTAL HAZARD VARIABLES

B. INDICATORS OF VULNERABILITY

C. INDICATORS OF RESILIENCE 150

BIBLIOGRAPHY 


\section{LIST OF FIGURES}

Figure

1. The Adaptive Renewal Cycle in Socio-Ecological Systems Theory ......................... 28

2. Nested Multi-Level Renewal Cycles: The Panarchy Concept .................................. 29

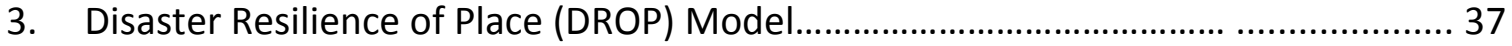

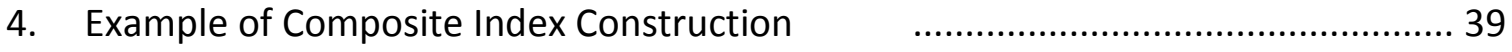

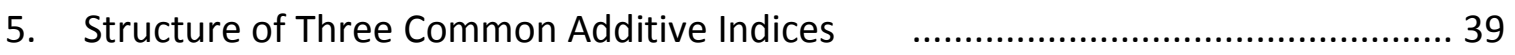

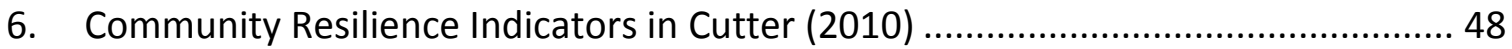

7. Example of a Large-Scale Community Resilience Study....................................... 50

8. Sample Vulnerability and Resilience Index Score Distributions and Statistics ......... 63

9. Sensitivity Analysis Comparison of 3 Common Additive Indices .............................65

10. Example Casual Diagram Output from a DEMATEL Analysis .................................. 70

11. Example Network Diagram Output from a DEMATEL Analysis ............................. 71

12. Study Area and Analysis Units- St. Louis City Neighborhoods ............................. 73

13. Distribution and Standard Deviation Categories of Vulnerability Index ................... 84

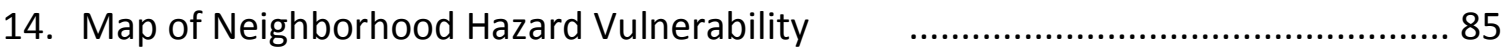

15. Distribution and Standard Deviation Categories of Resilience Index ..................... 86

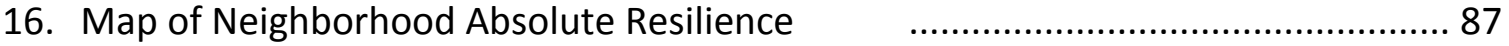

17. Distribution of Neighborhoods in Relative Resilience Categories ......................... 88

18. Map of Neighborhood Relative Resilience (Pre-Validation Classification) ............... 89 
19. Causal Diagram of Resilience Indicators Evaluated in DEMATEL Analysis

20. Example Fuzzy Representation of Neighborhoods

With Uncertain Relative Resilience Classifications

21. Resilience Model Validation With Heat Death Data

22. Abrupt Population Loss in St. Louis Following 2008 Financial Crisis

23. Resilience Model Validation With Post-Economic Shock Population Change 103

24. Resilience Model Validation With Storm-Related Requests For Assistance. 106

25. Reclassification of Original Relative Resilience Categories 108

26. Reclassified Relative Resilience Categories with Fewer 'Medium' Neighborhood Categorizations

27. Significant Clustering of High and Low Relative Resilience Neighborhoods

28. Distribution and Standard Deviation-Based Categories of Incoming Resilience Influence $T_{\mathrm{IR}}$

29. Changes to Relative Resilience Categories after Spatial Influence Analysis

30. Map and Population Tables of Relative Resilience Categories after Spatial Influence Analysis 120

31. Correlation of Heat Death Data with Corrected Resilience Model

32. Correlation of Windstorm-Related Requests for Assistance with Corrected Resilience Model

33. Comparison of the Present Study's Relative

Resilience Classification and the Alternate Classification.

34. Comparison of Statistically-Significant Clustering of Resilience Classifications Between the Relative Resilience Model and Alternate Model....126

35. Coincidence of Heat Deaths and Resilience Classifications Between Relative Resilience Model and Alternate Model .128 
36. Coincidence of Requests for Assistance and Resilience Classifications

Between Relative Resilience Model and Alternate Model..

37. Example 3D representation of St. Louis Neighborhood

Population and Resilience Categorization

viii 


\section{LIST OF TABLES}

Table

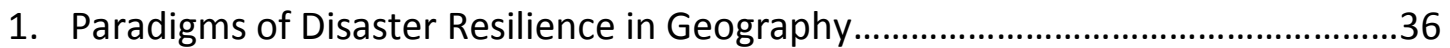

2. Stages of Composite Index Construction ..................................................................

3. Quantitative Scale for Indicator Comparisons...................................................60

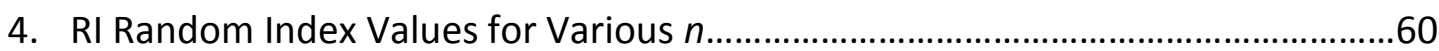

5. Sample Confusion Matrix to Convert Vulnerability and Resilience Categories to Final Relative Risk Categories.......................................................63

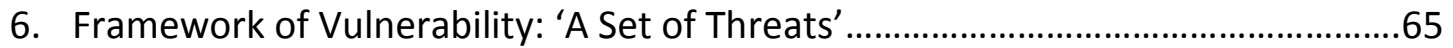

7. Digraph Structure for AHP Assessment of Socio-economic Vulnerability Sub-Index.

8. Framework of Resilience: 'A Set of Protections' .79

9. Digraph Structures for AHP Assessment of Resilience Sub-Indices........................80

10. Digraph Structure for DEMATEL Analysis of Resilience Indicators .81

11. Results of Environmental Hazard Assessment from City of St. Louis Mitigation Plan

12. Results of AHP Assessment of Socio-Economic Vulnerability Indicators. .84

13. Results of AHP Assessment of Resilience Index Sub-Levels..................................86

14. Neighborhood Classified by Vulnerability/Resilience ............................................8

15. DEMATEL Matrix N (Normalized Direct Relation Matrix) .......................................90

16. DEMATEL Matrix T (Total Direct-Indirect Matrix) ..................................................90

17. Degree of Dispatching Pi, Positional and Relational Values for Resilience Indicators Used in the DEMATEL Analysis. 
18. Baseline and Alternative Weighting Schemes Used In Sensitivity Analysis.

19. Changes to High-Risk Neighborhood Categorizations

Due to Alternative Weighting Schemes...............................................................95

20. Spatial Weights Matrix Computed for Neighborhoods \#1-5................................114

21. Normalized Distance/Population Weights WN

Calculated for Selected Neighborhoods..................................................................116

22. Directed Resilience Influences $R_{\mathrm{l-j}}$ Calculated

for Selected Neighborhoods. 


\title{
Measuring Community Resilience to Disaster \\ Aaron Wesley \\ Dr. Timothy Matisziw, Thesis Supervisor
}

\begin{abstract}
Although geographic studies of disaster vulnerability and resilience have been central to the formulation of federal emergency management policy, recent community resilience research has diverged significantly from the core foci of the discipline: the importance of place, of scale, and the complexity of human-environment interactions. Three disconcerting trends in the literature can be observed. First, there has been a heavy reliance on the tools of linear systems science to characterize and measure the human dimensions of resilience - dimensions which are increasingly examined in terms of their nonlinearity, dynamism and complexity in other scientific disciplines. Second, most of the variables typically used as proxies for community resilience are not actually indicative of community-scale processes, but rather describe individual-scale behavioral and household-scale socioeconomic characteristics. Third, the current practice of aggregating resilience indicators to large, heterogeneous geographic areas in order to communicate community-level resilience can actually mask and mischaracterize the local, place-specific variability of those indicators. This thesis presents a rethinking of geography's conceptual model of population disaster resilience and the methods used
\end{abstract}


to measure it at the community level. Drawing on diverse theoretical linkages on the subject from across the social and natural sciences, and on the current perspectives and information requirements of local emergency managers, a more holistic and meaningful approach to measuring community resilience is proposed. Specifically, in recognition of a need to integrate both expert and lay local perspectives into resilience calculations, a system for assimilating such qualitative data into quantitative analysis is adapted from complexity theory. Also, in acknowledgement of the multiple levels at which resiliencebuilding processes may operate in human systems, and the unique ways disaster resilience can manifest in different places, a new framework for balancing multi-scalar indicators of community resilience for local-level analysis is proposed. As a proof-ofconcept for the above approach, a community disaster resilience analysis is conducted at the neighborhood level in the City of St. Louis, Missouri. Results indicate that analyses which factor in local knowledge of both hazards and unique protections against those hazards can explain observed community-level resilience to actual disasters better than analyses which rely solely on internal statistical techniques. Results also show that the proposed method for analysis of qualitative expert assessments of local resilience indicators can unveil the complex structure of cause-effect influence among these variables and can reveal which community assets are most important to nurturing disaster resilience. 


\section{CHAPTER 1. INTRODUCTION}

Most people know enough about environmental hazards to want to protect the things they value against hazards' effects. But when faced with a variety of threats both natural and social in origin, most people are highly uncertain about which protections are necessary in order to be ready to face an extreme environmental event. And they do not know how the two - protection and readiness - interact. One consequence of this is that most disaster preparedness insights, and the clearest assessment of a community's deficiencies in protection and readiness, are often observable only after disaster strikes - even though much a priori knowledge about the number and nature of hazards exists. There is much academic interest in making sense of the hazards to which populations are vulnerable and identifying the various protections which foster community resilience to their effects - and for good reason. In light of the hazardmagnifying effects of global climate change (IPCC 2013), and the increasing concentration of populations and infrastructure in large urban centers within hazardprone areas (NRC 2012), a high priority must be placed on research which reduces uncertainty about the processes and structures which lead to disaster resilience. The research presented here attempts to accomplish this by critically examining theoretical and methodological issues in the study of community resilience, with the goal of providing a more holistic perspective and insight to disaster management policy.

In the context of most geographical research, a natural hazard refers to physical environmental threats to people and places arising from the intersection of human and 
natural systems (Cutter 2003; Cutter et al. 2003). Vulnerability refers to a certain place's potential for harm from natural hazards, as a function of exposure (spatial cooccurrence), risk (frequency of occurrence), and severity (relative magnitude). Hazard vulnerability, then, is defined in this thesis as the degree to which a particular location interacts with environmental threats in time and space. There are several advantages to this conceptualization. First, this definition is applicable across a range of spatial scales, from cells to individual organisms to entire ecosystems. Second, when defined as such, hazard vulnerability exists everywhere given that threats to survival are present everywhere to some extent (Paul 2011). All organisms and communities of organisms live with some degree of vulnerability, and then make choices, take risks, develop defenses, etc. to mitigate against environmental threats. Third, vulnerability in this sense is a manageable concept to measure, involving the enumeration of the hazards a location is exposed to, as well as the spatiotemporal characteristics of those hazards that make them more or less of a threat. Calculations of vulnerability could be as simple as a binary checklist of environmental hazards in a local ecosystem; conversely, they could combine detailed quantification of exposure, extent, frequency and severity into a complex algorithm. Perhaps the most important advantage to this conceptualization of vulnerability is that it is segregated from the more complex concept of disaster resilience.

In this thesis, natural disasters are defined as extreme environmental events, arising from natural hazards, which overwhelm a system's capacity to absorb, respond to or recover from damage - often leading to a shift into a less productive or otherwise 
unfavorable system state. Resilience is considered here to be a scalable organic concept referring to all the defenses, resources, adjustments, capacities, connections and attributes which organisms or communities of organisms 'put into play' to plan for and mitigate against environmental hazards, absorb the effects of extreme events, and prevent lapse into an unfavorable state. Resilience in this sense is a quality that appears at all levels of nested organic systems from individual cells (and even intracellular components) to individuals, communities and higher-order networks of communities. This definition of resilience highlights the vast integrative potential for disaster research; whether or not it is explicitly referenced as 'resilience', the phenomenon described above has been considered in business, biology, statistical physics, developmental psychology, computer science, and many other disciplines.

However, along with all the interest in resilience comes the caveat that its components are not well understood or even defined, and that significant disagreement and uncertainty exist in how to measure resilience or what a resilient system looks like in the real world. As later reviewed, emerging in the literature are common themes of various capacities (e.g., adaptive, learning, absorptive, resistive, coping, etc.) as well as a sense of a need for structured self-organization and strategic connectedness. Yet, these qualities seem to emerge and manifest themselves in very different and even surprising ways in different places, an observation which has undoubtedly contributed to geographers' attraction to the subject.

In light of the above, the task of defining the term community should be approached with a healthy sense of caution. Is it better to include the descriptor 
'geographically bounded' in the definition, since this conforms well to dominant conceptualizations of space within geography and the modalities of its cartographic discourse (Norris et al. 2007)? Or, should the unbounded and open nature of communities in today's hyper-connected, globalized world be stressed (Wilson 2012)? Would a homogeneity-oriented characterization of a group of people united by shared interests, collective experience and common goals be charitable (Kulig 2000)? Or would this unfairly preclude the existence of competing networks of power, actors and interest groups with divergent aims, or a complex heterogeneity of sometimes incompatible worldviews (Allen 2003)? Researchers who choose a certain definition of community over another are both right and wrong for reasons rarely acknowledged: while all researchers operationalize 'community' according to their own conceptual orientation and in accordance with their studies' research goals, the fundamentally place-specific nature of how communities manifest necessitates reevaluation and reinterpretation of the definition for different place contexts and assures that no single definitions will be universally applicable or acceptable. ${ }^{1}$ With that caveat, the definition of community used in this thesis - a population living within a self-delineated or historically distinguishable local area who can reasonably be expected to come together to solve problems - is less of a normative statement and more of an invitation for the reader to explore the place-based rationale for this choice.

\footnotetext{
${ }^{1}$ A significant portion of this thesis explores how the nascent discipline of complexity science deals with concepts that resist discrete, unambiguous or authoritative definition, or that emerge differently at small scales or in different parts of a system.
} 
There exists a long and rich history of research spanning many disciplines which has informed the emergency management policy community about the characteristics of natural hazards and the populations exposed to them. The pervasiveness, complexity and scale-invariance of human system interaction with extreme environmental events has fascinated and challenged an impressively diverse set of disciplines. For instance, psychologists have studied disaster-induced psychological trauma in individuals (Kutak 1938), sociologists have sought to explain a farm family's decision to settle on floodplains (Chan 1995), geographers have analyzed differences in community-level evacuation route accessibility in a city (Cova and Church 1997), economists have modeled the regional effects of a volcanic eruption shutting down the world's busiest airport (Budd et al. 2011), and climatologists have attempted to predict climate change's impact on global storm intensity zones (IPCC 2013).

The interplay of natural and social systems that give rise to disasters have long attracted the attention of academic geography in particular, which has always strove, with varying degrees of success, to stake claim to studying phenomena that bridge the social and natural sciences (not to mention that geographers are irresistibly drawn to processes which demand considerable attention to scale). In fact, it was geographer Gilbert White who is credited with establishing the academic credibility of and stimulating widespread interest in hazards and disasters research in the years following WWII (Quarentelli and Dynes 1977; Smith 2001). While the story of how the subject has since attenuated into the work of other disciplines is certainly complex, it is fair to state 
that research which attempts to make sense of hazards and extreme events is by now ubiquitous across academia.

Yet, for all the interdisciplinary relevance the subject has found, a common critique which arises in the current review literature is that hazards research could benefit from more cross-disciplinary collaboration (Vogel et al. 2007; Miller et al. 2008;

Turner et al. 2010; Berkes and Ross 2013; Downes et al. 2013). It is observed that the study of disaster commonly proceeds in a compartmentalized fashion, with researchers in each field tending to adhere to in-house theories and methods, many of which can claim decades-long traditions of scholarly development (Taylor 1984; Carrerra and Guzzetti 1993; Alexander 1997; Teo et al. 2013). While many researchers recognize both the inevitability of a certain amount of stovepiping in academia as well as the benefits of theoretical maturation this process provides, it is contended that, at the very least, there exists a strong, largely unrealized potential for trans-disciplinary collaboration which includes cross-adaptation of theories and methods.

The normative argument that the above should be happening - that very substantive progress in the subject could be enabled by an actively integrative element - is, at least on paper, readily apparent within geography. On the one hand, those involved in hazards and disaster research are apt to include appeals for future research to be more integrative and inclusive of other professional viewpoints. On the other hand, the current dominant paradigm of disaster research in geography specifically codifies transdisciplinary linkages, as well as methodological pluralism (the willingness to integrate multiple research methods and technologies) and multi-perspective 
reflexivity (the willingness to learn from a variety of knowledge sources, both lay and expert), as norms by which research should proceed (Cutter 2003, Miller et al. 2008). Given the wide credence and citation these three foci currently enjoy, it would be an ironic and understandably contentious assertion that the recent thread of community disaster resilience research in geography has not successfully lived up to these norms. Such an assertion is made in the present thesis.

The argument presented is threefold. First, it is asserted that methodologically, the continued approach of aggregating and analyzing resilience variables with overlysimplistic indices is in direct conflict with current multidisciplinary understandings of the complex and interrelated nature of the components of resilience. To account for these dynamics, a methodology from the field of industrial risk management can be adapted for identifying the structure of complex cause-effect relationships inherent in index variables, using a knowledge-based system. Second, it is argued that the majority of variables currently used in geographic studies of community resilience are not holistically indicative of community-level processes, but rather disproportionately describe individual-level behavioral and household-level socioeconomic characteristics. As such, a more multi-scalar approach to variable selection is proposed which captures and balances individual- as well as community- and government-level processes and characteristics, chosen from place-specific research of the community in question. Third, based on multidisciplinary understandings of the fundamentally endogenous, place-based nature of resilience, and taking into account the place-specific information requirements of emergency managers, a paradigmatic shift away from the current 
practice of aggregating resilience variables over large geographic areas in favor of localscale geographic analysis based upon a more realistic construct of a community is proposed.

To illustrate the benefits of the developed approach, an analysis of community resilience to disasters within the City of St. Louis, MO is conducted, utilizing indicators of community resilience that not only represent the multiple facets of resilience but also the multiple scales at which resilient characteristics and processes operate. Rather than relying solely on internal multivariate statistics to evaluate or correct inter-influence of study variables (a method which frames such influences as design errors, possibly leading current studies to eliminate important explanatory variables), the proposed approach seeks to account for and explore variable influence, including the structure of complex cause-effect relations, using a knowledge-based rating system. Further, methods from within complexity science are extended to extract perceived comparative variable influence on the overall management goal of increased disaster resilience.

The aim of this research is not only to help bring the study of community resilience in geography up to speed with its peers by bridging the gap between the discipline's emergent understanding of the complexity of the concept and the methodological approach by which it is measured. It also seeks to bring the output of disaster resilience research in geography (which has historically commanded strong influence on policy) in line with the ongoing shift of focus in emergency management/ disaster preparedness from top-down, government-centered intervention policy which focuses on fixing deficiencies to bottom-up, place-specific policy which focuses on 
building on existing capacities and unique strengths. Ultimately this research is geared to benefit local emergency management personnel, civic leaders and citizens who take on the responsibility of fostering resilient characteristics within their communities. 


\section{CHAPTER 2. LITERATURE REVIEW}

\subsection{Hazards, Disasters and Vulnerability in the Social Sciences}

Attempts to construct a concise history of hazards research which is both comprehensive in scope and charitable to a wide-range of theories seems bound to fail. In terms of scope, it has been noted that even the longest of scholarly reviews fails to include all branches of thought where the topic receives treatment (Downes et al. 2013). In terms of theory, the number of active paradigms within hazards research is probably at least as large as the number of importantly related yet less explored theoretical linkages with other schools of thought. Even when limited to the urban context and the scale of communities, a surprisingly broad body of literature can be found. Yet, the story of the rise, maturation and widespread influence of hazards research is also, in many ways, the story of the rise, maturation and widespread influence of geographic theory in academia as well as in government decision-making.

For well over 60 years in America, scholarship across the social sciences has been directed toward assessment of urban populations' natural disaster vulnerability and in answering, for the benefit of academic as well as government audiences, the fundamental questions of 'Who is vulnerable to extreme natural events?' and 'What factors influence disaster vulnerability?' While conceptual frameworks in the study of vulnerability have evolved considerably throughout the post-war period, a sense of urgency for actionable research in this area has increased greatly in recent years. An illustration of the current relevance and complexity of the topic can be seen in the 
National Research Council's (2012) report, commissioned by the U.S. intelligence community, on the national security implications of increasingly frequent/intense extreme weather events due to global warming. In this report, the NRC recognizes that social, economic and political drivers of disaster vulnerability are not well understood, calling for intensive place-based research into the interplay of these factors that incorporates predictive models of all possible environmental hazards.

Disaster research in general in the social sciences prior to World War II has been regarded as sporadic, empirical and focused on singular events, garnering little attention within the researchers' respective disciplines and containing few theoretical contributions or methodological prescriptions that would suggest further study was promising. $^{2}$ Still, it is worth noting that these early contributions originated from across the social sciences - a harbinger of the developments of the post-war period and in today's multidisciplinary disaster research climate. One of the first attempts to apply accepted social science concepts to the study of a disaster was a dissertation in political science by Samuel H. Prince (1925), which examined demographic changes in Halifax, Nova Scotia following a devastating 1917 munitions depot explosion that leveled much of the city. In the early 1930's, W.M. Davis (1934) examined infrastructure damage in the Los Angeles region caused by the 1933 Long Beach Earthquake and evaluated the reconstruction recommendations made by a panel of California Institute of Technology

\footnotetext{
${ }^{2}$ An important exception is the human ecology paradigm presented by Barrows (1923), which provided theoretic context for several schools of disaster research in the geographic tradition
} 
scientists. $^{3}$ In the field of psychology, much attention was paid to the trauma incurred by U.S. military in the European trenches of WW I (Ferenczi 1921). Kutak (1938) reviewed the psychological impacts of destruction in Louisville, Kentucky following major flooding in 1937. Whatever the effect of these infrequent contributions on scholarly interest in the study of disaster, sustained treatment on the topic was not realized until after WW II, when Gilbert White and other geographers at the University of Chicago, through an examination of flooding disasters, established a paradigm of vulnerability which remained dominant until the 1980s (White and Kates 1978).

Influenced by the human-ecology paradigm in geography established by Barrows $(1923)^{4}$, White's 1942 dissertation Human Adjustment to Floods ${ }^{5}$ represented not only the first theoretical examination of factors of disaster vulnerability but also heralded a broad disciplinary shift toward behavioral science within geography. Influenced by the 'interventionist' environmental engineering policies enacted under The New Deal, as well as the American pragmatic philosophy of John Dewey $(1925),{ }^{6}$ White defined a range of 'adjustments' humans can employ to mitigate effects of flooding, in order to continue settling in flood-prone areas. These adjustments, he argued, took the form of technological (e.g. hydraulic engineering, structure elevation) or policy (e.g. land use code, flood insurance) fixes that guarded human lives and property from environmental

\footnotetext{
${ }^{3}$ Davis ended his article with the declaration: "Life is full of hazards, and we must take our chances among them."

${ }^{4}$ Barrows' focus was explaining human adjustment, in the broadest sense, to the physical environment, and advocated for a nomothetic approach within geography to establish universal laws of human-ecology interaction

${ }^{5}$ Republished post-war in an expanded 1945 monograph

${ }^{6}$ Characterized by, above all, the epistemological idea that usefulness determines what counts as knowledge (James 1977)
} 
hazards. Communities lacking the correct mix of adjustments against these hazards were to be designated vulnerable to the extent that they are at spatial risk to flooding. Also, in an implicit rejection of the existing 'rational choice' model of individual decisionmaking behavior, White pointed out the apparently counterintuitive trend of residential development in floodplains with few adjustments in place. White concluded his dissertation by calling for new explanatory research into people's settlement decisionmaking $^{7}$ and for a comprehensive federal policy of flood abatement taking into account all possible adjustments.

To understand the immediate interest and long-term influence across academia and in government of Human Adjustment to Floods, the broader social context of its release deserves mention. The monograph was published during the post-war period of high demand for the practical application of science and technology in urban and regional planning. Also concurrently, as the U.S. military analyzed the human occupancy of cities potentially targeted by Soviet nuclear missiles, there existed a high demand for research that could inform federal policy for reducing the impact on infrastructure and society of nuclear strikes. It has been argued that both the technophile social climate and civil defense orientation of government helped jettison White's research to the forefront of the popular new area of disaster mitigation research (Quarentelli and Dynes 1977; Smith 2001).

Known variously as the behavioral or risks/hazard paradigm, the theory of hazard adjustment was subsequently developed by White and his students Burton and

\footnotetext{
${ }^{7}$ A direct influence to Simon's (1956) 'bounded rationality' theory in psychology
} 
Kates, who formed the core of a 'Chicago School' of disaster research in the geographic tradition. Studies under this approach were broadly concerned with identifying the distribution of environmental hazards, the range of adjustments available to individuals, and how people perceive and make choices regarding hazards at the individual level (Cutter et al. 2000). As such, adherents to the behavioral approach developed several methodological foci. First, high importance was placed in gathering data on the spatiotemporal characteristics of environmental hazards, in order to both model their interaction with population and to quantify their effects (White and Haus 1975;

Friedman 1975). Second, a strong commitment to physical and managerial control, aimed at containing hazard impacts through environmental engineering and land use policy, was maintained (White et al. 1978). Third, government-led emergency response planning was urged as a mitigation tactic (Quarantelli 1979). Finally, analysis of settlement decision by the individual and policy decision by government officials was recommended under the lens of the bounded rationality theory of behavioral science, in order to understand how information about hazards and risk are processed and acted upon (Dynes 1970; Mileti et al. 1977). Throughout the development of the behavioral approach, the terms risk and hazard were synonymous with the spatiotemporal distribution of extreme environmental events. Although vulnerability assessment under the behavioral paradigm was seen primarily as a qualitative/inductive process based on measuring human occupancy of hazard zones and measuring the extent of adjustments in place, White maintained that the basic theoretical rationale was that hazards are derived from interaction between nature and society, strongly affected by feedback in 
the form of adjustments (White et al. 1978). Methodologically, practitioners of the behavioral paradigm were highly concerned with mapping locations of hazard zones to delineate risk, and cataloguing adjustments made at particular locales through detailed case studies (Friedman 1975). Meta-analyses of these studies were in turn used to determine patterns of adjustments and provide indicators of their effectiveness in dealing with disaster events. The dominant method for assessing individual and official decision-making behavior took the form of surveys, aimed at correlating demographic data with settlement decisions/policy (Dynes 1970).

Beginning in the 1970's, a series of radical critiques to the behavioral approach arose from across the social sciences, challenging White's techno-centric approach and the validity of a theory of vulnerability based solely on exposure to extreme natural events without consideration for social, economic or political factors. In his seminal paper "Taking the Naturalness Out of Natural Disasters", O'Keefe (1976) headed the first strong attempt to refocus the field on the human drivers of vulnerability. He argues that political and economic struggles in any form are 'force multipliers' which increase vulnerability of certain populations and amplify the effects of disasters. Explaining that these struggles tend to limit options for adjustment (thus limiting certain populations' ability to cope with disaster), he called for future research to account for barriers to human agency in vulnerability assessments. Critiques by Waddell (1977) and Torrey (1979) followed, each questioning White's causal mechanism (environmental processes) of hazard - they sought to replace geophysical characteristics with a 'geography of 
social relations' as the governing factor of spatial extent and occurrence interval of disasters.

These initial critical works established what was called the political-economic or political-ecology approach to vulnerability, which was subsequently developed primarily by social science researchers with experience working in least-developed/ Third World countries or economically/politically disadvantaged communities in the U.S. Themes of critique of the adjustments paradigm can be easily discerned. First, political ecologists point to the over-exaggeration of the role of the individual in adjustments writings, which artificially forces studies to ignore larger political, economic or cultural factors. Second, the adjustments paradigm was seen as lacking appreciation for historical or structural features of society which may limit individual adjustment choice. Politicalecology is seen as well positioned to offer insights into these limiting structural features because of the presence of Marxist geographic theory (Paul 2011), which offered explanatory linkages between disaster vulnerability and the spread of global capitalism. ${ }^{8}$ Waddell (1983), for example, argued that disasters function to reinforce the gap between rich and poor by accelerating economic marginalization and forcing inadequate adjustment choices. Susman et al. (1983) attempted to prove that governmental adjustments tend to be funneled to upper-class communities using a case study of differential damage to Guatemala City after a 1977 earthquake, which the authors dubbed a 'classquake'.

\footnotetext{
${ }^{8}$ Structural Marxists, especially in the geographic tradition of Louis Althusser, sought to evaluate how different levels of social formation manifested themselves in different levels of social inequality - uneven effects of disasters provided evidence for this position and resulted in interest by Marxist thinkers (Benton 1984).
} 
As a fundamentally critical endeavor, the political-ecology approach did not seek to offer significant methodological alternatives or policy prescriptions. ${ }^{9}$ However, as Marston (1983) notes, political-ecology's important contribution to disaster research lay in its success in broadly validating the social, economic and political as factors to vulnerability, and in its alternative explanation of human behavior in relation to societal structures. As will be seen, all subsequent theoretical contributions to disaster research put one or all of these factors in context. However, this is not to suggest that social considerations were completely missing from disaster research prior to the politicecology paradigm. In fact, many researchers in the field of sociology have been engaged in disaster research since the late 1960s, most notably in the disaster behavior tradition of Dynes (1970), Quanterelli (1978) and Drabek (1986). According to Dynes (1970), disasters "represent types of uncertainties in which elemental forms of social processes are revealed...They provide the opportunity to observe the emergence of social structure under stressful conditions." Their approach was focused on behavioral aspects, in the vein of the molar/perception school of social psychology, in order to model individual behavior based on hazard perception. It may seem that a high potential for collaboration existed between researchers in both the sociological and political-ecology traditions, but as Alexander (1997) has shown, sociological developments in disaster behavior occurred in relative isolation. Indeed, a theme of compartmentalization in disaster studies across several disciplines, which complicates

\footnotetext{
${ }^{9}$ A notable exception was Emel and Peet (1989), who advocated for more qualitative vulnerability assessments based on class/social issues, and recommended mitigation solutions based on redistribution of wealth and resources in society, rather than rely on science and technology to control nature.
} 
efforts of synthetic literature review, developed in the 1970s/1980s and continues to the present day. For example, the field of emergency medicine, stimulated by funding from the U.S. Centers for Disease Control in the early 1980s, began producing research on the characteristics of post-disaster medical emergencies in the built environment, with the aim of identifying trends of disaster-related medical vulnerabilities and recommending medical relief adjustments (Alexander 1997). However, as Taylor (1984) has shown, these efforts were isolated from a strong concurrent disaster research culture in the fields of architecture and civil engineering, which were producing very detailed knowledge of the performance of urban infrastructure during many types of disasters. While mutual incomprehension and the tendency to assert disciplinary relevance/identity have been blamed for a lack of interdisciplinary collaboration in vulnerability study (Carrerra and Guzzetti 1993), the problem of bridging these gaps remains to be solved.

Beginning in 1990, conceptual frameworks for studying vulnerability which included social, political and economic considerations developed rapidly in the social sciences. First, a 'hazards-in-context' approach, developed by Mitchel et al. (1989) and Palm (1990), was an attempt to expand White's behavioral and environmental approach to include social and political factors, by placing individual choice as the end product of a complex, multi-scale network of social/political power relations. Palm (1990) identified three scales - micro (individual/household), meso (emergency managers, insurance companies etc.), and macro (the State) - through which information about environmental hazards and adjustments are filtered. She demonstrates that 
political/social biases at the meso and macro level produce vulnerabilities at the micro scale by perpetuating misinformation ${ }^{10}$.

The next theoretical model of vulnerability in the social sciences, known as the pressure-release model, was devised by Blakie et al. (1994) and extended the causality critique of political ecology. The concept proposed was a progression of vulnerability from root social causes (poverty, lack of resources) to 'dynamic pressures' (population growth, inflation) to unsafe conditions (inadequate building codes, lack of emergency response plans) and finally to the disaster event itself. As a fundamentally humancentered approach, this model encouraged research to uncover all types of social, political and economic pressures that interact at the individual scale to create vulnerability.

A few human geographers in the place-based tradition, sensing a lack of placespecific study in either the pressure-release or hazards-in-context approaches, began seeking to bridge the behavioral and political-ecology paradigms in a conceptual framework known as hazards-of-place. Influenced by Hewitt and Burton's (1971) definition of vulnerability as the totality of hazards that could affect a particular ecological system, Cutter and Solecki (1989) proposed combining all-hazards research with studies of place-specific social and political structures to create 'hazardscapes' or 'riskscapes'. The goal was to synthesize environmental hazards with human systems at various geographic scales in a manner that allowed for the integration of data for quantified place-based vulnerability assessments (Cutter 2000). While hazardscapes

\footnotetext{
${ }^{10}$ For example, insurance companies can manipulate information about geophysical risk in order to artificially increase an individual's perceived risk
} 
were initially described in static language, subsequent development has provided a more fluid and dynamic view, in which both hazards and vulnerability undergo constant and complex spatiotemporal change (Khan and Crozier 2009). The idea of hazardscape in the geographic tradition is now concerned with identifying aspects of process, people and place (which geographers have always been attune) which shape hazards and human vulnerability. A salient characteristic of the hazards-of-place paradigm is its explicit goal of combining the strengths of earlier adjustments research (i.e., scientific data collection) and political-ecology (i.e., explanation of socioeconomic processes). Proponents of the hazards of place approach contend that it allows for empirical examination of patterns of environmental hazards while directly accounting for the interplay of quantified social, economic and political factors. Especially significant to the rise in popularity of this approach was Cutter's early recognition of geospatial technologies as key tools in evaluating hazards, analyzing population characteristics and communicating issues of risk and vulnerability to decision makers.

With rapid advances of the aforementioned geospatial technologies such as geographic information systems (GIS) and remote sensing in the last decade, social scientists have been presented with increasingly sophisticated tools to visualize environmental hazards, map risks/vulnerabilities, and model disaster impacts (Vogel et al. 2007). The hazards-of-place paradigm, with its emphasis on local empirical research, has provided disaster vulnerability experts the theoretical underpinnings for applied research in this area. Aiming to enable researchers to leverage geospatial technologies in their research while remaining amenable to accepted standards of scientific praxis, 
researchers have recently proposed and developed guidelines for an applied 'science of vulnerability' (Cutter 2003; Vogel et al. 2007). Adhering to and acknowledging the basic critiques of spatial science, ${ }^{11}$ a science of vulnerability would incorporate foci that may mitigate against some of the perceived counterproductive effects of scientific praxis.

These foci include (Cutter 2003):

- Multiperspective reflexivity - the ability to learn from a variety of knowledge sources, both expert and lay, in a place-based all-hazards approach

- Transdisciplinary linkage - the ability to actively seek connections across the social and natural sciences (including health and engineering disciplines)

- Methodological pluralism - the ability to integrate multiple research methods and technologies in recognition of place-specific variability of vulnerability

As an explicitly pragmatic applied approach, vulnerability science has developed to encourage the exploitation of geospatial technologies for analysis and visualization of hazards data, with the purpose of helping emergency management officials understand vulnerabilities of places and enact more effective mitigation policies (Tobin and Montz 2004; Rufat 2012).

\subsection{Evaluating Resilience}

While the academic debate described above dealt primarily with defining what exactly makes populations vulnerable to environmental hazards, the concept of resilience explores the characteristics of systems, both physical and organic, which seem to protect against the harmful effects of extreme environmental events. Possibly

\footnotetext{
${ }^{11}$ What Cutter calls the 'vulnerabilities of science' - the bias of the supposedly objective observer, the social construction of science and scientific practice, the tendency of the dominant modes of scientific discourse to exclude certain perspectives and knowledge sources, etc.
} 
due to the antonymic coupling assigned to the two terms in English semantics, 'vulnerability' and 'resilience' may seem simple opposites on a scale of disaster readiness. Though related, the two concepts are often understood to refer to different processes, with the study of resilience undergoing mostly separate development in several different disciplines.

A very general way to capture the tone of the development of resilience thinking across the social and natural sciences may be a shift from focusing mostly on deficits, weaknesses and vulnerabilities (whereby system agents are acted upon by extreme events), to an inclusion of focus on adaptation, anticipation of change and learning (whereby system agents are proactive and preemptive in dealing with environmental threats). Key traditions of inquiry into the resilience concept as it relates to human systems and communities are now reviewed.

\subsubsection{Psychological Sciences}

In the 1970's, case studies examining differences in individual's responses to environmental shocks, including disasters, prompted a longstanding theoretical tradition of resilience thinking in the fields of developmental and social psychology. This tradition had early on rejected notions of inherent or genetic 'invulnerability' to environmental shocks, as well as focus on deficits of the individual (the so-called risk paradigm of developmental psychology of Boyden and Cooper (2007) and Johnson and Wielchelt (2004)). Instead, this branch has focused on identifying strengths and enabling the development of social competencies and internal coping mechanisms 
identified as 'functional resilience' by Howard et al. (1999) and Luthar and Zelazo (2003).

A lasting hallmark of the psychological approach, contributed by eminent psychopathologist Norman Garmezy, is that an individual's level of functional resilience does not have to be exceptional or optimal in order to successfully navigate a crisis; it just has to be relatively better than others experiencing the same level of environmental shock (Garmezy 1991; Rutter 2012). Social psychology, as a sub-discipline which seeks to bridge individual-level psychological processes with the development of social structures, has in the last three decades explored the components and processes of community resilience. This body of research often focuses on the family and the neighborhood (Downes et al. 2013), and broadly defines resilience as a dynamic process encompassing positive adaptation within the context of significant adversity (Luther et al. 2000). Dimensions of community resilience identified in social psychology include: strategic self-organization (Sherrieb et al. 2010), strong people-place connection (termed place identity by Putnam (2000)), mechanisms for information sharing, strategic social networking (Obrist et al. 2010), connection to government entities (Ungar 2011), economic diversification (Attaran 1986), and others. Though there may seem significant theoretic overlap between resilience thinking in ecology and social psychology, there was in fact little cross-reference between these disciplines in last decades of the $20^{\text {th }}$ century, and each remained somewhat compartmentalized in their research (Berkes and Ross 2013). By the mid-1990s, a natural disaster-specific strand of resilience thinking had emerged within social psychology which was primarily focused on examining how urban communities, especially at the neighborhood level, engaged in resilience-building 
activities (Paton and Johnston 2001). For the resilience-building process to be successful, this research identifies three key qualities. First, the process should be intentional, strategic and collective (Norris et al. 2008). Second, it should be emergent from within the community, not primarily motivated or driven by outside actors (Clark 2006). Third, it should assume and be able to cope with continual environmental change, as well as with uncertainty, unpredictability and surprise (Goldstein 2009).

\subsubsection{Physics and the Physical Sciences}

Often overlooked in the social sciences, there exists in physics a strong tradition of inquiry into the nature of extreme natural events and the quantitative components of resilience. Breakthrough discoveries of the statistical properties of simulated nonequilibrium systems in the 1980s, which described the apparent self-organizing behavior and certain universal statistical similarities of such systems (including critical points, fractal geometries and scale-invariant functions), enabled physical scientists in many disciplines to develop theoretic models of complex/nonlinear systems. Crucially, these new discoveries provided the mathematical basis for viewing extreme events and phase shifts as natural and ubiquitous in 'the real world'. Findings of lab experiments in socalled self-organizing criticality (SOC), which actually involved statistical modeling of 'avalanches' occurring in piles of sand, soon found wide application in describing the characteristics of extreme events in many identified complex physical and socioeconomic systems, including: distribution of earthquakes (Schotz 2002), sudden fluctuations in financial markets (Cont and Bouchaud 2000), traffic jams (Helbing 2001), 
the spread of epidemics (Stollenwerk 2005), behavior of forest fires (Song et al. 2001) and many others. While some disciplines such as seismology have tended to apply SOCinspired theory to explaining the extreme event hazards in systems, others such as computer science and network science also study what makes systems and system components resilient to the effects of extreme events, phase shifts or system failures. In computer science, for example, the assumption or expectation of extreme disruptive events inherent in SOC-related theory has given rise to research in fault-tolerant systems (also called graceful degradation), which has resulted in robust computer system configurations that can cope with various types of failure or error (Carlson and Doyle 2002). In the network sciences, some researchers have studied how the outcome of a disruptive event (i.e. a targeted or untargeted attack) on a system relates to the structure of connections among system components (Matisziw et al. 2012).

\subsubsection{Ecology and Social-Ecological Systems Theory}

Though the word resilience appeared earliest in academic discourse from within psychology (in reference to now-defunct theories for preventing psychological trauma in people believed to possess a genetic risk to psychopathologic mal-adaption), most scholars in both the social and natural sciences now agree that the current understandings of resilience are derivative from ecology (Waller 2001; Moser 2008; Boon et al. 2012), especially the work of C.S. Holling beginning in the 1970s. By Holling's time, traditions of linear thinking about ecological systems, the hallmark of which involved organisms bound to a single stable state, had begun to give way to influential 
new complexity theory, which asserted nonlinear relationship between system variables, multiple possible/alternative system states (which constituted attractors to system variables), unpredictable system perturbations, and emergence, or the selforganizations of system components (Zadeh 1976).

Holling et al. (2002) asserts that both current and alternative system states were never static and underwent constant change, based upon their research on how ecosystems with a certain biologically-favored function, structure, identity and/or feedbacks were able to retain those elements subject to various perturbations. He used the term resilience to describe the capacity for biological systems to remain within the thresholds necessary to retain such favored elements, while at the same time adapting to ever-changing environmental conditions (Pendall et al. 2010). Though Holling's initial inspiration for theorizing about resilience were the complex ecosystems of boreal forests, with their disturbance mechanisms, species regime shifts, and cycles of renewal, his influential work quickly prompted interest in conferring his theorized principles of ecological resilience to human systems (Gunderson 2000; Bhamra et al. 2013;). The resulting multi-disciplinary study of social-ecological systems (SES) examines the feedback loops and interdependencies of linked human and natural systems, particularly in order to identify and explain the various behaviors, structures, relationships and processes that humans employ to build resilience to socio-ecological hazards.

A common thread which runs through the elements of resilience identified under the SES paradigm is the importance of adaptation to change, a quality seen by ecologists 
as crucial to the navigating continuous cycles of renewal and recurrent disturbance mechanisms. While some adaptive capacities of human systems are identified as physical qualities or structural characteristics (which may be seen as resistant to the effects of disturbance mechanisms and environmental hazards), the SES perspective highlights capacities in terms of the strategic behaviors, choices and relationships established by humans which enable direct interaction with hazards while lessening the risk of those hazards pushing human systems into an unfavorable state. This is in line with ecology's assumption of inevitable interaction between organic systems and environmental hazards. Further, this view provides SES scholars theoretical space for explaining certain human behaviors which had heretofore seemed enigmatic (especially in academic psychology), such as intentional settlement in flood-prone areas (James and Hall 1986), willingness to allow infrastructure to fail during an extreme event (Brinn and Greaves 2003), and 'giving up density' in certain resources as a tradeoff to exploit new resources (Morgan and Brown 1995).

The SES paradigm devotes considerable attention to the adaptive renewal cycle, a heuristic model meant to depict the cyclic patterns of biological potential, growth, disturbance and reorganization which defines the SES conceptual model of ecological systems. Figure 1 depicts the adaptive renewal cycle, showing how biological agents adjust their strategies during four conceptual stages, and how the level of agent connectedness and potential relate to these four stages. Recognizing that multiple 


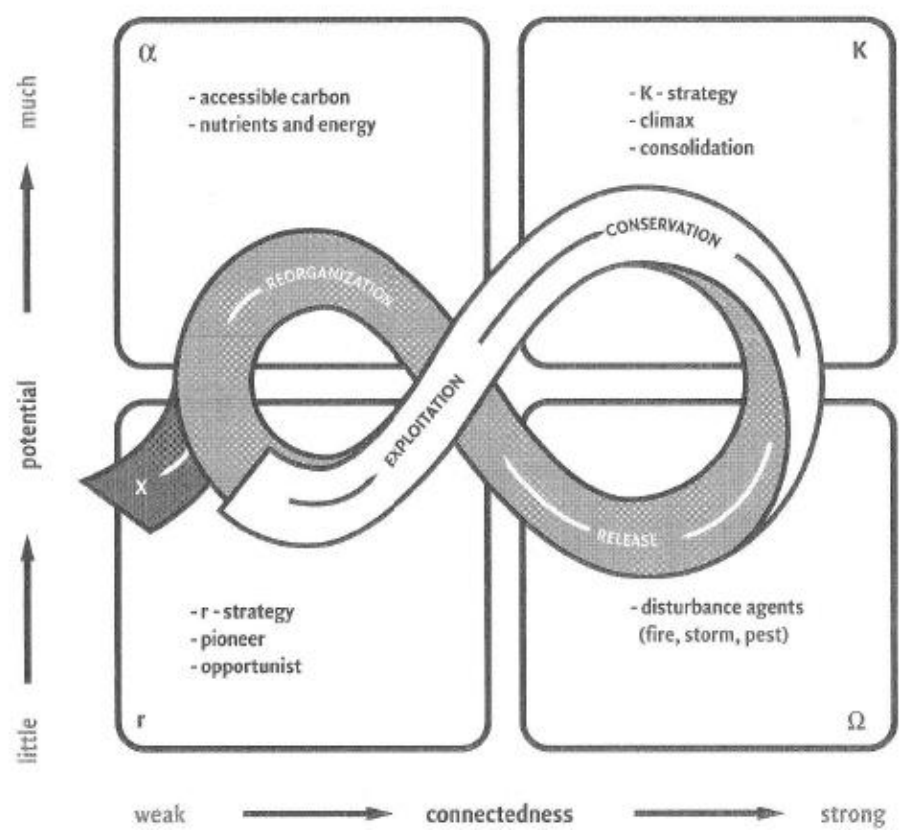

Figure 1. The adaptive renewal cycle within the Socio-Ecologic Systems (SES) paradigm depicts 4 thematic Quadrants (and related biological strategies) and shows the relationship with 'connectedness' ( $x$ axis) and 'potential' (y axis) (Berkes et al. 2003)

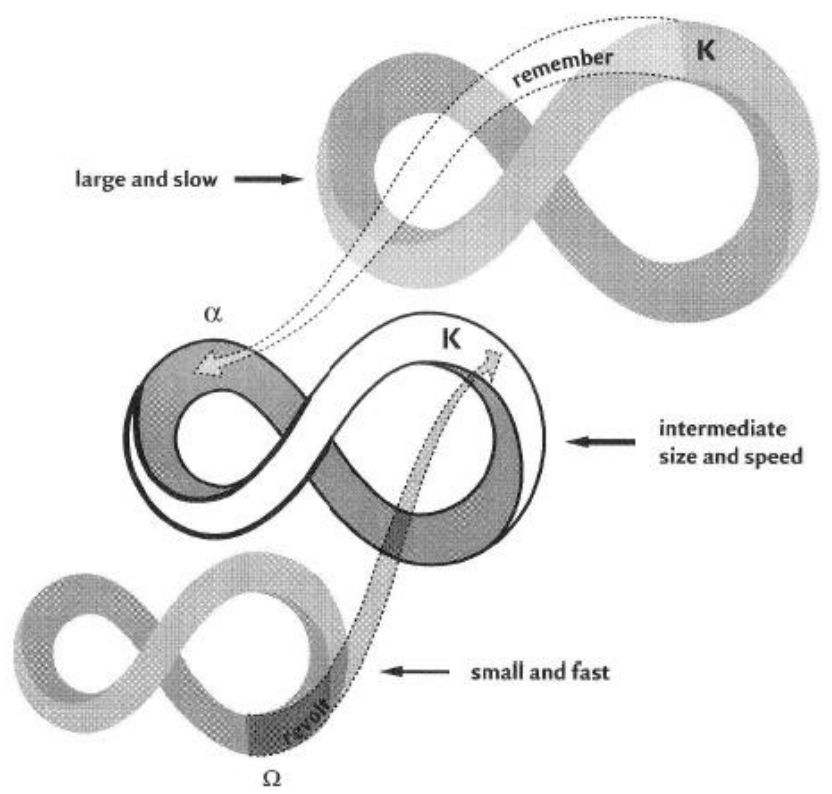

Figure 2. The structure of feed-back and feed-forward influences among multi-level renewal cycles constitutes one of the mechanisms by which complexity is introduced into such systems. Nested cycles at the micro, meso and macro level may be interpreted as representing individual-, community- and government-level processes, respectively (Berkes et al. 2003) 
cycles of growth, disturbance and reorganization may be acting on an ecological simultaneously (but at different spatiotemporal scales), SES theory uses another heuristic depicting multi-scalar structures of adaptive renewal cycles. Operating simultaneously, the processes at each scale are assumed to influence each other through feedback loops. Figure 2 depicts these nested structures and feedback loops, at a micro, meso and macro scale (interpreted here as perhaps representing the individual, community and government level within a human socio-ecological system) which can be associated with fast, medium and slow change, respectively. SES identifies a crucial consequence of this nested feedback structure as the idea of panarchy, in which system agents/processes at one scale may influence (or be influenced by) agents/processes at other scales; this being one of the mechanisms by which non-linearity, uncertainty and surprise are introduced into complex systems.

Scholars across several disciplines have attempted to formalize the properties which distinguish complex systems from other types of conceptual models within broader systems science, often operationalizing the properties to suit the researchers' discipline (Zadeh 1976; Sterman 2000; Larsen-Freeman and Cameron 2008;). Berkes et al. (2003) identify five attributes of complex systems in the context of SES theory:

1) Non-linearity: The various (and often unpredictable, counter-intuitive or surprising) co-influence among system variables at different levels (nested feedback and feed-forward relationships) necessitate descriptive techniques that combine qualitative, possibilistic statements with quantitative, probabilistic ones. As such, mathematical equations which describe complex systems rely on differential calculus to enumerate 
many possible stability domains or attractor states which could manifest given current known system conditions. ${ }^{12}$

2) Uncertainty: As a consequence of the non-linear mathematics of complex systems, information regarding the changes in system conditions cannot produce quantitative/probabilistic measures of 1) which attractor state/stability domain the system is heading towards, 2) the rapidity which the system is moving towards such state, or 3) the proximity in space or time to that state. This leads to inherent uncertainty and surprise concerning the magnitude of condition parameter changes and/or timeframe which could initiate a shift in system states.

3) Emergence: Within complexity science, system agents are not theorized to passively experience changes to system conditions. Further, the (seemingly) random physical forces which elicit changes in systems (either reinforcing the current stability domain or pushing the system toward another attractor state) are not understood to lead to random or unstructured relationships among system agents. Instead, changes in system conditions are theorized to provide a platform for, or to enable, structured behaviors and connections among agents manifesting in patterns of accumulating change. Further, it appears to complexity scientists that this structured, accumulating change (which is taken to be synonymous with growth) is directed or oriented toward a certain stability domain - most often the system's current domain. Resilience can be understood to be an emergent property within a system, or an aggregate of emergent

\footnotetext{
${ }^{12}$ Differential equations, in this sense, start from current conditions and work 'forward' (on the left side of the equal sign), generating possible 'stability domains' which the system could move toward. A related tool within complexity science is the Fourier Transformation, which starts from current conditions and works 'backward' (on the right side of the equal sign'), enumerating all possible (theoretically infinite) equations which could make up and therefore lead to the current conditions.
} 
properties, directly referring to behaviors and connections among system agents that serve to sustain/exploit the current stability domain during 'normal' conditions, or seek to preserve as many structures of accumulating change (growth) as possible during periods of (sometimes rapid, unforeseen or disruptive) shifts to other stability domains. In this way, SES theory uses emergent resilience as a scaling concept to make the seemingly infinite complexity of complex systems more manageable: it is simply an overall characterization of 1 ) the extent to which agent behaviors and agent interconnections are oriented to the current stability domain or another attractor state (answering the question 'Resilience for what? ${ }^{13}$ ), and 2) to what extent such behaviors and connections are configured to mitigate certain system forces (e.g. disturbance mechanisms, environmental hazards) which threaten built-up growth structures or push the system toward an unfavored state (answering the question, 'Resilience to what? ${ }^{14}$ ). Crucially, Berkes et al. (2003) asserts that as a consequence of the nonlinearity and uncertainty properties of complex systems, characterizations of resilience must necessarily be qualitative. Resilience is seen to emerge at different levels of systems as a tentative and constantly evolving defense against an uncertain future.

4) Self-Organization: SES theorists operationalize an interesting finding from statistical physics on the overall behavior of highly dynamic, nonlinear (complex) systems - that such systems seem to organize spontaneously and automatically around so-called critical point attractors (Bak et al. 1987) - by asserting that all emergent processes are also self-organized. Critical point attractors are interpreted as critical

\footnotetext{
${ }^{13}$ Carpenter et al. 2001

14 Ibid.
} 
points of instability (or opportunity) which system agents (through emergent processes) will automatically organize around within the aforementioned adaptive renewal cycle (Figure 1). Moreover, SES theorists extend finding from physics and control theory that self-organizing behavior in simulated dynamic systems occurs regardless of the parameterization of system variables - such behavior seems to emerge whether variables are finely tuned throughout a simulation or even reset to random values. SES scholars take this to mean that self-organizing behavior is inherently natural behavior which occurs outside the confines of rigidly controlled laboratory experiments.

5) Scale: An important consequence of the concept of nested, multileveled systems, each with their own emergent properties and each influencing processes at other levels, is that analysis of such systems should be conducted simultaneously at different scales, including the micro, meso and macro levels. In discussing scale in complex systems, SES theory returns to the core focus of the paradigm: how human social systems are affected by and influence natural systems (and vice versa), and how humans can achieve environmental management outcomes and build resilience to natural hazards. When problems of biodiversity conservation, for example, are considered, it becomes apparent that solutions at the genetic level can be quite different than those at the species or ecosystem level, and that actions taken at each level influence all others through feedback loops (Gunderson and Holling 2002). This finding highlights the importance of multi-scale analysis for effective policy and management decisions. 
2.2.4 The Shift of Focus From Community Vulnerability to Community Resilience As previously reviewed, inquiry into human disaster resilience began within ecology and resource management in the 1970s, drawing inspiration from observations on how systems of organisms cope with and respond to environmental disturbance mechanisms (i.e. hazards) and/or overwhelming extreme events (disasters) (Paul 2011). Spurred by early concern for the potentially negative biodiversity effects of climate change, this research, proceeding under a systems theory praxis, attempted to describe and model the various preventative and alleviative structures deployed by an ecologic system to prevent failure as a result of interaction with an extreme event (Holling 1973). Led by Holling (who argued that resilience, coming from the Latin resilio meaning 'to bounce back', was the appropriate overarching term), ecological resilience enjoyed continued theoretical development through the 1990s, when its focus on biophysical attributes such as functional diversity, component redundancy and damage resistance mechanisms began garnering attention of the larger hazards research community.

The policy recommendations of this research community had, until the late $20^{\text {th }}$ century, followed the general top-down theme of encouraging government-led interventions into the drivers of vulnerability (Paul 2011). This focus on vertical hazard adjustments, originally championed by Gilbert White (1945) and embraced in government during the 'Civil Defense' era of the 1950s and 1960's, was further stimulated in the 1990 s by several major national and international disaster mitigation initiatives, which challenged governments at all levels to reduce populations' risk to death, injury and economic loss from natural disasters. Most notably, the UN General 
Assembly's declaration of the 1990s as the International Decade for Natural Disaster Reduction (IDNDR) prompted a broad refocusing of research agendas toward supporting official organization and administration of economic development, hazard preparedness, and response/recovery programs (Montz et al. 2003). In this atmosphere, US government interest and funding of hazards/vulnerability research (with its strong tradition of identifying opportunities for government-led adjustments) outpaced related work in the ecologic resilience tradition (which focused on what makes organisms resilient to extreme events) (Paul 2011).

However, in the wake of Hurricane Katrina in 2005, the emergency management policy community has recently shifted its focus to identifying and analyzing factors which generally make communities more resilient to any environmental hazard. As evidenced in the National Science and Technology Council's 2005 reports on the 'Grand Challenges of Disaster Reduction', this policy realm is focused on characterizing and fostering community resilience, under the assumption that if resilience can be increased, communities will be more likely to withstand or recover from an extreme event. At the federal level in the United States, this shift has found institutional acceptance in the creation of the Office of Resilience within the National Security Council. Here can be discerned an 'official' opinion, at least in federal emergency management policy circles, of the direction hazards research should take - instead of research which is oriented to fixing vulnerabilities, federal policymakers are currently preferential to research which can help build resilience (Cutter et al. 2010). Many academics whose publications are directed at policy audiences have duly noted this 
emerging trend and have refocused their research agendas accordingly, to the point that resilience research is currently viewed as particularly trendy (Manyena 2006; Pendall et al. 2009; Reghezza-Zitt et al. 2012). This has in turn brought previous paradigms of community resilience, such as the socio-ecological resilience perspective of Holling, back onto the radar of geography, with new conceptual frameworks of community disaster resilience commonly integrating concepts such as redundancy, coping capacity and adaptive learning (Tierney and Bruneau 2007; Gunderson 2010).

It is worth noting that the concept of community disaster resilience is approached differently between the quantitatively-oriented researchers and human geographers (influenced by political ecology, sociology, etc.). In what may be termed a structural paradigm of resilience within the quantitative camp, community resilience is often defined as an outcome of aggregated baseline system states which, if present in a community, tend to create resistance to system disruption and/or allow efficient return to normalcy. This research is often concerned with benchmarking, for example, a community's road infrastructure redundancy to characterize evacuation potential, or analyzing a local EMT district's medical supply contingency plans. This structural approach often heavily utilizes spatial data in a GIS environment, sometimes including data from remote sensing systems to infer system states. On the other hand, the human geography camp may be seen to take on a more functional view, often framing community disaster resilience as a multi-scale process (systems within systems) which continually effect resilience. As a paradigm which focuses on process rather than outcome, this functional approach often analyzes the spatiotemporal change of 
demographic variables which are known to influence resilience, such as community diversity and employment sector dependence.

Table 1. Differences between two broad paradigms of disaster resilience

\begin{tabular}{|c|c|}
\hline Structural & Functional \\
\hline Resilience is framed as an outcome & Resilience is framed as a process \\
\hline $\begin{array}{c}\text { Focused on benchmarking system } \\
\text { states (redundancy, contingency, etc) } \\
\text { that affect community resilience }\end{array}$ & $\begin{array}{c}\text { Focused on analyzes multi-scale } \\
\text { processes (systems within systems) } \\
\text { that affect community resilience }\end{array}$ \\
\hline $\begin{array}{c}\text { Often utilizes remotely-sensed } \\
\text { environmental data and spatial } \\
\text { statistics in a GIS environment }\end{array}$ & $\begin{array}{c}\text { Often utilizes demographic variables } \\
\text { as indicators of resilience }\end{array}$ \\
\hline $\begin{array}{c}\text { Common in urban planning, } \\
\text { architecture \& civil engineering }\end{array}$ & $\begin{array}{c}\text { Common in geography, social } \\
\text { psychology \& ecology }\end{array}$ \\
\hline
\end{tabular}

A new integrative conceptual framework of community resilience, pioneered by researchers at the Hazards and Vulnerability Research Institute at the University of South Carolina, has been proposed to bridge the theoretical gap between the structural and functional viewpoints (Cutter et al. 2010). Termed the Disaster Resilience of Place (DROP) model, it places emphasis on the antecedent conditions of a community's social, natural and built environments, which can in turn be analyzed as 'systems within systems' that are continually evolving. When these baseline antecedent conditions interact with a hazard event, the characteristics of a community's short-term coping mechanisms and long-term absorptive capacity, which themselves can be analyzed as system states, determine whether and to what extent the hazard event becomes a 
disaster. The DROP model places importance, regardless of the actual outcome of the hazard event, on whether or not a community demonstrates learning behavior from the event in the form of mitigation and preparedness measures. This

Figure 3. Heuristic Representation of Disaster Resilience of Place (DROP) Model

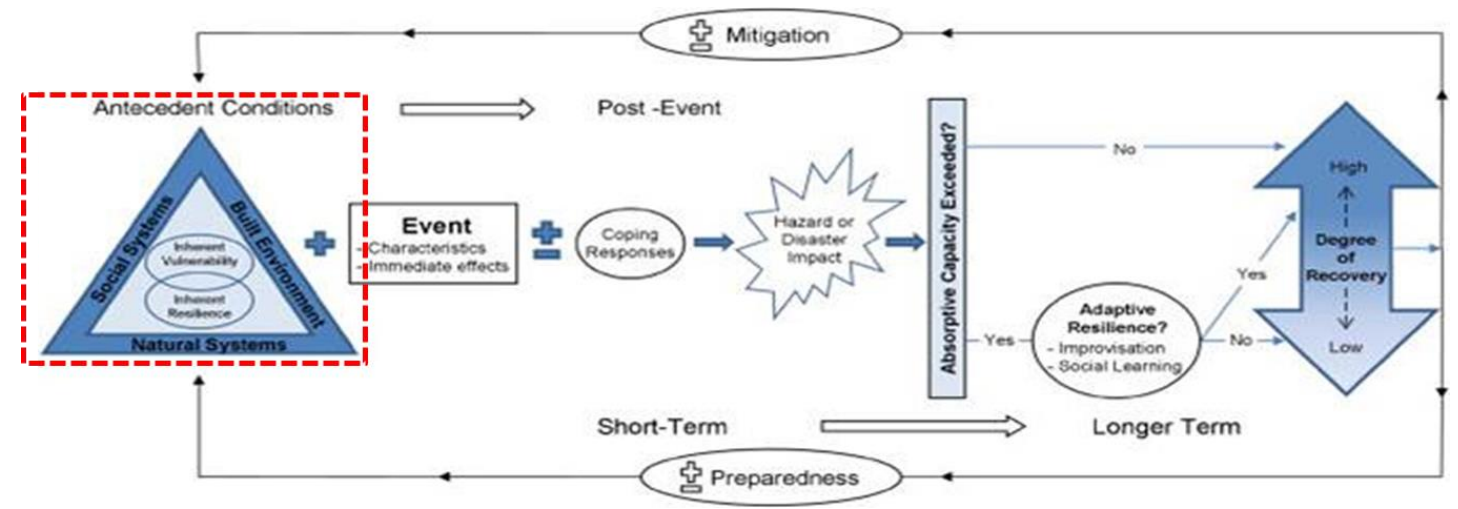

Source: Hazards \& Vulnerability Research Institute, Cutter et al. (2005)

creates either a positive or negative feedback loop in which the strength or weakness of mitigation and preparedness becomes part of the antecedent conditions to be tested by the next extreme event.

The DROP model has provided an exciting chance to integrate methods and perspectives from across the social and natural sciences to characterize community disaster resilience, as it recognizes the existence and importance of complex feedback mechanisms among the various components of resilience. However, recent applied research which identifies the DROP model as its conceptual framework often selects measurement methods (such as simple additive/aggregate indices) which fail to account for mutual influence and cause-effect relationships among these components. Such 
studies are also prone to relying disproportionately on individual-level variables, often derived from Census data in the United States, to represent community-level processes, qualities or dynamics. Further, there exists in current geographic community resilience research a tendency to select study units which aggregate variables to large, heterogeneous spatial units, a practice which may hide or mischaracterize the variability of resilience within specific places. Each of these identified methodological shortcomings will be examined individually.

\subsection{Geographic Indices of Resilience}

Conceptual models such as indices of socioeconomic and environmental variables serve an important role in helping many disciplines to understand natural hazards, disasters and resilience (Tate 2012). In cases where many variables can be identified to make up the process to be modeled, aggregated or additive indices have a long tradition of use in the social and natural sciences (Parris and Kates 2003). Figure 4 depicts an example of a composite index with two sub-indices (Social and Economic) and example component indicators/variables. However, the dominant methodological choices for constructing and analyzing such indices does not take into account the interrelationships and co-influence among the demographic and socioeconomic variables commonly utilized in resilience studies (Hiete et al. 2012; Rufat 2013). In fact, the most common forms of index construction (additive indices of the deductive, hierarchic and inductive form as shown in Figure 5) retain the mathematical assumption of independence among variables (Jones and Andrey 2007; 
Figure 4. Example of composite index construction

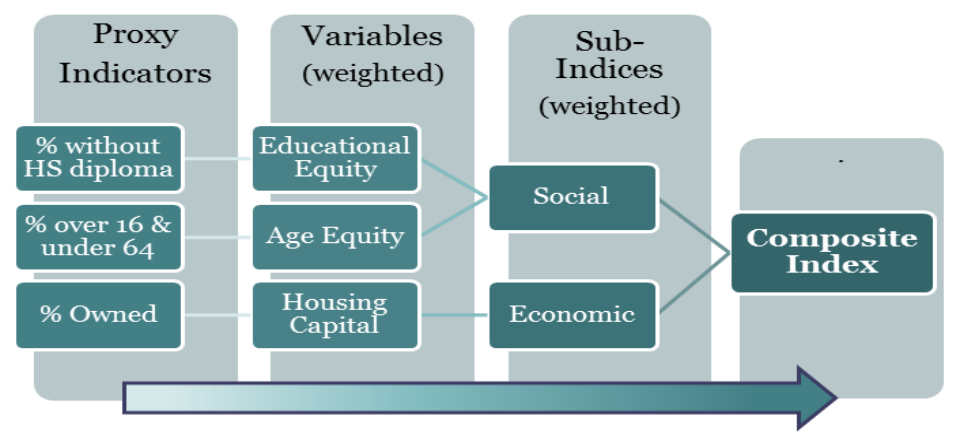

Figure 5. Structure of 3 Common Additive Indices (Tate 2012)

(a)

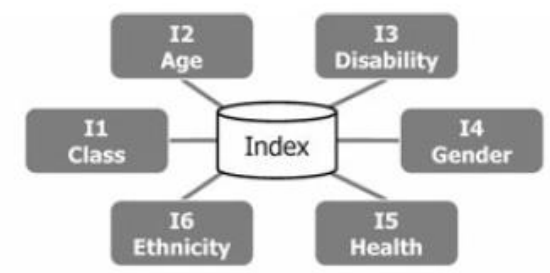

(b)

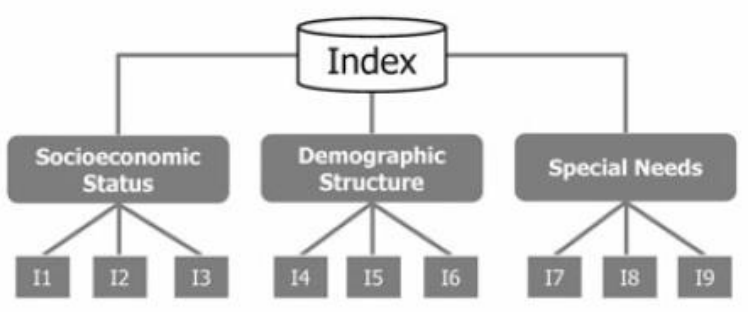

(c)

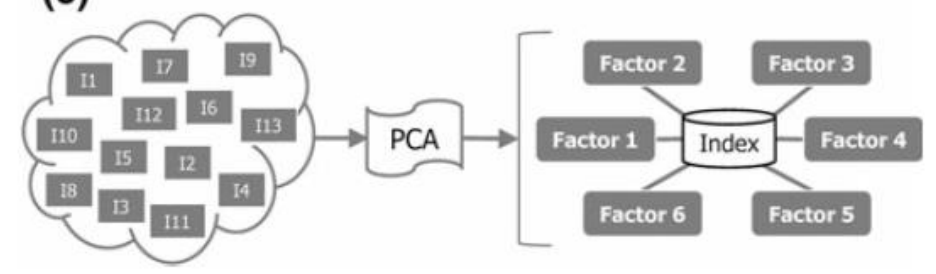

a) The deductive index assumes that component variables are ontologically discrete, independent of each other, and collectively representative of all system processes. Variables can be thought of as building blocks which, when assembled, comprise $100 \%$ of the system being analyzed

b) The hierarchical index assumes the same as the deductive, with the addition of categorizing sub-indicators into composite categories.

c) The inductive index assumes that some system variables may exhibit collinear statistical properties, and attempts to decorrelate an initial set of variables into a smaller set of factors which are as statistically distinct as possible. 
Hiete et al. 2012). As an analytic tool with a long history of use within systems science, the basic additive index attempts to model the totality of processes within a system by compiling all identifiable system variables (sometimes sorted into distinct categories) into an aggregate index (Jones and Andrey 2007). Table 2 depicts stages necessary to construct and analyze an additive index, along with the options geographers commonly select for such indices in current disaster resilience research.

Table 2. Stages of Additive Index Construction

\begin{tabular}{|c|c|c|}
\hline Stage & Description & Common Choice in Geography \\
\hline $\begin{array}{l}\text { Conceptual } \\
\text { Framework }\end{array}$ & $\begin{array}{c}\text { Vulnerability/Resilience dimensions to } \\
\text { include and their literature } \\
\text { justifications }\end{array}$ & $\begin{array}{l}\text { SoVi(Cutter et al. 2005), DROP } \\
\text { Model (Cutter et al. 2008) }\end{array}$ \\
\hline Structural Design & Organization of indicators within index & PCA-Corrected Hierarchical \\
\hline Analysis Scale & $\begin{array}{c}\text { Geographic aggregation level of } \\
\text { indicators }\end{array}$ & $\begin{array}{l}\text { US Counties or Census MSA } \\
\text { geographies }\end{array}$ \\
\hline $\begin{array}{l}\text { Variable/Indicator } \\
\text { Selection }\end{array}$ & $\begin{array}{c}\text { Variables and their proxy indicators } \\
\text { used to populate sub-indices }\end{array}$ & $\begin{array}{l}\text { Majority based on decennial } \\
\text { Census and ACS data }\end{array}$ \\
\hline Measurement Error & Discuss accuracy and precision of data & $\begin{array}{l}\text { Multivariate statistics; PCA } \\
\text { used to eliminate collinear } \\
\text { variables }\end{array}$ \\
\hline Transformation & $\begin{array}{l}\text { Proxy indicator representation } \\
\text { /manipulation into variables }\end{array}$ & Varies \\
\hline Normalization & $\begin{array}{l}\text { Standardization of variables to a } \\
\text { common measurement scale }\end{array}$ & Indicators normalized to [0-1] \\
\hline Weighting & $\begin{array}{l}\text { Definition of importance of each } \\
\text { variable and each sub-index }\end{array}$ & $\begin{array}{c}\text { Equal Indicator Weighting } \\
\text { Preferred }\end{array}$ \\
\hline Aggregation & $\begin{array}{l}\text { Combination of sub-indices into } \\
\text { composite index }\end{array}$ & $\begin{array}{c}\text { Equal Sub-Index Aggregation } \\
\text { Preferred }\end{array}$ \\
\hline Representation & Mapping analysis results & Regional-Level Maps \\
\hline
\end{tabular}

Note: Since variable independence is assumed in additive indices, a stage for identifying and accounting for variable inter-influence is rarely prescribed, and usually omitted in geography. 
When utilizing any form of additive index, researchers are free to decide to correct for, ignore, or otherwise account for the statistical similarity (co-linearity) inherent in component variables (although the dominant standards of rigorous scientific discourse dictate correcting co-linearity through internal multivariate statistical methods). In the case of the inductive additive index, statistical similarity among variables is addressed most often by eliminating variables not retained in the $1^{\text {st }}$ component of a principle component analysis (PCA). The eliminated variables are assumed to be redundant, and therefore less-informative and non-essential to the overall system model (Tate 2012). While such approaches have proven sufficient in modeling physical systems and mechanical processes, the extension of the same methodology to model human systems has been identified as highly problematic by researchers in the social sciences (Zadeh 1976, Barnett 2008), many of whom argue human systems are better described by the principles of complexity science (the study of highly dynamic, nonlinear, and complex systems). As mentioned, within complexity science, variables in dynamic systems are assumed to interact in non-linear (or even chaotic) ways, especially when examined at small (local) scales (Stern 2000, Berkes et al. 2003). Consequently, the interactions among many types of dynamic system variables resist quantitative description. Complexity scientists have explored ways to use qualitative or semantic descriptions in a knowledge-based approach to model these types of variable interactions (e.g. Zadeh 1976). Further, complexity science identifies an emergent (self-organizing) property of variables in such systems, one consequence of which is that variables cannot be assumed to be redundant, non-essential or non-informative based on solely their 
covariance (Berkes et al. 2003), which is the statistical property used in a PCA analysis (Abdi and Williams 2010). Also, complexity science observes that system variables may not be ontologically pure, unambiguously distinguishable from one another, or able to be parsed into discrete categories.

Lack of account for variable inter-influence when using additive indices to analyze disaster resilience in human systems demonstrates a lack of connection with multidisciplinary understandings of the complexity and non-linearity of the components of resilience, especially as illustrated within the SES paradigm of ecology. Luckily, methodological tools can be found within complexity-inspired disciplines which attempt to take into account the extent of variable interrelationships/co-influence, accommodate uncertain, vague or qualitative data, and integrate expert knowledge. One such tool is the Decision-Making Trial and Evaluation Laboratory (DEMATEL) method for uncovering both cause-effect relations and the most influential indicators among a large set, based on expert-system evaluation of indicators and applications of digraphs (Fontela and Gabus 1976). The core of the DEMATEL approach is to quantify complex indirect relations among variables based on perceived direct relations. Another is the analytical hierarchy process (AHP) (Saaty 1980, Zahedi 1986). Based on a digraph method complimentary to DEMATEL but geared toward capturing indicator preference in the expert-system phase (Hiete et al. 2012, Wu et al. 2012), AHP has proven useful for providing index weights based directly off expert opinion (Saaty 2001, Saaty 2008). Methods such as these not only account for feedback relations among variables, but they also provide decision makers with information about the causal 
nature of certain variables. This is a crucial aspect, because emergency management policy is more likely to have an impact if directed toward causal variables, or those which exert the most influence (Hiete et al. 2012). Further, these methods do not rely solely on the internal statistical nature of index variables to determine their information potential, and it eliminates the risk of rejection of important variables via a PCA analysis.

\subsection{Accounting For Uncertainty in Measures of Community Resilience} Complexity science and complexity-inspired academic paradigms have generally tended to resist absolute or rigid definitions of the phenomena they seek to describe. This is because the phenomena studied are ontologically vague and resist discrete classification. Consider four proverbial dimensions of human society-social, economic, political and cultural. Complexity scientists see these terms as fuzzy, in the sense that it is difficult to unambiguously assign the many identifiable components of human society into these four dimensions (to which dimension would one assign the component of religion, for example?). Add to this the complicating factor that complexity science sees components influencing each other through feedback mechanisms, and one begins to see the problem of crisp classification. There have been many different approaches to address this problem. Lofti Zadeh, an influential computer scientist, created a complexity-inspired system to transform quantitative variables into linguistic (natural language) ones, and characterized the relations between variables using conditional statements he termed fuzzy membership functions. Formulated during a period of high academic angst over the inability of control theory, mechanistic systems science or even 
the laws of thermodynamics to explain human social systems, Zadeh's (1976) proposed framework stimulated immediate interest in the quantitative disciplines, and now enjoys widespread application in the applied social sciences (especially in business intelligence-related fields such as supply chain management). SES theorists in ecology, who as demonstrated above tend to gravitate toward and communicate their worldview through heuristic models (see Figures 1 and 2 depicting the adaptive renewal cycle), tackle the issue by deconstructing concepts which are difficult to quantitatively define, or which are affected by feedback, into constituent scale levels. Importantly, SES theorists stress that the problem of ontological vagueness and complex feedback relations can be effectively addressed within a heuristic context only if there is balanced, simultaneous and holistic analysis conducted at all scale levels of the deconstructed concept (Berkes et al. 2003).

What does all this have to do with community resilience? As it turns out, both 'community' and 'resilience' are ontologically vague terms with many undefined or (quantitatively) undefinable components; put together, they signify a particularly troublesome concept to concisely, unambiguously or authoritatively define. Many 'paradigms' of definition of these terms exist in academia, and some reviewers dedicate whole articles to plotting the lineage of their usage among different disciplines (e.g. Manyena 2006, Brand and Jax 2007). Nonetheless, a standardized definition of resilience, community, or community resilience has not materialized. A palpable sense of frustration over this situation often arises in the literature: 
Despite more than three decades' worth of collective research experience on the concept, resilience still means different things to people in different fields ... The result is a confused lexicon of meanings and approaches to understanding resilience to external shock or natural hazards.

- Zou et al 2012 p.22

Looking back, one wonders if perhaps the social and psychological sciences should have created their own language, free from inherited meanings, but the term [resilience] is probably here to stay... The concept of "community resilience" raises the same concerns. - Norris et al 2008 p.128

...The definition of resilience, however, should be independent of the object of analysis and, in the interest of facilitating the formulation of compatible policy goals in both the public and private sector ... the same definition should be used in all decisionmaking processes. Establishing a uniform definition is critically important. - Argonne National Laboratory 2012 p. $11^{15}$

The difficulties arise when, little by little, the polysemy [of resilience] seems to legitimize a semantic blur that creates theoretical and operational dead ends. In view of occasional contrary injunctions, the concept ends up being "inoperative", being reduced to some sort of unattainable discursive utopia to the point where some researchers have considered the concept too vague to be used in order to prevent disaster. Reghezza-Zitt et al 2012, 'What Resilience is Not: Uses and Abuses', p.1

Even though a completely satisfactory solution to the ontology problem is not provided by either fuzzy sets or scalar heuristics (Markusen 1999; Wilson 2012), some type of conceptual abstraction is probably necessary to reduce the complexities of concepts such as communities and community resilience, especially to facilitate research which attempts to measure these concepts (Tate 2012). A tentative parallel can also be drawn between the additive index and the heuristic approach of SES, in that

\footnotetext{
${ }^{15}$ After chiding academia for not coming up with an authoritative definition, and after an impressively broad review of definitions in the literature, Argonne scientists come up with a rather underwhelming official one: "The ability of an entity - asset, organization, community, region - to anticipate, resist, absorb, respond to, adapt to, and recover from a disturbance." Policymakers may still wonder if the referenced 'ability' is an inherent attribute or the result of a process. They may also take 'disturbance' to mean singular, fast-onset extreme events, and ignore slower-onset disturbance mechanisms in policy decisions.
} 
they both have the effect of deconstructing the larger concept of community resilience into smaller components which can be analyzed by themselves or deconstructed to further levels. The main difference is that geographers often deconstruct resilience into categorical dimensions (Social, Economic, Institutional, etc.), whereas SES ecologists deconstruct it into scalar ones (micro/meso/macro, or individual/ community/ ecosystem, etc.). Thus, an opportunity may exist for better integrating the human dimensions of resilience while better accounting of the multiple levels at which resilient characteristics and processes operate. This is a needed improvement, as the recommended balancing of multi-scale resilience indicators is currently lacking in geography. Current geographic studies which, as mentioned, tend to operationalize Cutter et al.'s DROP (2008) or SOVI (2003) conceptual models, disproportionately rely on individual and household-level variables gleaned from Census data. Figure 6 shows an example from Cutter et al.'s (2010) highly cited additive index of resilience indicators based on the DROP model; highlighted are variables judged to represent individual or household-level qualities (19 out of 36 variables). It should be noted that eight of the remaining variables do reflect more meso-level, community-scale characteristics; but these focus on structural and institutional indicators (e.g. the 'Evacuation Potential' indicator of 'principles arterial miles per community' and the 'Social Capital - Advocacy' indicator of 'number of social advocacy organizations per 10,000 population). Less attention is paid to larger-scale, government-initiated processes, or the locally-felt benefits of large-scale business activities which may boost the level of resilience of several communities. Least represented of all are community-level variables actually 
indicating collective, self-organized action undertaken by and for community inhabitants

in response to the perceived specific environmental hazards and threats. ${ }^{16}$ Though it may necessitate sourcing and integrating a diverse set of place-based indicator data, a more balanced and multi-scalar approach to variable selection would enhance applied community resilience studies in geography.

\footnotetext{
${ }^{16}$ These are probably the least-represented because they are the most place-dependent. They arise from the historic, cultural, and every other human context of specific communities, are applied to counter place-specific environmental hazards, and exhibit wide spatial variability between communities in different contexts. Consequently, they are the least likely to 'scale up' to larger-area levels of resilience analysis, such as this example (Cutter 2010) which characterized the community resilience of counties in the Southeastern U.S. The authors of the index ran into the same problem when considering how to include the 'Ecological' dimension of resilience as recommended in the DROP model (with candidate variables including wetland acreage, percent impervious land cover, biodiversity, erosion rates, number of coastal defense structures, etc.). In the end, the authors did not include an 'Ecological' dimension, stating that the wide physical variability of the large study area made many ecological indicators inapplicable (Cutter 2010). More problems associated with large-scale resilience studies are explored in the next section.
} 
Figure 6. Community resilience indicators utilized by Cutter (2010)

\begin{tabular}{|c|c|c|c|c|c|}
\hline Category & Variable & $\begin{array}{l}\text { Effect on } \\
\text { Resilience }\end{array}$ & Justification & Data Source & Scale of Indicator \\
\hline $\begin{array}{l}\text { Social Resilience } \\
\text { Educational } \\
\text { equity }\end{array}$ & $\begin{array}{l}\text { Ratio of the pct. population with } \\
\text { college education to be pet. } \\
\text { population with no high scbool } \\
\text { diploma }\end{array}$ & Negative & $\begin{array}{l}\text { Noms et al. } 2008 \\
\text { Morrow } 2008\end{array}$ & US. Census 2000 & Indiv./HH \\
\hline Age & Percent non-elderly population & Positive & Morrow 2008 & U.S. Census 2000 & Indiv./HH \\
\hline $\begin{array}{l}\text { Transportation } \\
\text { access }\end{array}$ & \begin{tabular}{|l|} 
Percent population with a vehicle \\
\end{tabular} & Positive & Tierney 2009 & U.S. Census 2000 & Indiv./HH \\
\hline $\begin{array}{l}\text { Communication } \\
\text { capacity }\end{array}$ & \begin{tabular}{|l|} 
Percent population with a telephone \\
\end{tabular} & Positive & Colten et al. 2008 & U.S. Census 2000 & Indiv./HH \\
\hline $\begin{array}{l}\text { Language } \\
\text { competeacy }\end{array}$ & $\begin{array}{l}\text { Percent population not speaking } \\
\text { English as a second language }\end{array}$ & Positive & Morrow 2008 & U.S. Ceasus 2000 & Indiv./HH \\
\hline Special needs & $\begin{array}{l}\text { Perceat population without a } \\
\text { sensory. physical, or mental } \\
\text { disability }\end{array}$ & Positive & Heinz Center 2002 & U.S. Census 2000 & Indiv./HH \\
\hline Health coverage & $\begin{array}{l}\text { Percent population with bealth } \\
\text { insurance coverage }\end{array}$ & Positive & Heinz Center 2002 & US. Census 2000 & Indiv./HH \\
\hline \multirow{2}{*}{$\begin{array}{l}\text { Ecomomic Resilie } \\
\text { Housing capital }\end{array}$} & $\begin{array}{l}\text { nce } \\
\text { Percent bomeownership }\end{array}$ & Positive & Norns et al. 2008 & US. Census 2000 & \\
\hline & $\begin{array}{l}\text { Percent employed } \\
\end{array}$ & Positive & $\begin{array}{l}\text { Cutter et al. } 2008 \mathrm{a} \\
\text { Themey es a. 2001 }\end{array}$ & U.S. Census 2000 & $\begin{array}{l}\text { Indiv./HH } \\
\text { Indiv./HH }\end{array}$ \\
\hline $\begin{array}{l}\text { Ficome and } \\
\text { equality }\end{array}$ & GDI coefficient & Positive & Nornis et al. 2008 & $\begin{array}{l}\text { Computed from } \\
\text { US. Census } 2000\end{array}$ & Community \\
\hline $\begin{array}{l}\text { Single sector } \\
\text { employment } \\
\text { dependence }\end{array}$ & $\begin{array}{l}\text { Percent population not employed in } \\
\text { fanming, fishing, forestry, and } \\
\text { extractuve industries }\end{array}$ & Positive & $\begin{array}{l}\text { Berke \& } \\
\text { Campanella } 2006 \\
\text { Adger } 2000\end{array}$ & US. Census 2000 & Indiv./HH \\
\hline Employment & $\begin{array}{l}\text { Percent female labor force } \\
\text { participation }\end{array}$ & Positive & NRC 2006 & U.S. Census 2000 & Indiv./HH \\
\hline Business size & Ratio of large to small businesses & Positive & Nornis et al. 2008 & $\begin{array}{l}\text { County Business } \\
\text { Patterns (NAICS) } \\
2006\end{array}$ & Institutional \\
\hline Health Access & $\begin{array}{l}\text { Number of physicians per } 10.000 \\
\text { population }\end{array}$ & Positive & Norris et al. 2008 & US. Census 2000 & Indiv./HH \\
\hline \multicolumn{2}{|c|}{ Instifutional Resilience } & & & & \\
\hline Mitigation & $\begin{array}{l}\text { Percent population covered by a } \\
\text { recent hazard mitigation plan }\end{array}$ & Positive & $\begin{array}{l}\text { Burby et al. } 2000 \\
\text { Godschalk } 2007 \\
\end{array}$ & FEMA.gov & Indiv./HH \\
\hline Flood coverage & $\begin{array}{l}\text { Percent housing units covered by } \\
\text { NFIP policies }\end{array}$ & Positive & Burby et al. 2000 & bsa.nfipstat com & Indiv./HH \\
\hline $\begin{array}{l}\text { Municipal } \\
\text { services }\end{array}$ & $\begin{array}{l}\text { Percent musicipal expendinures for } \\
\text { fire. police. and EMS }\end{array}$ & Positive & Sylves 2007 & $\begin{array}{l}\text { USA Counties } \\
2000\end{array}$ & Government \\
\hline Marigation & $\begin{array}{l}\text { Perceat population participating in } \\
\text { Community Rating System for Flood } \\
\text { (CRS) }\end{array}$ & Positive & Godshall 2003 & FEMA EOV & Indiv./HH \\
\hline $\begin{array}{l}\text { Political } \\
\text { fragmentation }\end{array}$ & $\begin{array}{l}\text { Number of governments and special } \\
\text { districts }\end{array}$ & Negative & Noms et al. 2008 & US. Census 2002 & Government \\
\hline $\begin{array}{l}\text { Previous } \\
\text { disaster } \\
\text { experience }\end{array}$ & Number of paid disaster declarations & Positive & $\begin{array}{l}\text { Cunter et al } \\
2008 \mathrm{a}\end{array}$ & FEMA.gov & Government \\
\hline $\begin{array}{l}\text { Mintigation and } \\
\text { social } \\
\text { conpectivity }\end{array}$ & $\begin{array}{l}\text { Percent population covered by } \\
\text { Citizen Corps programs }\end{array}$ & Positive & Godesalk 2003 & citizen.cosps.gov & Community \\
\hline Mitigation & $\begin{array}{l}\text { Perceat population in Storm Ready } \\
\text { commmunities }\end{array}$ & Positive & Godshalk 2003 & $\begin{array}{l}\text { stormiready noas. } \\
\text { gov }\end{array}$ & Community \\
\hline $\begin{array}{l}\text { Infressuctare Re } \\
\text { Housing type }\end{array}$ & $\begin{array}{l}\text { silience } \\
\text { Percent housing units that are not } \\
\text { mobile bomes }\end{array}$ & Positive & Cutter et al. 2003 & U.S. Census 2000 & Indiv./HH \\
\hline $\begin{array}{l}\text { Sbeliter capacity } \\
\text { Medical } \\
\text { capacity }\end{array}$ & $\begin{array}{l}\text { Percent vacant rental units } \\
\text { Number of bospital beds per } 10,000 \\
\text { population }\end{array}$ & $\begin{array}{l}\text { Positive } \\
\text { Positive }\end{array}$ & $\begin{array}{l}\text { Therney } 2009 \\
\text { Auf de Heide and } \\
\text { Scanloz } 2007\end{array}$ & $\begin{array}{l}\text { US. Census } 2000 \\
\text { Amerencan Hospital } \\
\text { Directory } \\
\text { wwww.abd.com }\end{array}$ & $\begin{array}{l}\text { Indiv./HH } \\
\text { Institutional }\end{array}$ \\
\hline $\begin{array}{l}\text { Access/ } \\
\text { evacuation } \\
\text { potential }\end{array}$ & $\begin{array}{l}\text { Pribciple arterial miles per square } \\
\text { mile }\end{array}$ & Positive & NRC 2006 & $\begin{array}{l}\text { Gis denved from } \\
\text { National Atlas.gov }\end{array}$ & Community \\
\hline Housing age & $\begin{array}{l}\text { Perceat bousing units not bullt } \\
\text { before } 1970 \text { and after } 1994 \\
\end{array}$ & Positive & Mileti 1999 & $\begin{array}{l}\text { Ciry and Coumty } \\
\text { Databook } 2007\end{array}$ & Community \\
\hline Sbeltering Deeds & $\begin{array}{l}\text { Number of hotels/motels per square } \\
\text { mile }\end{array}$ & Positive & Tiemey 2009 & $\begin{array}{l}\text { County Business } \\
\text { Patterns (NAICS) } \\
2006\end{array}$ & Institutional \\
\hline Recovery & \begin{tabular}{|l|} 
Number of public schools per square \\
mile
\end{tabular} & Positive & $\begin{array}{l}\text { Rogan and } \\
\text { Johnston } 2005\end{array}$ & Gnis.usgs.gov & Institutional \\
\hline \multicolumn{2}{|c|}{ Communiny Copital } & & & & \\
\hline $\begin{array}{l}\text { Place } \\
\text { attachment }\end{array}$ & Net intermational migration & Negative & Morrow 2008 & ceasus.gov & Community \\
\hline $\begin{array}{l}\text { Place } \\
\text { attachmeat }\end{array}$ & $\begin{array}{l}\text { Percent population borm in a state } \\
\text { that still resides in that state }\end{array}$ & Positive & $\begin{array}{l}\text { Vale \& } \\
\text { Campanella } 2005\end{array}$ & US. Census 2000 & Indiv./HH \\
\hline $\begin{array}{l}\text { Political } \\
\text { engagement }\end{array}$ & \begin{tabular}{|l|} 
Percent voter participation in the \\
2004 election
\end{tabular} & Positive & Morrow 2008 & $\begin{array}{l}\text { Ciry and County } \\
\text { Databook } 2007\end{array}$ & Indiv./HH \\
\hline $\begin{array}{l}\text { Social capital- } \\
\text { relipion }\end{array}$ & $\begin{array}{l}\text { Number of religious adhereats per } \\
10.000 \text { population }\end{array}$ & Positive & $\begin{array}{l}\text { Morrow } 2008 \\
\text { Murpby } 2007\end{array}$ & $\begin{array}{l}\text { Asso. of Religion } \\
\text { Data Archives }\end{array}$ & Community \\
\hline $\begin{array}{l}\text { Social capital - } \\
\text { civic } \\
\text { involvement }\end{array}$ & $\begin{array}{l}\text { Number of civic organizations per } \\
\text { 10.000 population }\end{array}$ & Positive & $\begin{array}{l}\text { Morrow } 2008 \\
\text { Murply } 2007\end{array}$ & $\begin{array}{l}\text { County Business } \\
\text { Patters s (NAICS) } \\
2006\end{array}$ & Institutional \\
\hline $\begin{array}{l}\text { Social capital - } \\
\text { advocacy }\end{array}$ & $\begin{array}{l}\text { Number of social advocacy } \\
\text { organizations per } 10,000 \text { population }\end{array}$ & Positive & Murphy 2007 & $\begin{array}{l}\text { County Business } \\
\text { Patterns (NAICS) } \\
2006\end{array}$ & Institutional \\
\hline Innovation & $\begin{array}{l}\text { Percent population employed in } \\
\text { creative class occupations }\end{array}$ & Positive & Norris et al. 2008 & $\begin{array}{l}\text { USDA Economic } \\
\text { Research Service } \\
\text { ers usda gov }\end{array}$ & Community \\
\hline
\end{tabular}

36 Total Indicators: Individual/Household: 19 (53\%) Government/Institutional: 9 (25\%) Community: 8 (22\%) 


\subsection{The problem of scale}

What is the proper scale of analysis for an assessment of community resilience? Judging from the wide-ranging literature on disaster resilience, there is little agreement on this issue. Every areal unit of analysis from a country down to city Census blocks has been used as the geographic unit of analysis in research on community-level resilience (Godschalk 2003; Chan and Wong 2007, Norris et al. 2007; Ebert et al. 2009; Downes et al. 2013). A typical approach is to characterize community disaster resilience at the regional level, spatially aggregating study variables into counties or U.S. Census metropolitan statistical area (MSA) geographies (an example of which is highlighted in Figure 7). Without much rationale for this choice of areal unit of analysis, the output of many studies is simply maps purporting to show the level of community disaster resilience over relatively large areas. While it may be difficult to pinpoint the rationale behind these assumptions, there should be little doubt that the outcome is important for stakeholders in emergency management and disaster preparedness who look to academia for advice on the best way to confront the issue of community disaster resilience (Cumming et al. 2006). Either the spatial scale chosen to characterize community provides an acceptable level of actionable information or it does not. Likewise, either the chosen scale reflects the current and probable future scale of community resilience intervention, or it does not. As previously noted, there has been a change of tone of federal-level emergency management policy and a shift of focus from social vulnerability to community resilience. It is also observed that researchers and professionals outside of geography who publish articles on the subject of community 
Figure 7. Example of community resilience research with large, heterogenous study units

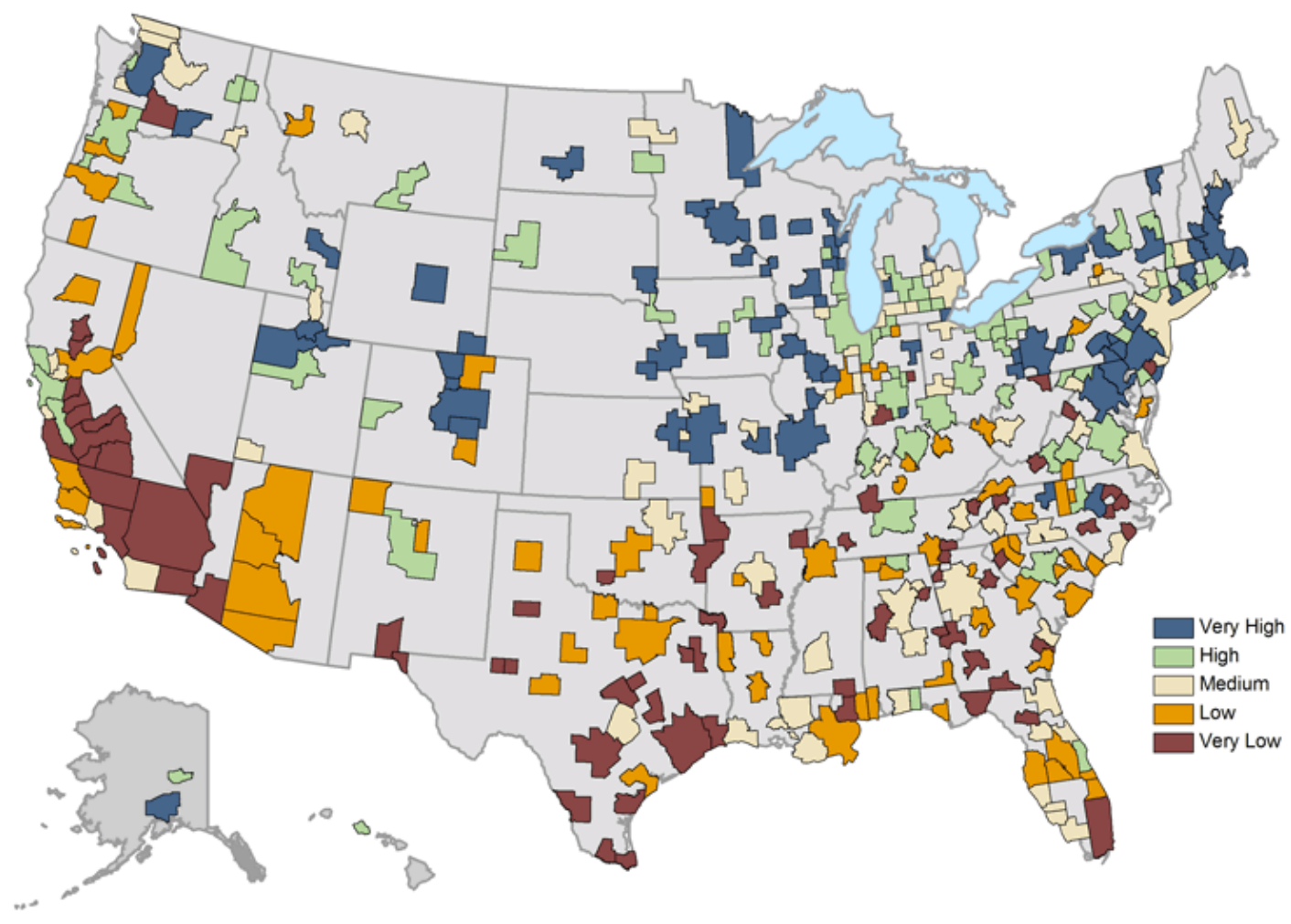

This map of nation-wide community resilience at the Census Metropolitan Statistical Area (MSA) level, based on the Resilience Capacity Index (RCI) conceptual framework (UC Berkley 2013), utilizes a composite index of 'Socio-Demographic', 'Community Connectivity' and 'Regional Economic' indicators. The map portrays the St. Louis MO-IL MSA (consisting of St. Louis City, St. Charles, Jefferson and St. Louis counties in Missouri, as well as Jersey, Calhoun, Macoupin, Madison, St. Clair and Monroe counties in Illinois) as having a 'Very High' level of community resilience. This portrayal, and derivative interpretations of the map, suffer greatly from the ecological effect, a type of mathematical fallacy in which aggregate-level statistics cannot be used to characterize lower-level components. An analytic conclusion that communities in St. Louis City, MO and East Saint Louis, IL, being part of the larger MSA, are also very highly resilience is untenable both logically and in point of fact. Likewise, a conclusion that most communities in southern California exhibit very low levels of resilience would be equally unsound. Though most geographers would probably agree this approach conceals and mischaracterizes the spatial variability of indicators within the St. Louis MSA, large-area aggregation of resilience indicators is still common practice in the discipline. Not only is there risk that misinformation associated with the spatial aggregation could attenuate into the policy decisions of state and federal-level emergency managers, the scale of analysis does not match the local-level and place-specific qualities of community resilience. In the worst case, the map may lead policy makers to ignore areas with the highest need; in the best case, it probably doesn't tell policy makers anything they don't already know. 
disaster resilience are increasingly choosing a local spatial scale in their studies. Downes et al. (2013) systematically examines the spatial scales of 106 recent community resilience studies spanning 35 social science disciplines, finding that $46.6 \%$ were conducted at the local scale, $21.9 \%$ regional, $26.0 \%$ national/continental, and $5.5 \%$ at a global scale. This thesis' examination of recent community vulnerability/ resilience studies outside of geography (e.g. in public health, emergency management, civil engineering and regional planning, etc.) concurs with this assessment (c.f. Morrow 1999, Kulig 2000, Kendra and Wachtendorf 2007, Wolf et al. 2010, Geis 2000, Hall and Zautra 2010, Jabeen et al. 2010, Flanagan et al. 2011, Boon et al. 2012, Teo et al. 2013, Cohen et al. 2013, Chandra et al. 2013). This pattern lends credence to the argument that the future scale of community resilience intervention will be local and the dimensions of resilience studied will be place-specific.

A more a fundamental justification against large-scale community resilience studies can be found in early work in physics. Nearly a century ago, the field of statistical physics provided the mathematical argument against large-scale characterization of local-scale phenomena. This occurred as an important early discovery from the tradition of physical systems science, in the observation that system component variables tend to display increasing levels of random interaction as one examines the system at smaller scales, which may lead to wide local variations in both the attributes of variables and the relationships between variables. For example, while a global pattern of energy balance may exist where incoming radiation from the sun is equaled by emittance from the planet, there are extreme local variations on energy budget. 
From this, climatologists know better than to infer the state of the global energy budget by studying data from just a few sites; conversely, they would not attempt to infer the characteristics of specific locations using only data aggregated at large spatial scales. By the 1950's, this latter concept was codified as the 'ecological effect' to the statistical analysis of data in the social sciences (most notably by Robinson 1950 and Durkheim 1951). The essence of the ecological effect is that characteristics of individuals, or individual locations, cannot be inferred from aggregated data, and that correlations between variables can be different depending on the scale at which they are analyzed (Subramanian et al. 2009). Practical examples of this effect and the dangers of making assumptions based on it abound in the social and natural sciences literature (Blakely and Woodward (2000) provides illustrations in epidemiological research). For example, while the sum total of economic indicators in a given country may point to a net gain in standards of living, there probably exist pockets of quite substandard living conditions, as well as superior conditions and anywhere in between. In this situation, if one is tasked with reporting on the state of community standard of living for a particular location, it would seem unwise to use data aggregated to large-area spatial units, owing to the inherent variability of individual communities within each unit. Unfortunately, as evidenced by Figure 7 above, these methods are currently employed in some geographic studied which measure community disaster resilience (see also the scale chosen in Borden et al. 2007, Cutter et al. 2010). Based on the above findings, a paradigmatic shift toward the study of specific small-scale localities is needed to bring 
geographic studies of community disaster resilience in line with the current and future information needs of emergency managers.

Neither an abandonment of the additive index as an analytic too ${ }^{17}$, nor a wholesale embrace of complexity science is recommended here. When community resilience analyses are produced and handed to emergency managers, simple aggregated indices of readily available socioeconomic data can in fact lead to saved lives and property during a disaster. Likewise, an analysis of processes that lead to community resilience based purely on current understandings of complexity science would almost certainly fall short of the emergency management community's expectation of clear, actionable information - in the worst case, it could actually lead to inaction (Markusen 1999). What is recommended is a balanced approach that attempts to integrate key findings and methods from the wide range of disciplines discussed in this review, thus better aligning with the current geographic ideals of transdisciplinary linkage, methodological pluralism and multi-perspective reflexivity. The aim is to provide emergency managers a more holistic understanding of issues such as disaster vulnerability and resilience, thereby reducing the uncertainty in the question of which communities are more or less resilient; an uncertainty which may have heretofore been elevated by geography's adherence to methods derived from a linear-oriented conceptual framework.

\footnotetext{
${ }^{17}$ In fact, a modified weighted index to measure disaster resilience for St. Louis City neighborhoods will be used in this thesis, but will employ methods to characterize the complex influence among variables and to integrate local expert knowledge into the variable weighting procedure.
} 


\section{CHAPTER 3. METHODOLOGY}

In view of the above findings and recommendations, a new approach to measuring community disaster resilience is proposed. The utility of the developed approach is then demonstrated with an analysis of community resilience conducted at the neighborhood level in the City of St. Louis, Missouri. The methods outlined here represent a novel attempt at operationalizing concepts from complexity science and non-linear systems theory toward measuring disaster resilience at the community level within an urban context. ${ }^{18}$ More importantly, the chosen approach is intended to adhere more explicitly to promote the geographic objectives of transdisciplinary linkage, methodological pluralism, and multi-perspective reflexivity.

This thesis advocates a place-specific framing of community resilience studies, both in terms of scale as well as in indicator and variable development. It also proposes that some measurement tools within complexity science are applicable to the measurement of community resilience. While the selection of methods here reflects this stance, they are by no means meant as normative, nor can they be assumed to scale up to broader levels of analysis or be applied to other places without significant and thoughtful modification. Measuring community resilience to disaster, perhaps more so

\footnotetext{
${ }^{18}$ Current research in the discipline shows an emergent similarity to the complexity paradigm but does not self-identify as such and applies some methods seen as ill-advised by complexity science. Rufat (2013), for example, distrusts the simple additive matrix, but also relies solely on the internal statistics of his data to uncover clusters of vulnerable groups in a specific place. He was left to explain the drivers of vulnerability as 'the machine' defined them. This application of remote sensing / spectral clustering analysis methods should be encouraged, as it is clearly in the interests of methodological pluralism. But it should be pointed out that unsupervised classification assumes no a priori knowledge of the study area and trades this off for a high error potential. Rufat could have used methods which exploited his deep expert knowledge of the study area (Lyon, France).
} 
than other qualities of human systems, requires methods that reflect the uniqueness of the place and population studied.

\subsection{Environmental Hazard Profile}

The all-hazards approach to disaster mitigation stressed in recent research (c.f. Cutter 2003) remains highly relevant and important, even though the scale of some geographic studies precludes incorporation of place-specific environmental hazard information. Therefore, in the context of small-scale resilience studies, it is proposed here that a framework for the identification and characterization of environmental hazards affecting a community of interest is included in all such resilience studies. Luckily (and somewhat ironically), the United States federal government already utilizes a good template for such place-based all-hazards profiles. The Federal Emergency Management Agency (FEMA) has for decades provided incentives for local government to conduct research on hazards in their jurisdictions and develop disaster mitigation plans. For example, FEMA requires a written all-hazards mitigation plan for cities and school districts applying for federal disaster mitigation grants and other assistance programs. The required content for these plans, codified in the Stafford Act (42 U.S.C. 5165) and FEMA regulation 44 CFR 201, include: Community/County Profile (including history, geography, demographics, governmental jurisdiction, etc.), Risk Assessment (identifying and characterizing all environmental hazards known to exist within the jurisdiction), City/County Capability Assessment (including health and public safety infrastructure, emergency response capabilities, sheltering capacity, critical 
infrastructure assessment, etc.) and Mitigation Plan (identifying hazard mitigation goals, objectives and strategy, key stakeholders, inter-agency coordination plans, etc.). Such plans, where they exist, can provide important insight into the characteristics of environmental hazards affecting certain cities, local leaders' and residents' perception of those hazards, and the priorities for mitigation identified by the local government. With this in mind, it is recommended here that, when available, all-hazard mitigation plans submitted to FEMA by local jurisdictions are utilized in urban community resilience studies for the purpose of producing a place-specific, locally sourced all-hazards profile. Additionally, the comparative hazard assessment and perception information contained in the mitigation plan, constituting a set of expert rankings, can be directly utilized to set weights for environmental hazard variables in a vulnerability index. This practice would be especially helpful in instances where the direct elicitation of expert rankings in surveys or interviews proves impractical.

\subsection{Combined Environmental and Socioeconomic Vulnerability Index}

Though most community resilience paradigms now focus more on assets and strengths as opposed to deficits or weaknesses (which are generally framed in terms of adverse socioeconomic conditions), the vulnerability which these deficiencies can create inside communities should not be ignored. In particular, following decades of work by critical social theorists demonstrating that disadvantageous socioeconomic conditions amount to hazards, geographers are rightly hesitant to cease accounting for social vulnerabilities, especially in the urban context (Rufat 2103). Therefore, it is 
recommended that a vulnerability analysis phase be included in urban community resilience studies, to combine environmental and socioeconomic hazards. Place-specific environmental hazards data should preferably be gleaned from local expert knowledge sources, as in the FEMA hazard mitigation plans referenced above. For socioeconomic indicators and representative variables of vulnerability, a plethora of data sources exists (King 2001, Flax et al. 2002, Cutter et al. 2003, Adger et al. 2004, Cardona 2005). However, the selection of socioeconomic indicators and variable data should also take advantage of the reporting of local experts, such municipal departments of health or public safety, which often reveals the unique stressors and challenges faced by communities in the area studied.

\subsection{Resilience Index}

An apparent, yet unexplained, methodological inconsistency in the shift of focus from vulnerability to resilience in community disaster research is that the same indicators and variables used to populate vulnerability indices are also commonly used to populate resilience indices (see, for example, the variables utilized by Cutter et al. 2003 and Cutter et al. 2008). Given that the components of resilience are not typically identical to (or opposites of) the components of vulnerability, a new system for constructing separate vulnerability and resilience indices and apportioning indicators into each is proposed. The principle followed for apportioning indicators is: if a certain variable can be viewed as more of a deficiency, or as a process working against a populations' ability to cope with extreme events, it will be included in the vulnerability 
index. If the variable can be viewed more as a quality or process (especially an endogenous, emergent process or intentional/collective action) which increases community capacity to cope with extreme events, it will be included in the resilience index. This dual vulnerability-resilience approach has advantages beyond that of adhering to multidisciplinary recommendations for separating these concepts in the analysis phase. It will also allow both metrics, once populated with variables and parameterized, to be juxtaposed to characterize the relative agreement between the degree of risk a community faces and the amount of resilience capacity put into play. Therefore, a study conducted by this method can provide a characterization of both absolute resilience (in a map depicting resilience 'scores' alone) and, more importantly, relative resilience (rendered as a weighted suitability surface).

Also proposed in this thesis is a resilience index structure that divides indicators into the sub-scales at which they can be perceived to operate: the micro (individual / household), meso (neighborhood), and macro (government/institutional) levels. This practice not only enables the balancing of multi-scalar resilience components as recommended in the literature, but also permits emergency managers and community leaders to analyze study outputs, such as thematic maps, according to their organizational focus and/or level of jurisdiction. Indicators chosen to populate each sub-level of the multi-scale resilience index can be sourced from a variety of studies that have examined the dimensions of community resilience, including those focused on business management (Bhamra et al. 2011), environmental science (Bahadur et al. 2013), public health (Cohen et al. 2013), social psychology (Windle 2011), econometrics 
(Chan and Wong 2007), geography (Renschler et al. 2010) as well as others. As in vulnerability indices, variables chosen to represent resilience indicators should whenever possible include place-specific data sourced from local expert reporting.

\subsection{Determining Indicator Weights}

Weighting of indicators in vulnerability and resilience indices has received a variety of treatments in the literature, from equal-weighting schemes, to knowledgebased ranking of individual indicators, to weighting schemes based on internal multivariate statistics of indicators (Ratick and Osleeb 2011). However, Cutter et al. (2010) argues for standardization of equal indicator weighting given that method's transparency and ease of understanding may be untenable due to the potential for knowledge-based rankings to unveil indicator importance or preference for management purposes. At the local scale at which this resilience study is performed, and considering the level of enthusiastic stakeholder buy-in which such studies can elicit, this potential is considered to be high. Therefore, a method for integrating local expert knowledge and lay opinions of vulnerability and resilience indicator importance, for the purpose of defining indicator weights, is warranted. The knowledge-based approach has advantages over statistical methods in that perceived relations are taken into account, whereas multivariate statistical methods are only able to evaluate the relative importance between indicators inherent in the analyzed data (Nardo et al. 2005a). Also, relational statistics, especially when applied to variables in human systems, are at risk to spurious correlations (Hiete et al. 2011). 
The analytic hierarchy process (AHP) can be extended to set weights for individual indicators in both vulnerability and resilience indices based on qualitative pair-wise comparison of indicators in a digraph. AHP is a robust decision support tool originally developed within industrial engineering for using expert ratings to select preferred means from a set of alternatives to achieve a normative production outcome (Saaty 2008). Here, the AHP approach can be used to define the relative importance of study indicators within an index; however, in this case the purpose is not to identify and select out the most dangerous threat in the vulnerability index or most important protection in the resilience index, but to retain the information regarding relative indicator importance for the purpose of defining weights. In this context, the 'aggregation of individual judgments' approach to AHP (Aczel and Saaty 1983, Wu et al. 2010) is operationalized as follows:

A pairwise indicator comparison matrix (or a set of matrices if the there are two or more sub-levels in the index to be weighted) is constructed. Indicators within their respective sub-scale are assessed against each other as to their importance or relative contribution to resilience within their respective subscale. The scale of quantified judgment of comparative importance can be set from 1.0-9.0 according to Table 3.

Table 3. Quantitative Scale for Indicator Comparisons (Wu et al. 2010)

\begin{tabular}{lc} 
Verbal Judgment of i-j Comparison & Numerical Rating \\
\hline Of Overwhelming Importance & 9 \\
Much More Important & 8 \\
Of Moderately More Importance & 7 \\
Slightly More Important & 6 \\
Of Equal Importance & 5
\end{tabular}


Slightly Less Important $\quad 4$

Of Moderately Less Importance 3

Much Less Important 2

Insignificant by Comparison $\quad 1$

Given a set of indicators indexed $i$ and $j$, let $C_{1}, C_{2}, \ldots, C_{n}$ represent the set of indicators in a certain sub-index (such as the Socioeconomic sub-index of the vulnerability index) and $a_{i j}$ represent a quantified judgment on a pair of indicators $C_{i}$ and $C_{j}$. The pairwise comparison matrix with $n$ indicators is presented below, where $a_{12}$, for instance, means the quantified judgment between $C_{1}$ on the first row and $C_{2}$ on the second column:

$$
A=\left[a_{\mathrm{ij}}\right]=\left[\begin{array}{cccc}
1 & a_{12} & \ldots & a_{1 \mathrm{n}} \\
a_{21} & 1 & \ldots & a_{2 \mathrm{n}} \\
\vdots & \vdots & \ddots & \vdots \\
a_{\mathrm{n} 1} & a_{\mathrm{n} 2} & \ldots & a_{\mathrm{nmax}}
\end{array}\right]
$$

To compute the importance for each indicator in terms of its comparative contribution to the overall goal, AHP consists of three steps. First, values in each column of the pairwise comparison matrices are summed. Second, the number of quantified judgments $\mathrm{a}_{\mathrm{ij}}$ in the pairwise comparison matrix is divided by its column total, which results in the normalized pairwise comparison matrix. Third, the average of the elements in each row of the normalized pairwise comparison matrix is calculated. As a result, these averages represent the weights of the indicators.

As a validation measure, AHP uses a consistency ratio to evaluate the consistency of the pairwise judgments (Zahedi 1986). The first step is to use the pairwise comparison matrix as shown in Eq. 1 to multiply the weights of the indicators, specified as an $n \times 1$ matrix, computed in the third step of the above procedure. This 
results in a weighted sum vector for each indicator. Next, the weighted sum vectors are divided by the corresponding weights for each indicator. Third, the average of these values, denoted as $\lambda_{\max }$, is calculated. The consistency index $(\mathrm{Cl})$ is then computed by:

$$
C I=\left(\lambda_{\max }-n\right) /(n-1)
$$

where $n$ is the number of indicators. Finally, the consistency ratio (CR) is computed as $\mathrm{CR}=\mathrm{Cl} / \mathrm{RI}$, where $\mathrm{RI}$ is the random index of a randomly generated pairwise comparison matrix. Table 4 provides RI values with different $n$. When CR is less than 0.10 , the matrix is considered to be consistent.

Table 4. RI Random Index Values for Various $n$

\begin{tabular}{lllllll}
\hline $\mathrm{n}$ & 3 & 4 & 5 & 6 & 7 & 8 \\
\hline $\mathrm{RI}$ & 0.58 & 0.90 & 1.12 & 1.24 & 1.32 & 1.41 \\
\hline
\end{tabular}

\subsection{Combining Vulnerability and Resilience Indices}

When the separate vulnerability and resilience indices are calculated, there results two separate spectrums of scores for vulnerability per community and resilience per community. Since each index consists of different numbers of variables with different weighting schemes (yet are expected to be normally distributed), it makes sense to analyze these datasets using defined standard deviations as a common scale. For example, in the sample distribution datasets in Figure 8, both vulnerability and resilience datasets are normally distributed with fewer extreme scores. 
Figure 8. Sample Vulnerability and Resilience Index Score Distributions and Statistics
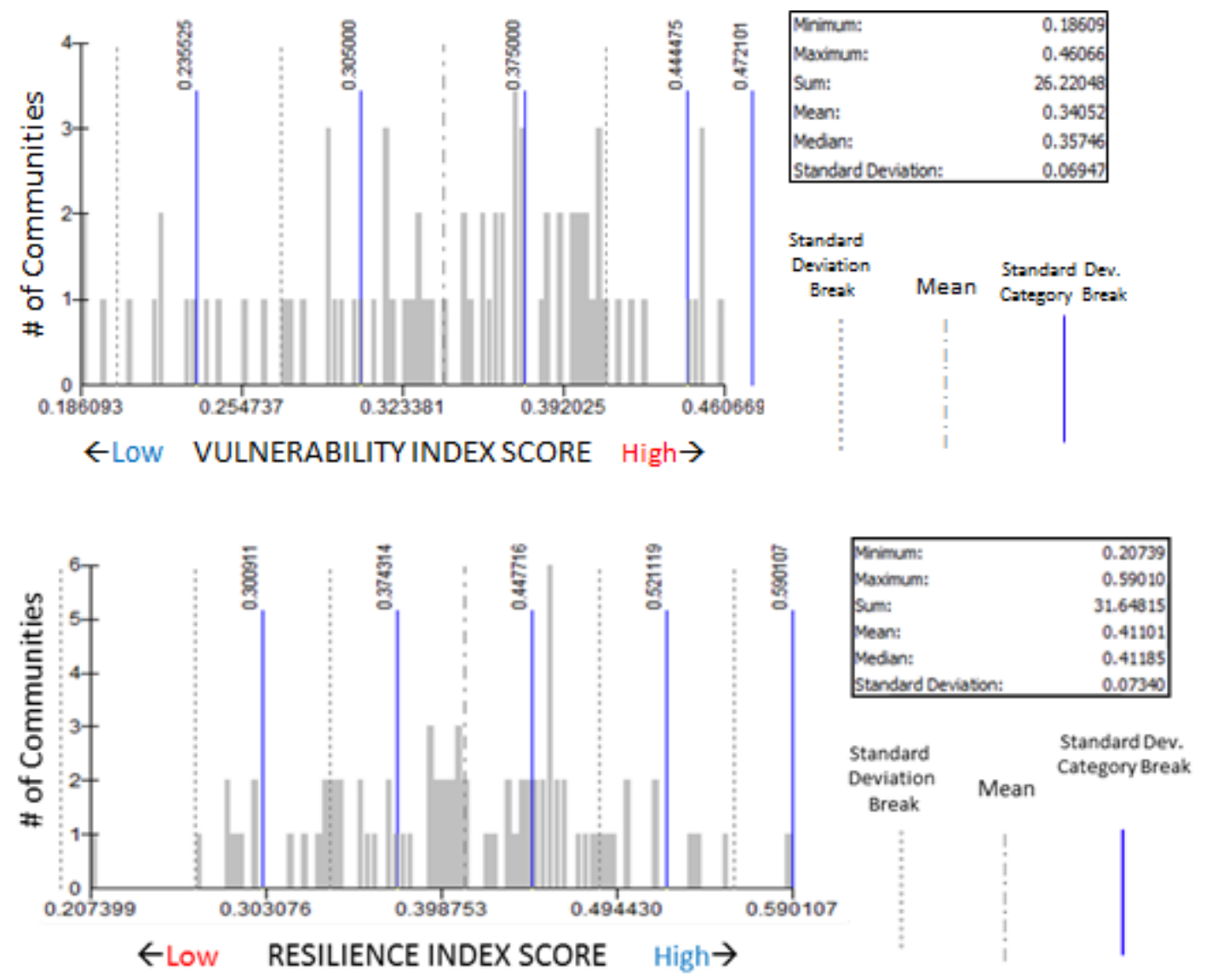

In this case, defining categories of community index scores based on standard deviations around the mean of the datasets can produce categories to facilitate comparison and combination of the two datasets. ${ }^{19}$ If categories of 'Very High', 'High', 'Medium', 'Low' and 'Very Low' are chosen, the datasets can be classified in a confusion matrix as shown in Table 5, with the output being final relative resilience categories.

\footnotetext{
${ }^{19}$ For example: 'Very Low'= Less Than -1.5 Std.Dev; 'Low'= Between -1.5 and -.5 Std.Dev; 'Medium'= Between -.5 and .5 Std.Dev; 'High'= Between .5 and 1.5 Std.Dev; 'Very High'= More Than 1.5 Std.Dev
} 
Table 5. Sample Confusion Matrix to Convert Vulnerability and Resilience Categories to Final Relative Risk Categories

\begin{tabular}{|c|c|c|c|c|c|c|}
\hline & \multicolumn{5}{|c|}{ RESILIENCE } \\
\hline & & Very Low & Low & Medium & High & Very High \\
\hline \multirow{5}{*}{ 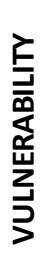 } & Very High & Critically High Risk & Very High Risk & High Risk & Medium risk & Medium Risk \\
\hline & High & Very High Risk & High Risk & Medium Risk & Medium risk & Low Risk \\
\hline & Medium & High Risk & Medium Risk & Medium Risk & Medium risk & Low Risk \\
\hline & Low & Medium Risk & Medium Risk & Medium Risk & Low Risk & Very Low Risk \\
\hline & Very Low & Medium Risk & Medium Risk & Low Risk & Very Low Risk & Negligible Risk \\
\hline
\end{tabular}

\subsection{Validation Methodology}

Internal and external validation of models of social processes is crucial in establishing the reliability and explanatory power of these models. Models based on additive indices may be viewed as internally sensitive to the parameters chosen for each step of index construction. Therefore, attention should be paid to how each step of the index construction process (indicator selection, analysis scale selection, adjustment for data error/undercounts, normalization, variable transformation and weighting) influences the outcome of analysis. It has been shown that the sensitivity of additive index parameterization differs with each type of index type. For example, Tate (2012) applies variance-based global sensitivity analysis (Monte Carlo simulation ${ }^{20}$ ) to the most common additive index types using vulnerability/resilience data for different geographic locations, in order to uncover the nature of sensitivity inherent in each index type. Results showed that the hierarchical index model is highly affected by changes to

\footnotetext{
${ }^{20}$ A Monte Carlo simulation measures a model's sensitivity to small changes in one or more of its parameters, by running many thousands of model simulations with incremental modifications and collecting resulting statistics.
} 
variable weighting parameters, slightly affected by changes to variable transformation parameters, and negligibly affected by other construction steps (see Figure 9 below). As a consequence, Tate recommends additional methodological scrutiny at the variable weighting stage of hierarchical index design. Though not included in Tate's analysis, the step of choosing weights for sub-indices within a hierarchical index is assumed to also highly affect the outcome of analysis.

Figure 9. Sensitivity Analysis Comparison of 3 Common Additive Indices (Tate 2012)
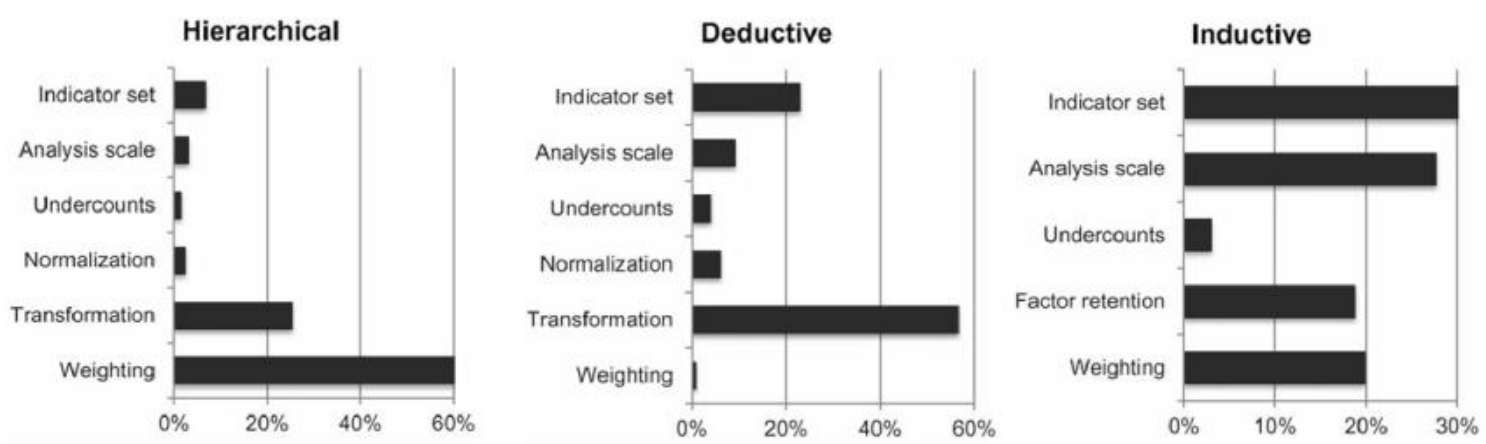

In the context of the proposed index construction methodology, sub-index weighting has received the least methodological attention, and therefore must be assumed to contribute the most uncertainty to the model. However, Monte Carlo-type sensitivity analysis of small weighting changes may be unnecessary here, since emergency managers are unlikely to make small changes to the weighting scheme of the proposed sub-indices. ${ }^{21}$ Also, absolute changes to a community's vulnerability/resilience

\footnotetext{
${ }^{21}$ For example, when choosing weights for the 'environmental' and 'socio-economic' sub-indices within the vulnerability index, it is assumed emergency managers will opt for multiples of 5 or 10 - such as applying a $40 \%$ weight to 'environmental' and $60 \%$ to 'socio-economic'. However, it is also assumed that the chosen weights may vary dramatically in different study areas, according to local conditions and the viewpoints of officials. For example, in a disaster-prone yet socially stable and prosperous area, the 'environmental' sub-index may be weighted $80 \%$ whereas the 'socio-economic' only $20 \%$.
} 
scores in the proposed dual-index approach are less important than changes to the resulting 'relative resilience' information categories each community falls into. Therefore, sensitivity analysis here will focus on how reasonable changes to sub-index weighting schemes in the vulnerability and resilience indices result in different categorization of communities in the final relative resilience matrix (see the proposed Confusion Matrix in Table 5 above). Since the objective of the proposed relative resilience categorization is to highlight communities with the most critical levels of risk (high vulnerability combined with low absolute resilience) in order to help prioritize mitigation efforts, the changes in total community population in high-risk categories after various sub-index weighting schemes will be presented.

\subsection{Evaluating Indicator Interaction, Cause-Effect Relationships and Key Indicators For Targeted Mitigation Strategies}

Once a model of community resilience has been built and validated, and after resilience scores for communities across a study area have been calculated, converted to information categories and mapped, disaster mitigation officials may still be quite uncertain about which indicators to target in order to increase the resilience of certain high-risk communities (those with high vulnerability and low resilience). After all, the final classification of a particular community's resilience is based on the interaction of quite a large set of variables ${ }^{22}$, each representing a real-world social process which exerts a certain amount of causal influence on other variables as well as the overall level

\footnotetext{
${ }^{22}$ Index-based resilience research in particular tends to include a high number of variables. Even in studies using the inductive approach to index construction, whereby a set of candidate study variables is whittled down through PCA analysis, the number of variables used can be in the dozens (c.f. Cutter 2007)
} 
of community resilience. For a mitigation effort to be maximally effective, it should be targeted at variables which not only are considered the most important to overall resilience, but also exert strong positive influence on other resilience indicators. Since the task of trying to think about how each variable influences all others would quickly become overwhelming, some tool for the structured analysis of these influences is required.

The DEMATEL method, extended for defining influence among resilience indicators by Hiete et al. (2011), is argued to be a promising tool for distinguishing the importance of resilience indicators, with the objective of communicating most influential resilience variables to decision makers. DEMATEL can be viewed as complimentary to AHP in an indicator analysis because it 1) shows how strongly each variable is related to all others, and 2) defines the nature of variable inter-influence by distinguishing between cause-and-effect indicators. This characterization of the indicators provides useful information for disaster management. If a mitigation effort is targeted at influencing an indicator which is both highly related to all others and of a causal nature, the overall policy impact is expected to be higher (Hiete et al. 2011). Similarly to AHP, the starting point of the DEMATEL method is a digraph, the direct relation matrix $\mathrm{M}$, which is a square matrix of $n \times n$ where $n$ is the total number of indicators in the index to be analyzed. ${ }^{23}$ To populate $M$, an analyst estimates the degree of direct influence between the different resilience indicators based on pair-wise

\footnotetext{
${ }^{23}$ As opposed to AHP, in which indicators are only compared with others within their respective sub-levels in separate digraphs, a DEMATEL analysis compares all variables in an index simultaneously in one digraph
} 
comparisons. The scale for quantification of influence may consist of integers which represent influence levels from low to high (such as $0=$ none or negligible influence, $1=$ low influence, 2 = medium influence, 3 = high influence, 4 = very high or controlling influence). Within $\mathrm{M}$, the $i^{\text {th }}$ row represents the quantified influence of indicator $i$ on each indicator $j=1, \ldots, n$. Since indicators are assumed not to influence themselves, all principal diagonal elements of $\mathrm{M}$ are set equal to zero.

Based on the methodology described by Fontela and Gabus (1976), M can be normalized as in Eq. 2.

$$
\left.N=\frac{M}{\max \left[\operatorname{maxi}=1, \cdots, n \sum_{j=1}^{n} \quad m \mathrm{i}, \mathrm{j}, \max =1, \ldots, \sum_{i=1}^{n} \quad m \mathrm{i}, \mathrm{j}\right.}\right]
$$

The normalized direct relation matrix $\mathrm{N}$ is then used to calculate the total directindirect relation matrix $\mathrm{T}$, depicting the direct and indirect influence among the indicators. The normalization of $\mathrm{N}$ ensures convergence of $\mathrm{T}$. For normalization, besides the maximum value of the row- and column-wise sums, the maximum value of just the row-wise sums can be used (Hu et al. 2009).

$$
T=\lim _{n \rightarrow \infty}\left(N+N^{2}+\cdots+N^{w}\right)=N(\mathrm{ld}-N)^{-1}
$$

Row- and column-wise summation of the elements of T gives the degree of dispatching $\mathrm{P}_{\mathrm{i}}$ or the degree of receiving $\mathrm{R}_{\mathrm{j}}$ which represent the total direct and indirect influence an indicator exerts on or receives from the other indicators (Tamura and Akazawa 2005).

$$
\begin{aligned}
& R_{i}=\sum_{i=1}^{n} t_{i, j} \\
& R_{j}=\sum_{j=1}^{n} t_{i, j}
\end{aligned}
$$


Furthermore, for each indicator the position value $s_{i}^{+}$and the relation value $s_{i}^{-}$ can be derived from the total direct-indirect relation matrix $\mathrm{T}$ as follows:

$$
\begin{gathered}
s_{i}^{+}=P_{i}+R_{i} \\
s_{i}^{+}=P_{i}+R_{i}
\end{gathered}
$$

The position value $s+i$ measures how strongly an indicator $i$ is related to all others. Indicators with low position values $s+i$ are neither strongly influenced by others nor do they influence other indicators to a considerable extent. The opposite is true for those with high $s+i$ values. The relationships captured by the positional values are therefore similar to those of internal statistical methods such as Pearson's correlation coefficient. However, its data-independence and exploitation of local knowledge allow the positional value to communicate perceived and directed influences, which may prove useful in a management context.

The relation values $s-i$ allow for distinguishing between cause and-effect group indicators. Indicators of the cause group influence others more than they are influenced themselves (therefore having positive net influence: $s_{i}>0$ ), whereas the effect group of indicators are defined as $s_{i}^{-}<0$ (negative net influence).

By plotting $\mathrm{s}^{+}$and $\mathrm{s}^{-}$i against each other, comparative assessment of the overall structural cause-effect relationships among indicators can be visualized in a causal diagram (Figure 10). As noted, mitigation policies directed at indicators exhibiting both high positional and relational values are expected to be more effective. Another way of visualizing the results of a DEMATEL analysis is by constructing an impact-relation network diagram from the total direct-indirect relation matrix T. All impact-relations 
can be shown simultaneously linked with directional arrows; or, using an arbitrary threshold value $s+i / s-j$, one can simplify the network diagram to show only the most 'important' impact relations (Li and Tzeng 2009, see Figure 11).

Figure 10. Example Casual Diagram Output from a DEMATEL Analysis.

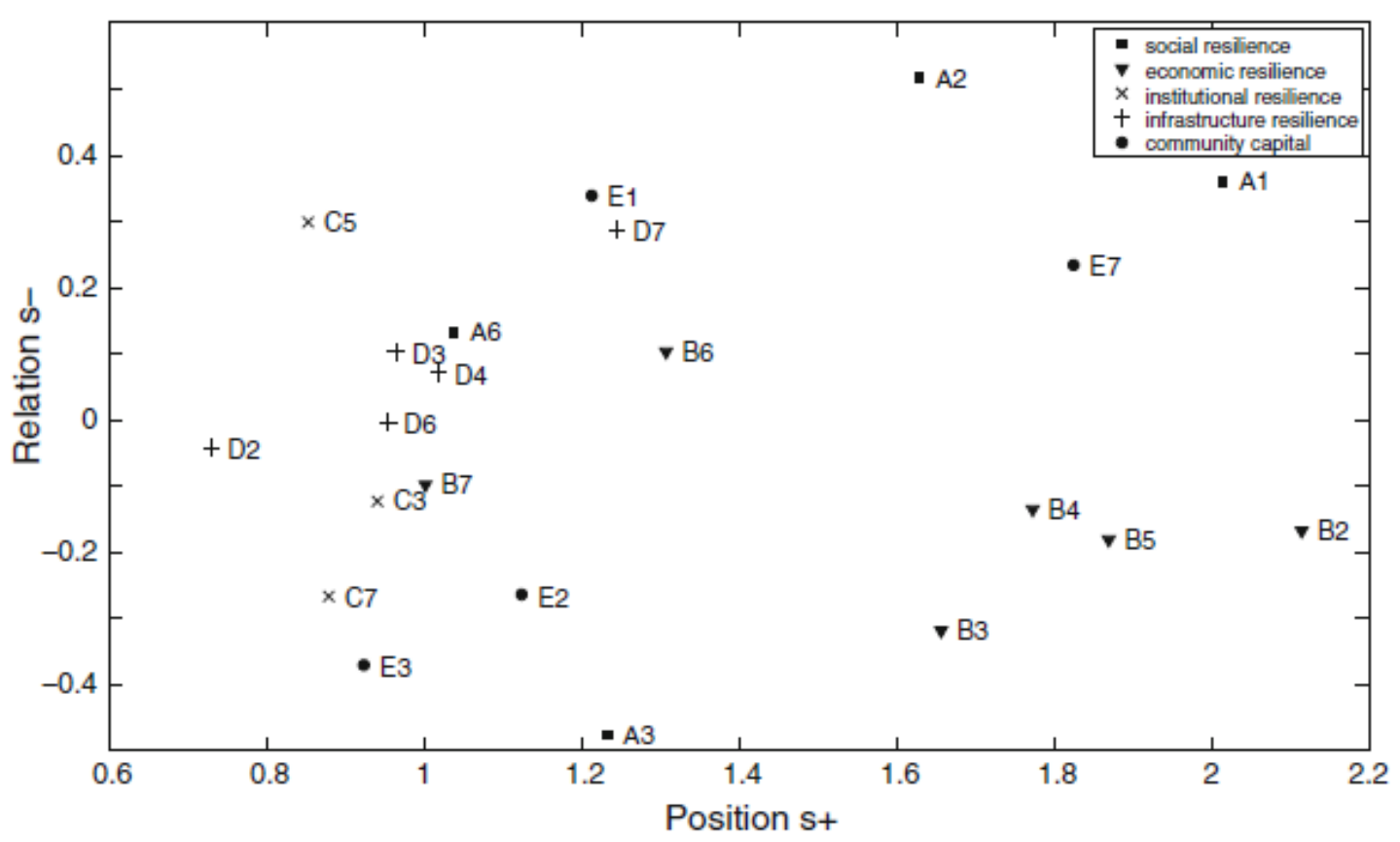

NOTE: Relational and positional values obtained from Eq. (7) and (8) are plotted against each other. In this example, variables A2, E7 and A1 have higher positional and relational values, signifying they are each related strongly to other variables and they each exert causal influence on a large number of other variables. Focusing on A2, E7 and/or A1 in mitigation efforts is expected to have a broader impact on the goal of increasing community resilience (Hiete et al. 2012). 
Figure 11. Directed-impact relations among variables from the T matrix can also be visualized in a network diagram. Below is an example diagram simplified with a threshold value of $s_{i}^{-} / s^{+}$in order to show only the most important relations (Hiete 2012)

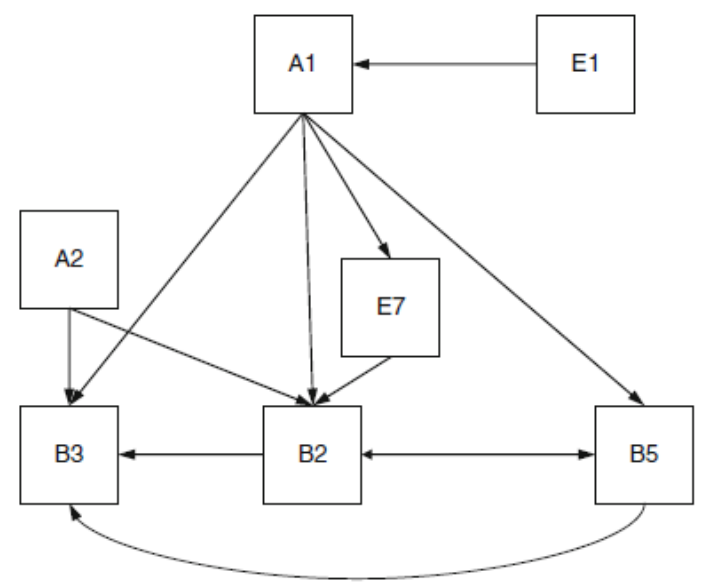




\section{CHAPTER 4. APPLICATION TO ST. LOUIS, MISSOURI}

\subsection{Study Area and Units of Analysis}

"St. Louis is a city of neighborhoods." - St. Louis City Department of Health (2012), Understanding Our Needs

Many data-intensive community resilience studies, as well as other public policyrelated research, use U.S. Census geographies such as tracts or postal zip codes as proxies for communities or urban neighborhoods (Krieger 2006). The advantages to that approach are, first, that Census tracts are designed to have similar populations which facilitates ease of comparing geographic differences, and second, that analysis at the Census tract level can be easily scaled up to larger Census geographies (Flanagan et al. 2011). However, an assumption in a community resilience study that Census tracts constitute distinct, self-identified communities, or that populations in those tracts can be reasonably expected to come together to solve problems, may be untenable. Therefore, this research will spatially define communities in St. Louis as St. Louisans do as neighborhoods. The rich history of St. Louis as an important gateway transit hub, as a show city for the combined 1904 Olympic Games and World's Fair, and as the site of many interesting architectural and urban design experiments may be too broad to cover here. However, one result of the city's cultural heritage is that many of its residents identify strongly with distinct neighborhoods. The city of St. Louis municipal government recognizes 79 neighborhoods, each with its own formative history and 
unique character. As such, official St. Louis neighborhood geographies will be utilized here (STL Planning Dept. 2010).

Figure 12. Study Area and Analysis Units- St. Louis City Neighborhoods (STL Planning Dept. 2010)

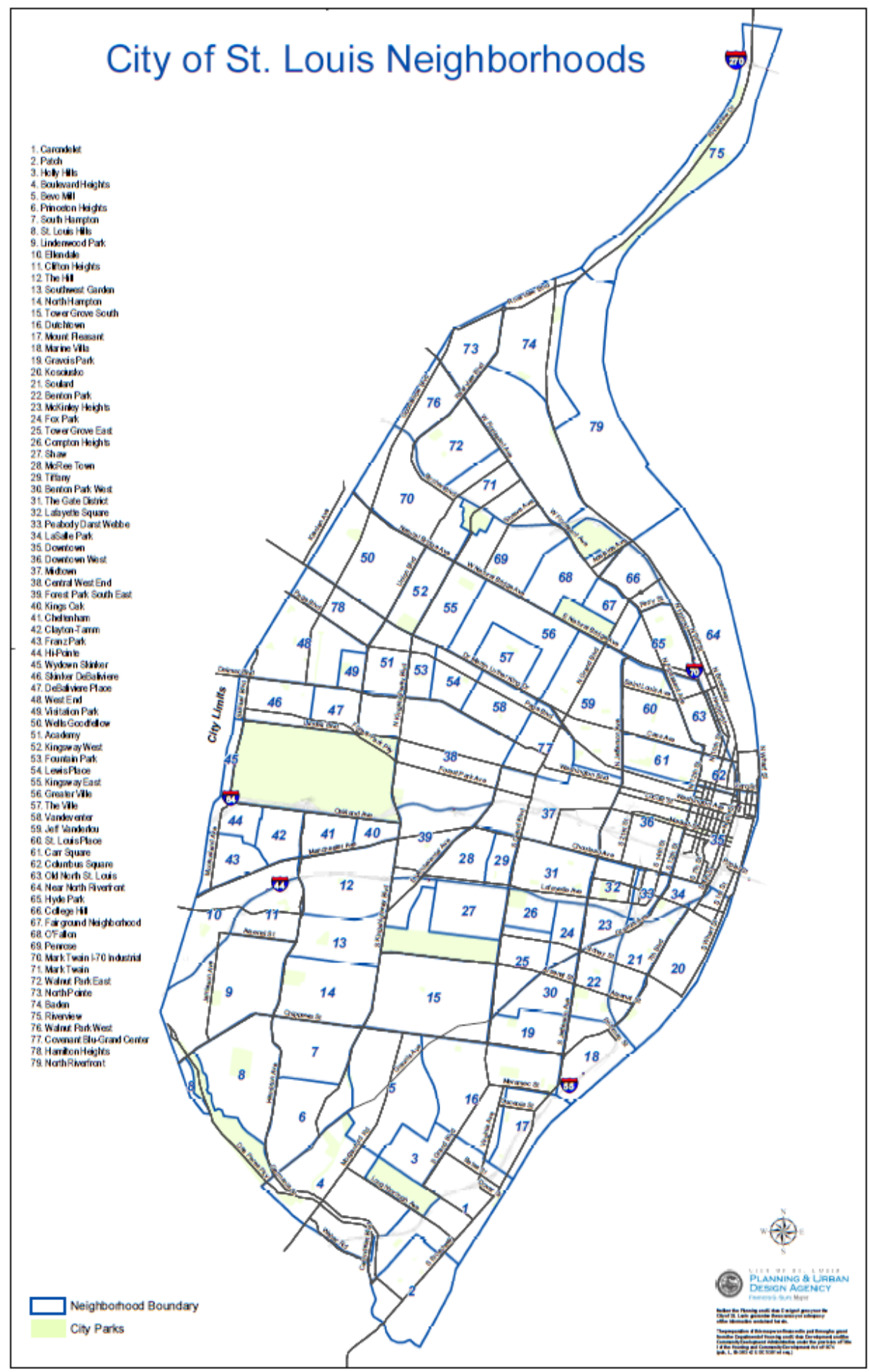




\subsection{Data Sources}

Selection of environmental hazards variables, and expert knowledge-based index weights for these variables in the vulnerability index, will be derived from the most recent (2009) St. Louis City Hazard Mitigation Plan (EW Gateway 2010b). East-West Gateway Council of Governments (EWG), a formal planning and coordination body made up of representatives from St. Louis-area municipal governments and school districts, takes on the responsibility of producing and submitting the Hazard Mitigation Plan to SEMA and FEMA on behalf of its constituent jurisdictions. Within this role, E-W Gateway also acts as the official public clearinghouse for all hazard, community profile, asset and mitigation planning data assembled for the FEMA report (EW Gateway 2010a). Before undertaking hazards data collection and research, EWG elicits input from members of constituent government agencies, local universities, and the general public (through a series of workshops and orientation meetings meant to gather mitigation issues, goals, priorities and ideas) (EW Gateway 2010a). Therefore, the hazards data, research methodology, risk assessments and mitigation priorities retained in EWG's Hazard Mitigation Plan represent a fusion of both expert and lay local knowledge of environmental hazards in the St. Louis metropolitan area. The St. Louis City section of the 2009 plan, including local emergency management officials' quantitative assessment of environmental hazard risks, is therefore utilized here to form a hazard profile of St. Louis City neighborhoods and to set weights for hazard variables within the environmental sub-index of the overall vulnerability index (see Tables 6 and 11 below). 
Socioeconomic vulnerability indicators are selected after consideration of local expert reporting of the main social stressors affecting populations in the City of St. Louis. Comprehensive socioeconomic threat data is published every three years by epidemiologists at the City of St. Louis Department of Health in the multi-volume report Understanding Our Needs (STL Dept. Health 2012). Though written by public health professionals, approximately half of this publication deals with deficiencies and vulnerabilities brought out by adverse socioeconomic conditions. Understanding Our Needs discusses conditions such as racial polarization, outmigration, poverty, teen pregnancy, crime, residential/business vacant space, hospital access, insurance coverage, public school quality, welfare recipients and many others. Therefore, indicators of social vulnerability from this publication are used to supplement other commonly used or recommended indicators in the disaster vulnerability literature (e.g. indicators of inequitable food access, English-language deficiency, low evacuation accessibility, infrastructure fragility, populations with functional disabilities, etc.). Data and variables used to represent chosen vulnerability indicators for this study are sourced primarily from the U.S. Census Bureau's American Community Survey 2011, as well as Missouri Department of Transportation, St. Louis Metropolitan Police Department, St. Louis City Departments of Planning, Urban Design and Health, National Association of Charter School Authorizers and The Reinvestment Fund. Since a multitude of variables exist that could denote resilience-boosting processes or qualities at each sub-level, they will be selected based on the author's expertise of the study area and publically-available data. Variables comprising the socio-economic sub-index of the 
overall vulnerability index are weighted using the AHP method described in Section 3.4, taking into account the perceived severity of these threats noted or implied in Understanding Our Needs, as well as the author's personal experience with social threats in the study area.

\subsection{Data Preparation}

Two neighborhoods within the City of St. Louis have no residential population (defined as civilian, non-institutionalized population) according to the ACS 2011. Kosciusko, constituting industrial and commercial land use, and has no reported residential population at all; North Riverfront contains a large institutionalized population (in the St. Louis City Correctional Facility) but no other reported residential population. It is certainly important to consider the disaster vulnerability/resilience of business entities and institutions within places such as Kosciusko and North Riverfront; however, since this thesis focuses primarily on qualities of people and processes in residential communities, these neighborhoods are omitted from analysis.

Since no Census-published data are available at the spatial scale of official St. Louis City neighborhoods, significant data preparation and restructuring is necessary to forge a correspondence between ACS 2011 geographies and the neighborhood analysis units. In consultation with demographers in the Research Division of St. Louis City Department of Planning, a system for redistributing data obtained in different Census areal units (such as block groups, tracts or postal zip codes) to neighborhood geographies was developed, using a relational database in a zonal GIS environment. 
This process (known as dasymmetric mapping - see Matisziw et al. 2008) can redistribute a certain proportion of tabular Census data (originally aggregated to block group, tract or zip code geographies) to neighborhood geographies based on the level of geometric intersection of these geographies. To account for the effects of the modifiable areal unit problem (MAUP), land use information on the locations of residential parcels is used to denote where within a Census geography the populations likely reside. In this application of dasymmetric mapping, there results a small residual of data that could not be allocated to the parcels since Census-developed Tiger/Line and St. Louis City-developed neighborhood spatial datasets not aligning exactly.

Once indicator variable data is prepared for both the vulnerability and resilience index, variables are reclassified to a value in the range $[0,1]$ with 0.0 representing the lowest and 1.0 as the highest vulnerability or resilience, respectively. In general, human variables representing counts of people or physical/spatial variables represented by land area were normalized by neighborhood population or land use totals. Variables representing road distances from neighborhoods to critical services or evacuation points are normalized by maximum observed distance values. Binary variables representing the presence or absence of a certain quality (such as an active neighborhood association) are coded as $[0,1]$ accordingly.

\subsection{Vulnerability Indicators and Index}


Table 6. Framework of Vulnerability: 'A Set of Threats'

\begin{tabular}{|c|c|c|c|c|c|}
\hline DIMENSION & ID & HAZARD INDICATOR & PROXY VARIABLE & JUSTIFICATION & DATA SOURCE \\
\hline \multirow{6}{*}{ ENVIRONMENTAL } & - & EARTHQUAKE & $\begin{array}{l}\text { LIQUEFACTION POTENTIAL AREASFROM } 7.8 \\
\text { MMI NEW MADRID EARTHQUAKE EVENT }\end{array}$ & - & $\begin{array}{l}\text { USGS 2012, EW GATEWAY } \\
2013\end{array}$ \\
\hline & - & EXTREME HEAT & $\begin{array}{l}\text { URBAN HEAT ISLAND POTENTIAL - } \\
\text { IMPERVIOUS LULC }\end{array}$ & - & $\begin{array}{c}\text { EPA-APPD, EW GATEWAY } \\
2013\end{array}$ \\
\hline & - & FLOOD & $\begin{array}{l}\text { RIVERINE, FLASH, AND L\&D\#327 FAILURE } \\
\text { PREDICTED FLOOD AREA }\end{array}$ & - & $\begin{array}{l}\text { FEMA 2013, EW GATEWAY } \\
2013 \\
\end{array}$ \\
\hline & - & $\begin{array}{l}\text { INDUSTR. POLLUTANT } \\
\text { EXPOSURE }\end{array}$ & $\begin{array}{l}\text { ENVIRONMENTAL HEALTH HAZARD INDEX } \\
\text { CATEGORY AREAS }\end{array}$ & - & EW GATEWAY 2013 \\
\hline & - & $\begin{array}{l}\text { TORNADO + WIND/HAIL } \\
\text { STORM }\end{array}$ & $\begin{array}{l}\text { INVARIABLE - WEIGHTED BY EXPERT RISK } \\
\text { ASSESSMENT }\end{array}$ & - & EW GATEWAY 2013 \\
\hline & - & EXTREME COLD & $\begin{array}{l}\text { INVARIABLE - WEIGHTED BY EXPERT RISK } \\
\text { ASSESSMENT }\end{array}$ & - & EW GATEWAY 2013 \\
\hline \multirow{13}{*}{$\begin{array}{l}\text { SOCIO- } \\
\text { ECONOMIC }\end{array}$} & V1 & AGING STRUCTURES & \% HOMES BUILT BEFORE 1940 & MILETI 1999 & ACS 2011 \\
\hline & V2 & PUBLIC SAFETY THREAT & $\begin{array}{l}\text { AVERAGE ANNUAL VIOLENT \& MAJOR } \\
\text { PROPERTY CRIMES 2007-2011 }\end{array}$ & SHAW-TAYLOR 1999 & SLMPD 2013 \\
\hline & V3 & $\begin{array}{l}\text { ECONOMIC INSTABILITY - } \\
\text { INSUFFICIENT INCOME }\end{array}$ & $\begin{array}{c}\text { POVERTY RATE (\% POP BELOW POVERTY } \\
\text { LEVEL) }\end{array}$ & HARKNESS 2007 & ACS 2011 \\
\hline & V4 & UNEMPLOYMENT RATE & $\%$ UNEMPLOYED & TIERNEY 2007 & ACS 2011 \\
\hline & V5 & $\begin{array}{l}\text { VULNERABLE HOUSEHOLDS - } \\
\text { SINGLE MOTHER HH } \\
\end{array}$ & $\begin{array}{l}\text { \% FAMILIES WITH SINGLE FEMALE HEAD OF } \\
\text { HOUSEHOLD WITH DEPENDENT CHILDREN }\end{array}$ & MORROW 1999 & ACS 2011 \\
\hline & V6 & $\begin{array}{l}\text { VULNERABLE HOUSEHOLDS - } \\
\text { SPECIAL NEEDS }\end{array}$ & $\begin{array}{l}\text { \% ELDERLY WHO ARE DISABLED AND LIVING } \\
\text { ALONE }\end{array}$ & HEINZ CENTER 2002 & ACS 2011 \\
\hline & V7 & $\begin{array}{l}\text { ECONOMIC INSTABILITY - } \\
\text { COMMERCIAL VACANCY }\end{array}$ & $\begin{array}{l}\text { \% LONG-TERM VACANT COMMERCIALLY- } \\
\text { ZONED PROPERTIES (STRATEGIC LAND USE) }\end{array}$ & ST. LOUIS CITY 2012 & $\begin{array}{l}\text { ST. LOUIS DEPT OF PLANNING } \\
2012 \\
\end{array}$ \\
\hline & V8 & $\begin{array}{l}\text { ECONOMIC INSTABILITY - } \\
\text { RESIDENTIAL VACANCY }\end{array}$ & $\begin{array}{l}\text { \% LONG-TERM VACANT RESIDENTIALLY- } \\
\text { ZONED PROPERTIES (STRATEGIC LAND USE) }\end{array}$ & ST. LOUIS CITY 2012 & $\begin{array}{l}\text { ST. LOUIS DEPT OF PLANNING } \\
2012 \\
\end{array}$ \\
\hline & V9 & INEQUITABLE FOOD ACCESS & $\begin{array}{l}\text { \% OF NEIGHBORHOOD WITHIN LIMITED } \\
\text { SUPERMARKET ACCESS AREAS (LSA) }\end{array}$ & TRF 2013 & TRF 2013 \\
\hline & V10 & POOR SCHOOL QUALITY & $\begin{array}{l}\text { SERVICE GAP IN MISSOURI TIER } 1 \mathrm{~K}-12 \\
\text { SCHOOLS }\end{array}$ & NACSA 2009 & NACSA 2009 \\
\hline & V11 & $\begin{array}{l}\text { LIMITED ENGLISH } \\
\text { PROFICIENCY }\end{array}$ & $\begin{array}{c}\% \text { ESL + NON-ENGLISH SPEAKING } \\
\text { POPULATION } \\
\end{array}$ & MORROW 2008 & ACS 2011 \\
\hline & V12 & EVAC. POTENTIAL - ACCESS & $\begin{array}{l}\text { PRINCIPAL ARTERIAL MILES PER } \\
\text { NEIGHBORHOOD }\end{array}$ & NRC 2006 & MODOT 2013 \\
\hline & V13 & $\begin{array}{l}\text { EMERGENCY MEDICAL } \\
\text { ACCESSIBILITY }\end{array}$ & $\begin{array}{c}\text { DRIVE TIME FROM NEIGHBORHOOD } \\
\text { CENTROID TO NEAREST ER-EQUIPPED } \\
\text { HOSPITAL }\end{array}$ & $\begin{array}{l}\text { AUF DER HYDE AND } \\
\text { SCANLON } 2007\end{array}$ & $\begin{array}{l}\text { GIS DERIVED FROM MODOT, } \\
\text { STATE OF MO PUBLIC HEALTH }\end{array}$ \\
\hline
\end{tabular}

Table 7. Digraph Structure for AHP Assessment of Socio-economic Vulnerability Sub-Index

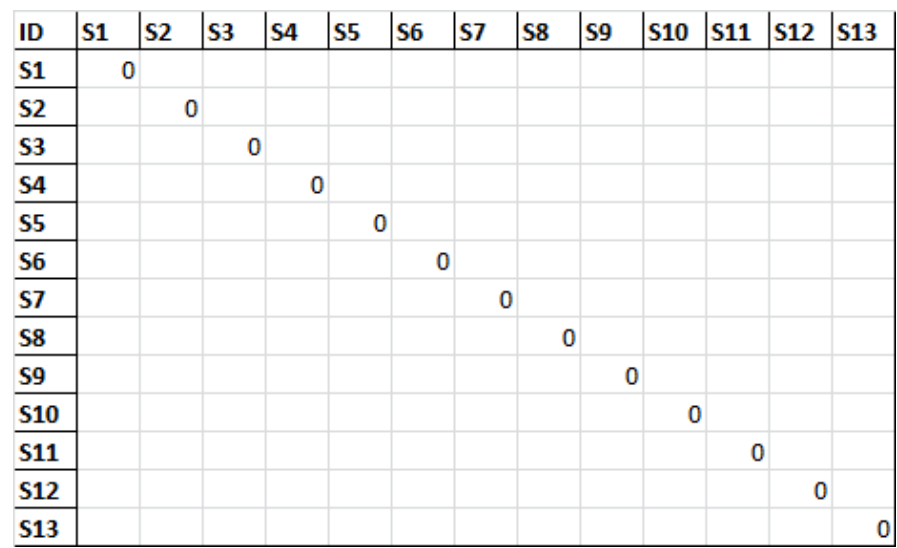

Note: AHP assessment is used to set indicator weights in the Socio-Economic sub-level of the vulnerability index (indicators in the Environmental sub-index are weighted separately using already-available quantified expert judgments from the St. Louis Hazard Mitigation Plan shown in Table 11). Each cell is filled in with a quantified judgment of the level of threat of indicator $i$ (row) as opposed to indicator $j$ (column), as described in Section 3.4, using the scale in Table 3. 


\subsection{Resilience Indicators and Index}

Table 8. Framework of Resilience: 'A Set of Protections'

\begin{tabular}{|c|c|c|c|c|c|}
\hline LEVEL & ID & INDICATOR & VARIABLE & JUSTIFICATION & DATA SOURCE \\
\hline \multirow{8}{*}{$\begin{array}{l}\text { INDIVIDUAL \& } \\
\text { HOUSEHOLD }\end{array}$} & I1 & CIVIC PARTICIPATION & \% CENSUS 2010 PARTICIPATION & CUTTER 2010 & US CENSUS BUREAU 2011 \\
\hline & 12 & EDUCATIONAL ATTAINMENT & $\begin{array}{c}\text { \% POPULATION HAVING AT LEAST SOME COLLEGE } \\
\text { EDUCATION }\end{array}$ & UNDP 1990 & ACS 2011 \\
\hline & 13 & LOW INCOME BUFFER & $\begin{array}{l}\text { AVE HH GOV ASSISTANCE DOLLARS PER HHS IN } \\
\text { POVERTY }\end{array}$ & $\begin{array}{c}\text { BIANCHI ET AL } \\
1982\end{array}$ & ACS 2011 \\
\hline & 14 & INSURANCE COVERAGE & $\begin{array}{c}\text { \% CIVILIAN POP COVERED BY ANY TYPE OF } \\
\text { HEALTH INSURANCE PLAN }\end{array}$ & DORFMAN 1979 & ACS 2011 \\
\hline & 15 & HH ECONOMIC SECURITY & $\begin{array}{l}\text { \% HOMEOWNERS NOT SEVERELY COST- } \\
\text { BURDENED }\end{array}$ & $\begin{array}{l}\text { BIANCHI ET AL } \\
1982\end{array}$ & ACS 2011 \\
\hline & 17 & $\begin{array}{c}\text { ELDERLY + DISABLED POPULATION } \\
\text { CONNECTIVITY }\end{array}$ & \# SLAAA FUNCTIONAL NEEDS REGISTRANTS & $\begin{array}{l}\text { MCGUIRE ET AL } \\
2007\end{array}$ & SLAAA 2013 \\
\hline & 18 & EMERGENCY MOBILITY & $\%$ HH WITH A CAR & TIERNEY 2007 & ACS 2011 \\
\hline & 19 & PLACE ATTACHMENT & $\%$ HH RESIDING > 10 YEARS & $\begin{array}{c}\text { VALE \& } \\
\text { CAMPANELLA } 2005\end{array}$ & ACS 2011 \\
\hline \multirow{11}{*}{$\begin{array}{c}\text { COMMUNITY } \\
\text { (NEIGHBORHOOD) }\end{array}$} & N1 & $\begin{array}{l}\text { COMMUNITY INVOLVEMENT - } \\
\text { RESIDENTIAL }\end{array}$ & ACTIVE NEIGHBORHOOD ASSOCIATION & $\begin{array}{l}\text { STEWART ET AL } \\
2009\end{array}$ & SLACO 2013 \\
\hline & N2 & $\begin{array}{l}\text { COMMUNITY INVOLVEMENT - PLACE } \\
\text { ATTACHMENT / FOOD SECURITY }\end{array}$ & ACTIVE COMMUNITY GARDEN & $\begin{array}{l}\text { TIDBAL AND } \\
\text { KRASNEY } 2007\end{array}$ & GATEWAY GREENING 2013 \\
\hline & N3 & $\begin{array}{l}\text { COMMUNITY INVOLVEMENT - } \\
\text { ECONOMIC PRESERVATION \& } \\
\text { DEVELOPMENT }\end{array}$ & ACTIVE NEIGHBORHOOD BUSINESS ASSOCIATION & $\begin{array}{l}\text { STEWART ET AL } \\
2009\end{array}$ & SLACO 2013 \\
\hline & N4 & $\begin{array}{l}\text { COMMUNITY IDENTITY \& PLACE } \\
\text { ATTACHMENT }\end{array}$ & $\begin{array}{l}\text { HISTORIC PLACE OR DISTRICT (NRHP REGISTRY) } \\
\text { INSIDE NEIGHBORHOOD }\end{array}$ & $\begin{array}{l}\text { FLANAGAN ET AL } \\
2011\end{array}$ & $\begin{array}{l}\text { NRHP 2013, EW GATEWAY } \\
\text { 2012, MSDIS, } 2013\end{array}$ \\
\hline & N5 & $\begin{array}{c}\text { CIVIC PARTICIPATION / POLITICAL } \\
\text { ENGAGEMENT }\end{array}$ & $\begin{array}{c}\text { ACTIVE WARD ASSOCIATION - POLITICAL } \\
\text { PARTISAN ORGANIZATIONS }\end{array}$ & $\begin{array}{l}\text { STEWART ET AL } \\
2009 \\
\end{array}$ & ST. LOUIS CITY HALL \\
\hline & N6 & NEIGHBORHOOD RACIAL DIVERSITY & GINI COEFFICIENT - 'WHITE','BLACK', 'OTHER' & NORRIS ET AL 2008 & ACS 2011 \\
\hline & N7 & NEIGHBORHOOD AGE EQUITY & $\begin{array}{c}\text { GINI COEFFICIENT - '0-14','15-34','35-54',55- } \\
74^{\prime}, 754^{\prime}\end{array}$ & MORROW 2008 & ACS 2011 \\
\hline & N8 & NEIGHBORHOOD INCOME DIVERSITY & $\begin{array}{l}\text { GINI COEFFICIENT - } 6 \text { CATEGORIES (BELOW } \\
\text { POVERTY, LOW, LOW-MEDIAN, HIGH-MEDIAN, } \\
\text { HIGH, WEALTHY }\end{array}$ & DORFMAN 1979 & ACS 2011 \\
\hline & N9 & $\begin{array}{l}\text { NEIGHBORHOOD OCCUPATIONAL } \\
\text { DIVERSITY }\end{array}$ & $\begin{array}{c}\text { GINI COEFFICIENT - 'BUSINESS SCIENCE \& } \\
\text { ARTS','SERVICE', 'SALES \& OFFICE', } \\
\text { 'MANUFACTURING \& EXTRACTION', } \\
\text { 'TRANSPORTATION } \\
\end{array}$ & $\begin{array}{l}\text { BOLLMAN ET AL } \\
2006\end{array}$ & ACS 2011 \\
\hline & N10 & $\begin{array}{c}\text { ECONOMIC RESILIENCE - BUSINESS SIZE } \\
\text { DIVERSITY }\end{array}$ & $\begin{array}{c}\text { GINI COEFFICIENT - BUSINESS SIZES '1-19','20- } \\
99^{\prime}, 100+{ }^{\prime}\end{array}$ & $\begin{array}{l}\text { GARMESTANI ET AL } \\
2006\end{array}$ & ESRI BUSINESS ANALYST 2013 \\
\hline & N11 & $\begin{array}{c}\text { ECONOMIC RESILIENCE - INNOVATIVE } \\
\text { POTENTIAL }\end{array}$ & $\begin{array}{l}\text { \% POP EMPLOYED IN STEM-RELATED } \\
\text { OCCUPATIONS }\end{array}$ & $\begin{array}{l}\text { MCGRANAHAN \& } \\
\text { WOJAN } 2007\end{array}$ & BLS 2012, ACS 2011 \\
\hline \multirow{7}{*}{$\begin{array}{l}\text { GOVERNMENT \& } \\
\text { INSTITUTIONAL }\end{array}$} & G3 & $\begin{array}{c}\text { GOVERNMENT EXTREME } \\
\text { TEMPERATURE MITIGATION EFFORT }\end{array}$ & WARMING AND COOLING SHELTER & EW GATEWAY 2009 & $\begin{array}{l}\text { STATE OF MO, UNITED WAY } \\
2013\end{array}$ \\
\hline & G4 & $\begin{array}{c}\text { GOVERNMENT EMERGENCY FOOD } \\
\text { MITIGATION EFFORT }\end{array}$ & EMERGENCY SUPPLEMENTAL FOOD SITE & TRF 2013 & $\begin{array}{l}\text { STATE OF MO, UNITED WAY } \\
2013\end{array}$ \\
\hline & G5 & $\begin{array}{l}\text { GOVERNMENT GENDER-BASED } \\
\text { EMERGENCY MITIGATION EFFORT }\end{array}$ & WOMEN AND CHILDREN CRISES CENTER & MORROW 1999 & $\begin{array}{l}\text { STATE OF MO, UNITED WAY } \\
2013\end{array}$ \\
\hline & G6 & $\begin{array}{c}\text { GOVERNMENT- } \\
\text { PROVIDEDMULTIPURPOSE SPACE }\end{array}$ & GOVERNMENT-MAINTAINED OPEN SPACE & $\begin{array}{l}\text { STEWART ET AL } \\
2009\end{array}$ & $\begin{array}{c}\text { CITY OF ST LOUIS PLANNING } \\
\text { DEPT 2013, EW GATEWAY } \\
2013 \\
\end{array}$ \\
\hline & G7 & INST. EMERGENCY HEALTH MITIGATION & COMMUNITY HEALTH CENTER & CUTTER 2010 & $\begin{array}{c}\text { ST LOUIS CITY DEPT OF } \\
\text { HEALTH } 2013\end{array}$ \\
\hline & G8 & $\begin{array}{l}\text { INST. NON-PROFIT/CIVIC } \\
\text { REPRESENTATION }\end{array}$ & \# OF CIVIC ORGS PER 10,000 POPULATION & MURPHY 2007 & NAICS 2012 \\
\hline & G9 & INST. CAPITAL DEVELOPMENT & $\begin{array}{l}\text { \# OF CONSUMER SAVINGS BANKS WITHIN } \\
\text { NEIGHBORHOOD }\end{array}$ & $\begin{array}{l}\text { LONGSTAFF ET AL } \\
2010\end{array}$ & FDIC 2013 \\
\hline
\end{tabular}


Table 9. Digraph Structures for AHP Assessment of Resilience Sub-Indices

A. \begin{tabular}{r|r|l|l|l|l|l|l|l|l|}
\hline $\begin{array}{c}\text { Individual / } \\
\text { Household }\end{array}$ & 11 & 12 & 13 & 14 & 15 & 16 & 17 & 18 & 19 \\
\hline
\end{tabular}

\begin{tabular}{|c|l|l|l|l|l|l|l|l|l|}
\hline 11 & 0 & & & & & & & & \\
\hline 12 & & 0 & & & & & & & \\
\hline 13 & & & 0 & & & & & & \\
\hline 14 & & & & 0 & & & & & \\
\hline 15 & & & & & 0 & & & & \\
\hline 16 & & & & & & 0 & & & \\
\hline 17 & & & & & & & 0 & & \\
\hline 18 & & & & & & & & 0 & \\
\hline 19 & & & & & & & & & 0 \\
\hline
\end{tabular}

B. \begin{tabular}{c|c|c|c|c|c|c|c|c|c|c|c|}
\hline $\begin{array}{c}\text { Neighborhood/ } \\
\text { Community }\end{array}$ & N1 & N2 & N3 & N4 & N5 & N6 & N7 & N8 & N9 & N10 & N11 \\
\hline
\end{tabular}

\begin{tabular}{|c|l|l|l|l|l|l|l|l|l|l|l|}
\hline $\begin{array}{c}\text { Community } \\
\text { N1 }\end{array}$ & N1 & N2 & N3 & N4 & N5 & N6 & N7 & N8 & N9 & N10 & N11 \\
\hline N2 & & 0 & & & & & & & & & \\
\hline N3 & & & 0 & & & & & & & & \\
\hline N4 & & & & 0 & & & & & & & \\
\hline N5 & & & & & 0 & & & & & & \\
\hline N6 & & & & & & 0 & & & & & \\
\hline N7 & & & & & & & 0 & & & & \\
\hline N8 & & & & & & & & 0 & & & \\
\hline N9 & & & & & & & & & 0 & & \\
\hline N10 & & & & & & & & & 0 & \\
\hline N11 & & & & & & & & & & & 0 \\
\hline
\end{tabular}

C.

\begin{tabular}{|c|c|c|c|c|c|c|c|c|c|}
\hline $\begin{array}{c}\text { Government / } \\
\text { Institutional }\end{array}$ & G1 & G2 & G3 & G4 & G5 & G6 & G7 & G8 & G9 \\
\hline G1 & 0 & & & & & & & & \\
\hline G2 & & 0 & & & & & & & \\
\hline G3 & & & 0 & & & & & & \\
\hline G4 & & & & 0 & & & & & \\
\hline G5 & & & & & 0 & & & & \\
\hline G6 & & & & & & 0 & & & \\
\hline G7 & & & & & & & 0 & & \\
\hline G8 & & & & & & & & 0 & \\
\hline G9 & & & & & & & & & 0 \\
\hline
\end{tabular}

Note: AHP assessment is used to set indicator weights in each of three sub-levels of the resilience index (referenced above as A., B. and C.). Each cell is filled in with a quantified judgment of the importance of $i$ (row) vs $j$ (column), as described in Section 3.4, using the scale in Table 3. 
Table 10. Digraph Structure for DEMATEL Analysis of Resilience Indicators

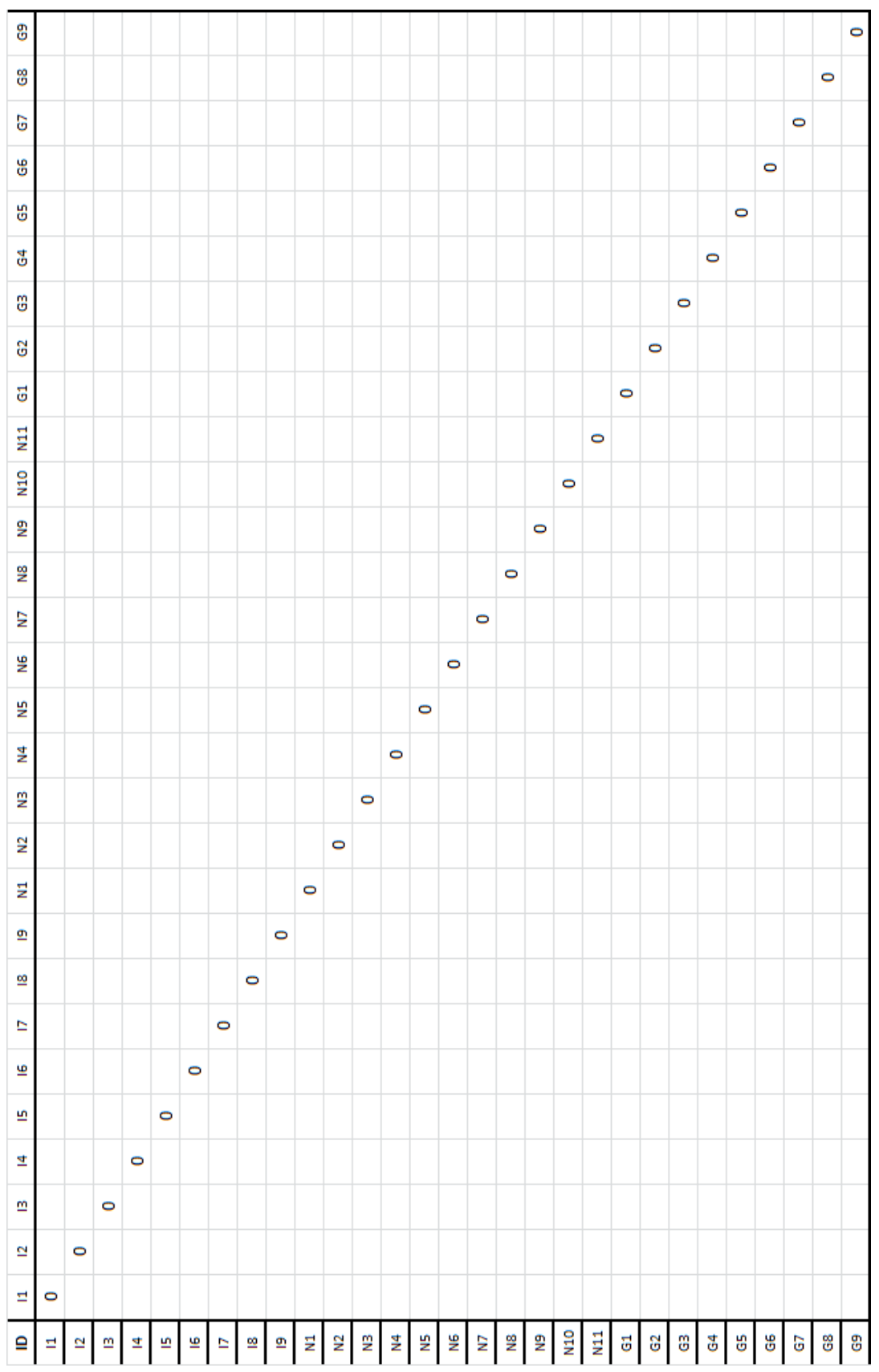

NOTE: DEMATEL analysis is used to both quantify relationships/influences among resilience indicators and to reveal which indicators are of a more causal nature and therefore more important in a management context. Each cell is filled in with a quantified judgment of the amount of influence $i$ (row) exerts on $j$ (column), with $0.0=$ no or negligible influence, $1.0=$ low influence, $2.0=$ medium influence, $3.0=$ high influence, and 4.0 = very high or controlling influence. The results of DEMATEL are useful after each neighborhood's relative resilience has been calculated, because it can help answer the question: "Which indicators would have the highest impact on overall resilience if they were strengthened?" Mitigation strategies for neighborhoods with low relative resilience scores are better directed at these high-impact indicators. 


\subsection{Validation Datasets}

Although the concept of population disaster resilience has sometimes been described more in terms of its intangibility than its observability, external validation of a resilience model using empirical evidence of actual disaster resilience may yet be possible. Such validation can be conducted for St. Louis, using publically-available data on disaster-related deaths and requests for assistance. The dataset representing observations of disaster resilience is comprised of 18 geo-coded occurrences of heatrelated fatalities during the intense heat wave which affected the St. Louis area in summer 2012 (St. Louis Post-Dispatch 2012). Geographically-weighted regression was conducted using aggregated heat fatalities per neighborhood as the dependent variable, and relative resilience score as the explanatory variable. Likewise, relative resilience scores of neighborhoods may also be evaluated as an explanatory variable for recent population change (outmigration) of St. Louis neighborhoods. Supplementing this study area-wide validation, other datasets representing observed disaster resilience in more specific areas can be used, such as resident requests for assistance from St. Louis City Emergency Management Agency (CEMA) following windstorms in north St. Louis in 2011 (St. Louis CSB 2013).

Since this thesis argues that the proposed methods for modelling community resilience - which are based primarily on assessments of local knowledge as opposed to internal statistics - can explain observed resilience better than current simple additive index techniques, a comparison will be made between this study's output 
characterization of relative community resilience and an analysis using data and index methods similar to the dominant geographic approach. 


\section{CHAPTER 5. RESULTS}

5.1 Hazard Profile, Vulnerability Index and Sub-Index Weights

Table 11. Results of Environmental Hazard Assessment from City of St. Louis Mitigation Plan with Calculated Weights for Vulnerability Index (EW Gateway 2010a)

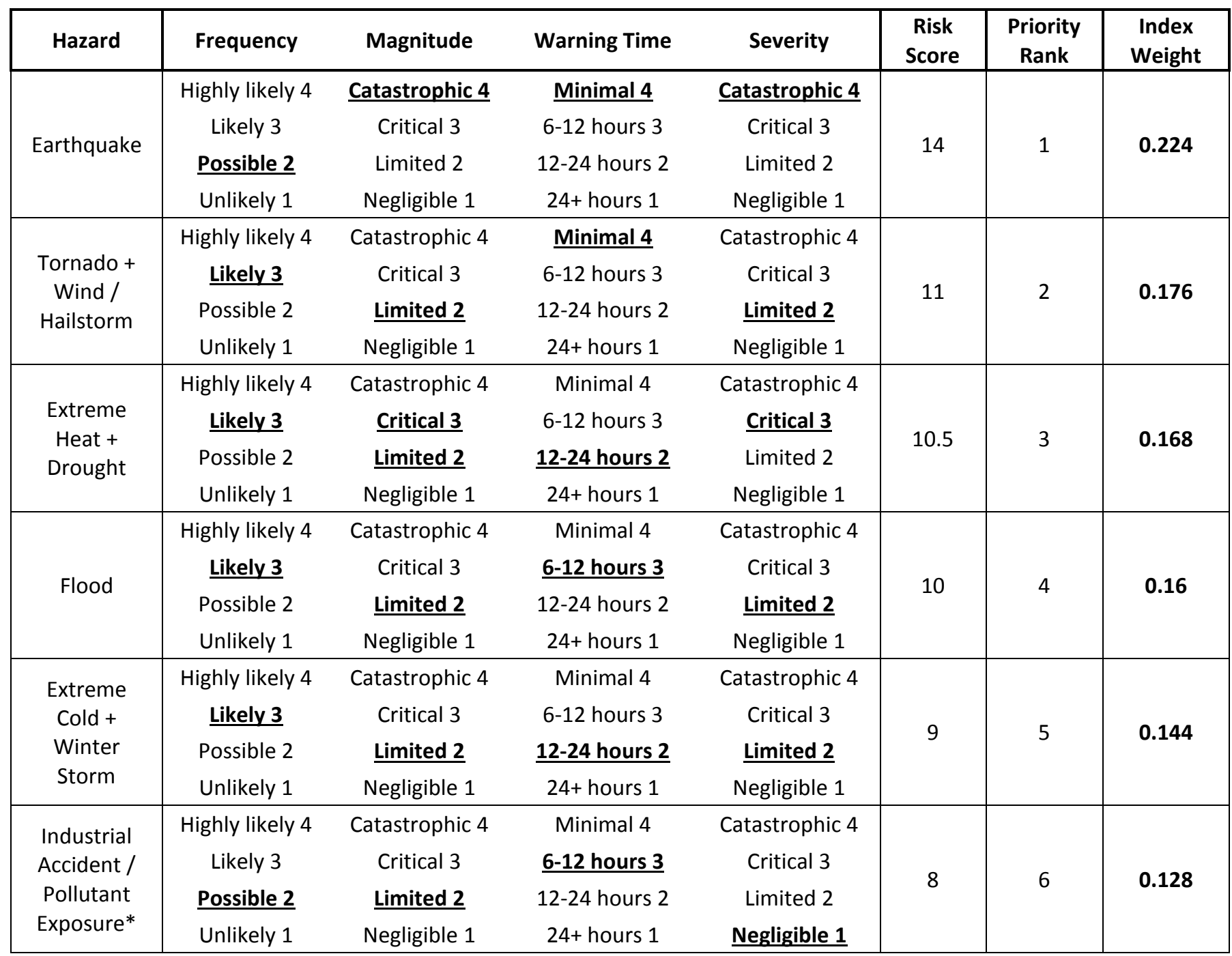

*Industrial Accident / Pollution Exposure hazard was added by the current author due to EWG's intended inclusion of this hazard in the 2015 Hazard Mitigation Plan. This hazard's characteristics were assessed by the author using EWG's 2012 Health Hazard Index dataset and accompanying report (see Appendix for a map of the index per block group overlaid with St. Louis neighborhoods).

NOTE: Each hazard indicator's Index Weight is calculated as its Risk Score divided by the Risk Score column total (62.5), which ensures all indicator weights sum to 1.0. 
Table 12. Results of AHP Assessment of Socio-Economic Vulnerability Indicators (See Table 6 in Chapter 4 for List of Socio-Economic Indicators and Variables)

\begin{tabular}{c|c|c|}
\hline ID & INDICATOR & WEIGHT \\
\hline S5 & $\begin{array}{c}\text { Vulnerable Households - Single } \\
\text { Mother With Children }\end{array}$ & 0.11216 \\
\hline S10 & Poor School Quality & 0.10621 \\
\hline S2 & Public Safety Threat & 0.10565 \\
\hline S3 & $\begin{array}{c}\text { Economic Instability - } \\
\text { Insufficient Income }\end{array}$ & 0.09761 \\
\hline S4 & Unemployment Riate & 0.08734 \\
\hline S8 & $\begin{array}{c}\text { Economic Instability - } \\
\text { Residential Vacancy }\end{array}$ & 0.08170 \\
\hline S6 & $\begin{array}{c}\text { Vulnerable Households - } \\
\text { Special Needs Elderly }\end{array}$ & 0.07511 \\
\hline S7 & $\begin{array}{c}\text { Economic Instability - } \\
\text { Commercial Vacancy }\end{array}$ & 0.06827 \\
\hline S9 & Inequitable Food Access & 0.06090 \\
\hline S13 & Emergency Medical & 0.05985 \\
\hline S12 & Evacuation Potential & 0.05110 \\
\hline S1 & Aging Structures & 0.05094 \\
\hline S11 & Limited English Proficiency & 0.04315 \\
\hline
\end{tabular}

Figure 13. Distribution and Standard Deviation Categories of Vulnerability Index

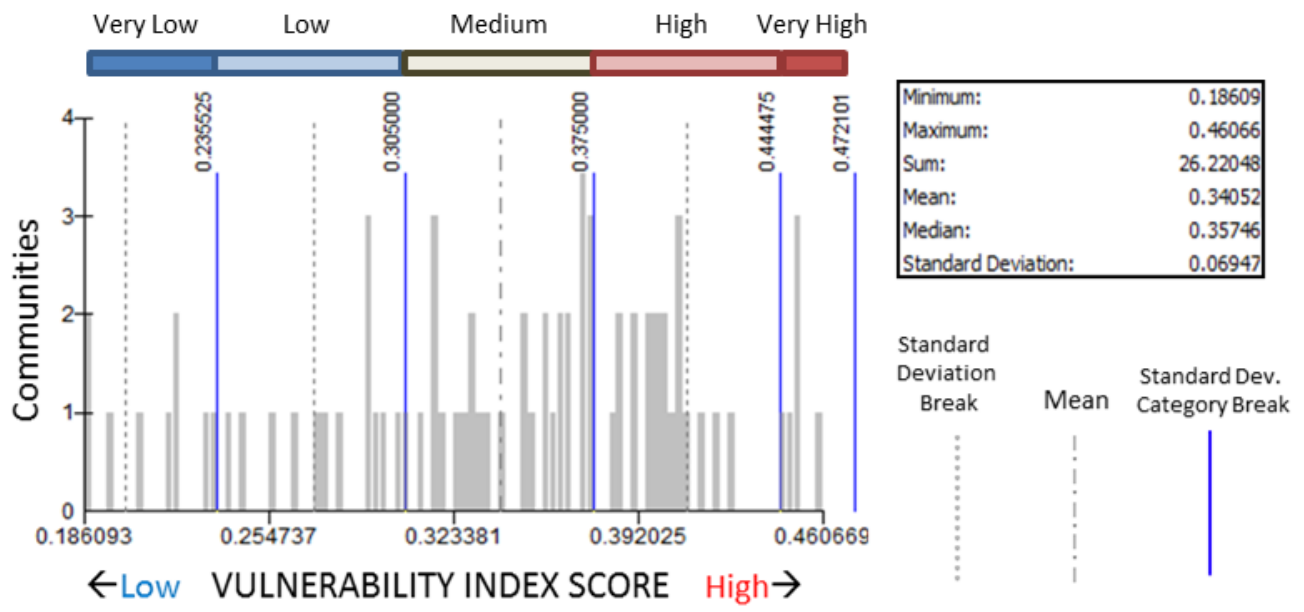


Figure 14. Map of Neighborhood Hazard Vulnerability

\section{Hazard Vulnerability of St. Louis Neighborhoods}
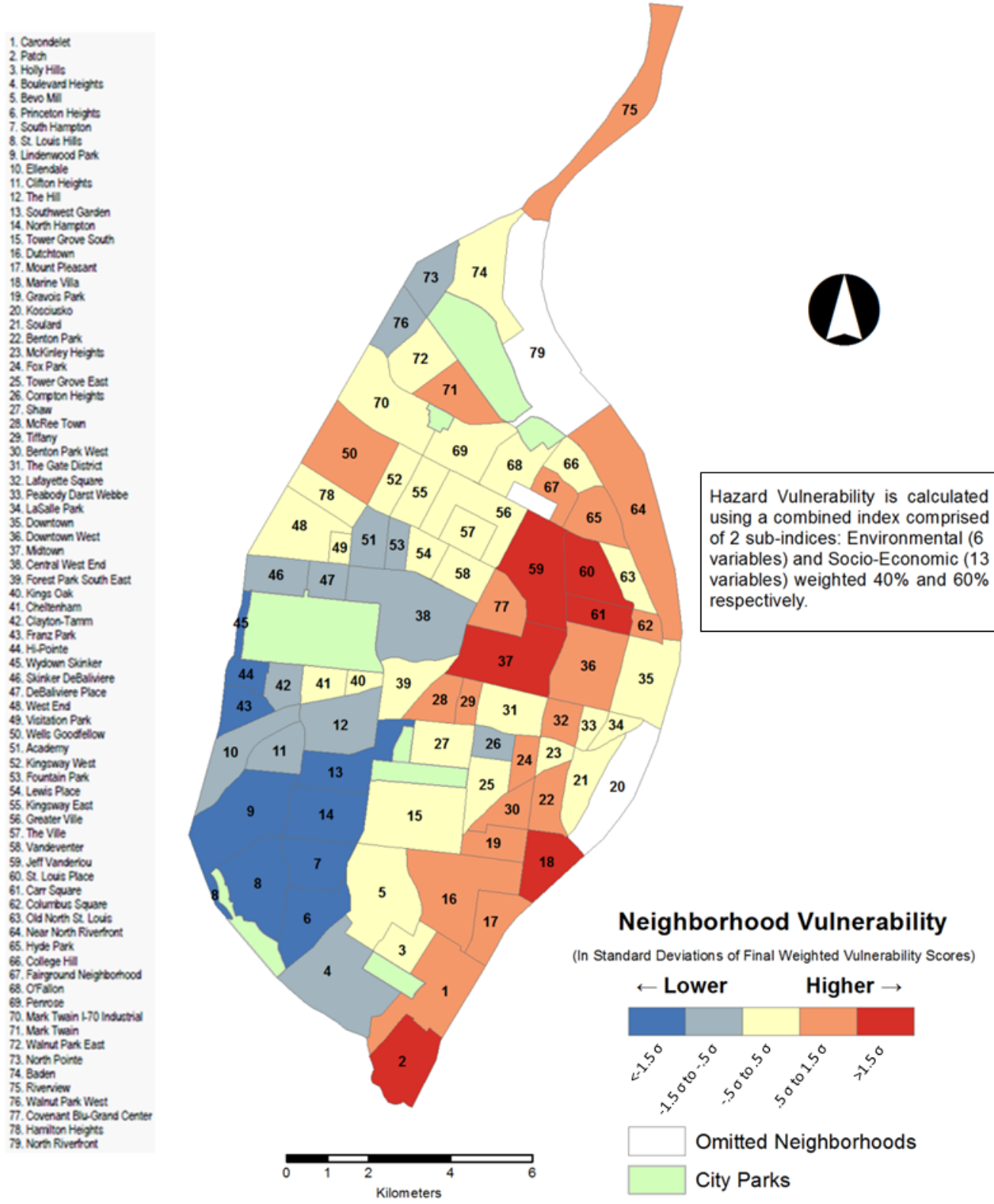


\subsection{Absolute Resilience Index and Sub-Index Weights}

Table 13. Results of AHP Assessment of Resilience Index Sub-Levels

A.

\begin{tabular}{|c|c|c|}
\hline \multicolumn{3}{|c|}{ INDIVIDUAL / HOUSEHOLD } \\
\hline ID & Indicator & Weight \\
\hline 12 & $\begin{array}{l}\text { Educational } \\
\text { Attainment }\end{array}$ & 0.16125 \\
\hline 14 & $\begin{array}{l}\text { Insurance } \\
\text { Coverage }\end{array}$ & 0.14637 \\
\hline 15 & $\begin{array}{l}\text { Household } \\
\text { Economic } \\
\text { Security }\end{array}$ & 0.12875 \\
\hline 13 & $\begin{array}{l}\text { Low Income } \\
\text { Buffer }\end{array}$ & 0.12401 \\
\hline 17 & $\begin{array}{c}\text { Elderly \& } \\
\text { Disabled } \\
\text { Population } \\
\text { Connectivity }\end{array}$ & 0.11872 \\
\hline 18 & $\begin{array}{l}\text { Emergency } \\
\text { Mobility }\end{array}$ & 0.09271 \\
\hline 16 & $\begin{array}{l}\text { Household } \\
\text { Food Security }\end{array}$ & 0.08125 \\
\hline 19 & $\begin{array}{c}\text { Place } \\
\text { Attachment }\end{array}$ & 0.07998 \\
\hline I1 & $\begin{array}{c}\text { Civic } \\
\text { Participation }\end{array}$ & 0.06694 \\
\hline
\end{tabular}

B.

\begin{tabular}{|c|c|c|}
\hline \multicolumn{3}{|c|}{ NEIGHBORHOOD / COMMUNITY } \\
\hline ID & Indicator & Weight \\
\hline N6 & Income Equity & 0.1295534 \\
\hline N7 & Racial Diversity & 0.1244979 \\
\hline N1 & $\begin{array}{c}\text { Community } \\
\text { Involvement - } \\
\text { Residential }\end{array}$ & 0.1040401 \\
\hline N9 & $\begin{array}{c}\text { Occupational } \\
\text { Diversity }\end{array}$ & 0.102836 \\
\hline N10 & $\begin{array}{c}\text { Business Size } \\
\text { Diversity }\end{array}$ & 0.0977915 \\
\hline N3 & $\begin{array}{c}\text { Community } \\
\text { Involvement - } \\
\text { Business }\end{array}$ & 0.0946334 \\
\hline N11 & $\begin{array}{c}\text { Innovative } \\
\text { Potential }\end{array}$ & 0.0855975 \\
\hline N2 & $\begin{array}{c}\text { Political } \\
\text { Engagement }\end{array}$ & 0.0739333 \\
\hline Age Attachment & 0.0527448 \\
\hline Identity & 0.0494823 \\
\hline N8 & $\begin{array}{c}\text { Community } \\
\text { N }\end{array}$ & 0.0848898 \\
\hline
\end{tabular}

C. GOVERNMENT / INSTITUTIONS

\begin{tabular}{|c|c|c|}
\hline ID & Indicator & Weight \\
\hline G7 & $\begin{array}{l}\text { Emergency Health \& } \\
\text { Medical Mitigation }\end{array}$ & 0.1603293 \\
\hline G5 & $\begin{array}{l}\text { Gender-Based } \\
\text { Emergency } \\
\text { Mitigation } \\
\end{array}$ & 0.128999 \\
\hline G8 & $\begin{array}{l}\text { Non-Profit Services } \\
\text { Representation }\end{array}$ & 0.1269411 \\
\hline G3 & $\begin{array}{c}\text { Extreme } \\
\text { Temperature } \\
\text { Mitigation Effort } \\
\end{array}$ & 0.1245115 \\
\hline G1 & $\begin{array}{l}\text { Elected Official } \\
\text { Connectivity }\end{array}$ & 0.1094313 \\
\hline G4 & $\begin{array}{l}\text { Emergency Food } \\
\text { Mitigation Effort }\end{array}$ & 0.1042028 \\
\hline G6 & $\begin{array}{l}\text { Government- } \\
\text { Maintained Multi- } \\
\text { Purpose Space }\end{array}$ & 0.0827076 \\
\hline G2 & $\begin{array}{c}\text { Economic } \\
\text { Development Effort }\end{array}$ & 0.0819812 \\
\hline G9 & Capital Development & 0.0808962 \\
\hline
\end{tabular}

Figure 15. Distribution and Standard Deviation Categories of Resilience Index

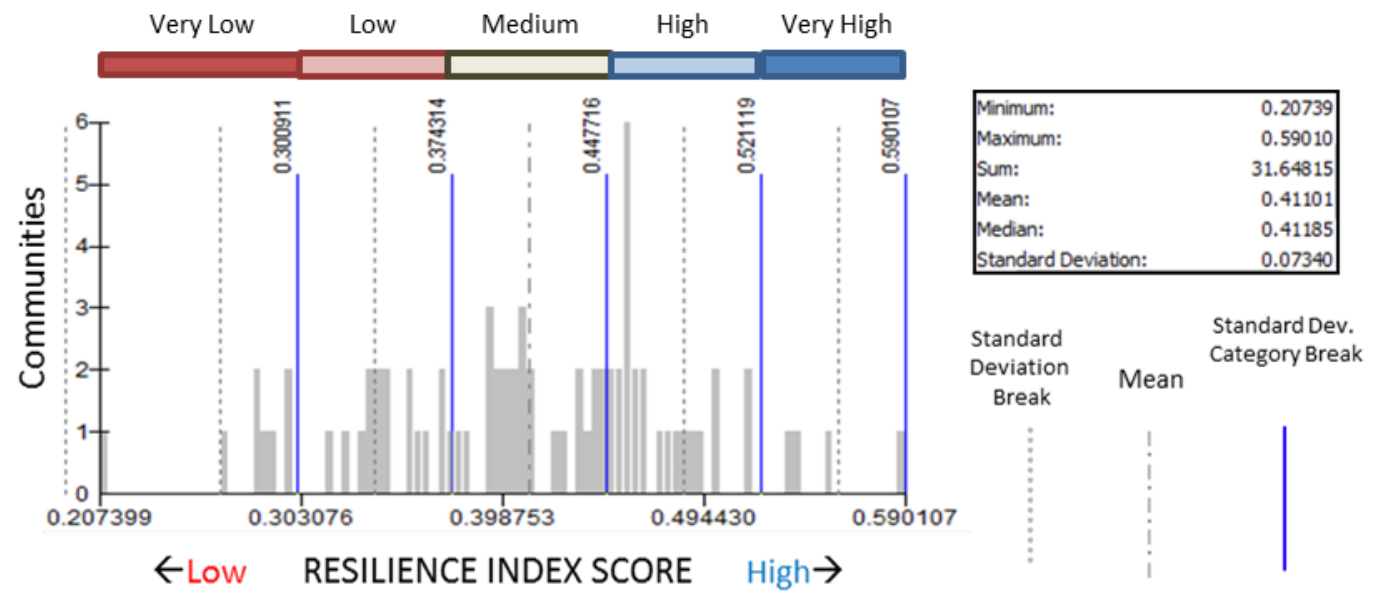


Figure 16. Map of Neighborhood Absolute Resilience

\section{Absolute Resilience of St. Louis Neighborhoods}

1. Carondelet
2. Patch

2. Holy Hills

4. Boulevard Heights

5. Bevo Mil

6. Pinceton Heigts

7. South Hampton

9. Linderwod Pak

10. Elendale

11. Cition Heights

12. The Hill

13. Southwest Garden

14. Nom Harntion

15. Tower Grove South
16. Dutchtown

17. Duchiown

18. Marne Vula

19. Gravis Park

20. Koociubio

21. Soluard

22. Berton Park

24. Fox Park

25. Tower Grove East

26. Compion Heigh

22. Mckiee Toun

29. Tittany

30. Benton Park West

31. The Gate Distrid

32. Lafoyete Square

3.. Peascody Darst Webce

35. Dasalle Park

36. Dowrtown West

36. Dowrtown West
37. Motionn

38. Cenral West End

39. Forest Park Soush Eart

40. Kings Oak

41. Chevenham

42. Clayton-Tam

4 4. Heponte

45. Wydown Strike

46. Sinker Debaliviere

47. Debalviere Place

48. West End

49. Vistation Park

50. Wells Goodtellow

51. Acadeny

52. Kngsway West

54. Lewis Place

55. Kingaway East

56. Grester Vile

57. The Vile

58. Vanseventer

59. Jeff Vandertou

60. Se Lous Place

62 columitus savare

63. Old Noth S Lous

64. Near Noreh Fiverfont

65. Hyde Park

66. College Hill

67. Fairgound Neigrbohocod

68. OFalion

70. Mark Twain L-70 Inductrial

71. Mark Twain

2. Wainut Park Ea

74. Baden

5. Fivervin

76. Wainut Pank West

Covenant Blu Grand Certer

78. Hamilon Heights

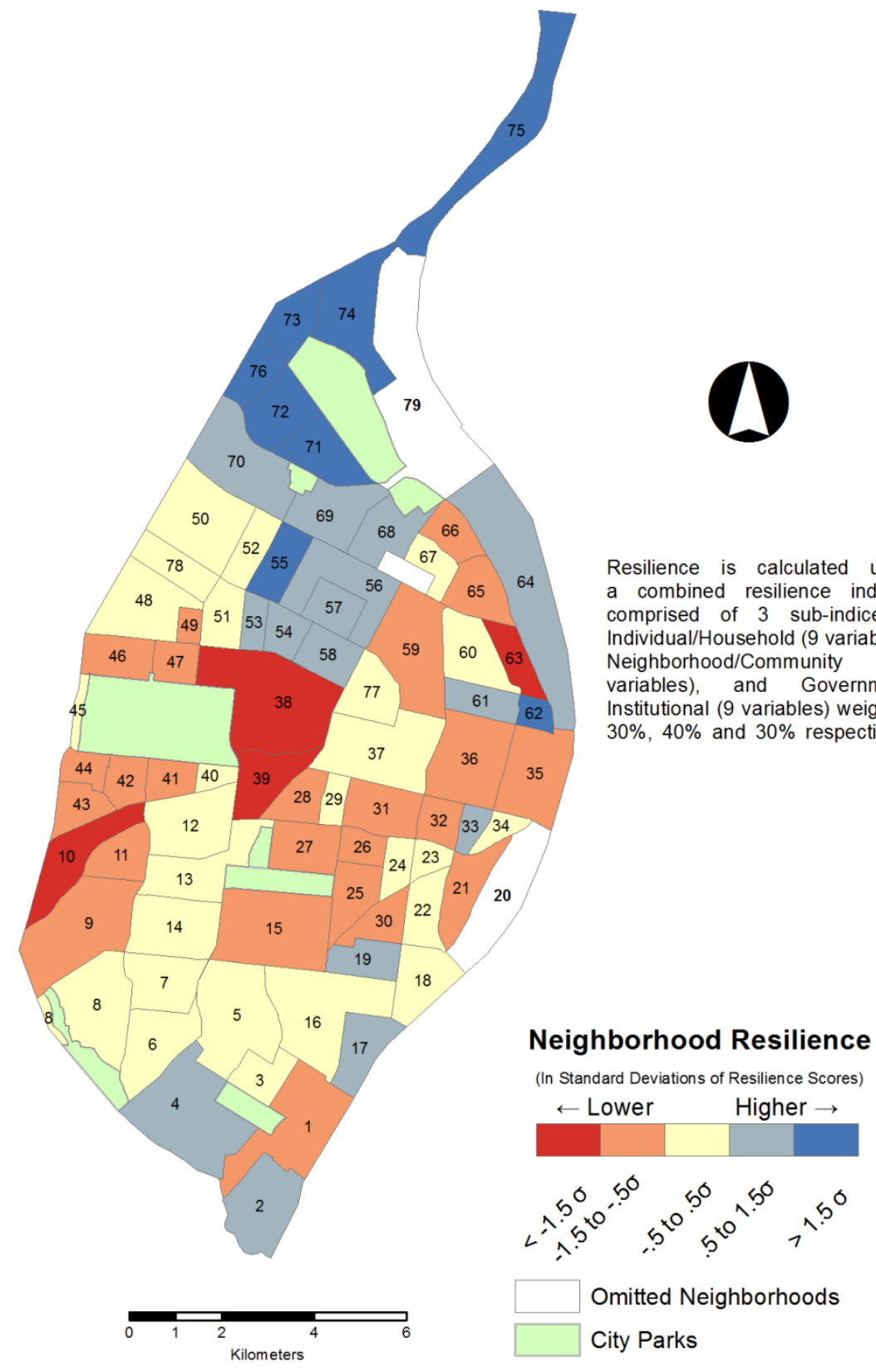




\subsection{Confusion Matrix and Relative Resilience Classifications}

Table 14. Neighborhood Classified by Vulnerability/Resilience

\begin{tabular}{|c|c|c|c|c|c|c|}
\hline & \multicolumn{5}{|c|}{ RESILIENCE } \\
\hline & & Very Low & Low & Medium & High & Very High \\
\hline \multirow{5}{*}{ 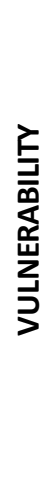 } & Very High & NONE & 2,61 & $18,37,60$ & 59 & NONE \\
\hline & High & $62,71,75$ & $17,19,64$ & $\begin{array}{c}16,22,24,29,50 \\
67,77\end{array}$ & $\begin{array}{c}1,28,30,32,36 \\
65\end{array}$ & NONE \\
\hline & Medium & $55,72,54$ & $\begin{array}{c}33,54,56,57,58 \\
68,69,70\end{array}$ & $\begin{array}{c}3,5,23,34,40,48 \\
52,78\end{array}$ & $\begin{array}{c}15,21,25,27,31 \\
35,41,49,66\end{array}$ & 39,63 \\
\hline & Low & 73,76 & 43 & 12,51 & $\begin{array}{c}4,11,26,42,46 \\
47\end{array}$ & 10,38 \\
\hline & Very Low & NONE & NONE & $6,7,8,13,14,45$ & $9,43,44$ & NONE \\
\hline
\end{tabular}

NOTE: See Table 5 in Chapter 3 for classification labels for relative resilience

Figure 17. Distribution of Neighborhoods in Relative Resilience Categories

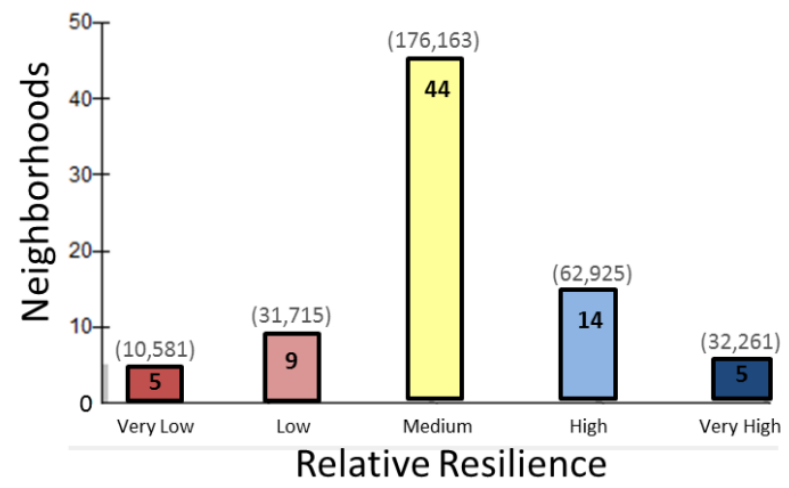

Total civilian population of neighborhoods in each category noted in parentheses (Source: ACS 2011) 
Figure 18. Map of Neighborhood Relative Resilience (Pre-Validation Classification)

\section{Relative Resilience of St. Louis Neighborhoods}

1. Carondelet

2. Pach

4. Boulevard Heights

5. Bevo Mil

7. South Hampoton

8 . Se Lous Hils

9. Undernwod Park

10. Elendale

11. Citon Heigh

13. Southwest Garden

15. Nomin Hamplon

16. Dutchlown

17. Mount Pleacan

18. Marne Vlia

19. Gravis Park

20. Kobousko

21. Solusrd

22. Benton Park

23. Mckinley Heist
24. Fox Park

25. Tower Grove East

27. Shaw

28. McRiee Town

30. Berton Park West

31. The Gate District

32 Latoyecte Square

34. Lasalle Park

35. Dowitown

36. Dowrtown West

37. Motioun Wert En

38. Conzad West End

99. Forest Park Souch Ea

41. Chelienham

42 Cianon-Tamm

43. Franz Park

4 . Hipointe

45. Wydown Siniker

45. Sinker Debaliviere

47. Debalivere Plase

49. Vest End

50. Wells Goodtellow

51. Acadeny

52. Kngsway West

53. Fountain Pak

54. Lewis Place

55. Kingoway Eact

56. Greater Vile

58. Vanseventer

59. veff Vandertou

60. Se Lous Place

61. Carr Square

62 Columbus Square

63. Old Noth SL Lous

64. Near Noth Rivetront

.

67. Fairground Neigrborhood

68 OFalion

. Perrose

70. Mark Twain 170 Industria

71. Mark Twain

72. Wanut Pank East

73. Norn Porte

75. Basen

76. Walnut Pank West

77. Covenant Blu Grand Center

78. Hamilon Heights

79. Norn Riverfont

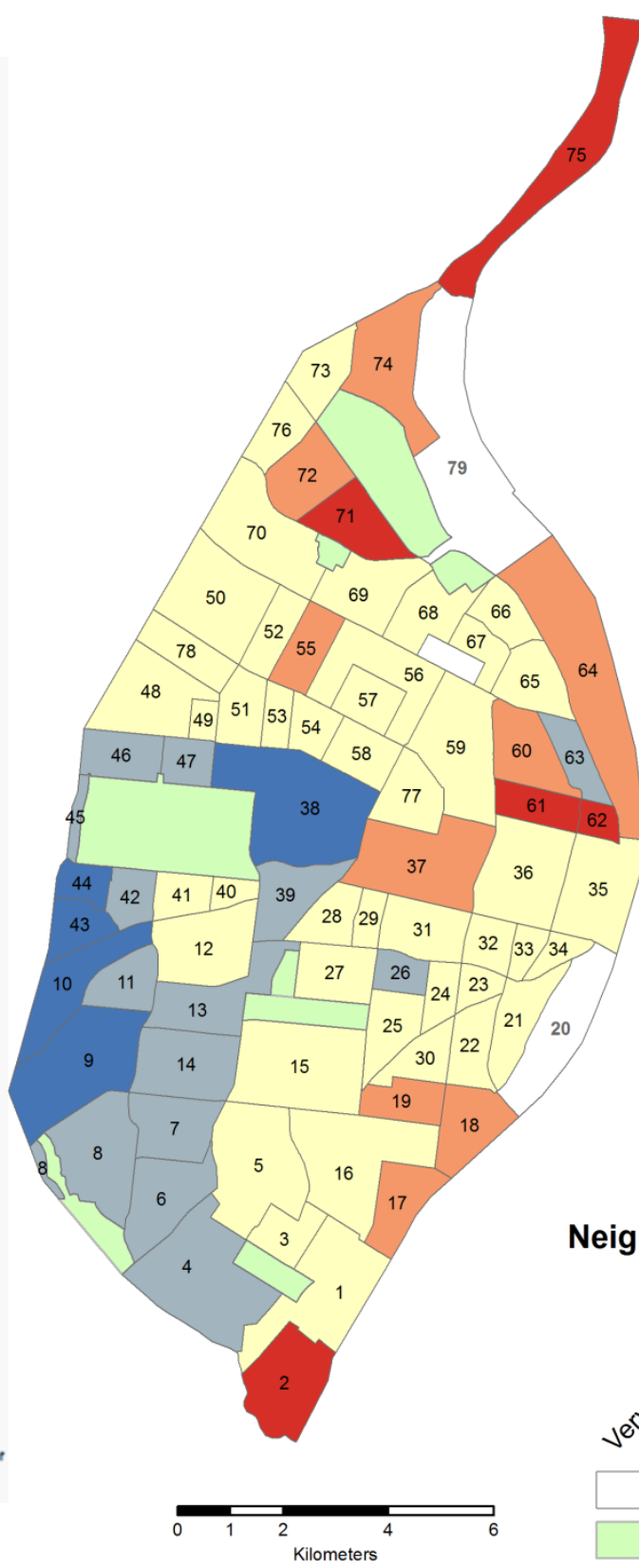

Relative Resilience is defined

as the amount of community protection against the effects

of disaster (as measured by a

29-variable Resilience Index)

compared to the level of threat

posed to the community by

environmental and socioeconomic

threats (as measured by a

13-variable Vulnerability Index).

Population of Low-Resilience Neighborhoods

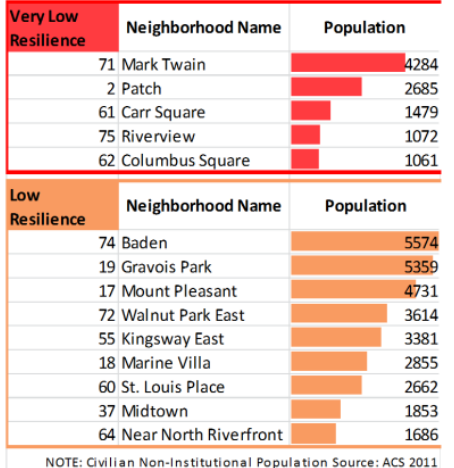

NOTE: Civilian Non-Instituro

Neighborhood Relative Resilience
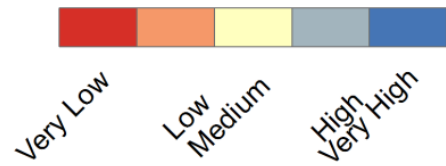

Omitted Neighborhoods

City Parks 


\subsection{DEMATEL Results and Indicator Influence Diagram}

\section{Table 15. DEMATEL Matrix N (Normalized Direct Relation Matrix)}

\begin{tabular}{|c|c|c|c|c|c|c|c|c|c|c|c|c|c|c|c|c|c|c|c|c|c|c|c|c|c|c|c|c|c|}
\hline ID & 11 & 12 & 13 & 14 & 15 & 16 & 17 & 18 & 19 & N1 & N2 & N3 & N4 & N5 & N6 & N7 & N8 & N9 & N10 & N11 & G1 & G2 & G3 & G4 & G5 & G6 & G7 & G8 & G9 \\
\hline I1 & 0 & 0.2 & 0.4 & 0.4 & 0.2 & \begin{tabular}{l|}
0.2 \\
\end{tabular} & 0.4 & \begin{tabular}{l|l}
0 \\
\end{tabular} & \begin{tabular}{|l|}
0.2 \\
\end{tabular} & \begin{tabular}{|l|}
0.4 \\
\end{tabular} & \begin{tabular}{l|l}
0.2 \\
\end{tabular} & \begin{tabular}{l|}
0.2 \\
\end{tabular} & \begin{tabular}{|l|}
0.2 \\
\end{tabular} & \begin{tabular}{|l|}
0.4 \\
\end{tabular} & \begin{tabular}{l|l}
0.2 \\
\end{tabular} & \begin{tabular}{l|}
0.2 \\
\end{tabular} & 0.2 & \begin{tabular}{|l|}
0.4 \\
\end{tabular} & \begin{tabular}{l|}
0.2 \\
\end{tabular} & \begin{tabular}{|l|}
0.4 \\
\end{tabular} & 0.2 & \begin{tabular}{|l|}
0.6 \\
\end{tabular} & \begin{tabular}{l|l}
0.6 \\
\end{tabular} & \begin{tabular}{|c|}
0.6 \\
\end{tabular} & 0.6 & \begin{tabular}{l|}
0.2 \\
\end{tabular} & \begin{tabular}{l|l|}
0.6 \\
\end{tabular} & \begin{tabular}{|c|}
0.6 \\
\end{tabular} & $\overline{0.4}$ \\
\hline 12 & 1 & 0 & 0.8 & 1 & 1 & \begin{tabular}{l|l}
0.8 \\
\end{tabular} & 0.6 & 1 & \begin{tabular}{l|l}
0.6 \\
\end{tabular} & \begin{tabular}{l|l}
0.8 \\
\end{tabular} & \begin{tabular}{|l|}
0.8 \\
\end{tabular} & 0.6 & 0.2 & \begin{tabular}{|c|}
0.8 \\
\end{tabular} & 0.8 & 0.6 & 0.4 & \begin{tabular}{|l|}
0.8 \\
\end{tabular} & 1 & \begin{tabular}{l|l}
0.8 \\
\end{tabular} & 0.6 & \begin{tabular}{l|l}
0.6 \\
\end{tabular} & \begin{tabular}{l|l}
0.4 \\
\end{tabular} & 0.4 & 0.4 & 0.4 & $\begin{array}{ll}0.4 \\
\end{array}$ & 0.4 & 0.8 \\
\hline 13 & 0.6 & 0.6 & 0 & 0.6 & \begin{tabular}{|l|}
0.6 \\
\end{tabular} & $\begin{array}{ll}0.2 \\
\end{array}$ & 0.4 & 0.4 & 0.4 & 0.4 & 0.2 & 0.4 & 0.2 & 0.4 & \begin{tabular}{l|l}
0.4 \\
\end{tabular} & 0.4 & 0.2 & 0.4 & 0.4 & 0.4 & 0.4 & \begin{tabular}{l|l}
0.6 \\
\end{tabular} & \begin{tabular}{l|l}
0.4 \\
\end{tabular} & 0.4 & \begin{tabular}{l|l}
0.4 \\
\end{tabular} & \begin{tabular}{l|}
0.2 \\
\end{tabular} & 0.4 & 0.8 & 0.4 \\
\hline 14 & 0.4 & 0.4 & 0.6 & 0 & 0.4 & $\begin{array}{ll}0.2 \\
\end{array}$ & 0.4 & \begin{tabular}{l|l}
0.2 \\
\end{tabular} & 0.2 & $\begin{array}{ll}0.2 \\
\end{array}$ & $\begin{array}{ll}0.2 \\
\end{array}$ & $\begin{array}{ll}0.2 \\
\end{array}$ & 0.2 & 0.4 & \begin{tabular}{ll|} 
\\
\end{tabular} & \begin{tabular}{l|}
0.2 \\
\end{tabular} & 0.2 & 0.4 & \begin{tabular}{l|}
0.2 \\
\end{tabular} & 0.2 & 0.4 & 0.4 & \begin{tabular}{l|l}
0.4 \\
\end{tabular} & 0.4 & 0.4 & \begin{tabular}{l|l}
0.2 \\
\end{tabular} & 0.4 & 0.6 & 0.4 \\
\hline 15 & 0.6 & 0.6 & 0.4 & 0.8 & 0 & \begin{tabular}{l|}
0.8 \\
\end{tabular} & $\begin{array}{ll}0.6 \\
\end{array}$ & 0.6 & \begin{tabular}{l|l}
0.6 \\
\end{tabular} & 0.6 & \begin{tabular}{l|l}
0.6 \\
\end{tabular} & 0.4 & 0.2 & 0.4 & 0.6 & 0.4 & 0.4 & \begin{tabular}{l|l}
0.4 \\
\end{tabular} & 0.4 & \begin{tabular}{l|l}
0.4 \\
\end{tabular} & \begin{tabular}{l|l}
0.4 \\
\end{tabular} & 0.4 & \begin{tabular}{l|l}
0.4 \\
\end{tabular} & \begin{tabular}{l|l}
0.4 \\
\end{tabular} & 0.4 & 0.2 & 0.4 & 0.6 & 0.4 \\
\hline 16 & \begin{tabular}{l|l|}
0.4 \\
\end{tabular} & 0.2 & 0.2 & 0.4 & \begin{tabular}{|l|}
0.4 \\
\end{tabular} & 0 & 0.2 & \begin{tabular}{l|l}
0.2 \\
\end{tabular} & \begin{tabular}{l|l}
0.6 \\
\end{tabular} & 0.4 & \begin{tabular}{|l|}
0.8 \\
\end{tabular} & 0.2 & 0.2 & 0.2 & 0.2 & 0.2 & 0.2 & \begin{tabular}{l|}
0.2 \\
\end{tabular} & \begin{tabular}{l|l}
0.2 \\
\end{tabular} & \begin{tabular}{l|l}
0.4 \\
\end{tabular} & \begin{tabular}{l|l}
0.2 \\
\end{tabular} & \begin{tabular}{l|l}
0.2 \\
\end{tabular} & 0.2 & \begin{tabular}{l|}
0.2 \\
\end{tabular} & 0.2 & 0.2 & 0.2 & 0.4 & 0.4 \\
\hline 17 & 0.6 & 0.2 & 0.2 & 0.4 & 0.4 & 0.2 & 0 & \begin{tabular}{l|l}
0.2 \\
\end{tabular} & \begin{tabular}{l|l}
0.4 \\
\end{tabular} & 0.4 & \begin{tabular}{|l|}
0.4 \\
\end{tabular} & 0.2 & 0.2 & 0.4 & 0.2 & 0.2 & 0.4 & 0.2 & \begin{tabular}{l|l}
0.2 \\
\end{tabular} & \begin{tabular}{l|l}
0.2 \\
\end{tabular} & 0.2 & \begin{tabular}{l|l}
0.4 \\
\end{tabular} & \begin{tabular}{l|l}
0.4 \\
\end{tabular} & 0.4 & 0.2 & \begin{tabular}{l|l}
0.2 \\
\end{tabular} & 0.4 & \begin{tabular}{l|l}
0.4 \\
\end{tabular} & 0.2 \\
\hline 18 & 0.2 & $\begin{array}{ll}0.6 \\
\end{array}$ & \begin{tabular}{l|l}
0.4 \\
\end{tabular} & \begin{tabular}{|l|}
0.4 \\
\end{tabular} & 0.4 & $\begin{array}{ll}0.4 \\
\end{array}$ & 0.2 & 0 & 0.2 & $\begin{array}{ll}0.2 \\
\end{array}$ & 0.2 & $\begin{array}{ll}0.2 \\
\end{array}$ & 4 & 0.2 & 0.2 & 0.2 & 0.2 & 0.2 & \begin{tabular}{l|l}
0.4 \\
\end{tabular} & 0.2 & 0.2 & \begin{tabular}{l|l}
0.2 \\
\end{tabular} & 0.2 & \begin{tabular}{l|l}
0.2 \\
\end{tabular} & 0.2 & 0 & 0.4 & \begin{tabular}{|l|}
0.4 \\
\end{tabular} & 0.4 \\
\hline 19 & 0.6 & 0.4 & 0.4 & 0.6 & 0.6 & 0.6 & \begin{tabular}{l|l}
0.6 \\
\end{tabular} & 0.2 & 0 & 0.6 & \begin{tabular}{|l|}
0.4 \\
\end{tabular} & 0.4 & \begin{tabular}{l|l}
0.4 \\
\end{tabular} & 0.6 & \begin{tabular}{l|l}
0.2 \\
\end{tabular} & 0.2 & 0.4 & 0.2 & 0.2 & 0.4 & \begin{tabular}{l|l}
0.4 \\
\end{tabular} & 0.4 & 0.2 & \begin{tabular}{l|l}
0.2 \\
\end{tabular} & 0.2 & $\begin{array}{ll}0.4 \\
\end{array}$ & 0.2 & \begin{tabular}{l|}
0.2 \\
\end{tabular} & 0.4 \\
\hline N1 & 0.6 & 0.4 & 0.4 & 0.4 & \begin{tabular}{l|}
0.2 \\
\end{tabular} & $\begin{array}{ll}0.2 \\
\end{array}$ & 0.6 & \begin{tabular}{l|l}
0.2 \\
\end{tabular} & \begin{tabular}{l|l}
0.6 \\
\end{tabular} & 0 & \begin{tabular}{|l|}
0.6 \\
\end{tabular} & 1 & 0.4 & 0.8 & \begin{tabular}{l|l}
0.4 \\
\end{tabular} & 0.4 & 0.4 & 0.4 & 0.4 & 0.4 & 1 & \begin{tabular}{l|l}
0.8 \\
\end{tabular} & 0.4 & 0.4 & 0.4 & 0.4 & 0.4 & 0.6 & 0.4 \\
\hline N2 & 0.4 & 0.2 & 0.2 & 0.2 & 0.2 & \begin{tabular}{|l|}
0.8 \\
\end{tabular} & 0.4 & \begin{tabular}{l|l}
0.2 \\
\end{tabular} & \begin{tabular}{l|l}
0.6 \\
\end{tabular} & 0.6 & 0 & 0.4 & 0.2 & 0.4 & 0.2 & 0.2 & 0.2 & 0.2 & \begin{tabular}{l|l}
0.2 \\
\end{tabular} & 0.2 & 0.4 & 0.4 & 0.2 & \begin{tabular}{l|l}
0.2 \\
\end{tabular} & 0.2 & 0.4 & 0.2 & 0.4 & 0.2 \\
\hline N3 & 0.2 & 0.2 & 0.2 & 0.2 & 0.2 & $\begin{array}{ll}0.2 \\
\end{array}$ & 0.2 & \begin{tabular}{l|l}
0.2 \\
\end{tabular} & 0.4 & \begin{tabular}{|l|}
0.8 \\
\end{tabular} & \begin{tabular}{|l|}
0.4 \\
\end{tabular} & 0 & 0.4 & 0.4 & 0.4 & 0.4 & 0.2 & 0.4 & \begin{tabular}{l|l}
0.6 \\
\end{tabular} & 0.4 & 0.6 & \begin{tabular}{l|l}
0.8 \\
\end{tabular} & 0.8 & \begin{tabular}{l|l}
0.2 \\
\end{tabular} & 0.2 & 0.4 & 0.2 & 0.4 & 0.6 \\
\hline N4 & 0.4 & 0.2 & 0.2 & 0.2 & 0.2 & $\begin{array}{ll}0.2 \\
\end{array}$ & 0.2 & \begin{tabular}{l|l}
0.2 \\
\end{tabular} & 0.4 & 0.6 & \begin{tabular}{|c|}
0.4 \\
\end{tabular} & 0.4 & 0 & \begin{tabular}{l|}
0.2 \\
\end{tabular} & 0.2 & 0.2 & 0.2 & 0.2 & \begin{tabular}{l|l}
0.2 \\
\end{tabular} & 0.2 & 0.2 & \begin{tabular}{l|l}
0.6 \\
\end{tabular} & 0.2 & \begin{tabular}{l|l}
0.2 \\
\end{tabular} & 0.2 & 0.4 & 0.2 & $\begin{array}{ll}0.2 \\
\end{array}$ & 0.2 \\
\hline N5 & \begin{tabular}{l|l}
0.8 \\
\end{tabular} & 0.4 & 0.4 & 0.2 & 0.2 & $\begin{array}{ll}0.2 \\
\end{array}$ & 0.4 & \begin{tabular}{l|l}
0.2 \\
\end{tabular} & 0.4 & 0.4 & 0.2 & 0.4 & 0.4 & 0 & \begin{tabular}{l|l}
0.2 \\
\end{tabular} & 0.2 & $\begin{array}{ll}0.2 \\
\end{array}$ & 0.2 & \begin{tabular}{l|l}
0.2 \\
\end{tabular} & 0.2 & 1 & \begin{tabular}{l|l}
0.6 \\
\end{tabular} & 0.2 & 0.2 & 0.2 & $\begin{array}{ll}0.2 \\
\end{array}$ & 0.2 & 0.4 & 0.2 \\
\hline N6 & 0.4 & 0.6 & 0.6 & 0.6 & \begin{tabular}{l|}
0.6 \\
\end{tabular} & 0.4 & 0.2 & \begin{tabular}{l|l}
0.2 \\
\end{tabular} & 0.4 & \begin{tabular}{l|l}
0.6 \\
\end{tabular} & \begin{tabular}{l|l}
0.6 \\
\end{tabular} & 0.6 & 0.2 & 0.6 & 0 & & $\begin{array}{ll}0.6 \\
\end{array}$ & 1 & \begin{tabular}{l|l}
0.8 \\
\end{tabular} & \begin{tabular}{l|l}
0.8 \\
\end{tabular} & \begin{tabular}{l|l}
0.6 \\
\end{tabular} & \begin{tabular}{|l|l|}
0.6 \\
\end{tabular} & 0.6 & \begin{tabular}{l|l}
0.6 \\
\end{tabular} & 0.6 & $\begin{array}{ll}0.2 \\
\end{array}$ & 0.4 & \begin{tabular}{l|l}
0.8 \\
\end{tabular} & 0.8 \\
\hline N7 & 0.6 & 0.6 & 0.6 & 0.6 & 0.6 & 0.4 & 0.4 & 0.4 & \begin{tabular}{l|l}
0.6 \\
\end{tabular} & 0.6 & \begin{tabular}{l|l}
0.6 \\
\end{tabular} & 0.4 & 0.2 & 0.6 & 0.8 & 0 & 0.4 & \begin{tabular}{|l|l|} 
\\
\end{tabular} & \begin{tabular}{l|l}
0.6 \\
\end{tabular} & \begin{tabular}{l|l}
0.6 \\
\end{tabular} & 0.6 & \begin{tabular}{|l|l|}
0.6 \\
\end{tabular} & 0.4 & 0.4 & 0.4 & 0.2 & 0.4 & \begin{tabular}{l|l}
0.8 \\
\end{tabular} & 0.6 \\
\hline N8 & 0.4 & 0.4 & 0.2 & 0.4 & 0.4 & $\begin{array}{ll}0.2 \\
\end{array}$ & 0.6 & $\begin{array}{ll}0.2 \\
\end{array}$ & 0.4 & 0.4 & 0.4 & 0.4 & 0.2 & 0.4 & 0.4 & \begin{tabular}{l|l}
0.2 \\
\end{tabular} & 0 & \begin{tabular}{l|l}
0.4 \\
\end{tabular} & 0.4 & 0.4 & \begin{tabular}{l|l}
0.4 \\
\end{tabular} & 0.2 & 0.4 & \begin{tabular}{l|l} 
\\
\end{tabular} & 0.4 & 0.2 & 0.4 & 0.6 & 0.4 \\
\hline N9 & \begin{tabular}{l|l}
0.2 \\
\end{tabular} & 0.4 & 0.4 & 0.6 & 0.6 & 0.4 & 0.4 & 0.2 & 0.4 & 0.4 & \begin{tabular}{l|l}
0.4 \\
\end{tabular} & 0.4 & 0.2 & 0.2 & 0.8 & 0.6 & $\begin{array}{ll}0.6 \\
\end{array}$ & 0 & \begin{tabular}{l|l}
0.8 \\
\end{tabular} & 0.6 & \begin{tabular}{l|l}
0.6 \\
\end{tabular} & \begin{tabular}{l|l}
0.6 \\
\end{tabular} & 0.2 & 0.2 & 0.2 & \begin{tabular}{l|}
0.2 \\
\end{tabular} & \begin{tabular}{l|l}
0.2 \\
\end{tabular} & 0.4 & 0.6 \\
\hline N10 & \begin{tabular}{l|}
0.2 \\
\end{tabular} & \begin{tabular}{l|}
0.2 \\
\end{tabular} & 0.2 & 0.2 & 0.4 & 0.4 & 0.2 & \begin{tabular}{l|l}
0.2 \\
\end{tabular} & \begin{tabular}{l|l}
0.6 \\
\end{tabular} & 0.4 & 0.2 & 0.8 & 0.2 & 0.2 & 0.4 & 0.4 & 0.4 & \begin{tabular}{l|l}
0.4 \\
\end{tabular} & 0 & \begin{tabular}{l|l}
0.4 \\
\end{tabular} & \begin{tabular}{l|l}
0.4 \\
\end{tabular} & \begin{tabular}{l|l|}
0.8 & \\
\end{tabular} & 0.2 & 0.2 & 0. & \begin{tabular}{l|}
0.2 \\
\end{tabular} & 0.2 & $\begin{array}{ll}0.4 \\
\end{array}$ & 0.6 \\
\hline N11 & \begin{tabular}{l|}
0.4 \\
\end{tabular} & 0.4 & 0.2 & 0.4 & 0.4 & 0.2 & 0.2 & 0.4 & \begin{tabular}{l|l}
0.2 \\
\end{tabular} & 0.2 & 0.2 & 0.4 & 0.2 & 0.4 & 0.6 & 0.4 & 0.4 & \begin{tabular}{l|l}
0.6 \\
\end{tabular} & 0.4 & 0 & \begin{tabular}{l|l}
0.4 \\
\end{tabular} & \begin{tabular}{l|l}
0.4 \\
\end{tabular} & 0.2 & 0.2 & 0.2 & \begin{tabular}{l|}
0.2 \\
\end{tabular} & 0.2 & \begin{tabular}{l|l}
0.2 \\
\end{tabular} & 0.4 \\
\hline G1 & \begin{tabular}{l|}
0.4 \\
\end{tabular} & \begin{tabular}{l|}
0.2 \\
\end{tabular} & 0.4 & 0.2 & 0.2 & 0.2 & 0.6 & $\begin{array}{ll}0.2 \\
\end{array}$ & \begin{tabular}{l|l}
0.4 \\
\end{tabular} & $\begin{array}{ll}0.8 \\
\end{array}$ & \begin{tabular}{|l|}
0.4 \\
\end{tabular} & 0.6 & 0.4 & 0.6 & 0.2 & \begin{tabular}{l|l|}
0.4 \\
\end{tabular} & 0.4 & \begin{tabular}{l|l}
0.4 \\
\end{tabular} & 0.4 & \begin{tabular}{l|l}
0.2 \\
\end{tabular} & 0 & \begin{tabular}{l|l|}
0.8 & \\
\end{tabular} & 0.2 & 0.2 & 0.2 & \begin{tabular}{l|}
0.2 \\
\end{tabular} & 0.2 & $\begin{array}{ll}0.4 \\
\end{array}$ & 0.2 \\
\hline G2 & \begin{tabular}{l|}
0.2 \\
\end{tabular} & $\begin{array}{ll}0.2 \\
\end{array}$ & 0.2 & \begin{tabular}{l|}
0.2 \\
\end{tabular} & \begin{tabular}{l|l}
0.2 \\
\end{tabular} & 0.2 & \begin{tabular}{l|l}
0.2 \\
\end{tabular} & $\begin{array}{ll}0.2 \\
\end{array}$ & \begin{tabular}{l|l}
0.4 \\
\end{tabular} & 0.4 & \begin{tabular}{|l|}
0.4 \\
\end{tabular} & 0.4 & \begin{tabular}{l|}
0.2 \\
\end{tabular} & \begin{tabular}{l|}
0.2 \\
\end{tabular} & \begin{tabular}{l|l}
0.2 \\
\end{tabular} & \begin{tabular}{l|l}
0.2 \\
\end{tabular} & \begin{tabular}{l|l}
0.2 \\
\end{tabular} & 0.2 & $\begin{array}{ll}0.2 \\
\end{array}$ & \begin{tabular}{l|l}
0.2 \\
\end{tabular} & \begin{tabular}{l|l}
0.6 \\
\end{tabular} & \begin{tabular}{l|l}
0 \\
\end{tabular} & 0.2 & \begin{tabular}{l|l}
0.2 \\
\end{tabular} & 0.2 & \begin{tabular}{l|}
0.2 \\
\end{tabular} & 0.2 & \begin{tabular}{l|l}
0.4 \\
\end{tabular} & 0.4 \\
\hline G3 & $\begin{array}{ll}0.2 \\
\end{array}$ & 0 & 0 & \begin{tabular}{l|}
0.2 \\
\end{tabular} & - & 0 & \begin{tabular}{l|l}
0.8 \\
\end{tabular} & \begin{tabular}{l|l}
0.2 \\
\end{tabular} & \begin{tabular}{l|l}
0.2 \\
\end{tabular} & 0.2 & 0 & 0 & 0 & 0.2 & 0.2 & \begin{tabular}{l|}
0.2 \\
\end{tabular} & \begin{tabular}{l|l}
0.2 \\
\end{tabular} & \begin{tabular}{l|l}
0.2 \\
\end{tabular} & \begin{tabular}{l|l}
0.2 \\
\end{tabular} & \begin{tabular}{l|l}
0.2 \\
\end{tabular} & \begin{tabular}{l|l}
0.2 \\
\end{tabular} & \begin{tabular}{l|l}
0.4 \\
\end{tabular} & 0 & \begin{tabular}{l|l}
0.6 \\
\end{tabular} & 0.6 & \begin{tabular}{l|}
0.2 \\
\end{tabular} & \begin{tabular}{l|l}
0.6 \\
\end{tabular} & \begin{tabular}{l|l}
0.4 \\
\end{tabular} & 0.2 \\
\hline G4 & \begin{tabular}{l|l|}
0.2 \\
\end{tabular} & 0 & 0 & 0 & 0 & 0.2 & $\begin{array}{ll} \\
\end{array}$ & 0 & \begin{tabular}{l|l}
0.2 \\
\end{tabular} & 0.2 & 0.2 & 0 & 이 & 0.2 & 0.2 & \begin{tabular}{l|l}
0.2 \\
\end{tabular} & \begin{tabular}{l|l}
0.2 \\
\end{tabular} & 0.2 & 0.2 & 0.2 & \begin{tabular}{l|l}
0.2 \\
\end{tabular} & \begin{tabular}{l|l}
0.2 \\
\end{tabular} & \begin{tabular}{l|l|}
0.6 \\
\end{tabular} & 0 & 0.6 & 0.2 & 0.6 & 0.4 & 0.2 \\
\hline G5 & 0.2 & 0.2 & 0.2 & \begin{tabular}{l|}
0.2 \\
\end{tabular} & \begin{tabular}{l|l}
0.2 \\
\end{tabular} & 0.2 & 0 & 0 & \begin{tabular}{l|l}
0.2 \\
\end{tabular} & 0.2 & 0.2 & $\begin{array}{ll}0.2 \\
\end{array}$ & 0 & 0.2 & 0.2 & \begin{tabular}{l|}
0.2 \\
\end{tabular} & 0.2 & 0.2 & 0.2 & 0.2 & 0.2 & \begin{tabular}{l|l} 
\\
\end{tabular} & 0.6 & \begin{tabular}{l|l}
0.8 \\
\end{tabular} & 0 & \begin{tabular}{l|}
0.2 \\
\end{tabular} & 0.8 & \begin{tabular}{l|l}
0.6 \\
\end{tabular} & 0.2 \\
\hline G6 & 0.2 & \begin{tabular}{l|l}
0.2 \\
\end{tabular} & \begin{tabular}{l|}
0.2 \\
\end{tabular} & 0 & 0 & 0.2 & 0 & 0 & \begin{tabular}{l|l}
0.4 \\
\end{tabular} & 0.4 & \begin{tabular}{l|l}
0.6 \\
\end{tabular} & 0.2 & 0.4 & 0.2 & 0.2 & \begin{tabular}{l|l}
0.2 \\
\end{tabular} & 0.2 & 0.2 & 0.2 & 0.2 & 0.2 & \begin{tabular}{l|l}
0.4 \\
\end{tabular} & 0.4 & \begin{tabular}{l|l} 
\\
\end{tabular} & \begin{tabular}{l|l}
0.2 \\
\end{tabular} & 0 & \begin{tabular}{l|l}
0.2 \\
\end{tabular} & \begin{tabular}{l|l}
0.2 \\
\end{tabular} & 0.2 \\
\hline G7 & 0.2 & \begin{tabular}{l|}
0.2 \\
\end{tabular} & 0.2 & 0.2 & 0.2 & 0.2 & \begin{tabular}{l|l} 
\\
\end{tabular} & 0.2 & 0.2 & 0.2 & 0.2 & 0.2 & 0 & 0.2 & 0.2 & \begin{tabular}{l|l}
0.2 \\
\end{tabular} & 0.2 & 0.2 & 0.2 & 0.2 & 0.2 & \begin{tabular}{l|l}
0.4 \\
\end{tabular} & 0.6 & \begin{tabular}{l|l}
0.6 \\
\end{tabular} & 0.6 & $\begin{array}{ll}0.2 \\
\end{array}$ & 0 & \begin{tabular}{l|l|}
0.6 \\
\end{tabular} & 0.2 \\
\hline G8 & \begin{tabular}{ll|}
0.4 \\
\end{tabular} & 0.4 & 0.4 & 0.4 & 0.4 & 0.4 & \begin{tabular}{l|l}
0.6 \\
\end{tabular} & 0.2 & 0.2 & 0.4 & 0.2 & $\begin{array}{ll}0.2 \\
\end{array}$ & 0.2 & 0.4 & 0.4 & 0.4 & 0.4 & 0.4 & 0.4 & 0.2 & 0.2 & 0.6 & 0.4 & 0.4 & 0.4 & \begin{tabular}{l|l}
0.2 \\
\end{tabular} & \begin{tabular}{l|l|}
0.4 \\
\end{tabular} & 이 & 0.2 \\
\hline G9 & 이 & 0.2 & 0.2 & 0 & \begin{tabular}{l|l}
0.2 \\
\end{tabular} & 0.2 & $\begin{array}{ll} \\
\end{array}$ & \begin{tabular}{l|}
0.2 \\
\end{tabular} & $\begin{array}{ll}0.2 \\
\end{array}$ & $\begin{array}{ll}0.2 \\
\end{array}$ & 0 & \begin{tabular}{l|}
0.2 \\
\end{tabular} & & 0.2 & \begin{tabular}{l|l}
0.2 \\
\end{tabular} & \begin{tabular}{l|}
0.2 \\
\end{tabular} & \begin{tabular}{l|l} 
\\
\end{tabular} & 0.2 & \begin{tabular}{l|}
0.2 \\
\end{tabular} & \begin{tabular}{l|l}
0.2 \\
\end{tabular} & \begin{tabular}{l|l}
0.2 \\
\end{tabular} & \begin{tabular}{l|l}
0.2 \\
\end{tabular} & \begin{tabular}{l|l}
0.2 \\
\end{tabular} & \begin{tabular}{l|l}
0.2 \\
\end{tabular} & \begin{tabular}{l|l} 
\\
\end{tabular} & 0 & \begin{tabular}{l|l}
0.2 \\
\end{tabular} & \begin{tabular}{l|l}
0.2 \\
\end{tabular} & \\
\hline
\end{tabular}

Table 16. DEMATEL Matrix T (Total Direct-Indirect Matrix)

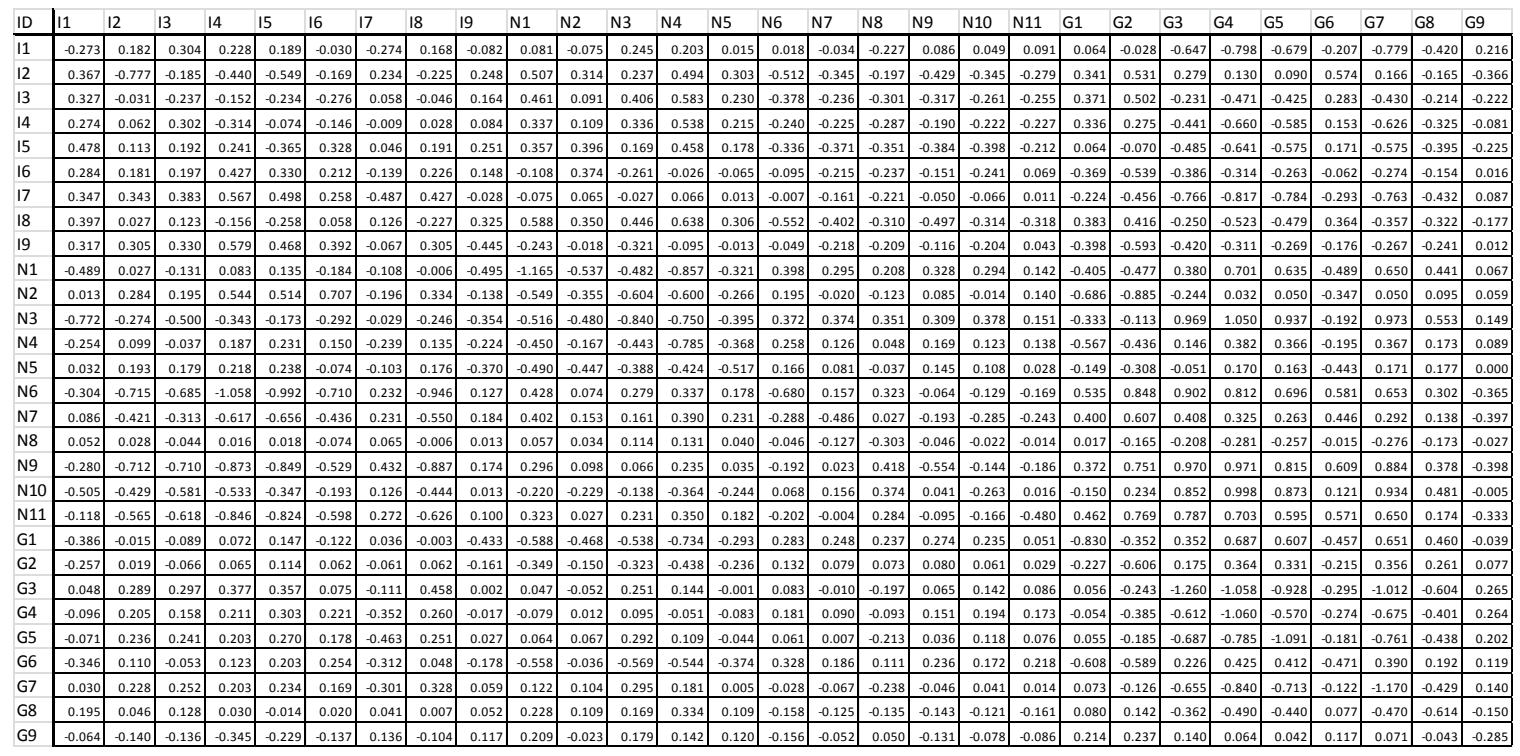


Table 17. Degree of Dispatching Pi, Positional and Relational Values for Resilience Indicators Used in the DEMATEL Analysis

\begin{tabular}{|c|c|c|c|c|}
\hline Indicator ID & Indicator & Pi & $\mathrm{Si+}$ & Si- \\
\hline 11 & Civic Participation & -2.416 & -3.385 & -1.447 \\
\hline 12 & Educational Attainment & -0.171 & -1.275 & 0.933 \\
\hline 13 & Low Income Buffer & -1.242 & -2.346 & -0.139 \\
\hline 14 & Insurance Coverage & -1.603 & -2.907 & -0.299 \\
\hline 15 & Household Economic Security & -1.752 & -3.066 & -0.438 \\
\hline 16 & Household Food Security & -1.434 & -2.319 & -0.549 \\
\hline 17 & Elderly \& Disabled Population Connectivity & -2.591 & -3.808 & -1.374 \\
\hline 18 & Emergency Mobility & -0.594 & -1.505 & 0.318 \\
\hline 19 & Place Attachment & -1.924 & -2.762 & -1.086 \\
\hline N1 & Community Involvement - Residential & -1.362 & -2.246 & -0.478 \\
\hline N2 & Place Attachment & -1.730 & -2.392 & -1.068 \\
\hline N3 & Community Involvement - Business & -0.036 & -0.998 & 0.927 \\
\hline N4 & Community Identity & -0.977 & -1.311 & -0.644 \\
\hline N5 & Political Engagement & -1.557 & -2.617 & -0.498 \\
\hline N6 & Income Equity & 0.648 & -0.729 & 2.025 \\
\hline N7 & Racial Diversity & -0.140 & -1.417 & 1.136 \\
\hline N8 & Age Equity & -1.497 & -2.675 & -0.319 \\
\hline N9 & Occupational Diversity & 1.214 & -0.186 & 2.614 \\
\hline N10 & Business Size Diversity & 0.643 & -0.713 & 1.999 \\
\hline N11 & Innovative Potential & 1.004 & -0.152 & 2.159 \\
\hline G1 & Elected Official Connectivity & -1.007 & -2.183 & 0.170 \\
\hline $\mathrm{G} 2$ & Economic Development Effort & -0.750 & -1.993 & 0.492 \\
\hline G3 & Extreme Temperature Mitigation Effort & -2.730 & -3.849 & -1.612 \\
\hline G4 & Emergency Food Mitigation Effort & -2.286 & -3.520 & -1.051 \\
\hline G5 & Gender-Based Emergency Mitigation & -2.426 & -3.610 & -1.242 \\
\hline G6 & Government-Maintained Multi-Purpose Space & -0.885 & -1.254 & -0.517 \\
\hline G7 & Emergency Health \& Medical Mitigation & -2.256 & -3.433 & -1.080 \\
\hline G8 & Non-Profit Services Representation & -1.617 & -3.161 & -0.072 \\
\hline G9 & Capital Development & -0.173 & -1.484 & 1.138 \\
\hline
\end{tabular}


Figure 19. Causal Diagram of Resilience Indicators Evaluated in DEMATEL Analysis

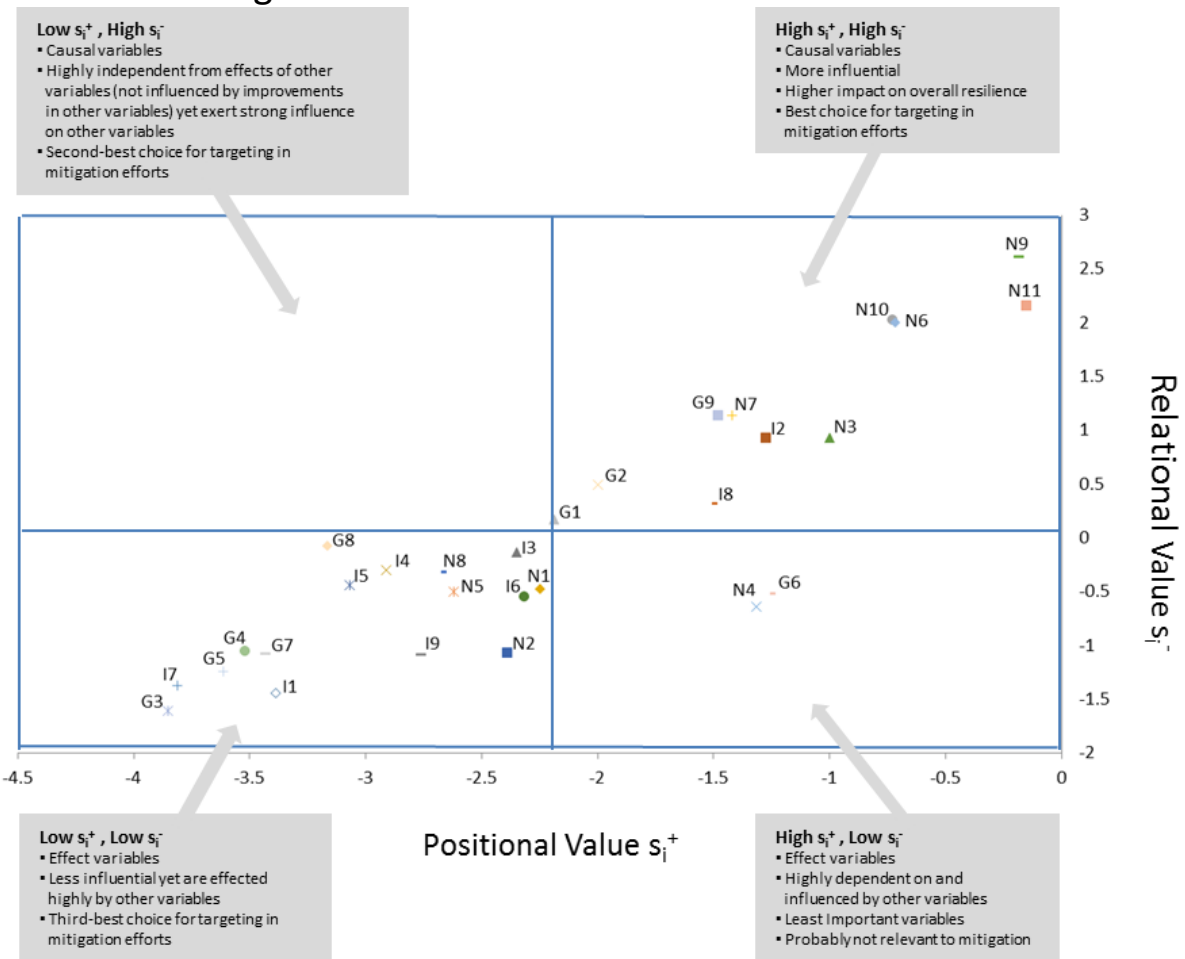




\section{CHAPTER 6. VALIDATION AND DISCUSSION}

\subsection{Internal Validation: Model Sensitivity to Sub-Index Weighting Scheme}

Although it is at first tempting to interpret the results of the relative community resilience analysis as-is, there is reason to believe that these results could be highly dependent on the chosen sub-index weighting scheme (Tate 2012). As noted in Figure 14, the 'Environmental' and 'Socio-Economic' sub-indices of the vulnerability index were weighted $40 \%$ and $60 \%$ respectively. The decision to give higher weight to socioeconomic hazards was guided by the City of St. Louis Hazard Mitigation Plan (EW Gateway 2010a) and the City of St. Louis Public Health Department's publication Understanding Our Needs (STL Health Dept. 2012), both of which suggest that adverse socio-economic conditions within the City tend to attenuate and magnify the effects of extreme events. Likewise, as noted in Figure 16, the 'Individual/Household', 'Neighborhood' and 'Government/Institution' sub-indices of the absolute resilience index were each given a weight of $33 \%$. Less guidance was available in local expert reporting for ranking relative importance of community protections against disaster at the micro/meso/macro levels as represented by these three resilience sub-indices; a balanced weighting scheme was therefore applied. However, it is reasonable that local emergency managers in St. Louis may opt for different weighting schemes in either the vulnerability or resilience indices, and it is therefore important to explore the consequences of alternative weighting scenarios. 
The questions most relevant to disaster mitigation as it relates to sub-index weighting are: 'How do the higher risk classifications of neighborhoods change when different weighting schemes are applied to each index?' and 'Are there neighborhoods that consistently remain in higher risk classifications even when different weighting schemes are applied?' To further explore these questions, the final relative resilience categorizations were recalculated using nine reasonable combinations of alternative weighting schemes for each sub-index of the vulnerability and absolute resilience indices. These weighting schemes are listed below in Table 18, with the original baseline weighting scheme shown as reference.

Table 18. Baseline and Alternative Weighting Schemes Used In Sensitivity Analysis

\begin{tabular}{|c|c|c|c|c|c|c|c|c|c|c|c|}
\hline & & \multicolumn{10}{|c|}{ Baseline and Alternative Sub-Index Weighting Schemes } \\
\hline & & Baseline & Alt 1 & Alt 2 & Alt 3 & Alt 4 & Alt 5 & Alt 6 & Alt 7 & Alt 8 & Alt 9 \\
\hline \multirow{2}{*}{$\begin{array}{l}\text { Vulnerability } \\
\text { Sub-Indices }\end{array}$} & Environmental & $40 \%$ & $40 \%$ & $40 \%$ & $40 \%$ & $50 \%$ & $50 \%$ & $50 \%$ & $60 \%$ & $60 \%$ & $60 \%$ \\
\hline & Socio-Economic & $60 \%$ & $60 \%$ & $60 \%$ & $60 \%$ & $50 \%$ & $50 \%$ & $50 \%$ & $40 \%$ & $40 \%$ & $40 \%$ \\
\hline \multirow{3}{*}{$\begin{array}{l}\text { Resilience } \\
\text { Sub-Indices }\end{array}$} & Individual/Household & $33 \%$ & $30 \%$ & $40 \%$ & $30 \%$ & $40 \%$ & $30 \%$ & $30 \%$ & $40 \%$ & $30 \%$ & $30 \%$ \\
\hline & Neightborhood & $33 \%$ & $40 \%$ & $30 \%$ & $30 \%$ & $30 \%$ & $40 \%$ & $30 \%$ & $30 \%$ & $40 \%$ & $30 \%$ \\
\hline & Government/Institution & $33 \%$ & $30 \%$ & $30 \%$ & $40 \%$ & $30 \%$ & $30 \%$ & $40 \%$ & $30 \%$ & $30 \%$ & $40 \%$ \\
\hline
\end{tabular}

During category recalculation, any changes to the high-risk categories were recorded, with attention paid to neighborhoods which remain in these high-risk categories across weighting scenarios. Table 19 below depicts changes to the high-risk categories due to alternative weighting schemes, indicating the neighborhoods which moved into or dropped out of these categories. 
Table 19. Changes to High-Risk Neighborhood Categorizations Due to Alternative Weighting Schemes

\begin{tabular}{|c|c|c|c|c|c|c|c|c|c|c|}
\hline \multirow{2}{*}{\multicolumn{2}{|c|}{$\begin{array}{c}\text { Type of Categorization } \\
\text { Change }\end{array}$}} & \multicolumn{9}{|c|}{ Alternative Weighting Schemes } \\
\hline & & Alt 1 & Alt 2 & Alt 3 & Alt 4 & Alt 5 & Alt 6 & Alt 7 & Alt 8 & Alt 9 \\
\hline$\downarrow$ & $\begin{array}{l}\text { Dropped From } \\
\text { 'Very High' to 'High' }\end{array}$ & - & - & - & 61 & 61 & 61,62 & 61,71 & 61 & $61,62,71$ \\
\hline$\downarrow$ & $\begin{array}{l}\text { Dropped From } \\
\text { 'High' to 'Medium' } \\
\text { or Lower }\end{array}$ & - & 74 & - & 74,55 & 55 & - & $\begin{array}{c}74,55,72, \\
60\end{array}$ & $\begin{array}{c}74,55,72, \\
60\end{array}$ & $\begin{array}{c}74,55,72, \\
60,59\end{array}$ \\
\hline$\uparrow$ & $\begin{array}{l}\text { Raised From } \\
\text { 'Medium' to 'High' }\end{array}$ & - & 33 & - & 33 & 33 & 33 & - & 33 & 33 \\
\hline
\end{tabular}

The categorizations of four neighborhoods changed consistently (more than half of the time) across weighting schemes. These particular neighborhoods have vulnerability and resilience scores which are very near the chosen information class boundaries (standard deviation breaks), and therefore their risk categorizations are highly sensitive to the choice of weighting scheme. ${ }^{24}$ Choosing alternative weighting schemes Alt 4 through Alt 9 results in neighborhood \#61 (Carr Square) dropping from the 'Very High' to 'High' risk classification. Those same schemes also result in neighborhoods \#74 (Baden) and \#55 (Kingsway East) dropping from the 'High' to 'Medium' risk classification. Importantly, more than half of the alternative weighting schemes result in neighborhood \#33 (Peabody-Darst-Webbe) raising from the 'Medium' to the 'High' risk classification.

Although the low instances of neighborhoods changing classifications due to alternative weighting suggests an acceptably low amount of classification uncertainty in the model, it is still necessary to decide how to represent the few neighborhoods

\footnotetext{
${ }^{24}$ When an object to be classified falls close to, or directly on top of, a discrete information class boundary, the object can be said to have a highly uncertain classification. This uncertainty may be addressed using a fuzzy classification system with non-discrete information class boundaries (Zadeh 1976).
} 
identified as having greater sensitivity to weighting schemes. If left unchanged, the relative resilience/risk categorizations (as rendered in Figure 18), which use the baseline weighting scheme, would over-represent the risk in Baden, Carr Square and Kingsway East; it would also mask the elevated risk in Peabody-Darst-Webbe. A map which uses banded colors to portray the uncertainty of classification in these neighborhoods may suffice to communicate this important information to decision makers (see Figure 20).

The most important mitigation information for St. Louis emergency managers to be gleaned from the above sensitivity analysis is the list of neighborhoods which remain within the same high-risk classifications despite variations in sub-index weighting. These are the neighborhoods which can be described with greater certainty as being threatened with particularly high levels of various hazards while possessing particularly low levels of resilient characteristics. For example, no matter what weighting scheme was applied, neighborhood \#2 (Patch) and \#75 (Riverview) were classified as 'Very High'; likewise, only two alternate weighting scheme choices challenged the 'Very High' risk classification for neighborhoods \#71 (Mark Twain) and \#62 (Columbus Square). Seven out of nine neighborhoods originally classified as 'High' risk were similarly classified in that category across alternative weighting schemes: \#19 (Gravois Park), \#17 (Mount Pleasant), \#72 (Walnut Park East), \#18 (Marine Villa), \#60 (St. Louis Place), \#37 (Midtown) and \#64 (Near North Riverfront). Although this sensitivity analysis can help highlight the neighborhoods which should be given high priority in disaster mitigation strategies, information about which resilience characteristics should 
Figure 20. Example Fuzzy Representation of Neighborhoods With Uncertain Relative Resilience Classifications

\section{Relative Resilience of St. Louis Neighborhoods}

1. Carondele

3. Holy Hills

4 Boulevard Heights

6. Princeton Heigts

7. South Hampton

9 Lindernood Pat

10. Elendale

11. The Hill

13. Southwest Garden
14. Noren Hameton

15. Tower Grove South

16. Dutchtown

17. Mount Pleasart

18. Marne Vila

19. Gravois Par

20. Kosouskio

21. Sousd Perton Part

23. Mckonley Heights

24. Fox Park

25. Tower Grove East

27. Shan

28. Mcriee Tow

29. Tittany

31. Berton Park Weet

32 Latoryete Square

33. Pestody Darst Wetce

34. LaSalle Park

35. Dowrtown

36. Dowrtown West

37. Mation

38. Fonses Pad Sath Ex

9. Forest Paks Sauth Ea
40. Kings Oak

41. Chetrenam

42 Cianon Trm

43. Franz Park

4 Hipointe

45. Wydown Suniver

45. Shinker Debaliviere

47. Debainere Place

49 Vistation Park

50. Wells Goodiellow

51. Acadeny

52. Kangoway Wed

53. Fourtain Pak

54. Lewis Place

55. Kimgsway East

57. The Vile

57. The Vile

59. Jeft Vandertiou

60. Se Laus Place

61. Carr Square

62 Coluntus Square

63. Old Noen ST Louis

4. Near Noten Fivetron

65. Hyd Park

67. Fainground Neigrbohood

68. OFalion

70. Mark Twain 170 induatrial

1. Mark Twain

72. Walnut Pank East

73. Norm Pointe

75. Riverver

76. Warnut Pank Weet:

77 Covenant Bu Grand Certe

79. Norhition Riverigronts

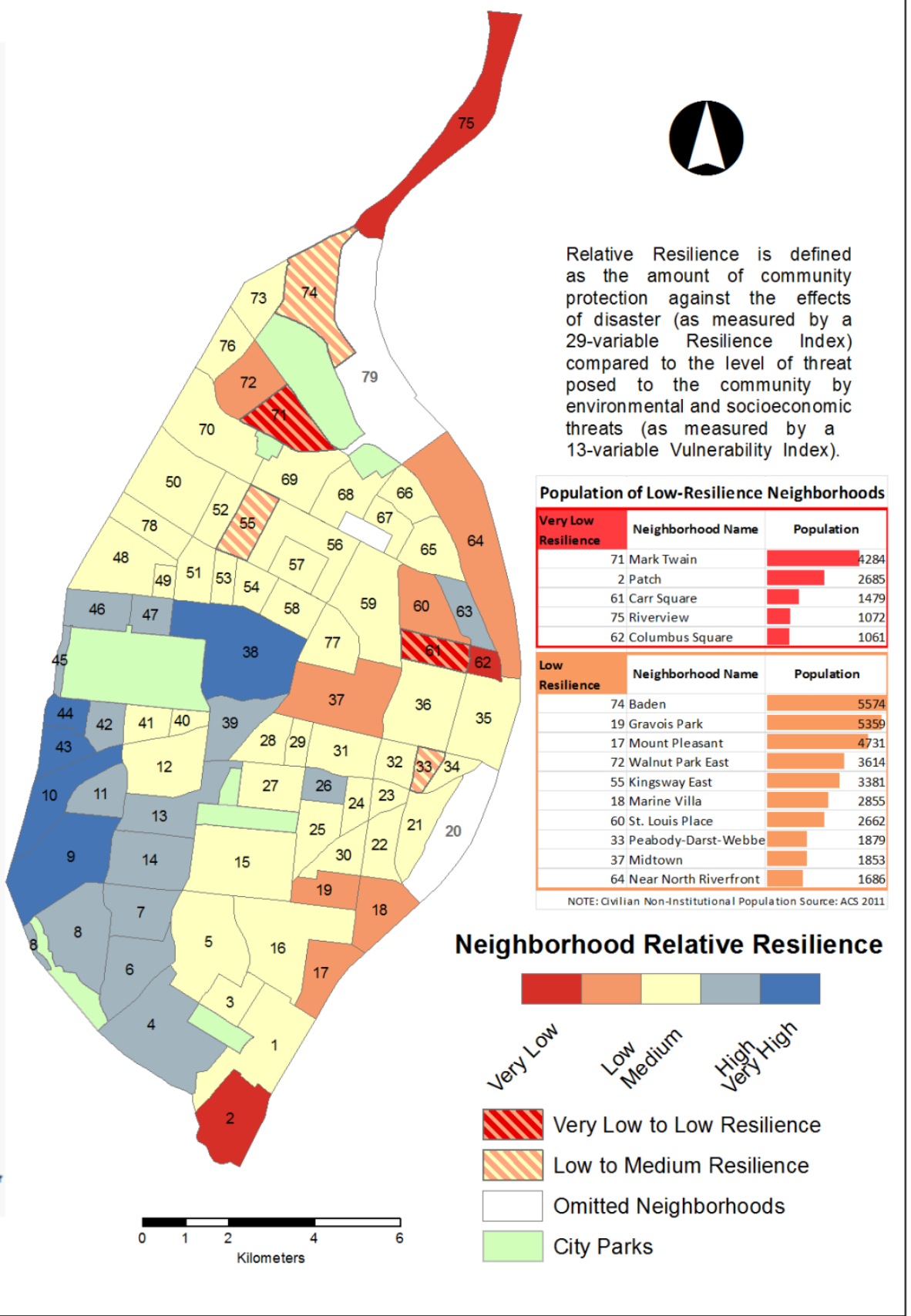


be targeted with such strategies is derived separately in analysis of DEMATEL results (see Section 6.4).

While the internal validity of the proposed community resilience model is judged to be acceptable in terms of the above sensitivity analysis of sub-index weighting, the effect of individual variable weighting also contributes significantly to model uncertainty (Tate 2012). Though beyond the scope of this study, important information about the effects of alternative variable weighting may be uncovered through a Monte Carlo simulation. However, because additional methodological scrutiny was given to variable weighting through the proposed knowledge-based AHP weighting system, it is argued that model sensitivity has been sufficiently addressed.

\subsection{External Validation: Model Correlation to Observed Disaster Resilience}

The additive indices used in this study were comprised of a large and very broad set of variables meant to represent most of the vulnerable and resilient characteristics of populations in the study area. As such, these indices are expected to capture the general sense of a community's protection against the effects of disaster, which may arise from any number of environmental threats modeled. Indices which attempt to construct a general/comprehensive model of disaster resilience may not neatly explain how a community responds to a particular extreme environmental event. An assumption that all modelled resilience characteristics come into play during all disasters is probably not accurate, because communities may respond in very specific ways, and by limited means, after an extreme event. The error of this assumption is 
compounded by the fact that resilience and vulnerability variables in this study represent processes which operate on a variety of complex and dynamic spatial and temporal scales. However, traditional statistical correlation analysis requires this assumption when using the totality of the index to explain a dataset representing observed resilience, even if that resilience could be better explained by a handful of variables relevant to a specific extreme event. Even if strong statistical correlation is not expected, there is still pertinent information to be gleaned from a spatial comparison of modelled community resilience with datasets which show how communities interact in the face of different types of extreme events.

\subsubsection{Case \#1: Extreme Heat-Related Fatalities}

One study-area wide dataset representing St. Louis neighborhood interaction with a slow-onset environmental hazard is a geocoded set of addresses where 18 heatrelated fatalities occurred within St. Louis City during an extreme heat wave and accompanying intense drought in summer 2012 (see Figure 21). Heat-related deaths were dispersed throughout the City, with only two (12\%) occurring within neighborhoods categorized as having 'High' or 'Very High' relative resilience. Sixteen (88\%) of heat-related deaths occurred in neighborhoods categorized as having 'Medium', 'Low' or 'Very Low' relative resilience. This suggests that the resilience model utilized in this thesis has captured qualities and processes in high-resilience neighborhoods which protect vulnerable populations from extreme heat. However, heat deaths did not all cluster within neighborhoods identified as having the lowest 
Figure 21. Resilience Model Validation With Heat Death Data

\section{Correlation of Summer 2012 Heat Deaths With Neighborhood Relative Resilience Categories}
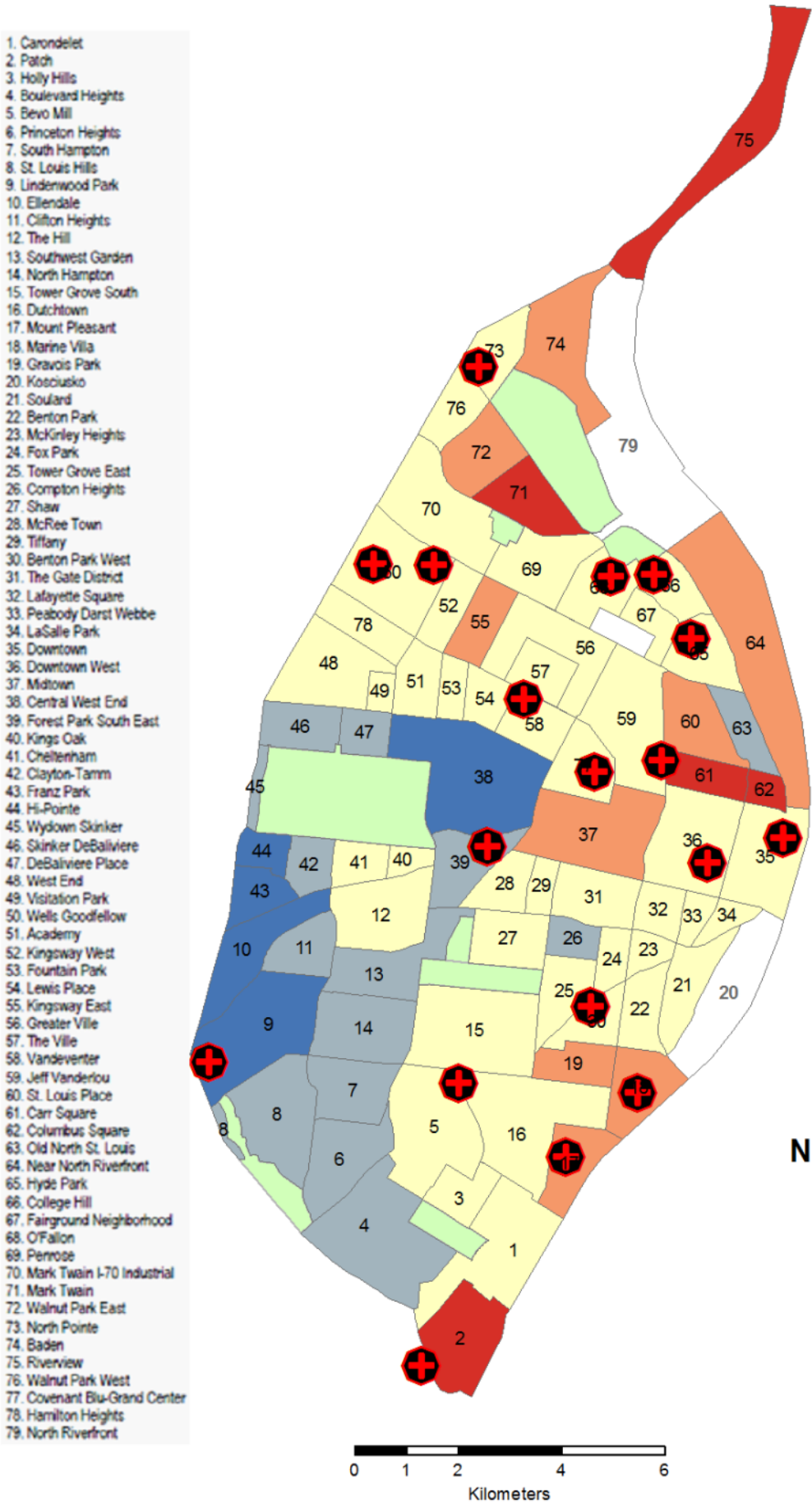

\begin{tabular}{|l|c|c|}
\hline $\begin{array}{c}\text { Resilience } \\
\text { Category }\end{array}$ & $\begin{array}{c}\text { Total \# of } \\
\text { Deaths }\end{array}$ & Percent \\
\hline Very Low & 1 & $6 \%$ \\
\hline Low & 2 & $11 \%$ \\
\hline Medium & 13 & $72 \%$ \\
\hline High & 1 & $6 \%$ \\
\hline Very High & 1 & $6 \%$ \\
\hline
\end{tabular}

Geographically-Weighted Regression Results

INPUT PARAMETERS:

Dependent Variable - Heat Deaths Independent Variable - Neighborhood relative resilience scores transformed to continuous variable (vulnerability score minus absolute resilience score) Weights - Neighborhoods 2011 population

Kernel - Fixed bandwidth @ 6178m OUPUT RESULTS

Correlation Coefficient: .0952

\section{Neighborhood Relative Resilience}

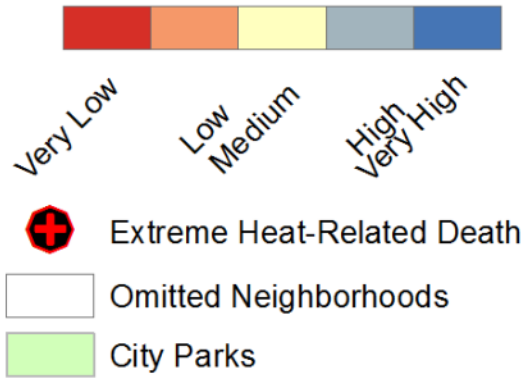


categories of resilience - the vast majority occurred in 'Medium' resilience neighborhoods. This demonstrates that locations of deaths from extreme heat waves may involve more factors than simply highest overall risk. The fact that all of the deceased were over the age of 50 suggests that specific factors such as age and health may have also accounted for the location of the death.

\subsubsection{Case \#2: Population Loss Due to Economic Shocks}

An important part of the definition of disaster used in this study as it relates to human social systems involves how an extreme environmental may overwhelm a population's coping mechanisms, push a social system into an unfavorable state, and lead to some peoples' decision to move elsewhere. While severe, fast-onset natural disasters such as a tornado's destruction of a city certainly have the potential to instigate such change, it is also important to consider slower-onset extreme events operating on longer time-scales, such as sudden economic shocks. The global financial crisis of 2008 caused such a shock for consumer finances and household balance sheets, precipitating a national mean household wealth decline of $15 \%$ and a median household wealth decline of 39\% (Federal Reserve Bank of St. Louis, 2013). In St. Louis, the loss of financial security for households due to economic downturn probably contributed to the observed sharp drop in population experienced by the city in the years immediately following the crisis. ${ }^{25}$ The effect of this sudden population decline may have been

\footnotetext{
${ }^{25}$ The nature of the financial crisis' effects on St. Louis has not been studied. However, it is assumed here that many St. Louisans experienced foreclosure and loss of jobs, and that socioeconomic conditions in the City were worsened in general by the attenuating negative effects of the economic downturn.
} 
pronounced since it came on the heels of a hopeful period of post-millennial population growth after more than 30 years of steady decline (see Figure 22). However, it is expected that some neighborhoods within St. Louis weathered the financial crisis better than others, and that the indicators of resilience included in this study may have helped insulate these neighborhoods from the socioeconomic stressors instigating sharp population decline.

Figure 22. Abrupt Population Loss in St. Louis Following 2008 Financial Crisis

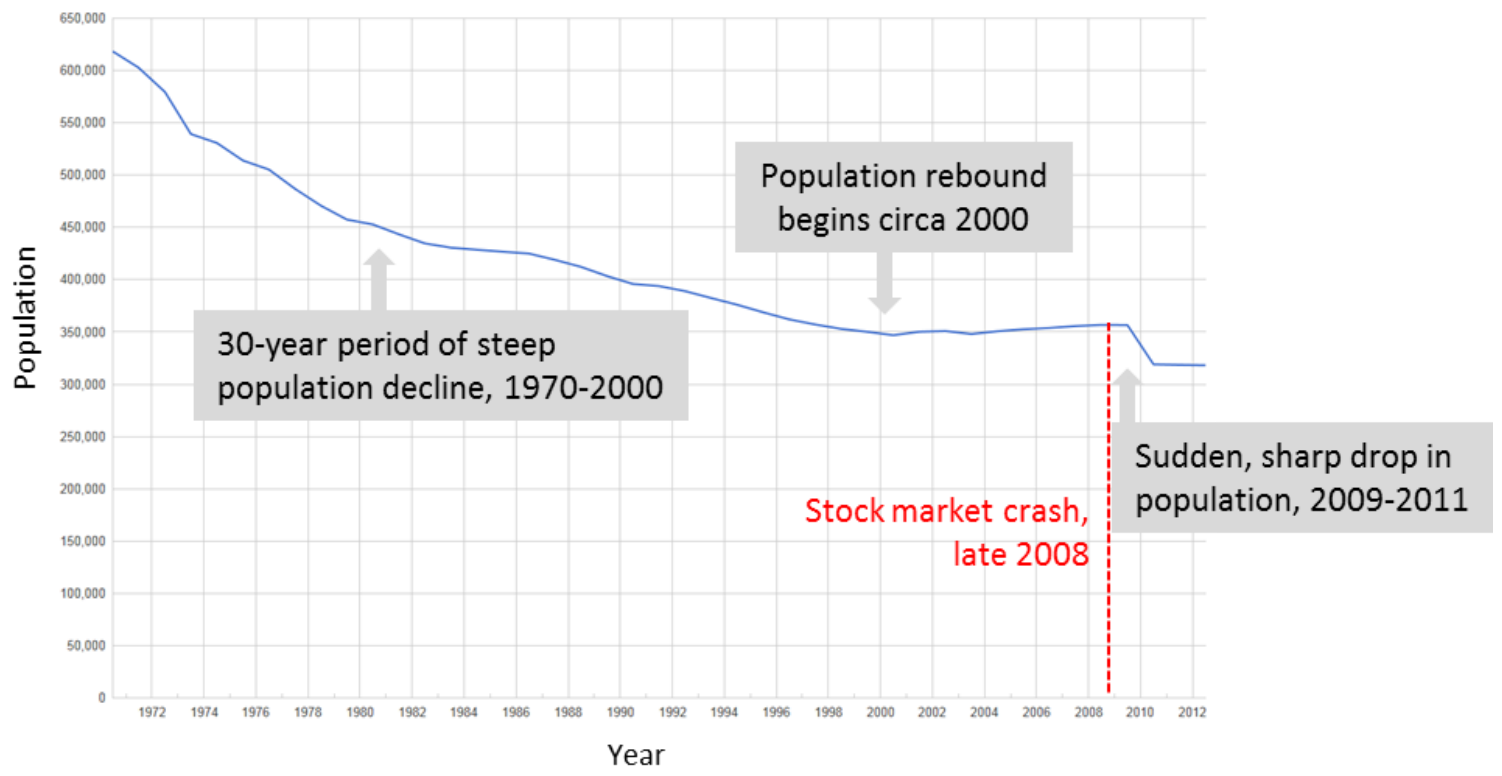

To test this hypothesis, geographically weighted regression was conducted to evaluate how well St. Louis neighborhood population change - as calculated from ACS 2011 and ACS 2012 5-year population estimates - can be explained by neighborhood relative resilience scores. The spatial variable for geographic weighting was a fixed 
Figure 23. Resilience Model Validation With Post-Economic Shock Population Change

\section{Correlation of 2006-2012 Population Change With Neighborhood Relative Resilience Scores}

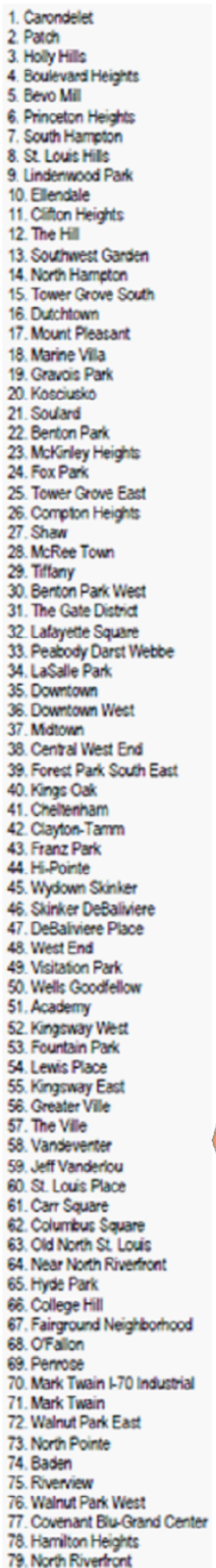

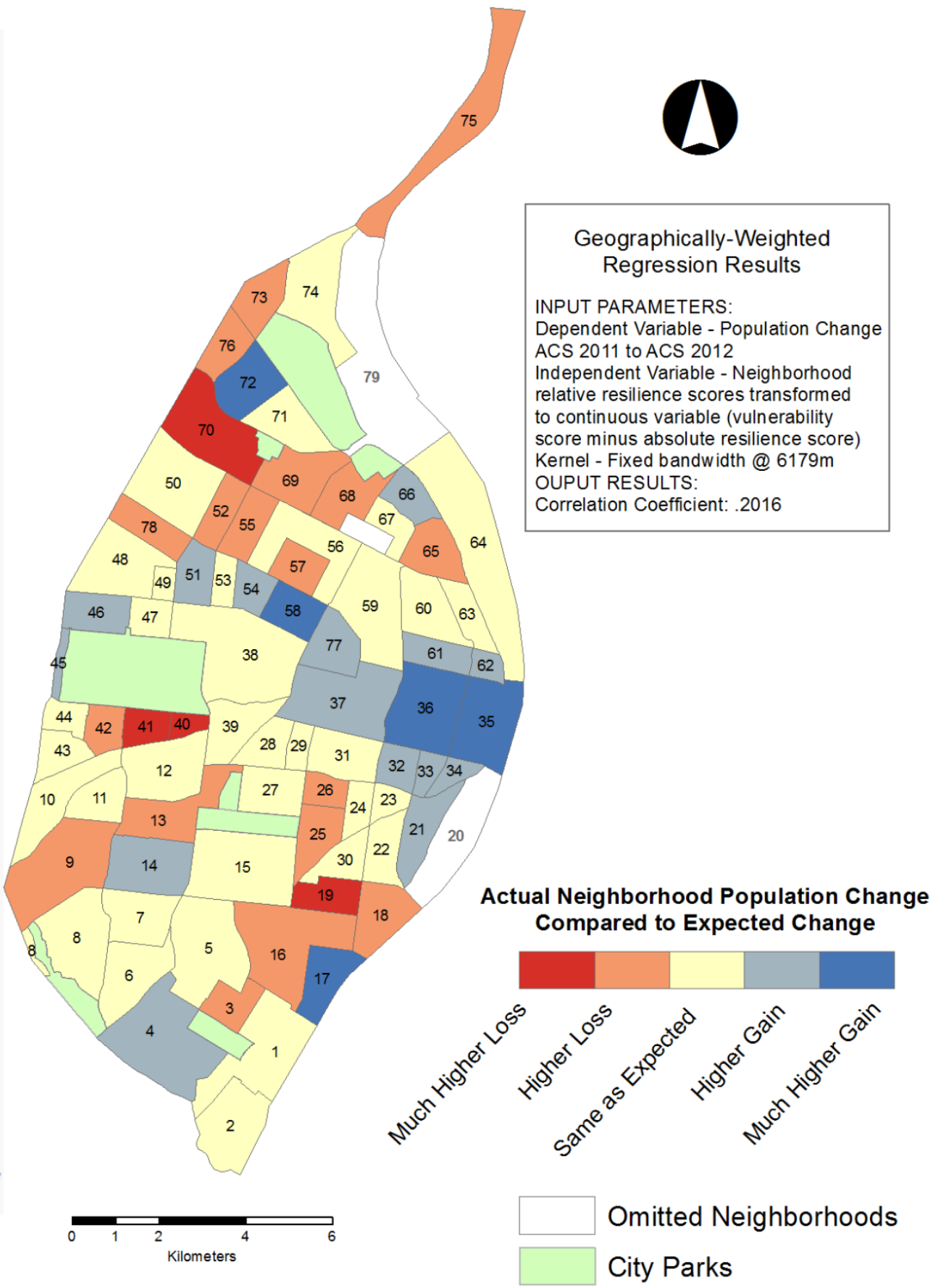


kernel at 6279 meters. In order to use a continuous variable for relative resilience instead of categories, it was decided to use the difference between each neighborhood's raw vulnerability and absolute resilience index score (Vulnerability Score - Resilience Score $=$ Relative Resilience Score). Both the independent and dependent variables are somewhat imprecise for the phenomenon under study. For example, the ACS 2011 figure is a single estimate for years 2006-2010 while ACS 2012 estimates for years 2007-2011; therefore the dependent variable includes some population change which occurred before the financial crisis. Also, as discussed earlier in the rationale for using a semantic, category-based approach for vulnerability and absolute resilience index comparison, using raw scores for comparison/quantification is unwise since the separate indices are not in the same scale. Nonetheless, results of regression (shown in Figure 23) indicate a significant correlation, with an $r^{2}$ coefficient of .2016. Although the amount of variation explained by the regression is very low (and expected, since it is assumed population change and relative resilience have a nonlinear relationship), there is interesting spatial variability in the amount of observed versus expected population change, with the central business corridor including Downtown, Downtown West and Midtown experiencing much higher growth vis-à-vis their relative resilience scores. This may suggest that the more numerous or higherpaying job opportunities in these central business district areas acted as a strong buffer to population decline. Higher-than expected population loss clusters in areas of North and South City, with most neighborhoods identified as having low relative resilience also experiencing higher-than-predicited population decline. This may suggest that negative 
socioeconomic feedbacks associated with the economic downturn were intensified in areas lacking the indicators of resilience in the chosen model.

\subsubsection{Case \#3. Resident Requests for Government Assistance after Windstorms}

Some of the clearest evidence for a lack of (or an overwhelming of) householdand community-level disaster resilience can be derived from data on household requests for government financial and/or labor assistance following an extreme event. A notable recent example of this occurred during the winter and early spring of 2011, when a series of unseasonably powerful windstorms affected large portions of north St. Louis, including an EF-0 strength tornado which occurred on New Year's Eve 2010 (St. Louis Post-Dispatch 2010). Between January and March 2011, 175 households made telephone requests to St. Louis City Emergency Management Agency for various disaster recovery-related services, including financial assistance for the repair or replacement of damaged property, and for labor assistance to clean up personal property or repair roof damage (STL Citizens Service Bureau 2013). According to St. Louis City records, most of these callers cited lack of insurance as the reason for the request, though some cited health-related issues which prevented them from making repairs themselves, or lack of family contacts willing to help (STL Citizen Service Bureau 2013).

Even though the act of requesting disaster assistance from a municipal government can be viewed as a type of resilient action (since it takes advantage of government-level response/recovery resources), it suggests that sources of household and community level resilience have been overwhelmed. Also, in this case, there was 
Figure 24. Resilience Model Validation With Storm-Related Requests For Assistance

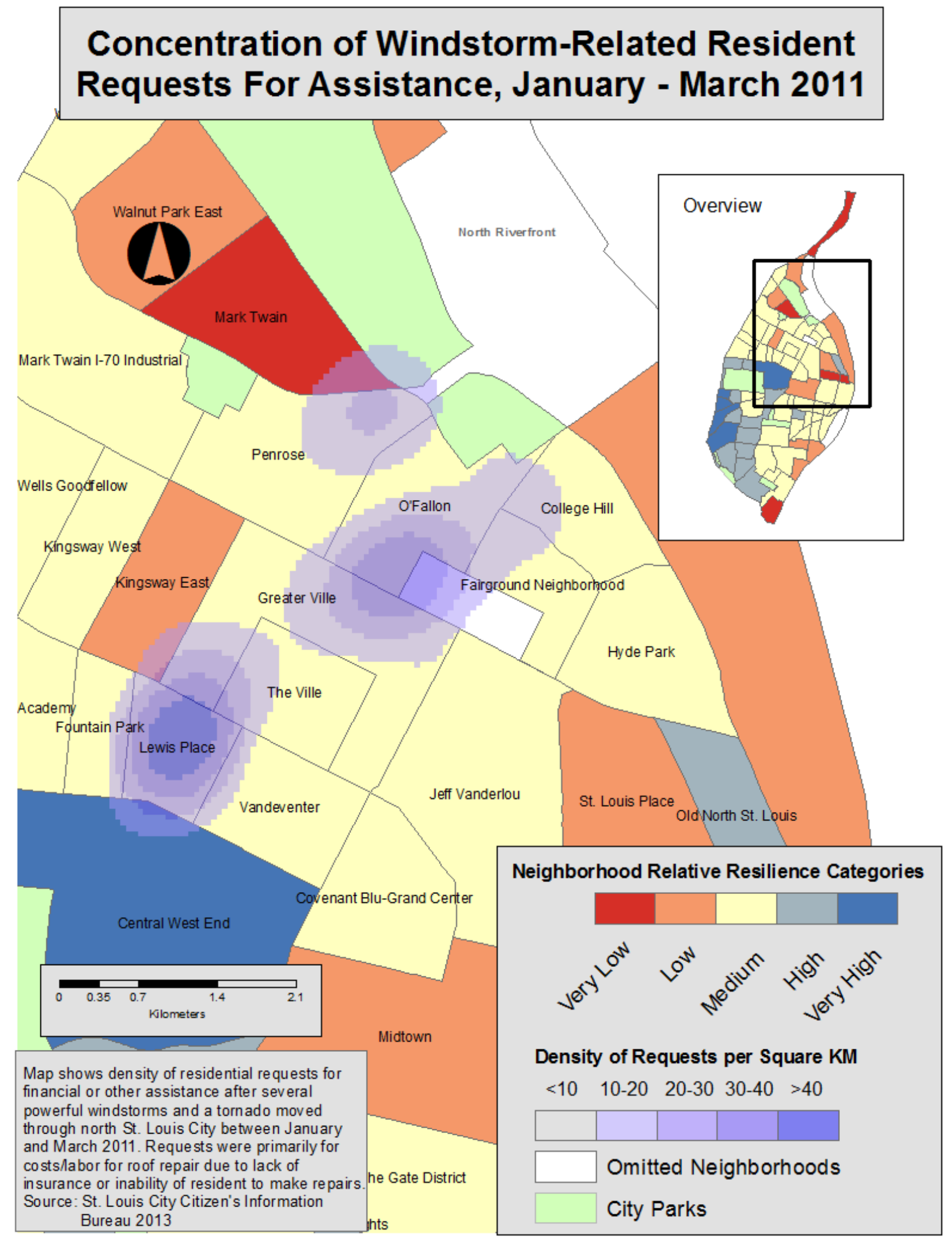


no program of household disaster assistance, financial or otherwise, offered by the City of St. Louis; the requests were eventually routed from the City Emergency Management Agency office to a local non-profit disaster relief agency, Americorps St. Louis, which provided free roof tarping and cleanup services.

Because the 2011 windstorms affected relatively few neighborhoods in North St. Louis, geographically weighted regression was not performed to correlate neighborhood relative resilience scores with number of requests for assistance per neighborhood. However, a point density layer representing concentration of requests for assistance per square kilometer was created, as shown in Figure 24 above. The density surface was classified in 5 colors representing increases of 10 requests per square kilometer. As in the occurrences of heat deaths shown in Figure 21, the important information for emergency managers rendered in Figure 24 of disaster assistance requests is that, while households in high-resilience neighborhoods (such as Central West End) are not likely to request government assistance, the requests which do occur may not simply cluster in neighborhoods identified as having the lowest resilience.

\subsection{Model Correction for Validation Results}

In fact, the external validation conducted here with three case study datasets demonstrates that the original choice of categorization of neighborhood resilience may have misrepresented the elevated risk in some of the neighborhoods categorized as having 'Medium' relative resilience. This can be remedied simply by reassigning relative resilience categories so that less neighborhoods fall under the 'Medium' category (see 
Figure 25). Only neighborhoods on the diagonal retain a 'Medium' (yellow)

classification, while neighborhoods in the row above are reclassified as 'Low' (orange) and those below are reclassified 'High' (light blue).

Figure 25. Reclassification of Original Relative Resilience Categories Original Classification: 44 Neighborhoods Classified as 'Medium'

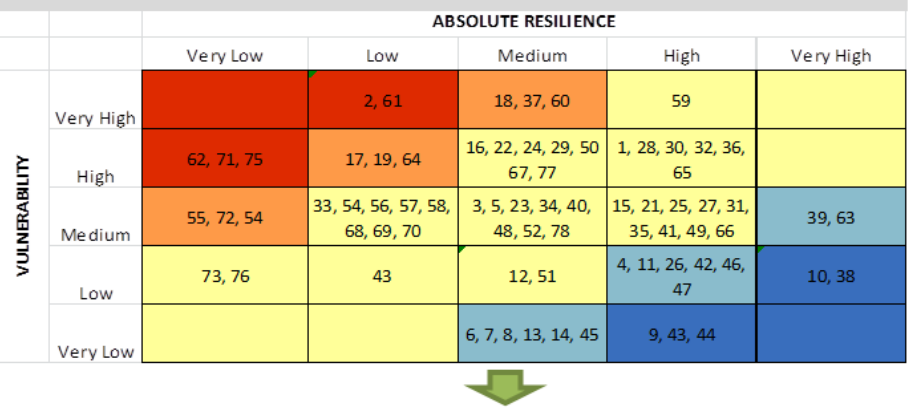

New Classification: 15 Neighborhoods Classified as 'Medium'

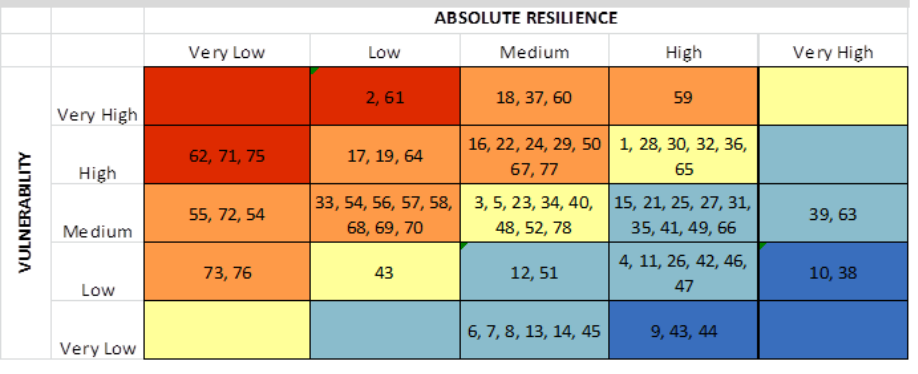


Figure 26. Reclassified Relative Resilience Categories with Fewer 'Medium' Neighborhood Categorizations

\section{Relative Resilience of St. Louis Neighborhoods}
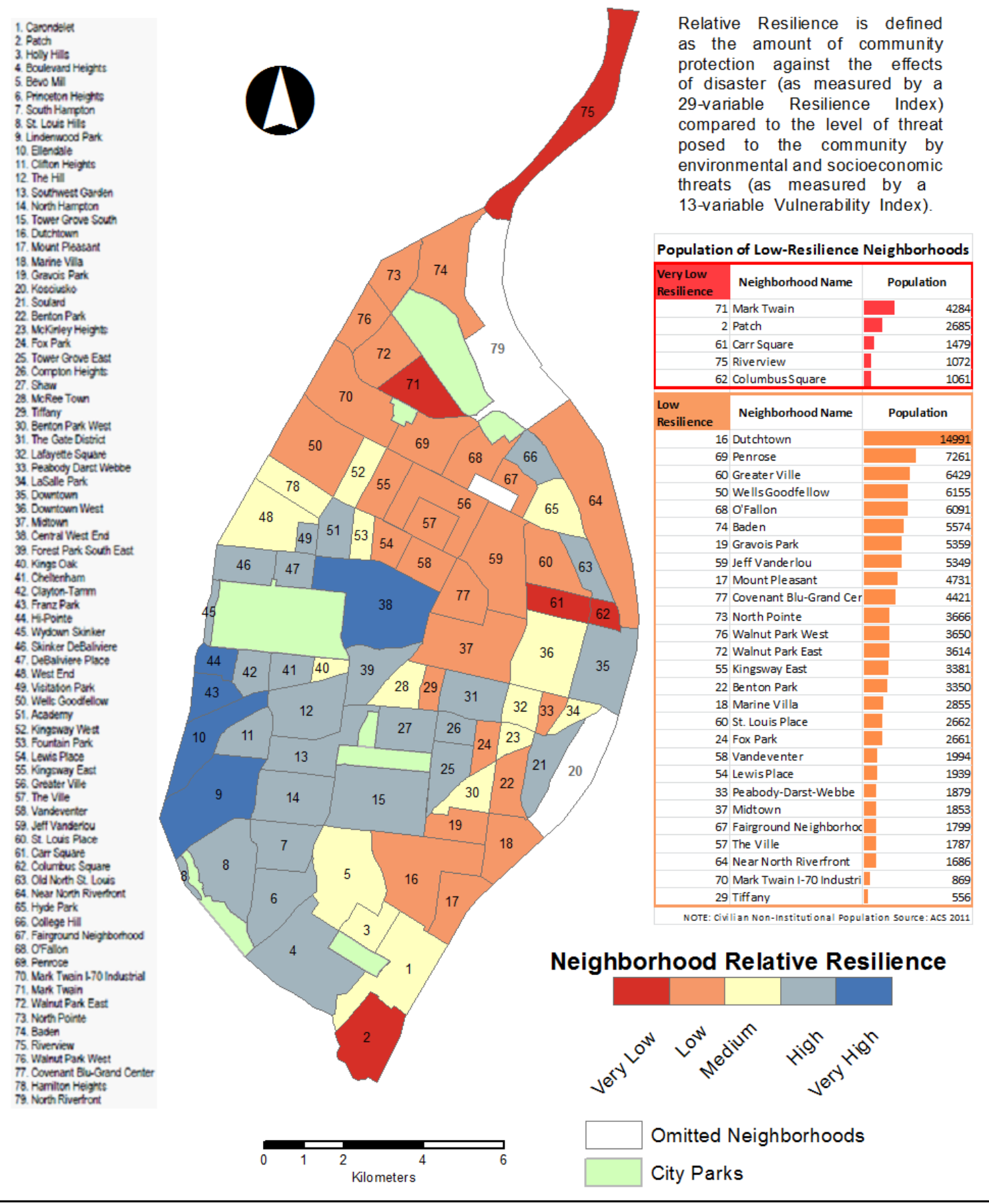
When these new categories are visualized (see Figure 26 above), better agreement with the validation datasets can be observed. Also, the reclassification requires neighborhoods in the new 'Medium' category to have matching vulnerability and absolute resilience classifications, which is in line with the assumption that a certain level of resilience may 'cancel out' the same level of vulnerability. However, the recategorized relative resilience classification trades better information about 'Medium' resilience neighborhoods for a substantially larger set of 'Low' resilience neighborhoods for emergency managers to more closely monitor. In fact, the original categorization of the lower resilience neighborhoods encompassed a population of 44,175 in 14 neighborhoods, equivalent to $14 \%$ of the total St. Louis population - a reasonable amount of people for whom to plan mitigation strategies and emergency response policies. The new categorization, on the other hand, encompasses 117,143 people in 32 neighborhoods, equivalent to $37 \%$ of the total city population. Nonetheless, it is probably better to choose a representation which correlates better with observed levels of disaster resilience, as represented by the validation datasets presented.

\subsection{Spatial Patterns of High and Low Resilience in St. Louis}

Whatever the choice of parameterization and representation decided in the validation stage, there is clear spatial clustering of high and low relative resilience in St. Louis neighborhoods. This was expected, since many of the indicators used in this study had similarly clustered values (see Appendices A and B for maps of all individual indicator variables in the vulnerability and absolute resilience indices). The final clustering of high and low resilience neighborhoods also conforms to the narrative of 
public health officials and resident focus groups reported in St. Louis City Department of Health's publication Understanding Our Needs (STL Health Dept. 2012). This publication describes North St. Louis as an economically depressed area suffering from high crime and the effects of outmigration, as opposed to an affluent west and southwest section of the city with wealthier households and less socioeconomic stressors.

Statistically significant groupings of neighborhoods with the same relative resilience category can be identified by using the Local Moran's I statistic, which

Figure 27. Significant Clustering of High and Low Relative Resilience Neighborhoods
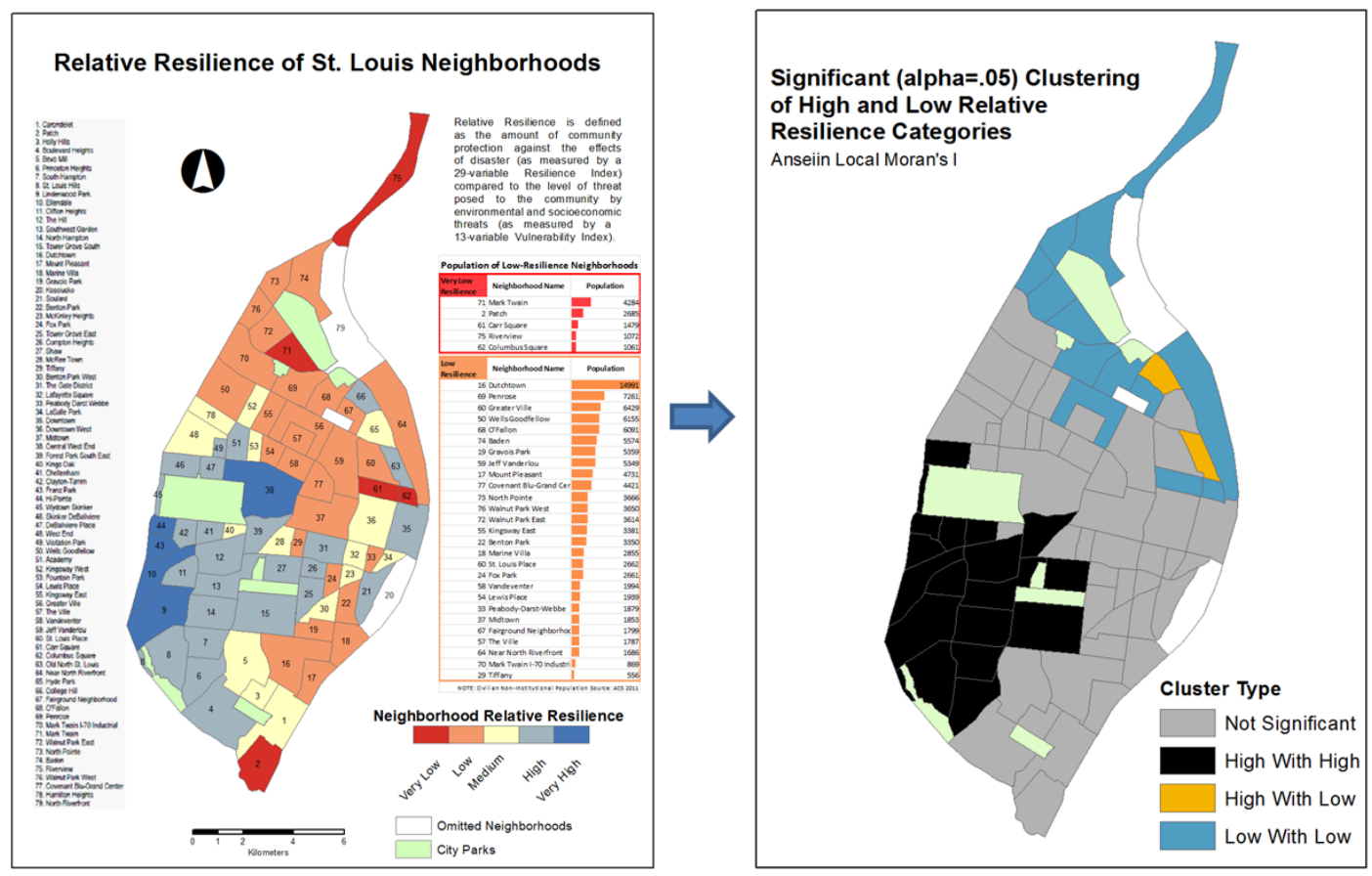

assesses categories of surrounding neighborhoods and their defined weights (in this case, neighborhoods were weighted using their 2011 population) to evaluate clustering of high and low resilience neighborhoods. This statistic also identifies neighborhoods surrounded with significantly different categories of resilience. Results (see Figure 27) indicate that much of the Southwest portion of the city had significant instances of high- 
resilience neighborhoods being surrounded by other high-resilience neighborhoods.

The opposite trend can be observed in the northern part of the city, with significant instances of low-resilience neighborhoods surrounded by other low resilience neighborhoods. Interestingly, there are two neighborhoods in North St. Louis identified as having particularly high levels of resilience compared to their neighbors. This finding may suggest the existence of a combination of important resilience-boosting processes unique to these neighborhoods, and/or points to these neighborhoods being less affected by negative influences from surrounding areas. The notion that the levels of resilience in a particular neighborhood may influence those of surrounding neighborhoods will be discussed in the next section.

\subsection{Autocorrelation and Spatial Influence of Neighborhood Resilience}

So far this chapter has mostly discussed the results of the relative resilience calculation in terms of each neighborhood's discrete resilience classification, not in terms of how the spatial characteristics of each neighborhood might influence the level of resilience in others. Likewise, the apportionment of variable data and calculation of raw neighborhood scores for vulnerability and absolute resilience, as well as the final categorization of relative resilience, operated on the assumption that each neighborhood's combined attributes do not exert any influence on their neighbors.

However, as components of complex, multi-scale social systems, neighborhoods can be assumed both to exert influence on and receive influence from surrounding 
neighborhoods. ${ }^{26}$ These influence/feedback relations would be very difficult to model based on a single, 'snapshot' studies of resilience using temporally-limited data; as in the modelling of physical systems, it would be better to study the mechanics of how socioeconomic relations change through time, in order to arrive at an empirically-based model of how these feedback relations 'work' in the real world. However, even without longitudinal data, observed spatial characteristics of neighborhoods, as measured by various spatial clustering and geographic weighting statistics, can be used to further modify the relative resilience model.

Spatial statistics, many of which were developed in line with Tobler's Law that 'things near each other are more alike than things far apart', might be used to model how the spatial attributes of communities influence their neighbors. For example, it may be correct to assume that a positive feedback relationship exists between adjacent neighborhoods with similarly high levels of resilience, such that both neighborhoods' resilience is mutually sustained or even magnified by this connection. An opposite, negative feedback relationship may exist between adjacent neighborhoods of low levels of resilience. The strength of this feedback may depend on a number of factors, such as the neighborhoods' population, the distance between neighborhood centroids, the amount of time each has had strong positive or negative resilience attributes, or the size of the cluster of neighborhoods with similar levels of resilience, etc. Factors such as neighborhood relationships and contiguity can be modeled as a spatial weights matrix,

\footnotetext{
${ }^{26}$ These influence relationship, of course, are not limited to immediately bordering neighborhoods; they should be assumed to radiate out with diminishing strength according to distance and the types of dependencies and feedback relations on much broader spatial scales.
} 
which is similar to a digraph in that it estimates the strength of the spatial relationship between neighborhood $i$ and all other neighborhoods $j$ that are within a specified spatial relationship with each other, (i.e. based on centroid distance or edge contiguity, etc.). A spatial weights matrix can be easily rendered as a 3-column table showing the quantified distance- and/or contiguity-related weight between an 'origin' neighborhood and its 'destination' neighborhoods, with the weights quantified as the inverse of the number of neighbors(see Table 20 for part of the spatial weights matrix computed for St. Louis neighborhoods, which shows weights for contiguous neighbors for neighborhoods \#1-5).

Table 20. Spatial Weights Matrix Computed for Neighborhoods \#1-5

\begin{tabular}{|r|r|r|}
\hline $\begin{array}{c}\text { ORIGIN } \\
\text { Neighborhood \# }\end{array}$ & $\begin{array}{c}\text { DESTINATION } \\
\text { Neighborhood \# }\end{array}$ & \multicolumn{1}{l|}{ WEIGHT } \\
\hline 1 & 2 & 0.20 \\
\hline 1 & 3 & 0.20 \\
\hline 1 & 4 & 0.20 \\
\hline 1 & 16 & 0.20 \\
\hline 1 & 17 & 0.20 \\
\hline 2 & 1 & 1.00 \\
\hline 3 & 1 & 0.25 \\
\hline 3 & 4 & 0.25 \\
\hline 3 & 5 & 0.25 \\
\hline 3 & 16 & 0.25 \\
\hline 4 & 1 & 0.25 \\
\hline 4 & 3 & 0.25 \\
\hline 4 & 5 & 0.25 \\
\hline 4 & 6 & 0.25 \\
\hline 5 & 3 & 0.17 \\
\hline 5 & 4 & 0.17 \\
\hline 5 & 7 & 0.17 \\
\hline 5 & 6 & 0.17 \\
\hline 5 & 15 & 0.17 \\
\hline 5 & 16 & 0.17 \\
\hline & & \\
\hline
\end{tabular}

NOTE: Only contiguous neighborhoods were considered in this weights matrix (For example, \#2 Patch has only one contiguous neighborhood, \#1 Carondelet) 
Suppose the spatial weights matrix shows 'origin-destination weights' $W_{1}$ based solely on spatial (distance and contiguity) characteristics, but that these might be further modified to reflect the relative magnitude of origin-destination neighborhood influence based on the population of those neighborhoods. ${ }^{27} \mathrm{~A}$ 'population weight' field $W_{\mathrm{p}}$ was already calculated for neighborhoods for use in the Local Moran's I analysis above (Figure 27), by dividing neighborhood population by the total St. Louis population. The population weights $W_{\mathrm{p}}$ for origin neighborhoods $N_{\mathrm{i}}$ can be appended to the spatial weights matrix. The inverse of $W_{1}$ can then be multiplied by the inverse of the newly appended population weights $W_{\mathrm{p}}$. This results in a new 'distance/population weight' $W_{2}$ for all $N_{\mathrm{i}} \rightarrow N_{\mathrm{j}}$ which has been scaled based on the Origin neighborhood's population. Normalizing $W_{2}$ by dividing each value by the highest weight results in a 'normalized distance/population' weight $W_{\mathrm{n}}$.

\footnotetext{
${ }^{27}$ The following discussion of the new spatial influence measurement method includes notation for each referenced element for the sake of clarity.
} 
Table 21. Normalized Distance/Population Weights $W_{N}$ Calculated for Selected Neighborhoods

\begin{tabular}{|c|c|c|c|c|c|}
\hline $\begin{array}{c}\text { ORIGIN } N_{\mathrm{i}} \\
\text { Neighborhood \# }\end{array}$ & $\begin{array}{l}\text { DESTINATION } \boldsymbol{N}_{\mathrm{j}} \\
\text { Neighborhood \# }\end{array}$ & $\begin{array}{l}\text { O-D Distance } \\
\text { Weight } W_{1}\end{array}$ & $\begin{array}{l}\text { ORIGIN } \\
\text { Neighborhood } \\
\text { Population } \\
\text { Weight } W p \\
\end{array}$ & $\begin{array}{c}\text { O-D Distance / } \\
\text { Population Weight } W_{2}\end{array}$ & $\begin{array}{l}\text { Normalized Distance / } \\
\text { Population Weight } W_{N}\end{array}$ \\
\hline 1 & 2 & 0.20 & 0.0289 & 6.922202604 & 0.0040388 \\
\hline 1 & 3 & 0.20 & 0.0289 & 6.922202604 & 0.0040388 \\
\hline 1 & 4 & 0.20 & 0.0289 & 6.922202604 & 0.0040388 \\
\hline 1 & 16 & 0.20 & 0.0289 & 6.922202604 & 0.0040388 \\
\hline 1 & 17 & 0.20 & 0.0289 & 6.922202604 & 0.0040388 \\
\hline 2 & 1 & 1.00 & 0.0086 & 116.8137803 & 0.0681564 \\
\hline 3 & 1 & 0.25 & 0.0111 & 22.51256101 & 0.0131352 \\
\hline 3 & 4 & 0.25 & 0.0111 & 22.51256101 & 0.0131352 \\
\hline 3 & 5 & 0.25 & 0.0111 & 22.51256101 & 0.0131352 \\
\hline 3 & 16 & 0.25 & 0.0111 & 22.51256101 & 0.0131352 \\
\hline 4 & 1 & 0.25 & 0.0285 & 8.76103352 & 0.0051117 \\
\hline 4 & 3 & 0.25 & 0.0285 & 8.76103352 & 0.0051117 \\
\hline 4 & 5 & 0.25 & 0.0285 & 8.76103352 & 0.0051117 \\
\hline 4 & 6 & 0.25 & 0.0285 & 8.76103352 & 0.0051117 \\
\hline 5 & 3 & 0.17 & 0.0362 & 4.603220031 & 0.0026858 \\
\hline 5 & 4 & 0.17 & 0.0362 & 4.603220031 & 0.0026858 \\
\hline 5 & 7 & 0.17 & 0.0362 & 4.603220031 & 0.0026858 \\
\hline 5 & 6 & 0.17 & 0.0362 & 4.603220031 & 0.0026858 \\
\hline 5 & 15 & 0.17 & 0.0362 & 4.603220031 & 0.0026858 \\
\hline 5 & 16 & 0.17 & 0.0362 & 4.603220031 & 0.0026858 \\
\hline
\end{tabular}

$W_{N}$ represents the strength of the influence that $N_{i}$ exerts on each $N_{\mathrm{j}}$, based on population and spatial characteristics but not the type/magnitude of resilience influence (positive or negative resilience); a value for $\mathrm{N}_{\mathrm{i}}$ 's relative resilience category will provide this, if it shows a positive value for the higher resilience or a negative value for lower ones. Relative resilience category values were already assigned positive/negative values for the purposes of color mapping, with 'Very Low' $=-2.0$, 'Low' $=-1.0$, 'Medium' $^{\prime}=0.0$, 'High'= 1.0 and 'Very High'= 2.0. When these category numbers are appended to the table for each origin neighborhood $N_{\mathrm{i}}$, they can be multiplied by the normalized distance/population weights $W_{\mathrm{N}}$, resulting in a set of directed resilience influences $R_{\mathrm{l}-\mathrm{j}}$ for all $N_{\mathrm{i}} \rightarrow N_{\mathrm{j}}$. 
Table 22. Directed Resilience Influences $R_{\mathrm{l-j}}$ Calculated for Selected Neighborhoods

\begin{tabular}{|c|c|c|c|c|}
\hline $\begin{array}{c}\text { ORIGIN } \boldsymbol{N}_{\mathrm{i}} \\
\text { Neighborhood \# }\end{array}$ & $\begin{array}{l}\text { DESTINATION } \boldsymbol{N}_{\mathrm{j}} \\
\text { Neighborhood \# }\end{array}$ & $\begin{array}{l}\text { Normalized Distance / } \\
\text { Population Weight } W_{\mathrm{N}}\end{array}$ & $\begin{array}{c}\boldsymbol{N}_{\mathrm{i}} \text { Relative } \\
\text { Resilience } \\
\text { Category Number }\end{array}$ & $\begin{array}{c}\text { Directed } \\
\text { Resilience } \\
\text { Influence } \mathbf{R}_{\mathrm{i}-\mathrm{j}}\end{array}$ \\
\hline 1 & 2 & 0.0040388 & 0 & 0 \\
\hline 1 & 3 & 0.0040388 & 0 & 0 \\
\hline 1 & 4 & 0.0040388 & 0 & 0 \\
\hline 1 & 16 & 0.0040388 & 0 & 0 \\
\hline 1 & 17 & 0.0040388 & 0 & 0 \\
\hline 2 & 1 & 0.0681564 & -2 & -0.136312849 \\
\hline 3 & 1 & 0.0131352 & 0 & 0 \\
\hline 3 & 4 & 0.0131352 & 0 & 0 \\
\hline 3 & 5 & 0.0131352 & 0 & 0 \\
\hline 3 & 16 & 0.0131352 & 0 & 0 \\
\hline 4 & 1 & 0.0051117 & 1 & 0.005111732 \\
\hline 4 & 3 & 0.0051117 & 1 & 0.005111732 \\
\hline 4 & 5 & 0.0051117 & 1 & 0.005111732 \\
\hline 4 & 6 & 0.0051117 & 1 & 0.005111732 \\
\hline 5 & 3 & 0.0026858 & 0 & 0 \\
\hline 5 & 4 & 0.0026858 & 0 & 0 \\
\hline 5 & 7 & 0.0026858 & 0 & 0 \\
\hline 5 & 6 & 0.0026858 & 0 & 0 \\
\hline 5 & 15 & 0.0026858 & 0 & 0 \\
\hline 5 & 16 & 0.0026858 & 0 & 0 \\
\hline
\end{tabular}

Summarizing the table by adding all $\mathrm{R}_{\mathrm{i}-\mathrm{j}}$ values for each Destination

neighborhood $\mathrm{N}_{\mathrm{j}}$ results in a total incoming resilience influence $T_{\mathrm{IR}}$ for each

neighborhood, representing the aggregate positive or negative resilience influences

directed at that neighborhood by all other neighborhoods (subject to the imposed

contiguity rule). If a neighborhood has a positive $T_{\mathrm{IR}}$ value, it indicates that more

bordering neighborhoods have high (positive) relative resilience. A positive $T_{\mathbb{R}}$

neighborhood can be said to benefit from a positive resilience feedback from its spatial

relationship with its neighbors, and this effect is magnified if bordering neighborhoods

have higher populations. The opposite is true for a neighborhood with negative $T_{\mathrm{IR}}$

values - It can be said to suffer from a negative resilience feedback given its close spatial 
relationship with low-resilience neighborhoods. Again, this negative feedback can be magnified if surrounding low-resilience neighborhoods have high populations.

If certain neighborhoods have very high or very low $T_{\mathrm{I}}$ scores, it makes sense to change their relative resilience category to reflect this new information. When $T_{\mathrm{IR}}$ values for St. Louis neighborhoods are graphed, the resulting normal distribution, with fewer neighborhoods having very high positive or very low negative $T_{\mathrm{IR}}$ values (see Figure 28), suggests that standard deviational categories may again be appropriately used to set thresholds of $T_{\mathrm{IR}}$ values thus necessitating a relative resilience category change. Using $a+1.5$ and -1.5 standard deviation threshold as a basis for categorization, 8 neighborhoods are identified as having very high negative incoming resilience influence, and 9 neighborhoods are identified as having very high positive incoming resilience influence.

Figure 28. Distribution and Standard Deviation-Based Categories of Incoming Resilience Influence $T_{\mathrm{IR}}$

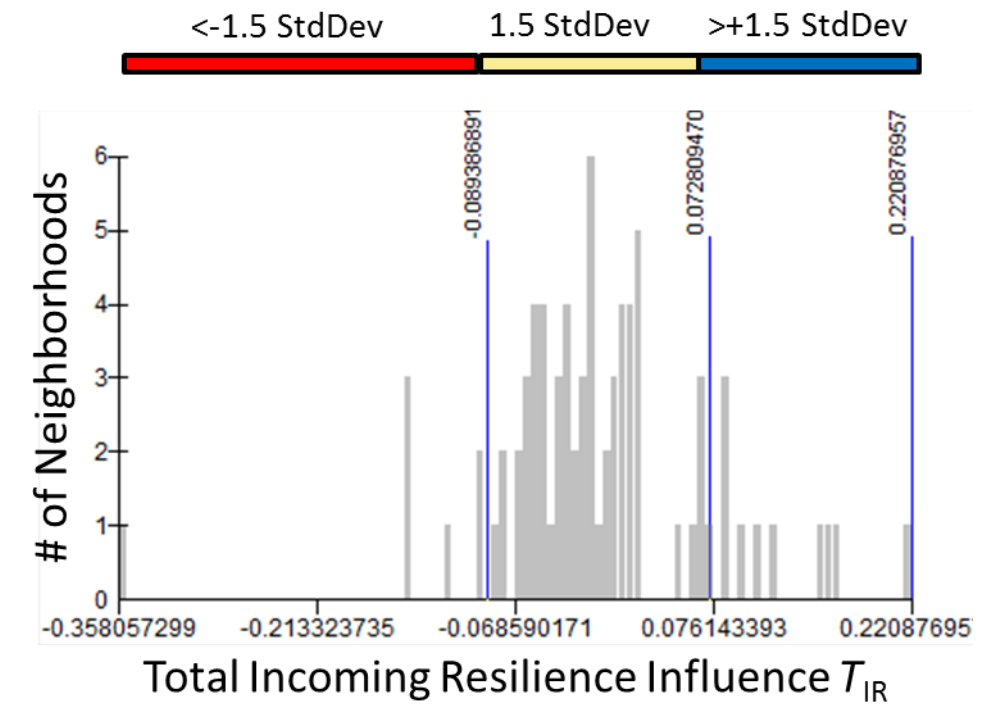


Neighborhoods in the Very High Positive $T_{\mathrm{IR}}$ category are bumped up to the next higher relative resilience category. Neighborhoods in the Very Low Negative $T_{\mathrm{IR}}$ category are bumped down into the next lower relative resilience category. However, the classification of the two neighborhoods identified in the Local Moran's I analysis as having significantly higher relative resilience than their neighbors were not changed, since they are assumed to be somewhat insulated from the influence of surrounding neighborhoods. These changes in neighborhood classification are shown in Figure 29, and the resulting new relative resilience map with neighborhood population tables is shown in Figure 30.

Figure 29. Changes to Relative Resilience Categories after Spatial Influence Analysis

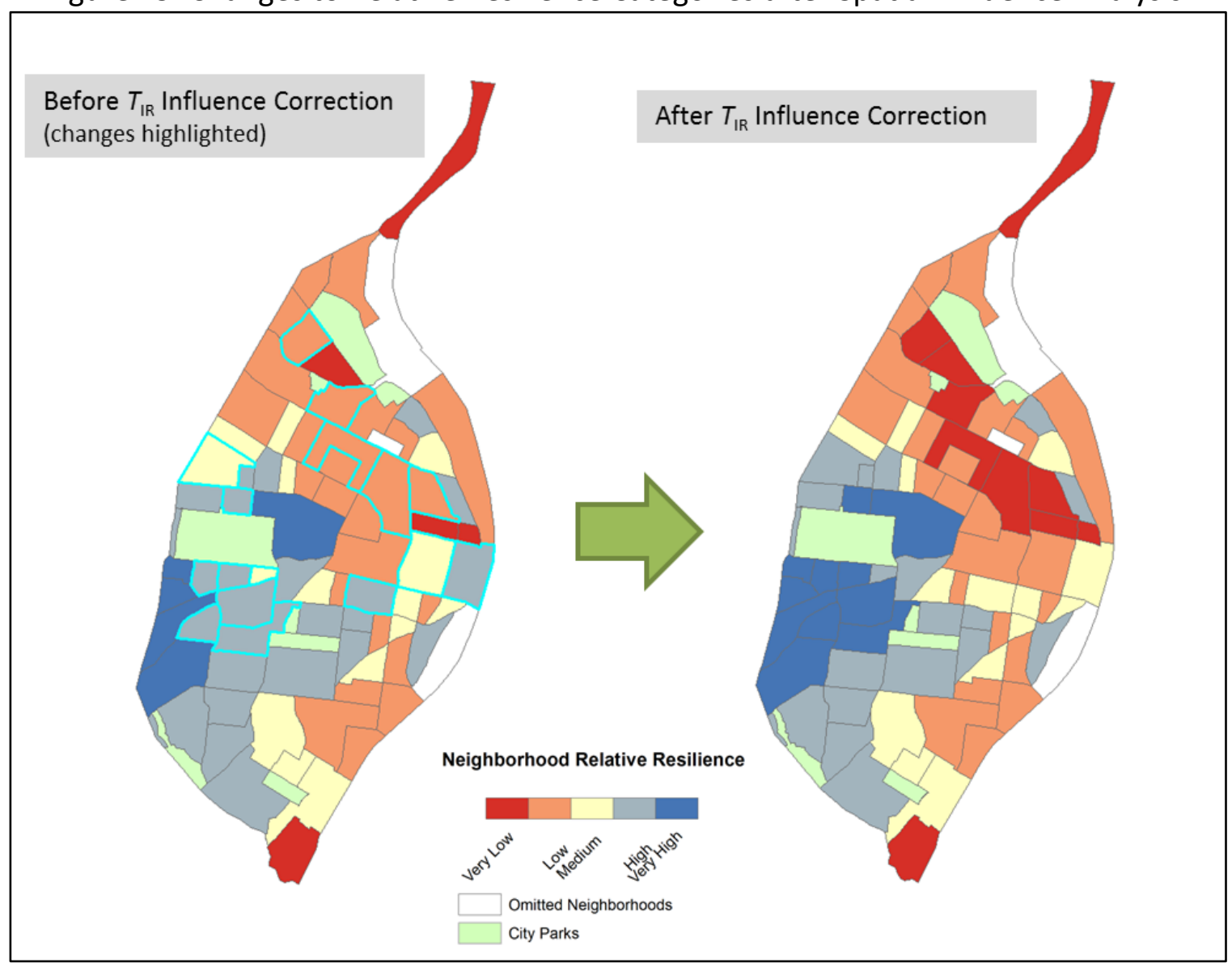


Figure 30. Map and Population Tables of Relative Resilience Categories after Spatial Influence Analysis

\section{Relative Resilience of St. Louis Neighborhoods}
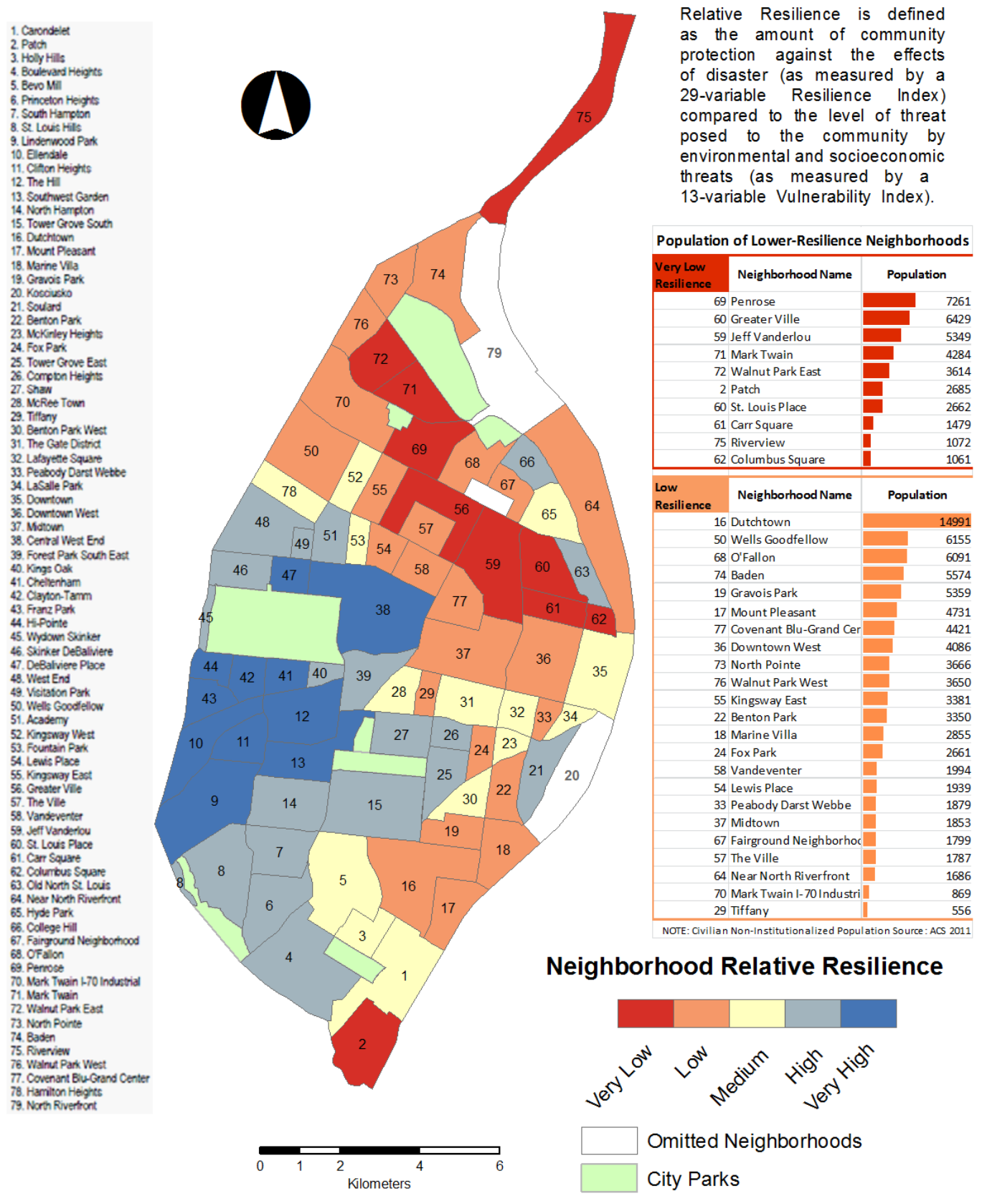
The new categorizations also correlate better with heat death and windstorms request-for-assistance validation datasets. This increased accuracy is expected, because the new categorizations are derived from the spatial qualities of the study area as well as the attributes of the study units. Figures 31 and 32 demonstrate the increased correlation between modelled and actual resilience in the validation datasets.

Figure 31. Correlation of Heat Death Data with Corrected Resilience Model

\section{Correlation of Summer 2012 Heat Deaths With Neighborhood Relative Resilience Categories}
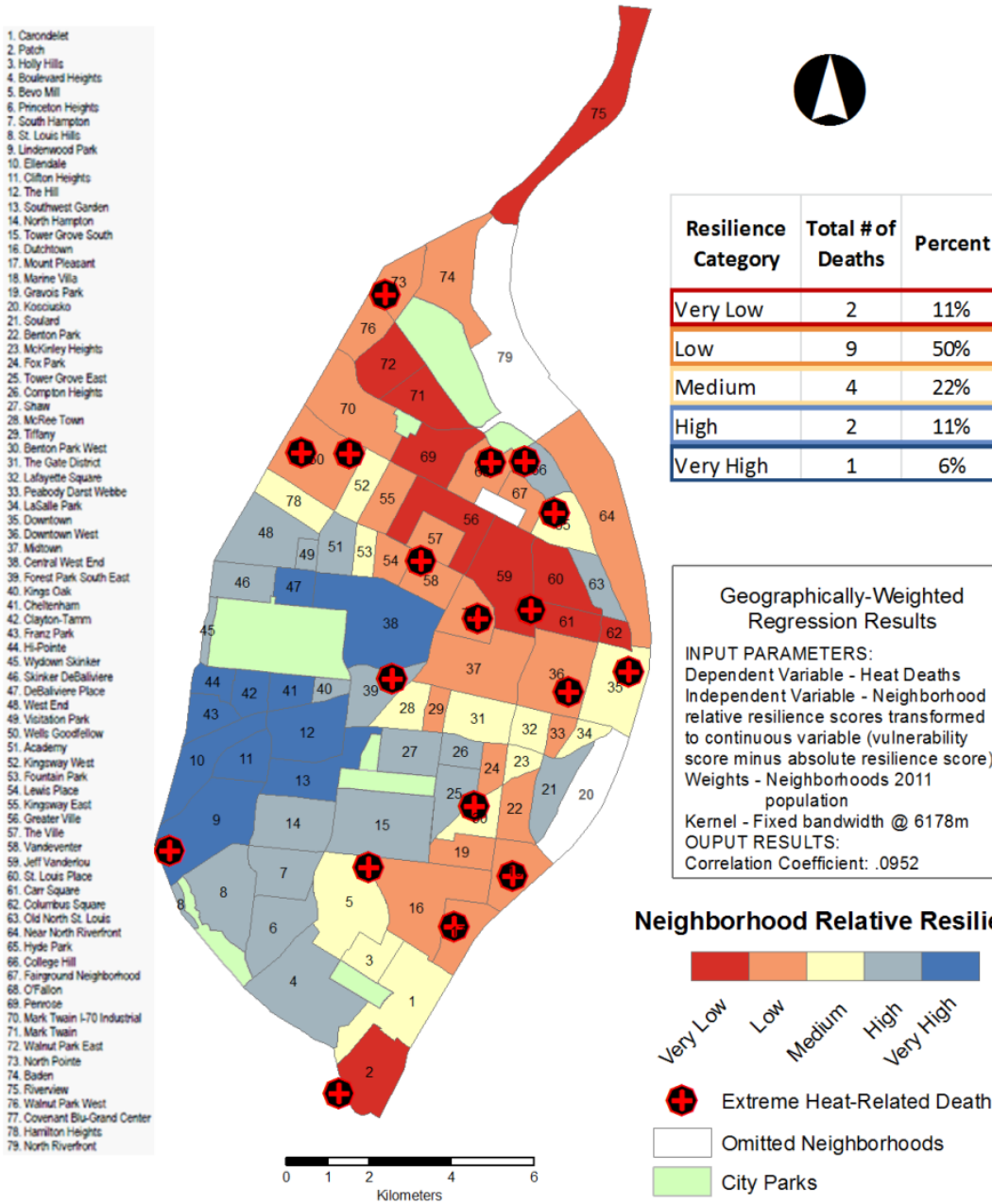

\begin{tabular}{|l|c|c|}
\hline Very Low & 2 & $11 \%$ \\
\hline \hline Low & 9 & $50 \%$ \\
\hline Medium & 4 & $22 \%$ \\
\hline High & 2 & $11 \%$ \\
\hline Very High & 1 & $6 \%$ \\
\hline
\end{tabular}

Geographically-Weighted Regression Results INPUT PARAMETERS:

Dependent Variable - Heat Deaths Independent Variable - Neighborhood relative resilience scores transformed to continuous variable (vulnerability score minus absolute resilience score Weights - Neighborhoods 2011 population Kernel-Fixed bandwidth @ 6178m OUPUT RESULTS:

Correlation Coefficient: 0952

Neighborhood Relative Resilience

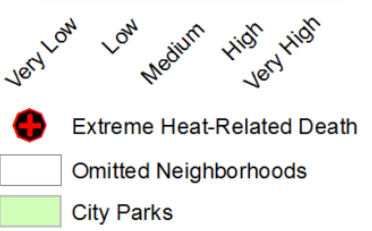


Figure 32. Correlation of Windstorm-Related Requests for Assistance with Corrected Resilience Model

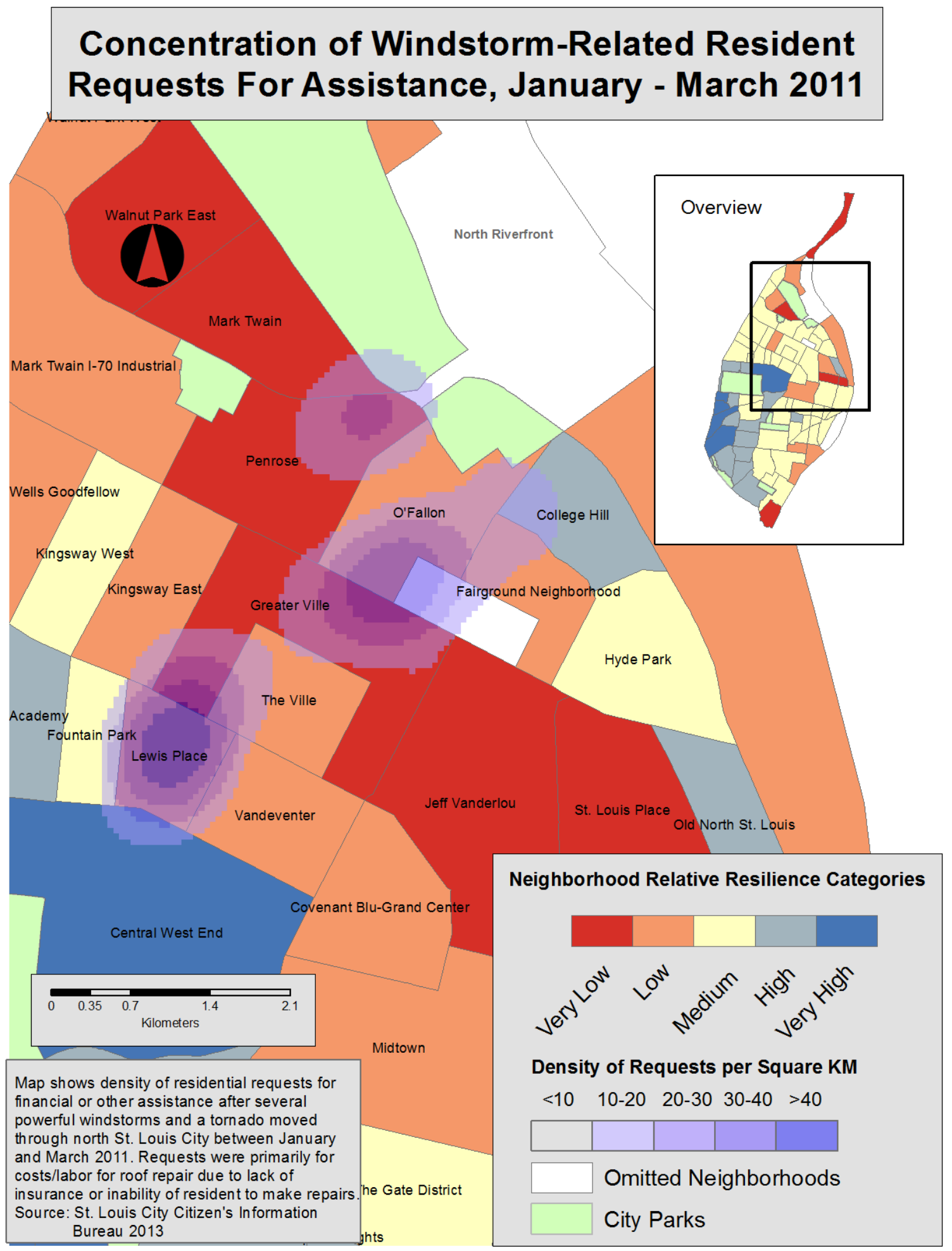


6.6 Comparison of Relative Resilience Model with Alternate Index-Based Model

It is expected that the proposed methods for characterizing community-level resilience 1) will provide better information on resilience-building qualities and processes for the purposes of targeted mitigation (because they account for and explore cause-effect relations among resilience variables), and 2) that the results of analysis using the proposed model (which relies on a knowledge-based system for index construction) will be both significantly different than results of current index-based approaches and also explain observed levels of resilience better than these alternate methods.

This first expectation can only be validated by testing whether the results of the proposed DEMATEL analysis make sense to emergency managers and community leaders in St. Louis, and whether these results can help community stakeholders prioritize mitigation efforts. Emergency management officials from the St. Louis City Department of Public Safety and community organizers including St. Louis Association of Community Organizations (SLACO) have expressed high interest in seeing the results of the current analysis; it is hoped that, eventually, the results of this study will prove useful for these stakeholders and beneficial for St. Louis neighborhoods.

The second hypothesis, however, can be tested by conducting an alternative resilience analysis using data inputs and index construction methods similar to current simple, statistically-corrected additive index approaches. Such an analysis was conducted using a deductive additive index of 17 variables based on Cutter (2008)'s BRIC (Baseline Resilience Indicators of Place) model. Only variables not expected to 
exhibit significant co-linearity in statistical testing were retained (for example, a variable indicating the 'percent population not in poverty' was intentionally omitted due to its expected similarity with another variable, 'percent population employed'). An equal weighting scheme was used for all variables, and standard deviation-based resilience categories were assigned to neighborhoods based on the distribution of equal-weighted index scores. The result of this single index was then rendered on a map for comparison to the output of this study's relative resilience classifications (Figure 33), and tested for its ability to uncover statistically-significant groupings of high- and low- resilience neighborhoods using Local Moran's I (Figure 34).

Figure 33. Comparison of the Present Study's Relative Resilience Classification and the Alternate Classification
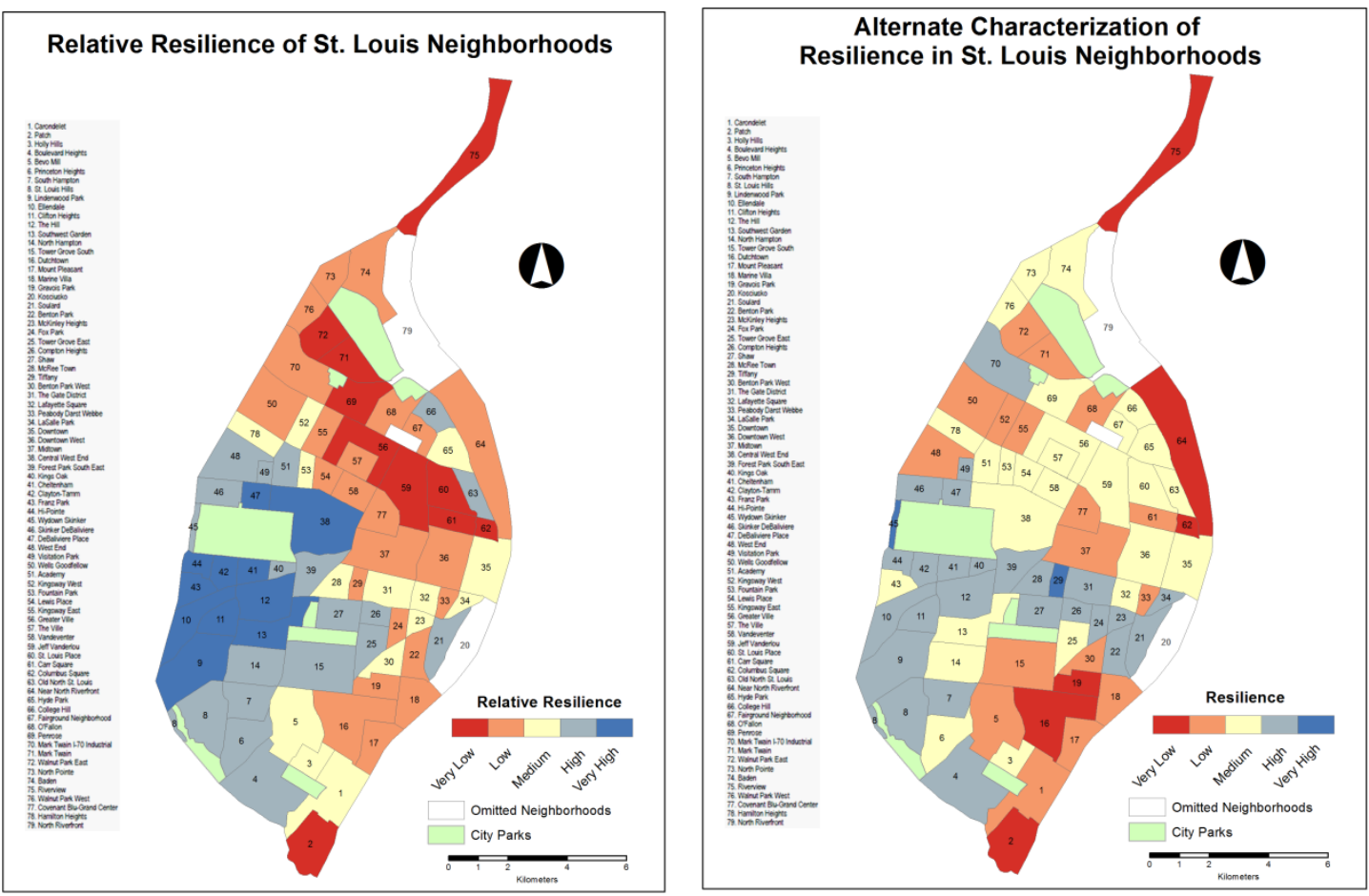
Figure 34. Comparison of Statistically-Significant Clustering of Resilience Classifications (Local Anselin Moran's I) between the Relative Resilience Model and Alternate Model
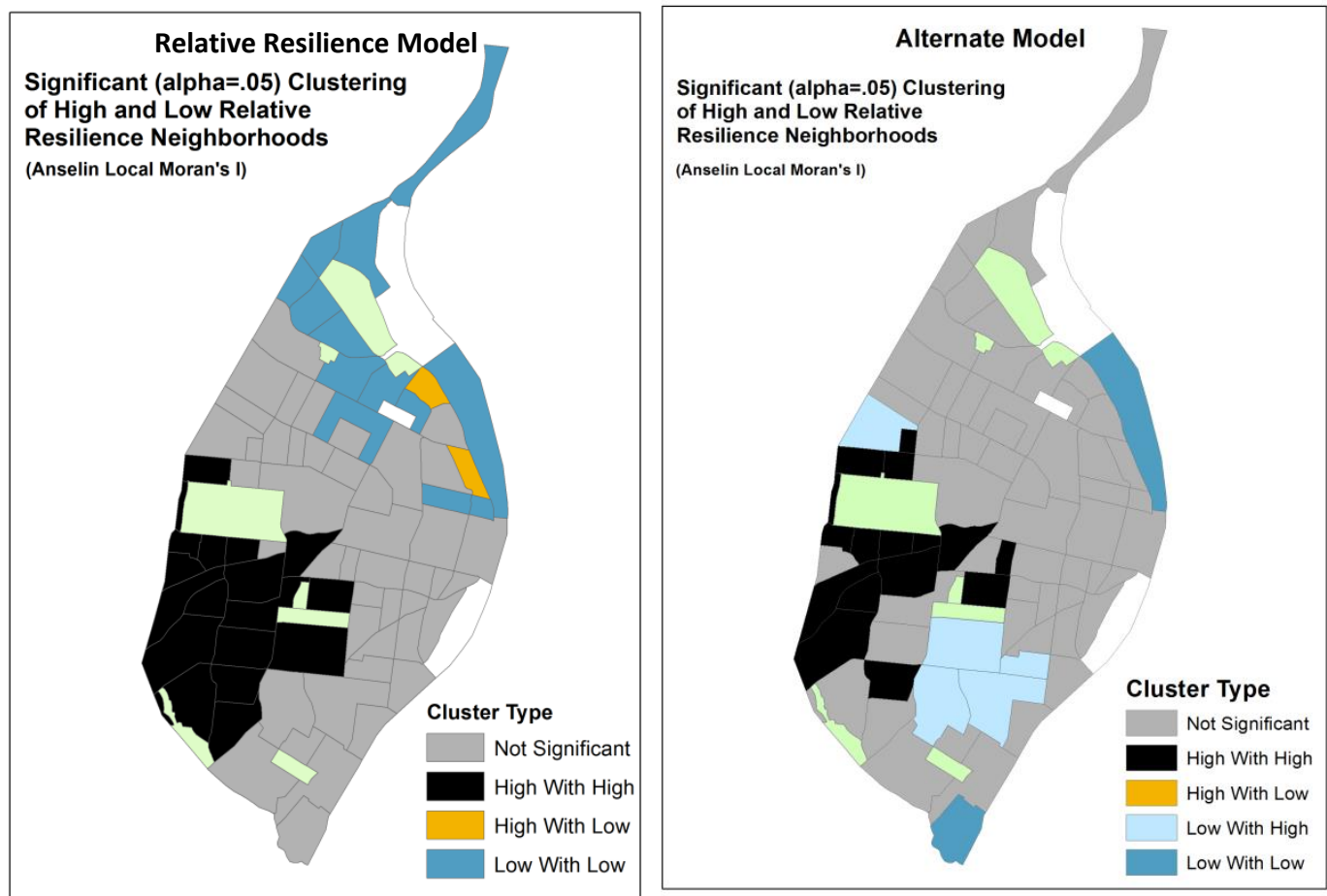

The resulting spatial distribution of neighborhood resilience classifications in the alternate model captures a general characterization of higher resilience in the southwest and mid portions of the city, and lower resilience in the northern and southern portions. This is expected since the variables used in the alternative analysis were mostly representative of individual- or household-level socioeconomic characteristics (as is the case in most index-based resilience research - see section 2.4), and were likely to reflect the obvious differences in small-scale socioeconomic status between certain parts of the City. However, the map of the alternate model suggests that the lowest resilience occurs in the southern portion of the City, which is contradicted by both the proposed model and by the expert and lay reporting of the severity of community threats contained in Understanding Our Needs (STL Health Dept 
2012). In fact, if government official or community leaders were to rely on statistically significant groupings of lower-resilience neighborhoods in the alternate model for mitigation targeting, there is a chance the northern portion of the City could be overlooked, since there are no such groupings there (Figure 34).

When validated against the datasets representing observed levels of community disaster resilience, the alternate model offers significantly less explanatory power than the proposed relative resilience model. In the case of heat death data (2012 heat wave), the alternate model over-predicts the resilience level of some neighborhoods (as many deaths occurred in neighborhoods characterized by the alternate model as having 'Medium' resilience as with 'Low'), and the goodness-of-fit of distribution of heat deaths per resilience classification does not approach the accuracy of the relative resilience model (Figure 35). In the case of resident requests for assistance (2011 windstorms), the alternate model makes little distinction in level of resilience of neighborhoods with high or low concentrations of requests (the majority of all requests, as well as all the area with the highest density of requests, fell in neighborhoods characterized as having 'Medium' resilience in the alternate model - see Figure 36). In the case of population change (post-2008 economic shock), there was no significant difference in explanatory power between the two models after geographically-weighted regression (relative resilience model $r^{2}=.20$, alternate model $r^{2}=.19$ ).

The above alternative index analysis validates the usefulness of an index structured and analyzed based on a local knowledge system (and accounting for spatial 
Figure 35. Coincidence of Heat Deaths and Resilience Classifications Between Relative Resilience Model and Alternate Model
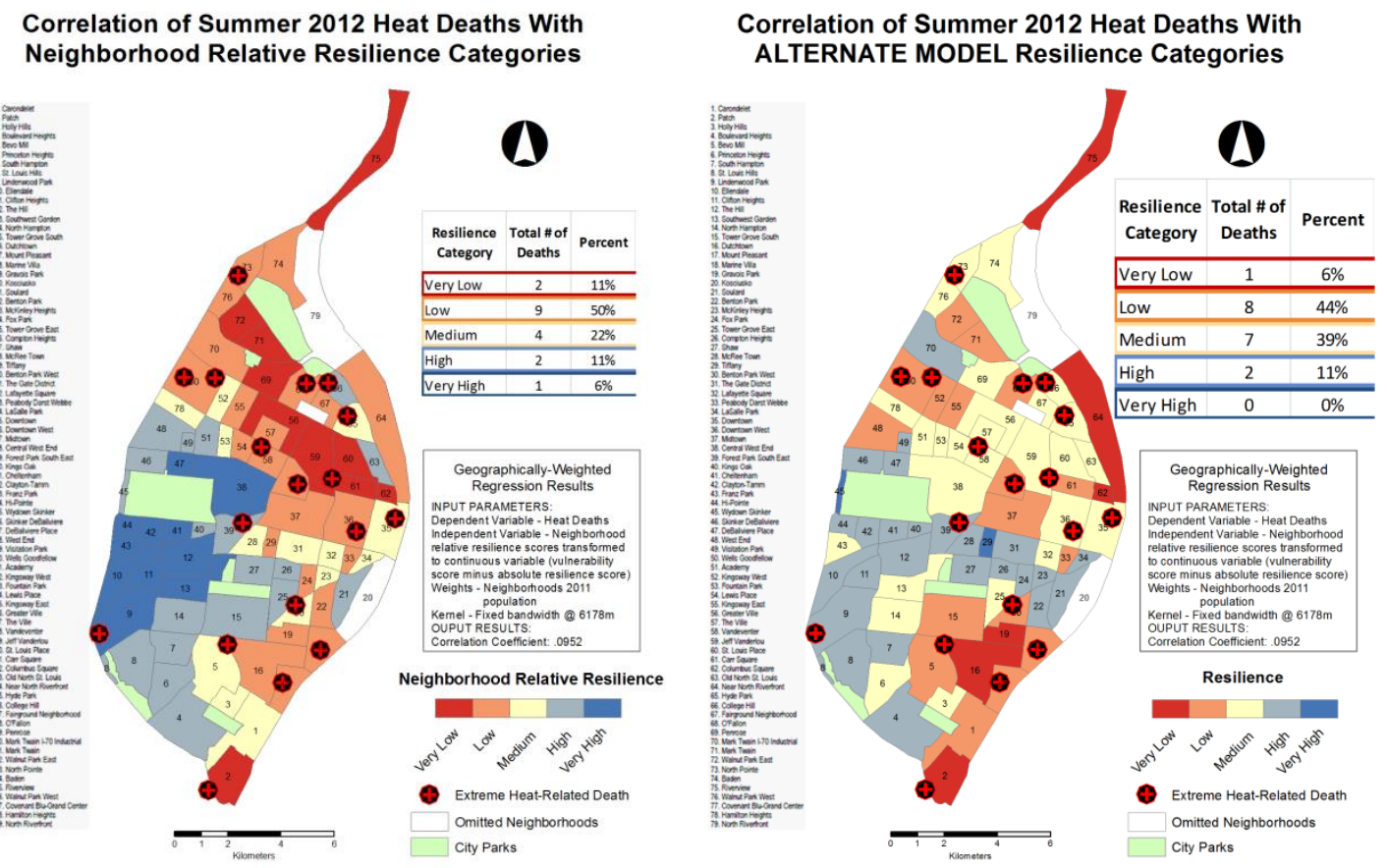

Figure 36. Coincidence of Requests for Assistance and Resilience Classifications Between Relative Resilience Model and Alternate Model
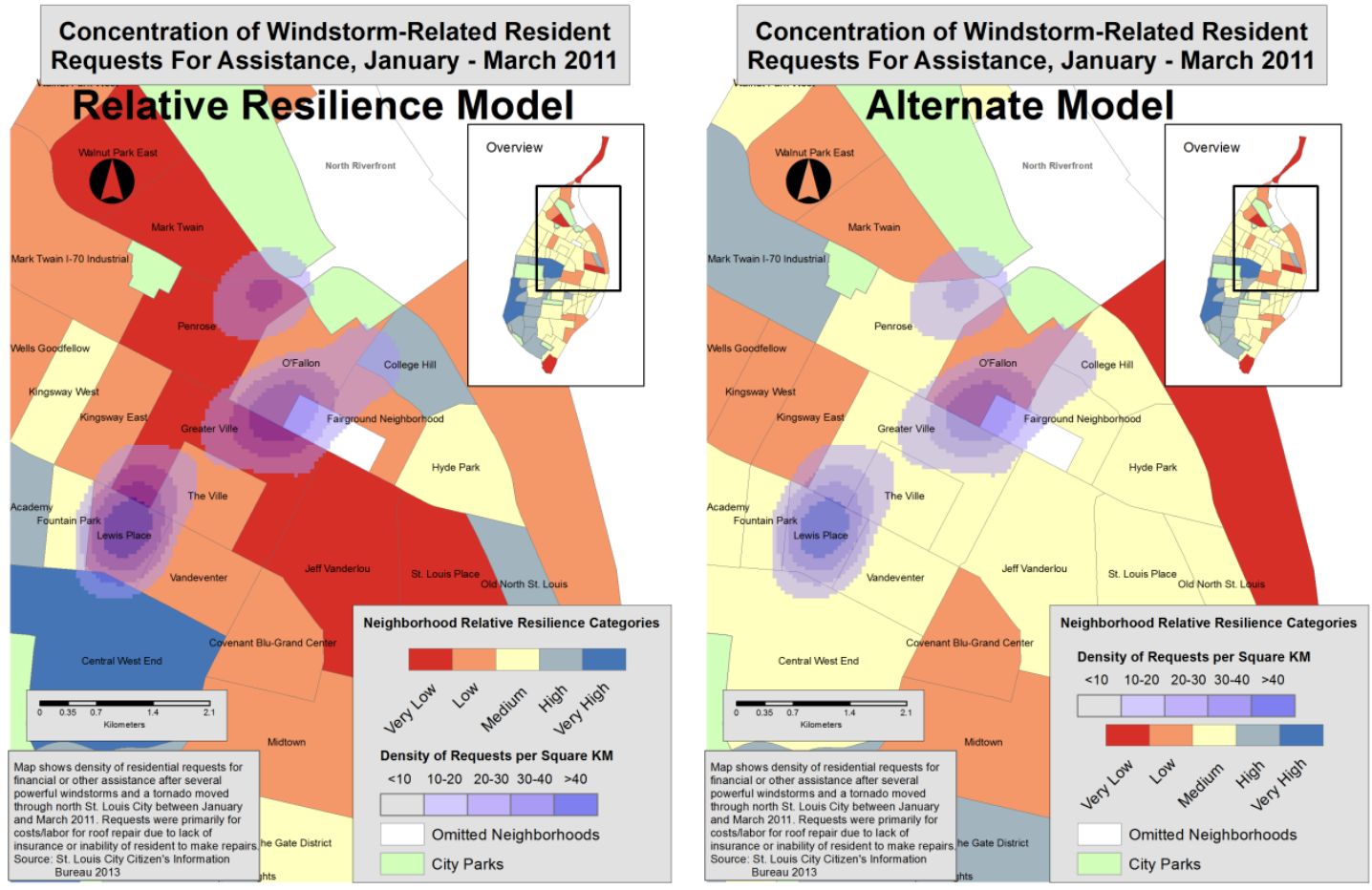
influences) vis-à-vis an approach favoring internal statistical techniques. This is not to suggest such statistical techniques are inapplicable; in fact, they may prove complimentary in a resilience analysis. If, for example, both the knowledge-based DEMATEL method and internal statistics identify certain variables as less informative or relevant to the study, this creates a strong argument for the omission of such variables. The creative integration of data-dependent analysis tools with knowledge-based ones may hold promise for uncovering actionable information in datasets representing complex social processes.

\subsection{The Case for Resilience Model Validation and Correction}

The above discussion, measurement and correction of the validity of the proposed community resilience model was undertaken specifically to improve a model which is meant to be as adaptive and malleable as possible. During the process, issues with weighting sensitivity and neighborhood classification were discovered, and solutions were implemented in order to present the best information to emergency management decision makers. Also, a new method for quantifying the spatial influence among adjacent neighborhoods was developed and utilized to further correct the original relative resilience classification. The relative resilience model was further proved to outperform an alternative community resilience model similar to those used in current literature. Because such corrections are clearly vital to the utility of the model, and would have gone unaddressed if validation were not undertaken, it is surprising that most resilience studies skip this important step (Tate 2012; Tate 2013; 
Wolf et al 2014). While it is unclear why internal or external validation is not widely practiced, the consequences for the intended audience of these studies are clear: important information is lost, and the model's effectiveness is blunted. For this reason, it is recommended that validation of some type is always conducted on index-based resilience models. This process does not need to be thought of as an attempt to conclusively validate or invalidate the model, but rather as an attempt to make it better.

\subsection{On Which Neighborhoods Should Emergency Managers Focus?}

As a result of reclassifications to neighborhood relative resilience categories in Sections 6.2 and 6.5, there more neighborhoods categorized as having low or very low disaster resilience. Ten neighborhoods, with a combined population of 35,896 (11\% of the city total), are classified 'very low'. Twenty three neighborhoods, with a combined population of 85,333 ( $27 \%$ of the city total), are classified 'low'. However, the increase in population and number of neighborhoods identified as high-risk does not necessarily translate to a more difficult mitigation task for emergency managers. The spatial characteristics of clusters of high-risk neighborhoods, as well as the spatially influential nature of identified resilience characteristics, may actually make mitigation efforts more efficient. This is because the effects of disaster mitigation policy - which may include efforts to boost any of the resilience indicators listed in Table 8 by government and community leaders - can be expected to attenuate into surrounding neighborhoods due to feedback relationships inherent in complex social systems. Well-chosen mitigation tactics (see Section 6.7 for this study's recommendations) directed at the contiguous 
cluster of 'very low' resilience neighborhoods in North St. Louis may positively affect surrounding neighborhoods identified as having 'low' resilience. There is also reason to believe that the strong resilience-boosting processes identified in central city neighborhoods may positively affect the lower-resilience neighborhoods to the North.

Since this study used neighborhood population to help define their spatial influence, it makes sense to also recommend that emergency mangers and community leaders focus their attention on the higher-population neighborhoods. Of course, when analyzed by population, there may be neighborhoods classified as 'Low' or even 'Medium' resilience which deserve attention due to their high population. Such is the case in St. Louis for the Dutchtown neighborhood - its population of 14,991 make it by far the most populace low-resilience neighborhood, and should be considered for mitigation. To help with the spatial identification of high-population neighborhoods visà-vis their resilience categories, it is useful to create a 3D visualization of St. Louis where lower-resilience neighborhoods are extruded vertically by their population (see Figure 33). 
Figure 37. Example 3D representation of St. Louis neighborhood population and resilience categorization

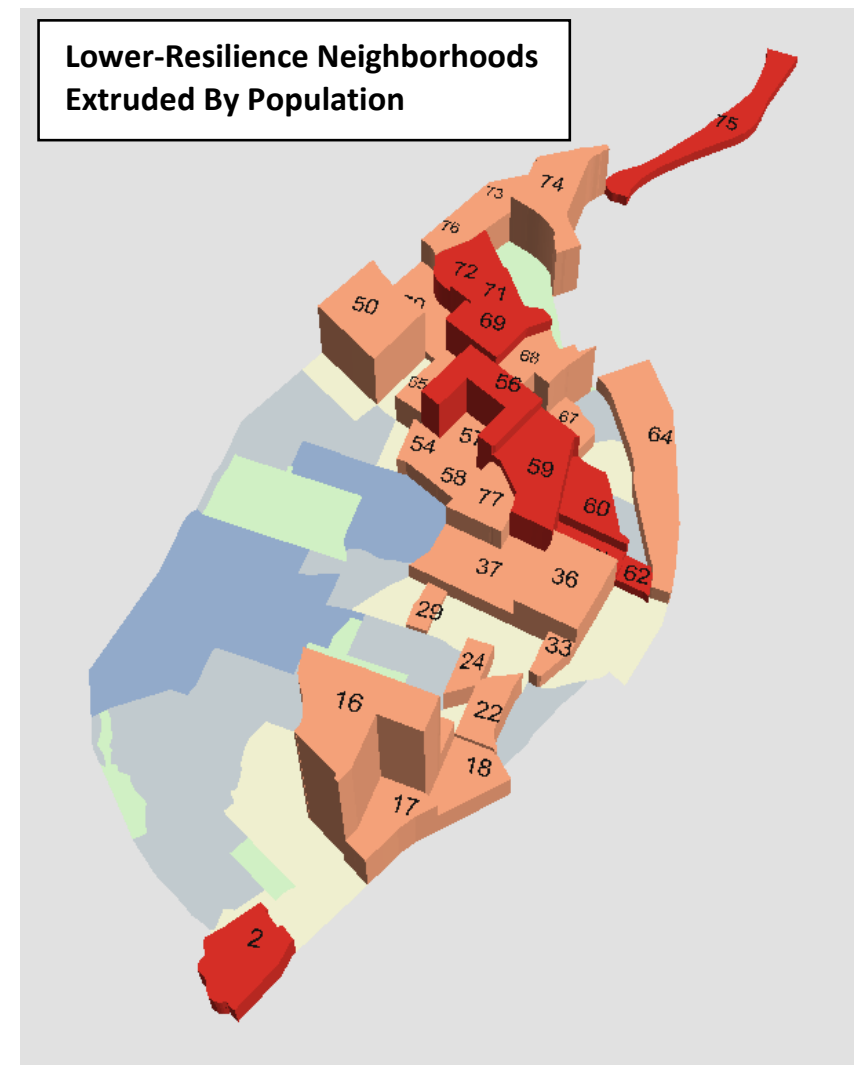

\subsection{What Community Characteristics Should Emergency Managers Target?}

Spatial analysis techniques have been used to lessen the uncertainty of which neighborhoods should be given priority attention by emergency managers and community leaders. However, without a way to distinguish the relative importance of the large set of resilience indicators used in this study, these decision makers would be very uncertain as to which of these community qualities and processes to address in mitigation efforts. The results of the earlier DEMATEL analysis provide compelling evidence for which indicators are of a more causal and influential nature and, as such, are expected to strengthen overall community resilience more efficiently than others. At 
the end of DEMATEL, the total direct-indirect relationship matrix T (see Section 3.4) is used to calculate a position value $s+i$ (which measures how dependent an indicator is on the values of other indicators, similar to Pearson's $r$ ) and relation value s-i (which measures an indicator's causal influence on others) for each resilience indicator. When $\mathrm{s}+\mathrm{i}$ and $\mathrm{s}-\mathrm{i}$ are plotted on a graph, a quadrant overlay can be used to visually distinguish indicator which fall into 4 general categories, as depicted in Figure 19.

These quadrants and their associated interpretation are:

1) High Position / High Relation Values - indicators with the highest influence on other indicators, and of a causal nature

2) Low Position / High Relation Values - indicators which exert high influence but do not seem to be themselves influenced much (independent variables), and also of a causal nature

3) Low Position / Low Relation Values - indicators which influence others only weakly, yet seem to be mostly controlled by other indicators, making them of an effect nature

4) High Position / Low Relation Values - indicators which exert little or no influence on others, and seem to be merely effects of other indicators

These quadrants are listed in order of their constituent indicators' relative influential power. Increases in quadrant 1 indicators (high s-i/highs+i) are expected to have the highest positive influence on all other indicators. This effect may be magnified for quadrant 1 indicators because they are also expected to be receptive to positive feedback sent out by other influential variables in other quadrants. Increases in quadrant 2 indicators will have the same type of initial influential effect as quadrant 1, but because they are more independent from received effects, these indicators probably will not benefit from positive feedbacks from others. Increases in quadrant 3 indicators cannot be expected to have much of a positive effect on other indicators, yet 
they are receptive to received influence, so they may be boosted by increases to indicators in quadrants 1 and 2. For quadrant 1 indicators, there is little evidence that increases to their values would have any effect on other indicators, yet their values are also highly dependent on those of other indicators; it can be assumed that quadrant 1 indicators will change according to the aggregate influence of all others, but will not exert any meaningful influence.

The qualities of the above quadrant intensify toward the outer corners of each quadrant (representing more extreme values), and diminish toward the center, so that indicators nearer to the midpoint may not significantly demonstrate any of the associated qualities. Based on these characterizations, quadrant 1 indicators should be the first choice for targeting in resilience mitigation strategies, since they are highly influential, of a causal nature and receptive to feedback. Interestingly, most of the indicators in this quadrant included most of the diversity-related, community-level characteristics, such as N9-Occupational Diversity, N10-Business Size Diversity, N6Income Equity, and N7-Racial Diversity. Other indicators significantly inside quadrant 1 include N11-Innovative Potential, G9-Institutional Capital Development, N3-Community Involvement: Active Neighborhood Business Association, and 12-Educational Attainment. There are no indicators that can be identified as of the quadrant 2 type (highly independent variables), yet quite a few of the quadrant 3 type (those that exert less influential but are receptive to incoming influence). These indicators are still highly relevant to mitigation, yet should be considered secondary to those in quadrants 1 and 2. Quadrant 3 Indicators nearer to the extreme end include G3-Government Extreme 
Temperature Mitigation Effort, G4-Government Emergency Food Mitigation Effort, G5Government Gender-Based Emergency Mitigation Effort, G7-Institutional Emergency Health Mitigation Effort, I1-Civic Participation, and I7-Elderly \& Disabled Population Connectivity. There are only two indicators which fall in quadrant 4 (highly dependent, not influential) - thankfully so, because these indicators (N4-Community Identity: Historic District and G6-Government Maintained Multi-Purpose Space) are not to be considered very relevant to disaster mitigation and probably should not be included in future research (Hiete et al. 2012).

The validity of the DEMATEL method is certainly bolstered by the above set of quadrant 1 variables, which may rightly be considered true benchmark traits of strong and resilient communities. However, they seem to also be some of the most difficult to address and possibly the slowest to respond to improvement efforts. Yet, from the emergency manager's perspective, it may be encouraging that the most sought-after traits for urban residential communities may also be considered crucial protections against the effects of extreme events. This is because a very broad range of municipal government policy, institutional attention and community action are already directed at improving these particular conditions. At the government level, a certain amount of responsibility for the growth of these indicators is institutionalized across common municipal departments such as public health, education, economic development, and others. Therefore, where community resilience is judged to be low, it should not be considered the fault of a particular government office or community leader, nor is it the responsibility of any one entity to remedy. In fact, traditional top-down mitigation 
which relies on government action will probably not be sufficient to address an issue such as community resilience, an attribute which is both driven by and the result of many interacting, multi-scale processes involving many types of actors, both social and environmental. Resilience is, if nothing else, complex. Attempts to increase resilience at the community level will probably only be effective if undertaken and supported equally at the different scales complex processes operate (for example, at the household, neighborhood, and government level).

This is not to suggest that government-led mitigation efforts are inherently less influential, or that the above DEMATEL analysis of indicators leaves government emergency management officials in St. Louis with few options to target. Even though no 'Government' indicators are present at the extreme end of quadrant 1, there are indicators near the center of the graph (variables with uncertain levels of influence/dependency but still may be highly relevant to resilience) which are quite government-specific, such as G1-Official Ward Association and G2-Government Economic Development Effort. Also, most of quadrant 3, as noted above, contains the type of brick-and-mortar emergency relief services which governments are well-suited to create, fund, and maintain.

\subsection{Model Modifications, Alternative Methodologies and Future Directions for Community Resilience Research}

The methodologies used in this study were selected in part based on their recognized adaptability and customizability, which was necessary since there are no complexity-science oriented measurement tools designed to be applied to resilience 
research, and only a few hints of the applicability of the chosen tools. With little to help guide (and nothing to constrain) this effort, many of the variable choices, index structures, tool parameterizations and other methodological choices were based on knowledge of the study area, examination of the data, and related examples in the literature. Therefore, there are plenty of opportunities for modification of the proposed techniques.

First and foremost, it must be noted that the spatial unit chosen to represent 'community' in this study was by no means meant to be normative. Based on a combination of the author's knowledge and local expert reporting of the social characteristics of the city of St. Louis, individual city neighborhoods were selected to represent communities. Neighborhoods were primarily chosen because of St. Louis' unique history of neighborhood self-identification, and therefore the reasonable expectation that residents within them would come together to solve problems in the event of a disaster. However, there are a wide variety of other ways in which a city can be partitioned into areal units of analysis, both larger and smaller than city neighborhoods, which would fit this definition of 'community'. Therefore, it is highly encouraged that selection of spatial units in future community resilience research be similarly guided by the unique characteristics of the area to be studied, not on any universal spatial definition.

Next, the choices of resilience indicators, while reflective of many multi-scalar qualities and processes which make up community resilience, are by no means exhaustive. There are other indicators in the current literature which could have been 
applied (but were omitted due to data availability), and it is expected that additional promising indicators of resilience will continue to be proposed as the academic debate evolves. It is important to also point out that variables which could be chosen to represent these indicators are quite diverse, with many other possibilities than those chosen here. Further, an improvement to the indicator/variable selection methodology would be to find variables which represent each indicator at the household, neighborhood and government scale. In this thesis, this was accomplished to a limited extent (for example, variables related to the 'Civic Involvement' and 'Political Engagement' indicators were found and used for each scale of the resilience index), but increased scalar coverage for each indicator would be expected to further strengthen the model.

Though the process of weighting variables and sub-indices received careful methodological scrutiny and used a knowledge-based approach for better place-specific accuracy, many modifications and improvements could be made to this step. First, only one analyst (the author) contributed the AHP assessment to define vulnerability and resilience variable weights. Commonly, in the business management context that AHP is often used, multiple analysts contribute an assessment, and the multiple results are averaged together before defining weights (Aczel and Saaty 1983). It is expected that a more diverse fusion of expert judgments of the relative importance of various vulnerability and resilience indicators would benefit this type of study. Undertaken in quite a different way than individual variable weighting, sub-index weighting is judged to be the most subjective step of the index construction methodology. Since there are 
generally few sub-indices used in disaster vulnerability/resilience studies, it may not be necessary to utilize a knowledge collation tool such as AHP in order to set these weights; however, as Tate (2012) shows, these weighting choices are likely to have the highest effect on model output. While a balanced and somewhat conservative weighting scheme was applied to sub-indices (and to the internal sensitivity validation stage), it cannot be assumed that local emergency managers or community leaders would always choose such an approach. It would therefore benefit further study to experiment with the effect of more extreme sub-index weighting schemes on the model, especially to show differences in final community relative resilience classifications for different weighting schemes.

The proposed method to construct separate vulnerability/resilience indices and combine them categorically using a confusion matrix is a highly customizable process. In this study, since neighborhood vulnerability and absolute resilience scores were normally distributed, grouping these neighborhoods by standard deviational categories was viewed as appropriate. There are, of course, several other parametric and nonparametric options for defining categories based on the observed distribution of vulnerability/absolute resilience scores. Experimentation with categories based on geometric mean, equal interval or natural data breaks (Jenks and Caspall 1971) could yield different results which may be more appropriate for other types of observed distributions. Different rules for defining categories must be made in the confusion matrix, this time for the sake of assigning relative resilience classifications to communities. Again, this is a subjective choice, made in view of the distribution of 
community vulnerability/absolute resilience category associations and considering the information requirements of the intended audience. As was demonstrated in this thesis, the ability of the model to convey information about certain neighborhoods and its goodness-of-fit with validation data depends on how relative resilience is classified in the confusion matrix. It is recommended that effects of the chosen relative resilience classification scheme be carefully considered in future research.

Finally, while DEMATEL analysis is judged to be a highly effective tool for providing information on indicators likely to be most effective in raising overall community resilience, the effectiveness of its implementation depends on the quality of its original knowledge-based indicator analysis. As in AHP weighting, only one assessment of variable inter-influence was utilized for the DEMATEL analysis, yet it is expected that a collation of multiple, diverse expert and lay opinions be attempted in future research. This is not an easy task, since, in this thesis, a $29 \times 29$ matrix had to be populated with quantified $i \rightarrow j$ judgments. If multiple matrices are to be sourced for a particular study area, a high level of community buy-in for the study, and perhaps focus group sessions, would be necessary. Nonetheless, without a DEMATEL-like analysis, emergency managers and community leaders would be at a loss to subjectively and simultaneously prioritize a very large and complex set of indicators for mitigation purposes.

As has been mentioned, disaster resilience is a relatively new and recently 'hotbutton' issue in academia (Norris et al. 2008, Reghezza-Zitt et al. 2012). As such, methodologies proposed for examining the concept and measuring it at the community 
level are not quite mature, and in fact most current efforts utilize tools, such as additive indices, which were originally designed for different applications. This study used a hybridized and modified additive index methodology in order to integrate concepts from complexity science-inspired disciplines. This fusion of methods was undertaken not only because it was considered plausible but because it was expected to result in better understanding of resilience than studies based on either pure linear indices or the abstract heuristic techniques of complexity science. However, there are probably conceptual frameworks and methodologies completely unrelated to indices or heuristics which can offer explanations for some of the facets of community resilience. Many opportunities for trans-disciplinary linkage and methodological pluralism are expected to exist than those laid out here. As mentioned, observation-based empirical research which studies community socioeconomic processes as they change through time may be considered highly promising. Also, network-based models of the interaction of important community actors, resources and power relations may hold strong explanatory power for how clusters of high and low resilience came to exist, and how outcomes of disasters are shaped by pre-existing socioeconomic relations. Since the concept of community resilience is often portrayed as baffling, yet at the same time highly important and of a high research priority, there are bound to be brand new techniques developed to measure it. Just as the development of fuzzy logic was inspired by the inability of positivist, linear-oriented methods to explain social systems in the 1960s and 1970s (Zadeh 1976), current frustration with the concept resilience may spur important methodological innovation in the future. It is important to look out for these 
developments, and actively integrate them into research. Efforts to measure community resilience would benefit from being as adaptive, opportunistic and creative as the components of the concept itself. 


\section{CHAPTER 7. CONCLUSION}

In this thesis, it is recommended that future community resilience research recognize and take into account the inherently complex nature of resilience as it has been described by various disciplines. Here, this complexity was addressed on several fronts. The larger concept of community resilience was deconstructed into a multiscalar framework to allow for balanced analysis of resilience-building qualities and processes at the levels which they operate. Promising knowledge-based techniques for quantifying dynamic influences and cause-effect relationships in the chosen resilience indicators were operationalized to set indicator weights in an index and to provide information for the most effective mitigation strategies. A new method was introduced for estimating positive and negative resilience feedback relationships among nearby communities based on the interacting spatial characteristics of those communities.

As applied to neighborhoods in the city of St. Louis, Missouri, the resulting community resilience model identified significant clusters of high and low resilience neighborhoods, which were validated against datasets representing observed outcome of extreme environmental and socio-economic events. Though successful in explaining some aspects of community disaster resilience in St. Louis, this research is not intended to endorse a specific set of optimal resilience measurement tools or suggest the universal applicability of the chosen methods. Rather, the intent of this research was to show that much of the uncertainty and frustration which has defined the recent 
academic debate over the concept of population resilience can be alleviated by a shift away from linear-oriented conceptualizations and statistically-dependent measurement methods in favor of any approach which attempts to integrate qualitative perspectives, local knowledge and nonlinear system modelling procedures.

Evidence that this shift is presently occurring within geographic perspectives of community resilience is increasing, as recent studies have: taken into account the multiple spatial and temporal scales of resilience-building or resilience-degrading community processes (Hoeflehner 2014; Lew 2014; Rodina 2014), adapted theories of complex socio-ecological systems to conceptualize resilience (Bitterman and Bennett 2014; Engie and Quiroga 2014), argued for more local-scale, place-specific characterizations of community resilience (Chan et al. 2014; Lazarus 2014), and utilized local expert and lay perspectives of both hazards and community protections against those threats (Bergren 2014; Kumari and Frazier 2014; Leichenko and Solecki 2014; Walsh-Dilley et al. 2014).

With the increased multi-disciplinary attention afforded to the subject, the study of resilience may be entering a renewed period characterized by a plurality of methodological treatments and a fusion of diverse knowledge sources. Local emergency management decision makers and community leaders stand to benefit from the lessened uncertainty that such academic conversations can provide. 
APPENDIX A. ENVIRONMENTAL HAZARD VARIABLES
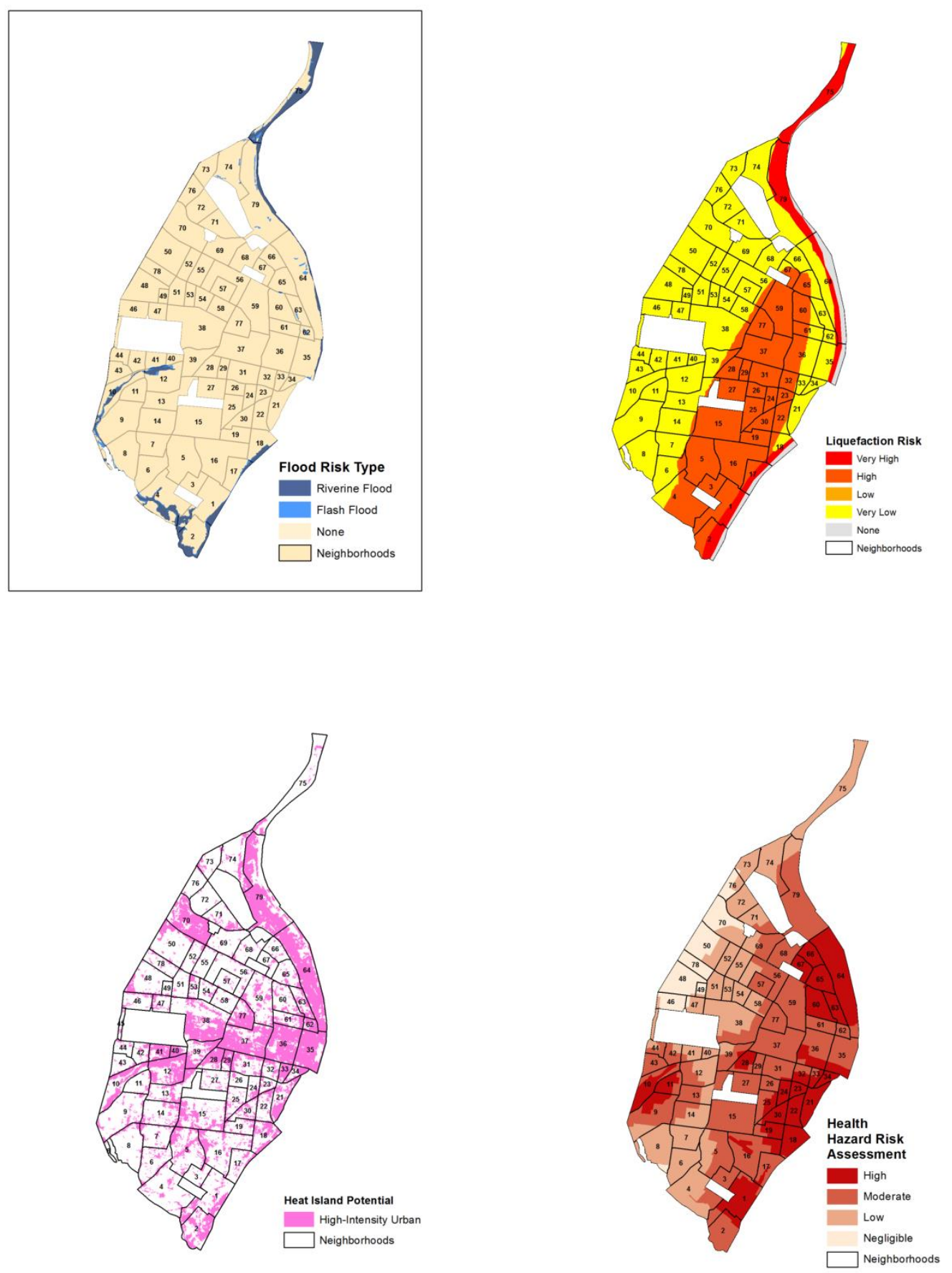


\section{APPENDIX B. INDICATORS OF VULNERABILITY}
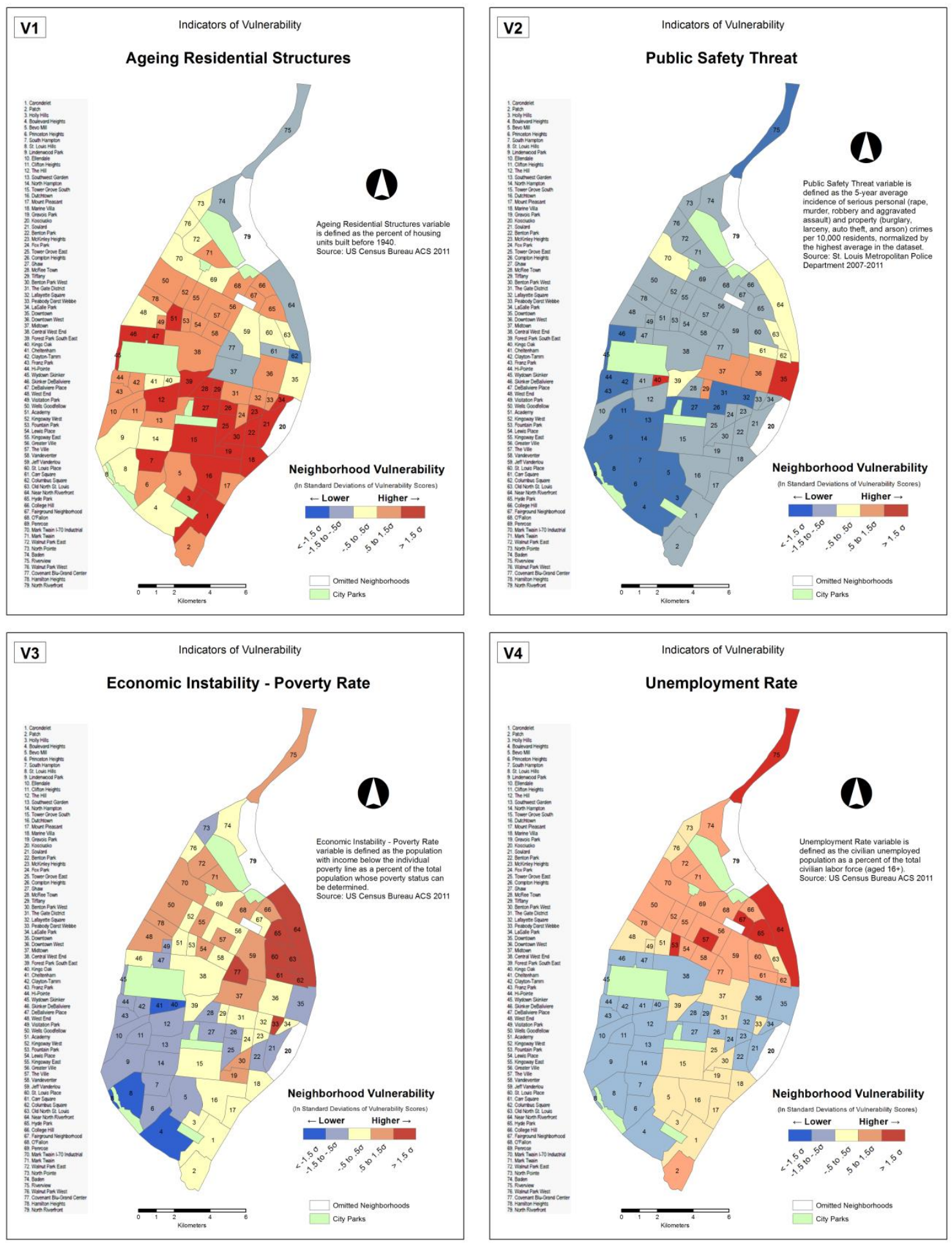

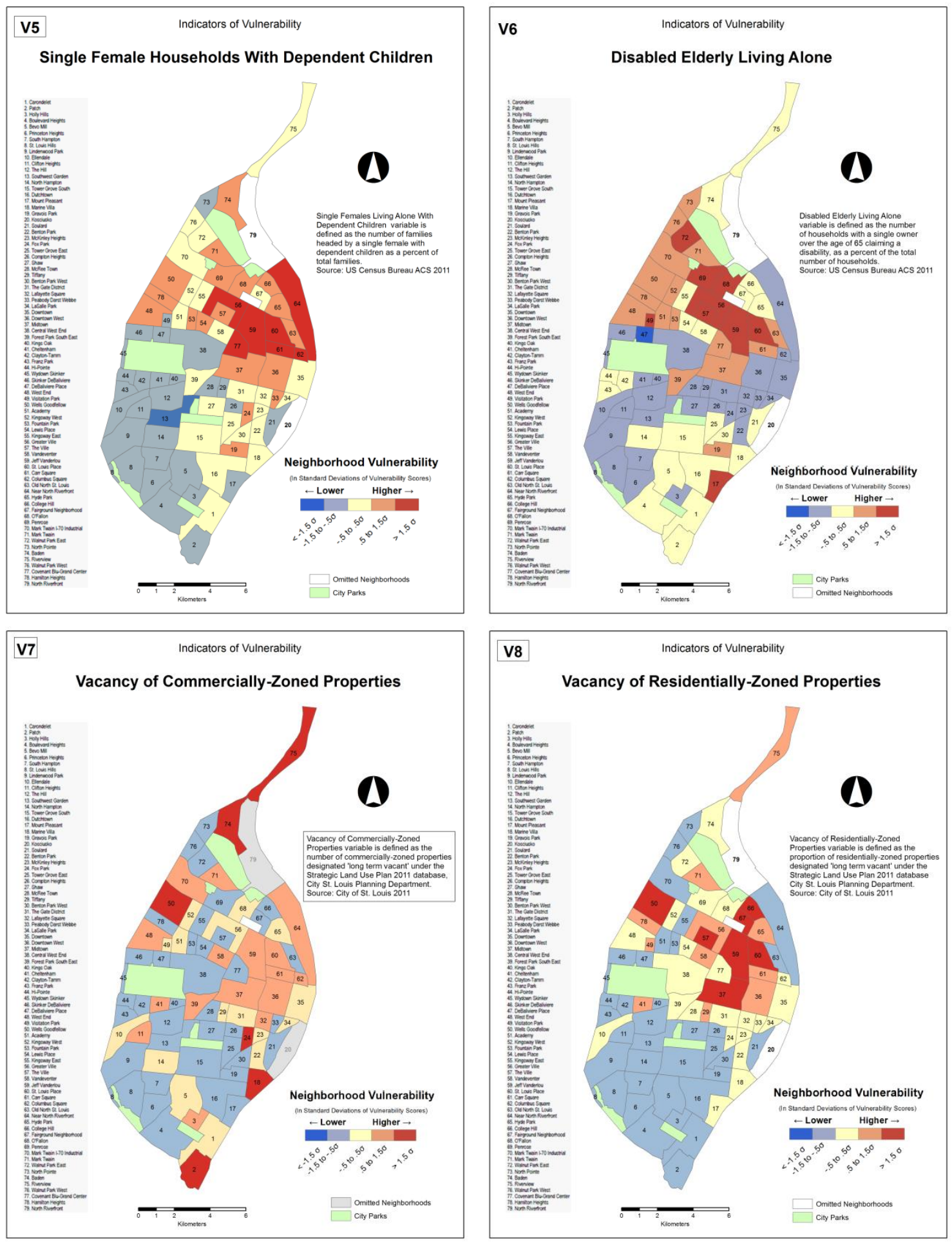

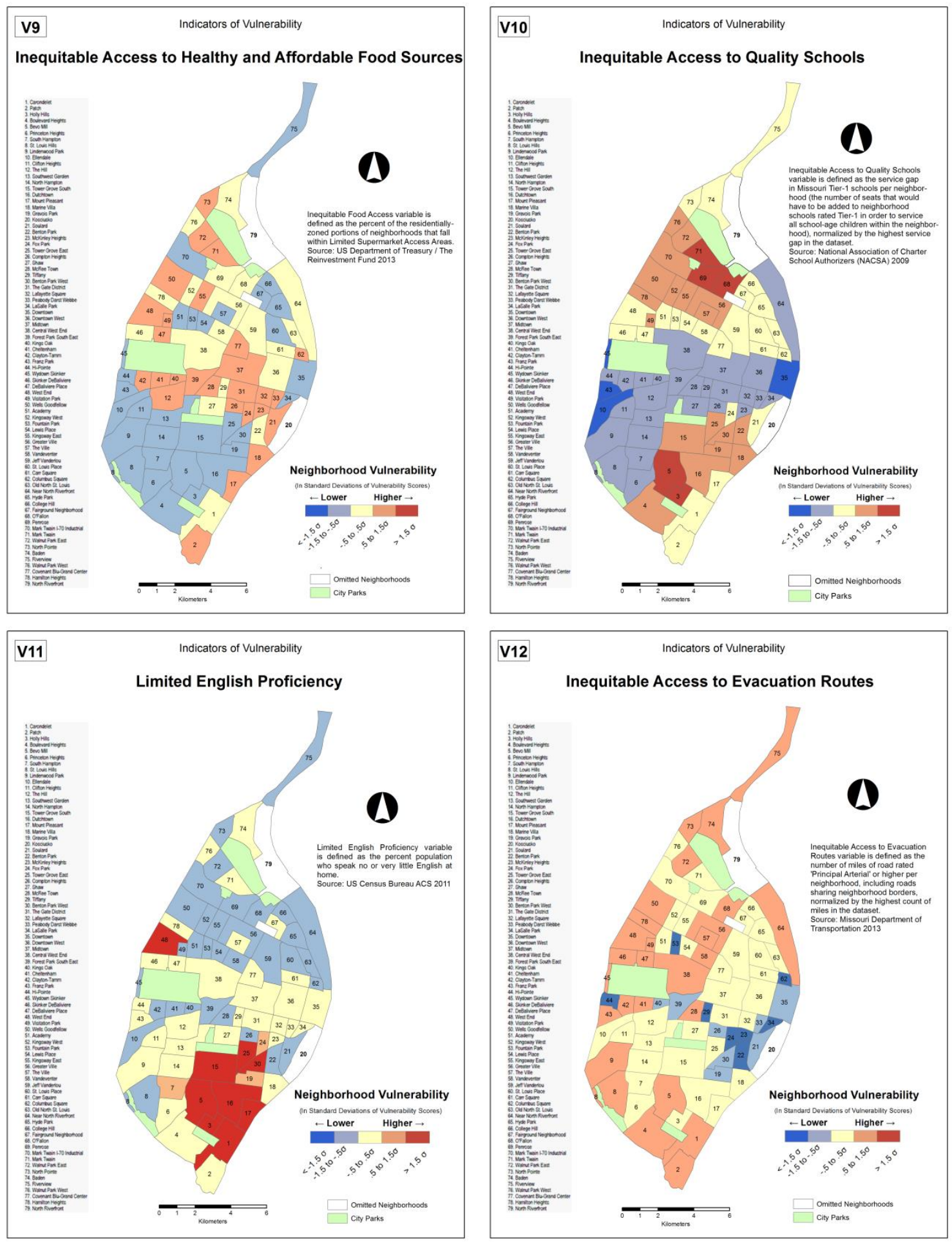


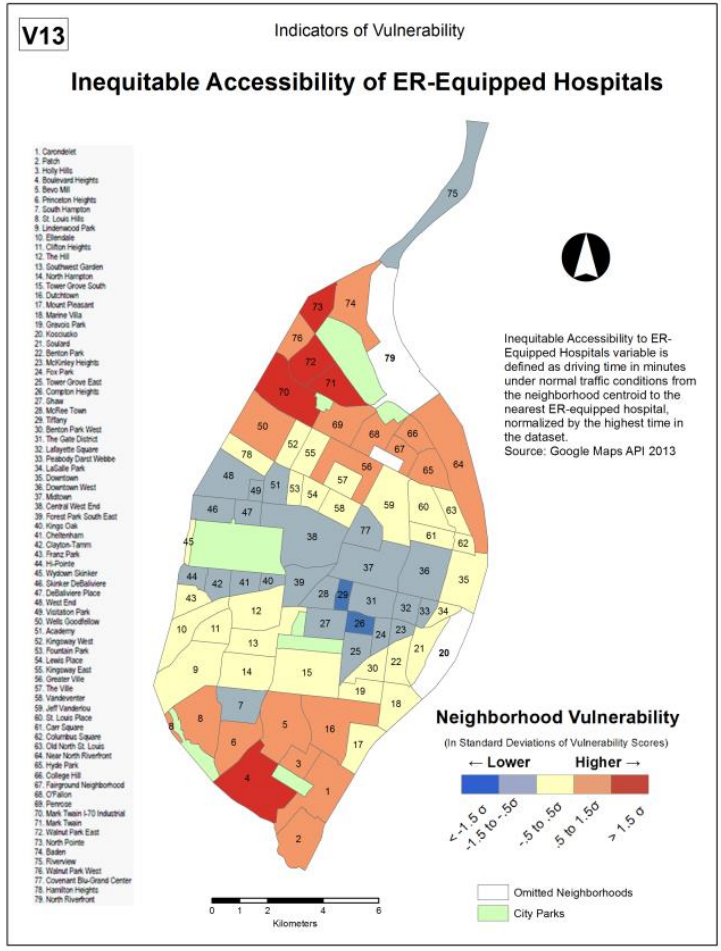




\section{APPENDIX C. INDICATORS OF RESILIENCE}
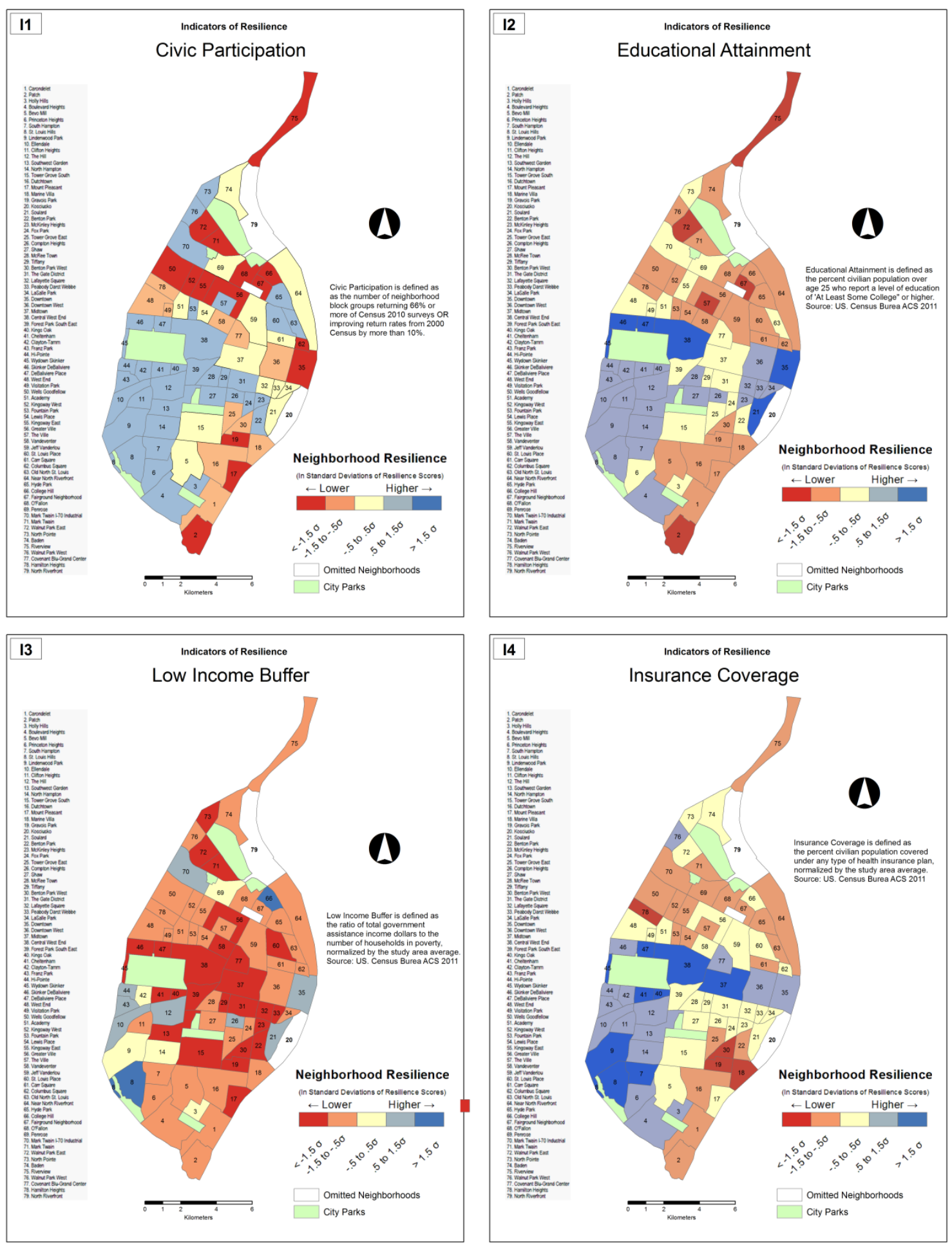

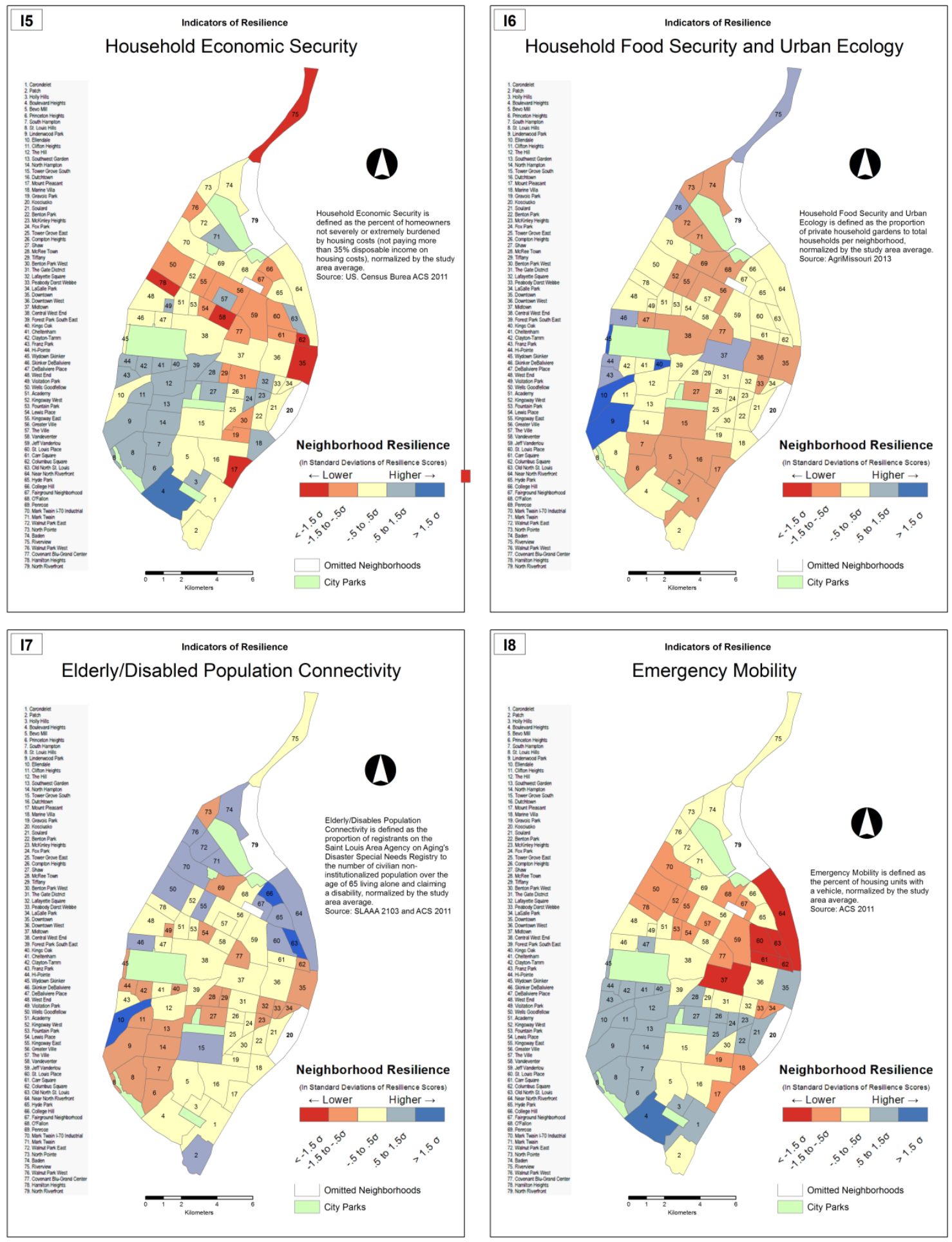

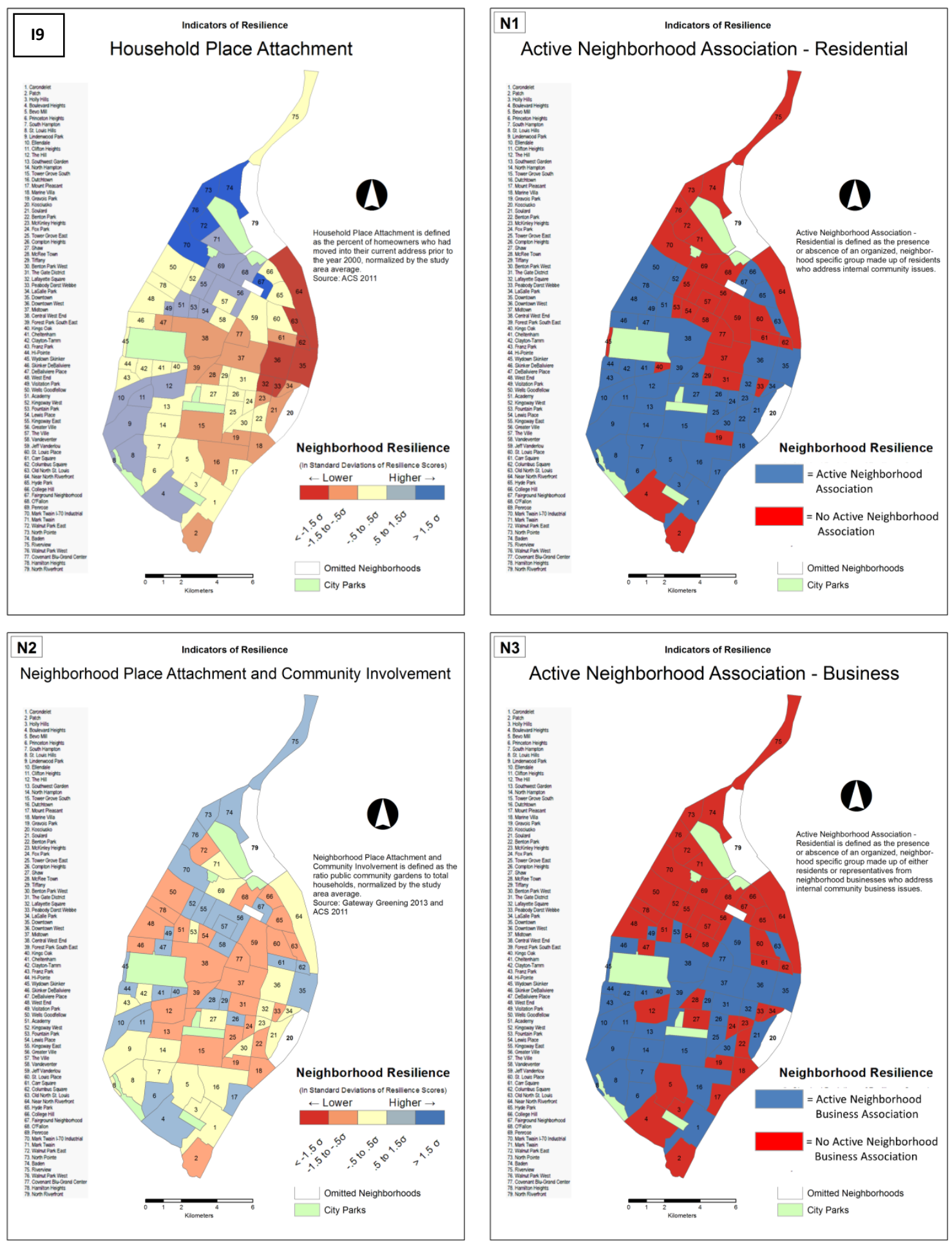

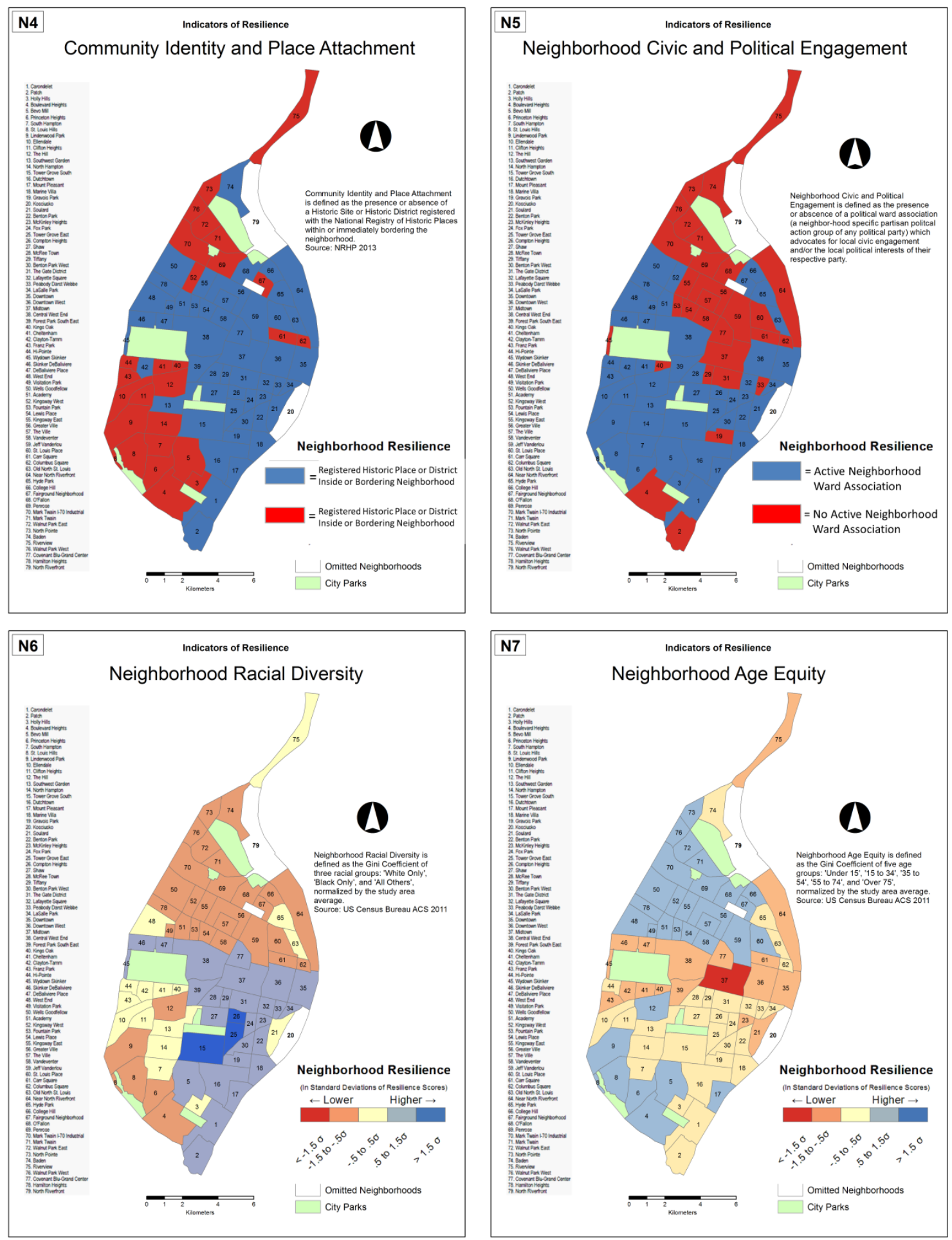

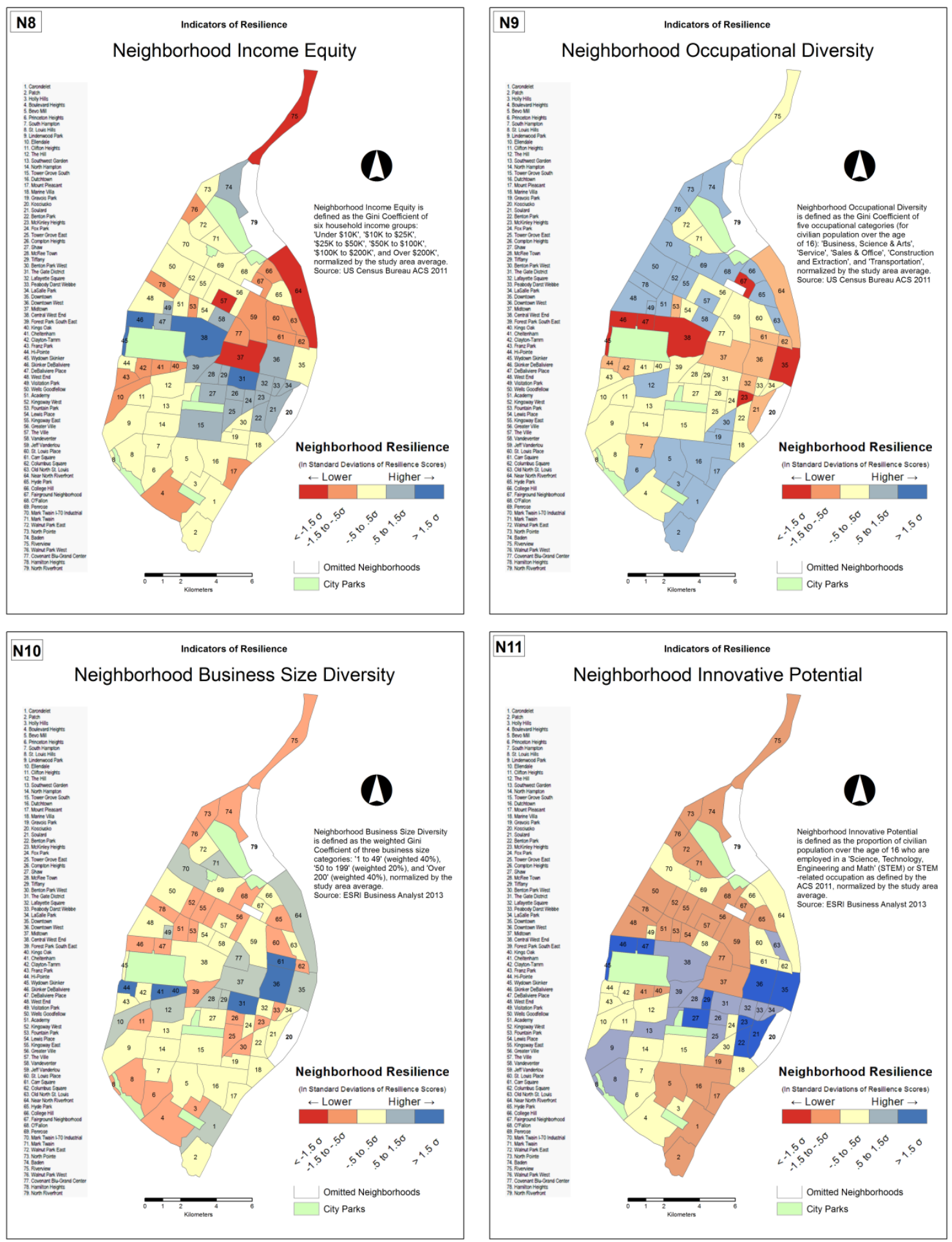

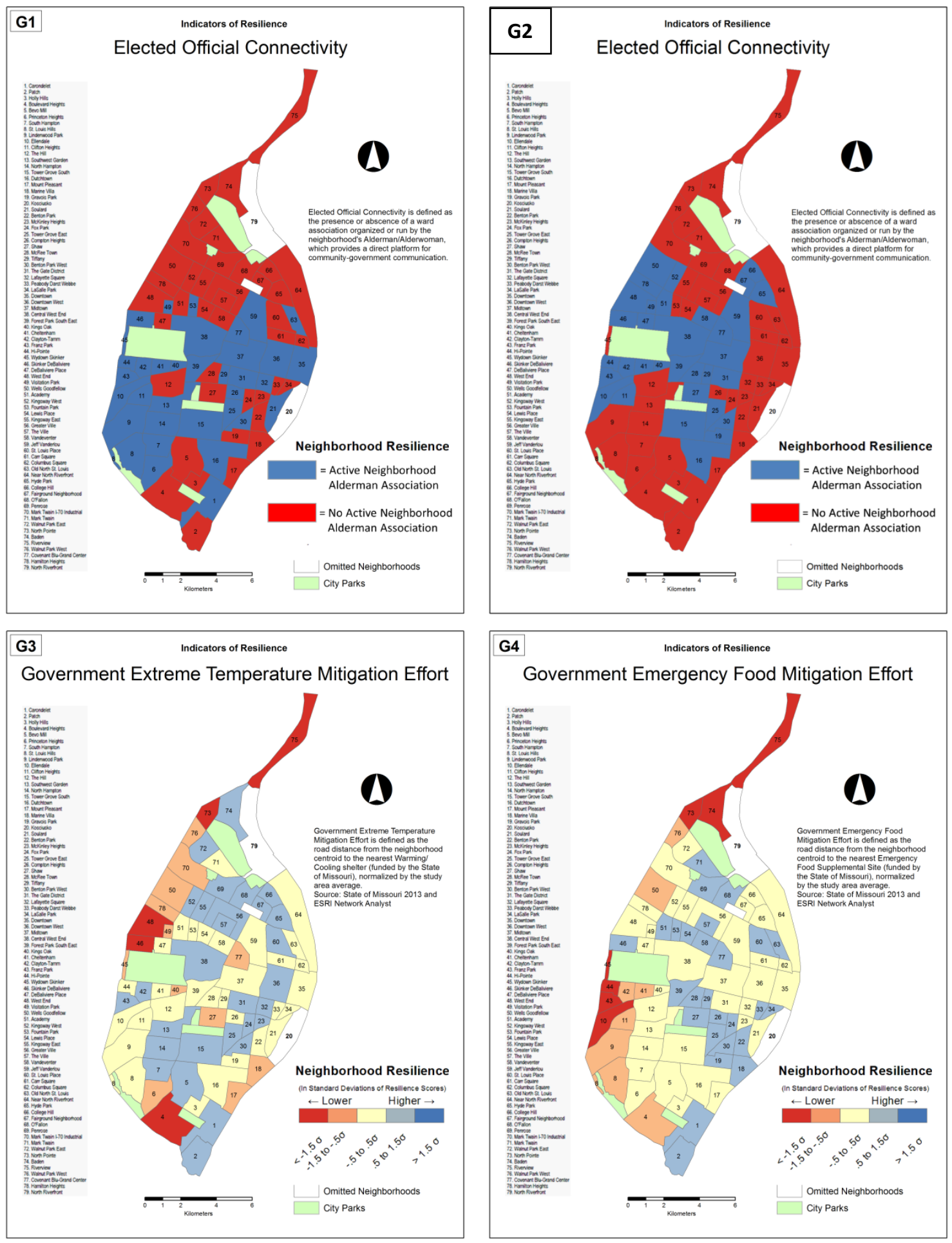

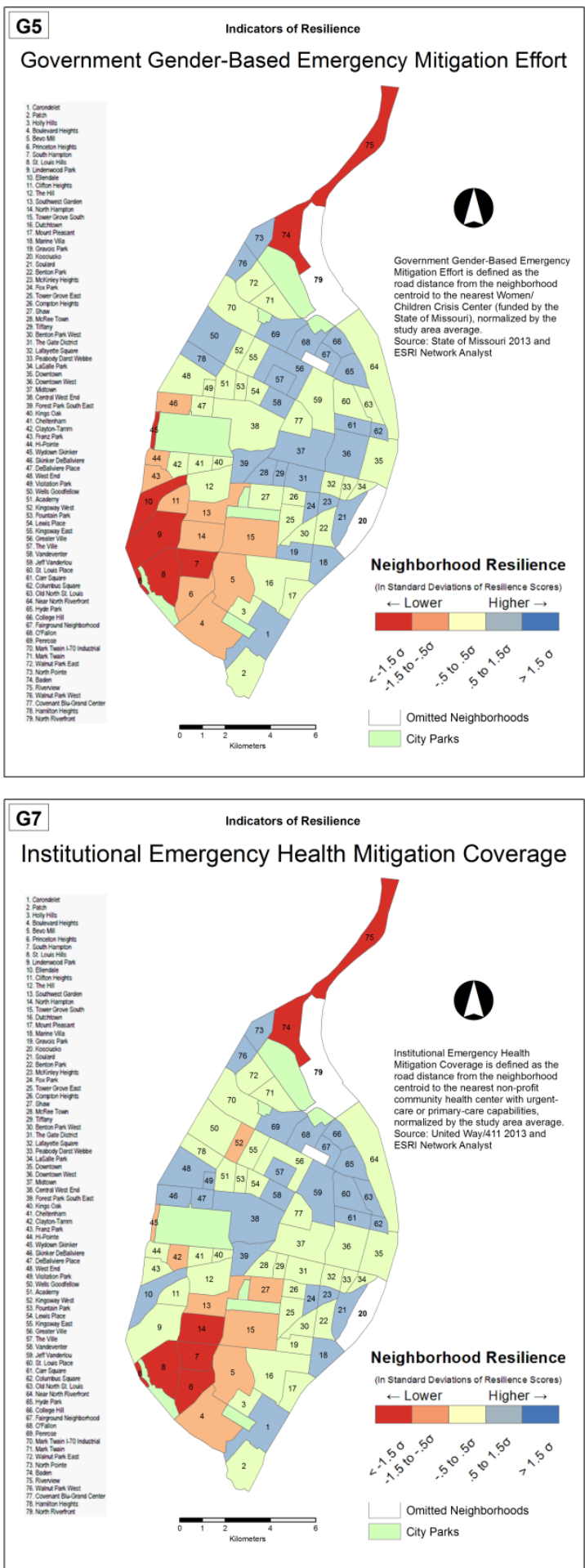

G6

Availability of Government-Maintained Open Space

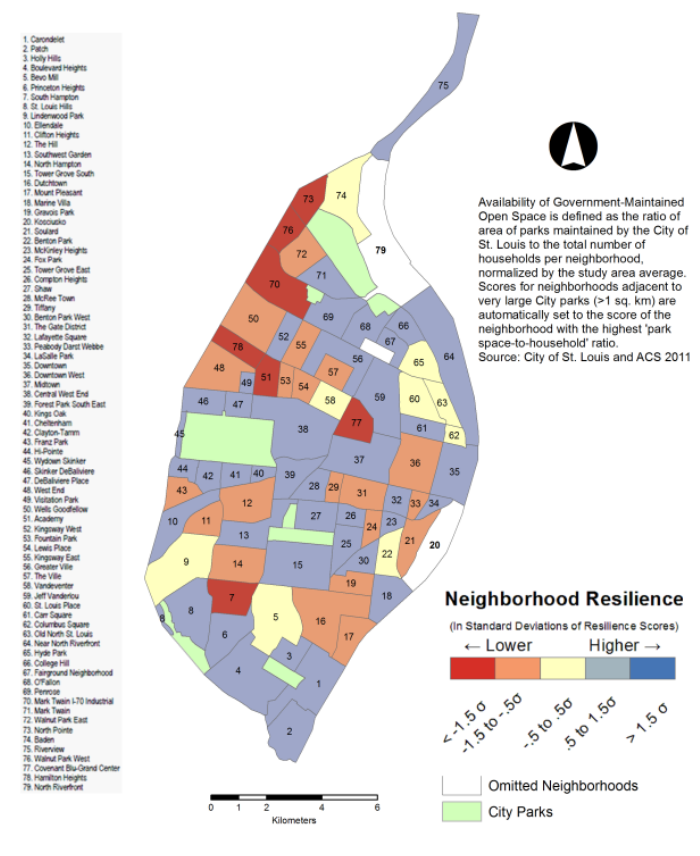

G8

Institutional Non-Profit / Community Services Presence
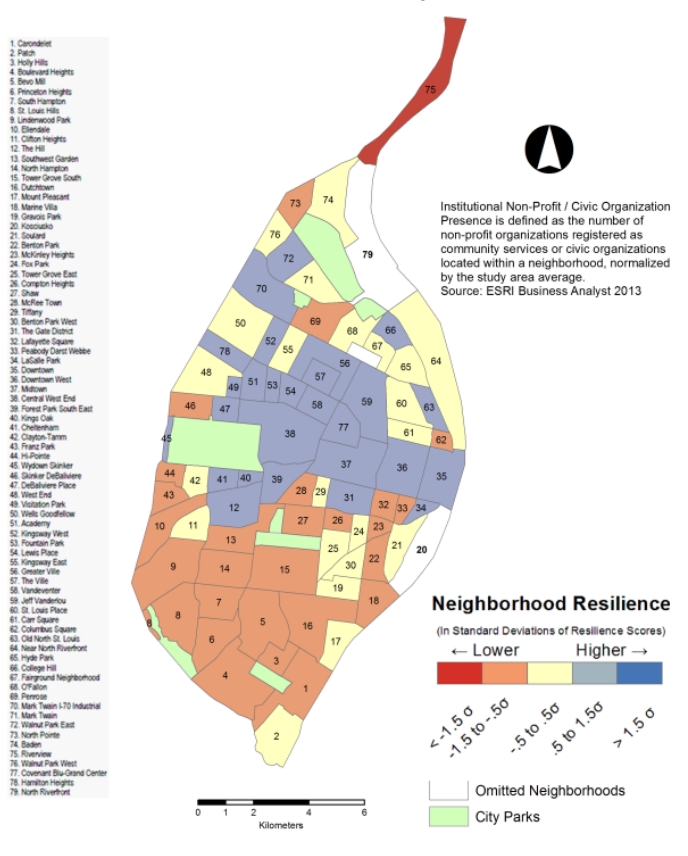


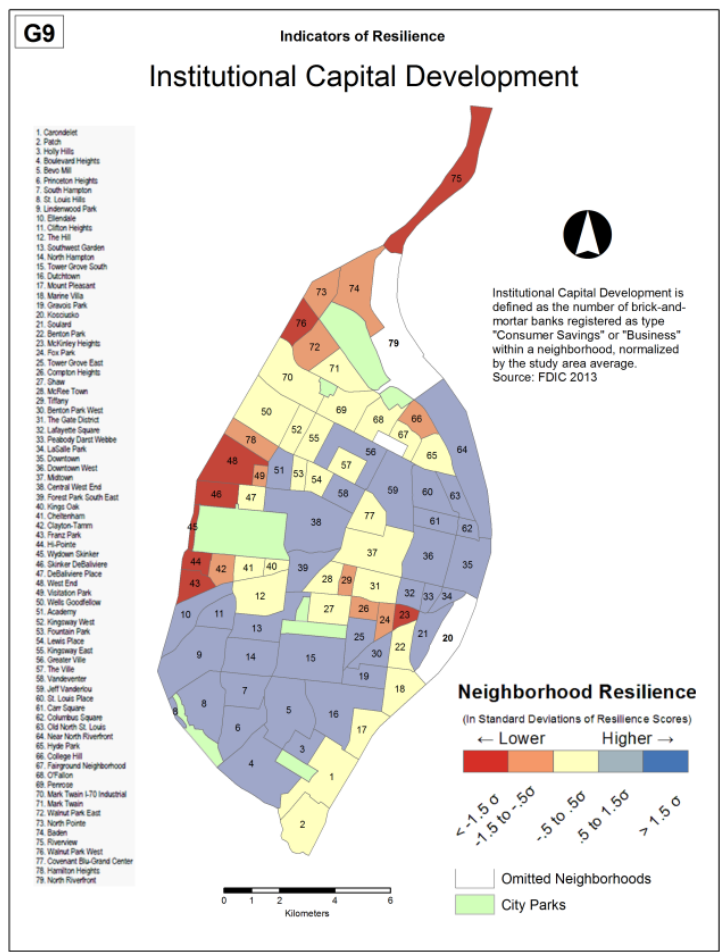




\section{BIBLIOGRAPHY}

Abdi H. and Williams L. (2010) 'Principal component analysis' Wiley Interdisciplinary Reviews: Computational Statistics vol 2 no 4 pp.433-459

Aczél J. and Saaty, T. L. (1983) 'Procedures for synthesizing ratio judgements' Journal of Mathematical Psychology vol 27 no 1 pp.93-102

Adger W., Brooks N., Bentham G., Agnew M. and Eriksen S. (2004) New indicators of vulnerability and adaptive capacity Tyndall Centre for Climate Change Research, Norwich, UK

Adrienko G. (2012) 'Identifying place histories from activity traces with an eye to parameter impact' Transactions on Visualization and Computer Graphics vol 18 no 5 pp.675-688

Alexander L. (1995) 'A survey of the field of natural hazards and disaster studies' in Carrerra A. and Guzzetti F. (Eds.) Geographical Information Systems in Assessing Natural Hazards Kluwer Academic Publishers, Boston pp.1-19

Alexander L. (1997) 'The study of natural disasters 1977-1997: Some reflections on a changing field of knowledge' Disasters vol 21 pp.284-304

Allen J. (2003) Lost Geographies of Power. Wiley-Blackwell, Oxford

Auf der Heide E. and Scanlon J. (2007) "Health and medical preparedness and response" In Emergency Management: Principles and Practice for Local Government W.L. Waugh and K. Tierney (eds.) Washington D.C.: International City Managers Association, pp.183-206

Bahadur A., Ibrahim M. and Tanner T. (2013) 'Characterising resilience: unpacking the concept for tackling climate change and development' Climate and Development vol 5 no 1 pp.55-65

Barnett J., Lambert S. and Fry I. (2008) 'The hazards of indicators: Insights from the environmental vulnerability index' Annals of the Association of American Geographers vol 98 no 1 pp.102-19

Barrows, H. (1923) 'Geography as human ecology' Annals of the Association of American Geographers vol 12 pp.1-14

Beams A. 'Situating natural hazards policy in urban political ecologies: New York 
neighborhoods and the aftermath of Hurricane Sandy' (Conference Paper) Association of American Geographers Annual Meeting 59, Tampa, Florida, $4 / 10 / 2104$

Bergren E. 'Mixed methods and hybrid epistemologies in climate change research' (Conference Paper) Association of American Geographers Annual Meeting 59, Tampa, Florida, 4/10/2104

Berkes F. and Ross H (2013) 'Community resilience: Toward an integrated approach' Society \& Natural Resources vol 26 no1 pp.5-20

Bhamra R., Dani S. and Burnard K (2011) 'Resilience: the concept, a literature review and future directions' International Journal of Production Research vol 49 no 18 pp.5375-5393

Bianchi S., Farley R. and Spain D. (1982) 'Racial inequalities in housing: An examination of recent trends' Demography vol 19 pp.37-51

Bitterman P. and Bennett D. (2014) 'Representing resilience in social-ecological systems: Trade-offs with sustainability and the effects of changing adaptive capacity' (Conference Paper) Association of American Geographers Annual Meeting 59, Tampa, Florida, 4/10/2104

Black R., Arnell N., Adger N., Thomas D., Geddes A. (2012) 'Migration, immobility and displacement outcomes following extreme events' Environmental Science and Policy no 27 pp.S32-S43

Blakie P., Cannon T., Davis I. and Wisner B. (1994) At risk: Natural Hazards, people's vulnerability and disasters Routledge, London

Bollman R., Beshiri R., and Mitura, V. (2006) Northern Ontario's communities: Economic diversification, specialization and growth Working Paper No. 82, Minister of Industry, Statistics Canada, Agriculture Division

Boon H., Cottrell A., King D., Stevenson R. and Millar, J. (2012) 'Bronfenbrenner's bioecological theory for modelling community resilience to natural disasters' Natural Hazards vol 60 no2 pp.381-408.

Boyden J. and Cooper E. (2007) 'Questioning the power of resilience: Are children up to the task of disrupting the transmission of poverty?' CPRC Working Paper 73, Chronic Poverty Research Centre, Oxford

Brand F. and Jax K. (2007) 'Focusing the meaning(s) of resilience: Resilience as a descriptive concept and a boundary object' Ecol. Soc. Vol 12 no 1 p.23 
Brinn M. and Greaves M. (2003) 'Leveraging agent properties to assure survivability of distributed multi-agent systems' In Proceedings of the second international joint conference on autonomous agents and multiagent systems (ACM) pp.946-947

Cardona O. (2005) Indicators of disaster risk and risk management: Summary report. Washington, DC: Inter-American Development Bank

Carlson J. and Doyle J. (2002) 'Complexity and robustness' Proceedings of the National Academy of Sciences of the United States of America vol 99 (Suppl 1) pp.25382545

Carpenter S., Walker B., Anderies J. M., and Abel N. (2001) 'From metaphor to measurement: resilience of what to what?' Ecosystems vol 4 no 8 pp.765-781

Chan J., DuBois B. and Tidball K. (2014) 'Places and spaces of resilience: Community gardens in post-Sandy New York' (Conference Paper) Association of American Geographers Annual Meeting 59, Tampa, Florida, 4/10/2104

Chan N. (1995) 'Choice and constraints in floodplain occupation: the influence of structural factors on residential location in Peninsular Malaysia' Disasters vol 19 no 4 pp.287-307

Chan N. and Wong H. (2007) 'Data mining of resilience indicators' IIE Transactions vol 39 no 6 pp.617-627

Chandra A., Williams M., Plough A., Stayton A., Wells K., Horta M. and Tang J. (2013) 'Getting actionable about community resilience: The Los Angeles County Community Disaster Resilience Project' American Journal of Public Health vol 103 no 7 pp.1181-1189

Clark J. (2006) 'The institutional limits to multifunctional agriculture: subnational governance and regional systems of innovation' Environment and Planning C: Government and Policy vol 24 pp.331-349

Cohen O., Leykin D., Lahad M., Goldberg A. and Aharonson-Daniel L. (2013) 'The conjoint community resiliency assessment measure as a baseline for profiling and predicting community resilience for emergencies' Technological Forecasting and Social Change vol 80 no 9 pp.1732-1741

Cont R. and Bouchaud, J. P. (2000) 'Herd behavior and aggregate fluctuations in financial markets' Macroeconomic Dynamics vol 4 no 2 pp.170-196

Cova T., and Church R. (1997) 'Modelling community evacuation vulnerability using GIS' International Journal of Geographical Information Science vol 11 no 8 pp.763-784 
Cumming G. Cumming H. and Redman C. (2006) 'Scale mismatches in social-ecological systems: causes, consequences, and solutions' Ecology and Society vol 11 no 1 p.14

Cutter S.L. and Solecki W. (1989) 'The national pattern of airborne toxic releases' The Professional Geographer vol 41 no 2 pp.149-161

Cutter S.L. (1993) Living with risk Routledge Chaplan and Hall, NY

Cutter S.L. (1996) 'Vulnerability to environmental hazards' Progress in Human Geography vol 20 no 4 pp.529-539

Cutter S.L., Mitchell J. and Scott M. (2000) 'Revealing the vulnerability of people and places: A case study of Georgetown County, South Carolina' Annals of the Association of American Geographers vol 90 no 4 pp.713-737

Cutter S.L. (2003) 'The vulnerability of science and the science of vulnerability' Annals of the Association of American Geographers vol 93 no 1 pp.1-12

Cutter S.L., Boruff B., Shirley W., (2003) 'Social vulnerability to environmental hazards.' Social Science Quarterly vol 84 no 1 pp.242-261

Cutter S.L., Barnes L., Berry M., Burton C., Evans E., Tate E. and Webb J. (2008) 'A placebased model for understanding community resilience to natural disasters' Global Environmental Change vol 18 no 4 pp.598-606

Cutter S.L., Burton C. and Emrich C. (2010) "Disaster resilience indicators for benchmarking baseline conditions," Journal of Homeland Security and Emergency Management vol 7 no 1

Davis, W.M. (1934) 'The Long Beach Earthquake' Geographical Review vol 24 no 1 pp.111

Dorfman R. (1979) 'A formula for the Gini coefficient' The Review of Economics and Statistics vol 61 pp.146-149

Downes B., Miller F., Barnett J., Glaister A. and Ellemor H. (2013) 'How do we know about resilience? An analysis of empirical research on resilience, and implications for interdisciplinary praxis' Environmental Resilience Letters vol 8 pp.2-9

Drabek T. (1986) Human system response to disaster: An inventory of sociological findings Springer-Verlag, NY

Durkheim E. (1951) Suicide Free Press, Glencoe, IL 
Dynes, R. (1970) Organized Behavior in Disaster D.C. \& Heath, Lexington, MA

East-West Gateway Council of Governments (EW Gateway2010a) St. Louis City Hazard Mitigation Plan (Part 1), E-W Gateway Council of Governments http://www.ewgateway.org/pdffiles/Library/HazMit/FinalDoc2010/AllHazMitPlan-Voll-STLCity.pdf

East-West Gateway Council of Governments (EW Gateway2010b) St. Louis City Hazard Mitigation Plan (Introduction), E-W Gateway Council of Governments http://www.ewgateway.org/pdffiles/Library/HazMit/FinalDoc2010/AllHazMitPlan-Voll-Intro.pdf

Ebert A., Kerle N. and Stein A. (2009) 'Urban social vulnerability assessment with physical proxies and spatial metrics derived from air-and spaceborne imagery and GIS data' Natural Hazards vol 48 no 2 pp.275-294

Eckert S., Jelinek R., Zeug G., Krausmann E. (2012) 'Remote sensing-based sssessment of tsunami vulnerability and risk in Alexandria, Egypt' Applied Geography vol 32 pp.714-723

Engie K. and Quiroga D. (2014) 'Searching for politics within complex adaptive systems' (Conference Paper) Association of American Geographers Annual Meeting 59, Tampa, Florida, 4/10/2104

Ferenczi S. (1921) Psycho-analysis and the war neuroses International Psychoanalytical Press

Flanagan E., Gregory E., Hallisey E., Heitgerd J. and Lewis B. (2011) "A Social Vulnerability Index for Disaster Management" Journal of Homeland Security and Emergency Management vol 8 no 1 Article 3

Flax L., Jackson R. and Stein D. (2002) 'Community vulnerability assessment tool methodology' Natural Hazards Review vol 3 no 4 pp.163-76

Fontela E, Gabus A (1976) The DEMATEL observer. Tech. rep. Battelle Geneva Research Center, Geneva

Friedman D. (1975) Computer simulation in natural hazard assessment (No. 2). Boulder, CO: Institute of Behavioral Science, University of Colorado

Garmestani A., Allen C., Mittelstaedt J., Stow C. and Ward W. (2006) 'Firm size diversity, functional richness, and resilience' Environment and Development Economics, vol 11 pp.533-551 
Garmezy N. (1991) 'Resilience and vulnerability to adverse developmental outcomes associated with poverty' American Behavioral Scientist, vol 34 pp.416-430

Geis D. (2000) 'By design: the disaster resistant and quality-of-life community' Natural Hazards Review vol 1 no 3 pp.151-160

Godschalk D. R. (2003) 'Urban hazard mitigation: Creating resilient cities' Natural Hazards Review, vol 4 no 3 pp.136-143

Goh K., Oh E., Jeong H., Kahng B. and Kim D. (2002). 'Classification of scale-free networks' Proceedings of the National Academy of Sciences vol 99 no 20 pp.12583-12588

Gunderson, L. (2000) 'Ecological resilience-in theory and application' Annual Review of Ecology and Systematics vol 31 no 1 pp.425-439

Gunderson L. (2010) 'Ecological and human community resilience in response to natural disasters' Ecology and Society vol 15 no2 p.18

Gunderson L. and Holling C., eds. (2002) Panarchy: Understanding Transformations in Human and Natural Systems Washington, DC: Island Press

Hall S. and Zautra A. (2010) 'Indicators of community resilience: What are they why bother?' in Reich J., Zautra A. and Hall J. (Eds.) Handbook of adult resilience. Guilford Press pp.350-371

Harkness S. (2007) 'Social and political indicators of human well-being' In M. McGillivray (Ed.), Human well-being: Concept and measurement New York: Palgrave MacMillan pp. 88-112

Heinz Center for Science, Economics, and the Environment (2002) Human Links to Coastal Disasters. Washington, D.C.: H. John Heinz Center.

Hiete M., Merz M., Comes T. and Schultmann F. (2012). 'Trapezoidal fuzzy DEMATEL method to analyze and correct for relations between variables in a composite indicator for disaster resilience' OR spectrum, vol 34 no 4 pp.971-995

Hewitt K. and Burton I. (1971) The hazardousness of place: A regional ecology of damaging events University of Toronto Department of Geography Research Publication no 6

Hewitt, K. (1983) 'The idea of calamity in a technocratic age' In K. Hewitt (Ed.), Interpretation of calamities pp.3-32 
Hoeflehner T. (2014) 'Integrated multilevel analysis of regional resilience - a transdisciplinary case study in southeast Austria' (Conference Paper) Association of American Geographers Annual Meeting 59, Tampa, Florida, 4/10/2104

Holling C.S. (1973) 'Resilience and stability of ecological systems' Annual Review of Ecological Systems vol 4 pp.1-23

Holling C., Gunderson L. and Peterson G. (2002) 'Sustainability and panarchies' in Panarchy: Understanding transformations in human and natural systems, Island Press pp.63-102

Houghton A., Prudent N., Scott J., Wade R., Luber G. (2012) ‘Climate change-related vulnerabilities and local environmental public health tracking through GEMSS: A web-based visualization tool' Applied Geography vol 33 pp.34-38

Howard S., Dryden J. and Johnson B. (1999) 'Childhood resilience: review and critique of literature' Oxford Review of Education vol 25 pp.307-323

Intergovernmental Panel on Climate Change (2013), 'Climate change in 2013: The physical science basis - Summary for policymakers' IPCC/WMO http://www.climatechange2013.org/images/uploads/WGI AR5 SPM brochure. pdf

Jabeen H., Johnson C. and Allen A. (2010) 'Built-in resilience: learning from grassroots coping strategies for climate variability' Environment and Urbanization vol 22 p.415

James L. and Hall B. (1986) 'Risk information for floodplain management' Journal of Water Resources Planning and Management vol 112 no 4 pp.485-499

James W. (1977) A pluralistic universe Harvard University Press, Cambridge MA

Jenks G. and Caspall F. (1971) 'Error on choroplethic maps: definition, measurement, reduction' Annals of the Association of American Geographers vol 61 no 2 pp.217-244

Johnson J. and Wielchelt S. (2004) 'Introduction to the special issue on resilience' Substance Use \& Misuse vol 39 no 5 pp.657-670

Jones B. and Andrey J. (2007) 'Vulnerability index construction: Methodological choices and their influence on identifying vulnerable neighborhoods' International Journal of Emergency Management vol 4 no 2 pp.269-95 
Kendra J. and Wachtendorf T. (2007). 'Community innovation and disasters' In Handbook of Disaster Research Springer: New York pp.316-334

King D. 2001. 'Uses and limitations of socio-economic indicators of community vulnerability to natural hazards: Data and disasters in Northern Australia' Natural Hazards vol 24 pp.147-56

Kit O., Lüdeke M. and Reckien D. (2012) 'Texture-based Identification of Urban Slums in Hyderabad, India Using Remote Sensing Data' Applied Geography vol 32 pp.660667

Krieger N. (2006) 'A century of census tracts: Health \& body politic (1906-2006)' Journal of Urban Health vol 83 no 3 pp.355-361

Kulig J. (2000) 'Community resiliency: The potential for community health nursing theory development' Public Health Nursing vol 17 no 5 pp.374-385

Kumari A. and Frazier T. (2014) 'Evaluating reliance on social capital for resilience: A case study of Hillsborough County, Florida' (Conference Paper) Association of American Geographers Annual Meeting 59, Tampa, Florida, 4/10/2104

Kutak R. (1938) 'The Sociology of Crisis: The Louisville Flood of 1938' Social Forces vol 17 pp.66-72

Larsen-Freeman D. and Cameron L. (2008) Complex systems and applied linguistics Oxford University Press

Lazarus, N. (2014) 'Evaluating community resilience under conditions of an environmental disaster: The case of the Deep Water Horizon oil spill' (Conference Paper) Association of American Geographers Annual Meeting 59, Tampa, Florida, 4/10/2104

LeichenkoR and Solecki W. (2014) 'Hurricane Sandy, disaster recovery, and the seeds of urban transformation' (Conference Paper) Association of American Geographers Annual Meeting 59, Tampa, Florida, 4/10/2104

Lew, A. (2014) Understanding and applying fast and slow resilience for rural tourism (Conference Paper) Association of American Geographers Annual Meeting 59, Tampa, Florida, 4/10/2104

Longstaff P., Armstrong N., Perrin K., Parker W. and Hidek M. (2010) 'Building resilient communities: A preliminary framework for assessment' Homeland Security Affairs vol 7 no 3 
Luthar S. and Zelazo L. B. (2003) 'Research on resilience: an integrative review', in S. Luthar Resilience and Vulnerability: Adaptation in the Context of Childhood Adversities, Cambridge/New York: Cambridge University Press, pp. 510-549

Manyena S. (2006) 'The concept of resilience revisited' Disasters vol 30 pp.433-50 Marston S. (1983) 'Natural Hazards Research: Toward a Political Economy Perspective' Political Geography Quarterly vol 2 no 4 pp.339-348

Matisziw T. C., Grubesic T. H. and Wei, H. (2008) 'Downscaling spatial structure for the analysis of epidemiological data' Computers, Environment and Urban Systems, vol 32 no 1, pp.81-93

Matisziw T. C., Grubesic T. H. and Guo J. (2012) 'Robustness elasticity in complex networks' Plos one, vol 7 no 7 e39788

McGranahan D., and Wojan T. (2007) 'Recasting the creative class to examine growth processes in rural and urban counties' Regional Studies vol 41 pp.197-216

McGuire L., Ford E. and Okoro C. (2007) 'Natural disasters and older US adults with disabilities: Implications for evacuation' Disasters vol 31 no 1 pp.49-56

Mileti D., Drabek T. and Haus E. (1977) 'Human systems in extreme Environments: A sociological perspective' Social Forces vol 55 no 4 pp.1093-1094

Mileti D. (1999) Disasters by Design: A Reassessment of Natural Hazards in the United States, Natural Hazards and Disasters. Washington, D.C.: Joseph Henry Press

Miller T., Baird C., Littlefield G., Kofinas F., Chapin I., and Redman C. (2008) 'Epistemological pluralism: reorganizing interdisciplinary research' Ecology and Society vol 13 no2 p.46

Mitchell J., Devine N. and Jagger K. (1989) 'A contextual model of natural hazard' Geographical Review vol 79 no 4 pp.391-409

Mohaupt S. (2009) 'Review article: Resilience and social exclusion' Social Policy and Society vol 8 no 1 pp.63-71

Montz B. (2000) 'The hazardousness of place: Risk from multiple natural hazards' Papers and Proceedings of the Applied Geography Conferences vol 23 pp.331-339

Montz B. and Tobin G. (2004) 'Natural hazards and technology: Vulnerability, risk and community response in hazardous environments' In S. D. Brunn, S. L. Cutter, J. W. Harrington (Eds.), Technoearth: A Social History of Geography and 
Technology Dordrecht, The Netherlands: Kluwer Academic Publishers pp. 547570

Montz B. and Tobin G. (2011) 'Natural hazards: An evolving tradition in applied geography' Applied Geography vol 31 pp.1-4

Morgan R., and Brown J (1996) 'Using giving-up densities to detect search images.' American Naturalist pp.1059-1074

Morrow B. (1999) 'Identifying and mapping community vulnerability' Disasters vol 23 no 1 pp.1-18

Morrow B. (2008) Community resilience: A social justice perspective (Vol. 4). Oak Ridge, TN: CARRI Research Report

Mustafa D. (2011) 'Pinning down vulnerability: From narratives to numbers' Disasters vol 35 no 1 pp.62-86

National Association of Charter School Authorizers (NACSA 2009) Public Schools in St.Louis: Place, Performance, Promise

National Research Council (2000) 'Ecological indicators for the nation' Washington D.C.: National Academy Press

National Research Council (2006) 'Facing hazards and disasters: Understanding human dimensions' Washington, DC: National Academy Press

National Research Council (2012) Steinbruner J., Stern P., Husbands J. (Eds.) 'Climate and social stress: Implications for national security' Washington D.C.: National Academies Press

Norris F., Stevens S., Pfefferbaum B., Wyche K. and Pfefferbaum R. (2008) 'Community resilience as a metaphor, theory, set of capacities, and strategy for disaster readiness.' American Journal of Community Psychology vol 41 pp.127-150

Obrist B., Pfeiffer C., and Henley R. (2010). 'Multi-layered social resilience: A new approach in mitigation research' Progress in Development Studies vol 10 pp.283293

O'Keefe P., Westgate K., and Wisner B. (1976) 'Taking the naturalness out of natural disasters' Nature vol 260 pp.566-567

Palm, R. (1990) Natural hazards: An integrative framework for research and planning John Hopkins University Press, Baltimore 
Parris T. and Kates R. (2003) 'Characterizing and measuring sustainable development' Annual Review of Environmental Resource Management vol 28 no 1 pp.559-586

Paul B. K. (2011) Environmental hazards and disasters: Contexts, perspectives and management Wiley-Blackwell, West Sussex UK

Pendall R., Foster K. and \& Cowell M. (2010) 'Resilience and regions: building understanding of the metaphor' Cambridge Journal of Regions, Economy and Society vol 3 no 1, pp.71-84

Prince S. (1925) Catastrophe and Social Change Columbia University Press, NY

Quarantelli E. and Dynes R. (1977) 'Response to Social Crisis and Disaster' Annual Review of Sociology vol 3 pp.23-49

E. L. Quarantelli (1979) 'STUDIES IN DISASTER RESPONSE AND PLANNING' University of Delaware Disaster Research Center FINAL PROJECT REPORT \#24 January 1979

Ratick S. and Osleeb J. P. (2013) 'Measuring the vulnerability of populations susceptible to lead contamination in the Dominican Republic: evaluating composite index construction methods' GeoJournal vol 78 no 2 pp.259-272

Reghezza-Zitt M., Rufat S., Djament-Tran G., Blanc A. and Lhomme S. (2012) 'What Resilience Is Not: Uses and Abuses' Cybergeo: European Journal of Geography [En ligne] Environnement, Nature, Paysage, document \#621

Renschler C., Frazier A., Arendt L., Cimellaro G. P., Reinhorn A. M. and Bruneau, M. (2010) 'A framework for defining and measuring resilience at the community scale: The PEOPLES resilience framework' Proceedings of the 9th U.S. National and 10th Canadian Conference on Earthquake Engineering, 2010, Toronto, Ontario, Canada Paper No 1827

Richmond N and Sovacool B. (2012) 'Bolstering resilience in the Coconut Kingdom: Improving adaptive capacity to climate change in Vanuatu' Energy Policy vol 50 pp.843-858

Robinson W. (1950) 'Ecological correlations and the behavior of individuals' American Sociological Review vol 15 pp.351-57

Rodina L. (2014) Conceptualizing resilience from lived experience perspectives: the case of informal settlements in Cape Town, South Africa' (Conference Paper) Association of American Geographers Annual Meeting 59, Tampa, Florida, $4 / 10 / 2104$ 
Rufat S. (2013) 'Spectroscopy of urban vulnerability' Annals of the Association of American Geographers vol 103 no 3 pp.505-525

Rutter M. (2012) 'Resilience as a dynamic concept' Development and Psychopathology Cambridge University Press vol 24 pp.335-344

Saaty T. L. (2001) Decision making for leaders: The Analytic Hierarchy Process for decisions in a complex world 1999/2000 Edition (Vol. 2). RWS publications.

Saaty, T. L. (2008) 'Relative measurement and its generalization in decision making: why pairwise comparisons are central in mathematics for the measurement of intangible factors' RACSAM-Revista de la Real Academia de Ciencias Exactas, Fisicas y Naturales. Serie A. Matematicas vol 102 no 2 pp.251-318

Scholz C. (2002) The mechanics of earthquakes and faulting Cambridge University Press

Shaw-Taylor Y (1999) Measurement of community health: The social health index. New York: University Press of America, Inc.

Sherrieb K., Norris F., and Galea, S. (2010) 'Measuring capacities for community resilience' Social Indicators Research, vol 99 no 2 pp.227-247

Simon, H. (1956) 'Rational choice and the structure of the environment' Psychological Review vol 63 pp.129-138

Skinner D., Matthews S., and Burton L. (2005) 'Combining ethnography and GIS technology to examine constructions of developmental opportunities in contexts of poverty and disability' In T. S. Weisner (Ed.), Discovering successful pathways in children's development Chicago: The University of Chicago Press pp.223-239

Smith, K. (2001) Environmental hazards: Assessing risk and reducing disaster Routledge, London

Song W., Weicheng F., Binghong W. and Jianjun Z. (2001) 'Self-organized criticality of forest fire in China' Ecological Modelling vol 145 no 1 pp.61-68

St. Louis City Dept. of Planning and Urban Design (STL Dept. Planning 2010) City-Wide Neighborhood Map https://stlouis-mo.gov/government/departments/planning/ documents/upload/CitywideNeighborhoodMap_TabloidSzRev.pdf

St. Louis City Dept. of Public Health (STL Health Dept. 2012) Understanding Our Needs City of St. Louis Department of Public Health Center for Health Information, Research and Planning 
St. Louis Post-Dispatch (2012) 'Stubbornness Ban Be Lethal In St. Louis Heat Wave' July 23, 2012, Blythe Bernhard http://www.stltoday.com/news/local/metro/stubbornness-can-be-lethal-in-stlouis-heat-wave/article 404ef3ad-815f-5c90-9753-185fa87d2d27.html

St. Louis Post-Dispatch (2010) 'Storm, tornadoes leave damage scattered over St. Louis area' December 31, 2010, Staff

http://www.stltoday.com/news/local/metro/storm-tornadoes-leave-damagescattered-over-st-louis-area/article_33cc4f6c-14e4-11e0-8e19-

0017a4a78c22.html

Sterman J. (2000) Business dynamics: Systems thinking and modeling for a complex world Irwin-McGraw-Hill

Stewart G., Kolluru R. and Smith M. (2009) 'Leveraging public-private partnerships to improve community resilience in times of disaster' International Journal of Physical Distribution \& Logistics Management, vol 39 no 5 pp.343-364

Stollenwerk N. (2005) 'Criticality in epidemics: The mathematics of sandpiles explains uncertainty in epidemic outbreaks' In Recent Advances in Applied Probability Springer, pp.455-494

Subcommittee on Disaster Reduction (SDR). 2005 Grand challenges for disaster reduction National Science and Technology Council, Washington D.C.

Subramanian S., Jones K., Kaddour A.and Krieger N. (2009) 'Revisiting Robinson: the perils of individualistic and ecologic fallacy' International Journal of Epidemiology vol 38 no 2 pp.342-360

Susman P., O'Keefe P., and Wismer B. 'Global disasters: A radical interpretation' in Hewitt, K (Ed.) Interpretations of Calamity Allen \& Unwin, NY pp.263-283

Sylves R. (2007) 'Budgeting for local emergency management and homeland security' In Emergency Management: Principles and Practice for Local Government, W.L. Waugh and K. Tierney (eds.) Washington, D.C.: International City Managers Association, pp. 183-206.

Tamura H.and Akazawa K. (2005) 'Stochastic DEMATEL for structure modeling of a complex problematique for realizing safe, secure and reliable society' Journal of Telecommunication Information Technology vol 4 pp.139-146

Tate E. (2012) 'Social vulnerability indices: a comparative assessment using uncertainty and sensitivity analysis' Natural hazards vol 63 no 2 pp.325-347 
Tate E. (2013) 'Uncertainty analysis for a social vulnerability index' Annals of the Association of American Geographers no 103 vol 3 pp.526-543

Taylor A. (1984) 'Architecture and society: Disaster structures and human stress' Ekistics vol 51 pp.446-451

Teo M., Goonetilleke A, and Ziyath A. (2013) 'An integrated framework for assessing community resilience in disaster management' In Proceedings of the 9th Annual International Conference of the International Institute for Infrastructure Renewal and Reconstruction (Conference Paper) Queensland University of Technology, Brisbane, QLD

The Reinvestment Fund (2013) Searching For markets: The geography of inequitable access to healthy and affordable food in the United States. US Department of the Treasury CDFI Fund, Washington http://www.trfund.com/wpcontent/uploads/2013/07/SearchingForMarketsFullReport.pdf

Tidball K., and Krasny M. (2007) 'From risk to resilience: what role for community greening and civic ecology?' in Wals A., ed. Social learning towards a more sustainable world Wageningen Academic Publishers, Wageningen, The Netherlands pp.149-164

Tierney K. and Bruneau M. (2007) 'Conceptualizing and measuring resilience: A key to disaster loss reduction' TR News vol 250 pp.14-17

Torry W. (1979) 'Hazards, hazes and holes: A critique of The Environment as Hazard and general reflections on hazard research' Canadian Geographer vol 23 pp.368-83

Turner B. (2010) 'Vulnerability and resilience: coalescing or paralleling approaches for sustainability science? Global Environmental Change vol 20 pp.570-576

Ungar M. (2011) 'The social ecology of resilience: Addressing contextual and cultural ambiguity of a nascent construct' The American Journal of Orthopsychiatry vol 81 pp.1-17

Twigg J. (2007) 'Characteristics of a disaster-resilient community: a guidance note' DFID Disaster Risk Reduction Interagency Coordination Group

United Nations Development Programme (UNDP 1990) Human development report. New York: Oxford University Press

U.S. Census Bureau (2009a) Cartographic Boundary Files http://www.census.gov/geo/www/cob/tr metadata.html 
Vale L. and Campanella T. (2005) The resilient City: How modern cities recover from disaster New York: Oxford University Press

Veland S., Howitt R., Dominey-Howes D., Thomalla F. and Houston D. (2012) 'Procedural vulnerability: Understanding environmental change in a remote indigenous community' Global Environmental Change no 1044

Vogel C., Moserb S., Kasperson R., Dabelkod G. (2007) 'Linking vulnerability, adaptation, and resilience science to practice: Pathways, players, and partnerships' Global Environmental Change vol 17 pp.349-363

Waddell E. (1977) 'The Hazards of Scientism: A Review Article' Human Ecology vol 5 pp.69-76

Waddell E. (1983) 'Coping with frosts, governments and disaster experts: Some reflections based on a New Guinea experience and a perusal of the relevant literature' in Hewitt, K (Ed.) Interpretations of Calamity Allen \& Unwin, NY pp.3343

Wash-Diley M., Wolford, W. and McCarthy J. (2014) 'A political ecology of resilience? Stories from participatory community evaluations' (Conference Paper) Association of American Geographers Annual Meeting 59, Tampa, Florida, $4 / 10 / 2104$

Windle G. (2011) 'What is resilience? A review and concept analysis' Reviews in Clinical Gerontology vol 21 no 2 pp.152-169

White G. (1942) Human adjustment to floods: A geographical approach to the flood problem in America University of Chicago Press, Chicago

White G. and Haus J. (1975) Assessment of research on natural hazards MIT Press, Cambridge

White G., Burton I. and Kates R. (1978) The environment as hazard Guilford Press, NY

Wilson G. (2012) 'Community resilience, globalization, and transitional pathways of decision-making' Geoforum vol 43 pp.1218-1231

Wolf J., Adger W., Lorenzoni I., Abrahamson V. and Raine R. (2010) 'Social capital, individual responses to heat waves and climate change adaptation: An empirical study of two UK cities' Global Environmental Change vol 20 no 1 pp.44-52

Wolf T., McGregor G. and Analitis A. (2014) 'Performance assessment of a heat wave 
vulnerability index for greater London', United Kingdom. Weather, Climate \& Society, vol 6 no 1

Wu H., Shieh J., Lee Y. and Chen H. (2010) 'A combination of AHP and DEMATEL in evaluating the criteria of employment service outreach program personnel' Information Technology Journal vol 9 no 3 pp.569-575

Zahedi F. (1986) 'The analytic hierarchy process-a survey of the method and its applications' Interfaces vol 16 no 4 pp.96-108

Zadeh L. (1973) 'Outline of a new approach to the analysis of complex systems and decision processes' IEEE Transactions on Systems, Man and Cybernetics vol 1 pp.28-44 